\title{
ULTRASONIC PLY-BY-PLY DETECTION OF MATRIX CRACKS IN LAMINATED COMPOSITES
}

\author{
A Thesis \\ by \\ ATUL SHRIDATTA GANPATYE

\begin{abstract}
Submitted to the Office of Graduate Studies of
Texas A\&M University

in partial fulfillment of the requirements for the degree of

MASTER OF SCIENCE
\end{abstract}

December 2004

Major Subject: Aerospace Engineering 


\title{
ULTRASONIC PLY-BY-PLY DETECTION OF MATRIX CRACKS IN LAMINATED COMPOSITES
}

\author{
A Thesis \\ by \\ ATUL SHRIDATTA GANPATYE \\ Submitted to Texas A\&M University \\ in partial fulfillment of the requirements \\ for the degree of \\ MASTER OF SCIENCE
}

Approved as to style and content by:

\begin{tabular}{c}
\hline Vikram K. Kinra \\
(Chair of Committee) \\
\hline Ozden O. Ochoa \\
(Member)
\end{tabular}

Vikram K. Kinra

(Member)

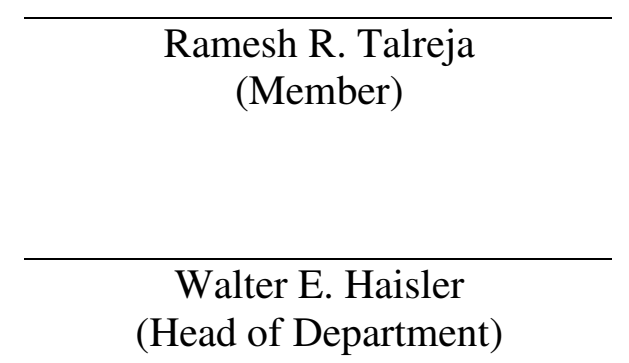

December 2004

Major Subject: Aerospace Engineering 


\author{
ABSTRACT \\ Ultrasonic Ply-by-ply Detection of Matrix Cracks \\ in Laminated Composites. (December 2004) \\ Atul Shridatta Ganpatye, B.E., Veermata Jeejabai Technological Institute \\ Chair of Advisory Committee: Dr. Vikram K. Kinra
}

In the design of cryogenic fuel tanks for the next generation Reusable Launch Vehicles (RLVs), the permeability of liquid hydrogen $\left(\mathrm{LH}_{2}\right)$ across the thickness of the tank is a critical issue. The rate of permeation of $\mathrm{LH}_{2}$ is largely dependent on the internal damage state of the composite tank wall. Damage in the form of matrix cracks in the composite material of the tank is responsible for the through-the-thickness permeation of $\mathrm{LH}_{2}$. In this context, the detection of matrix cracks takes on an unprecedented significance. In this work, an ultrasonic technique for the ply-by-ply detection of matrix cracks in laminated composites is developed. Experimental results are presented for graphite/epoxy laminates with different lay-ups and laminate thicknesses. Matrix cracks in each of the plies of the laminated composites were detected even when there was a rather high density of cracks in all of the plies. The ultrasonic data were calibrated by comparing them with the corresponding results obtained by using the traditional methods of optical microscopy and penetrant enhanced X-radiography. Excellent quantitative correlation was observed between the results obtained with ultrasonics and the traditional methods. 


\section{DEDICATION}

To my wife, Sheetal, and my parents 


\section{ACKNOWLEDGMENTS}

The author wishes to express endless gratitude to his advisor, Dr. Vikram K. Kinra, for his continual support and guidance throughout the entire course of his graduate studies at Texas A\&M University. Without his wise counsel and encouragement at every stage, this thesis would have remained but a dream.

The author is extremely grateful to Dr. Ramesh Talreja and Dr. Ozden Ochoa for taking time to serve on the committee and for providing valuable insights and additional perspectives that have helped to raise the quality of this document considerably.

The author extends his special appreciation to Dr. Konstantin Maslov for providing his time and willing assistance at various stages of this work. Special thanks are due to Dr. Kristofer Gamstedt (Royal Institute of Technology (KTH), Stockholm, Sweden), and to Dr. J. André Lavoie (Virginia Polytechnic Institute and State University, Blacksburg, Virginia) for their numerous thoughts on many topics pertinent to this research. The author is also grateful to Mr. Scotty Sparks (NASA Marshall Space Flight Center, Huntsville, Alabama) for many helpful discussions during the course of this investigation, and for supplying the specimens.

This work was supported by NASA through the National Center for Advanced Manufacturing - Louisiana Partnership, grant \# NCC8-223. Any opinions, findings, and conclusions or recommendations expressed in this material are those of the authors and do not necessarily reflect the views of the National Aeronautics and Space Administration. 


\section{TABLE OF CONTENTS}

Page

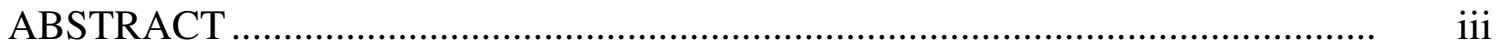

DEDICATION .................................................................................... iv

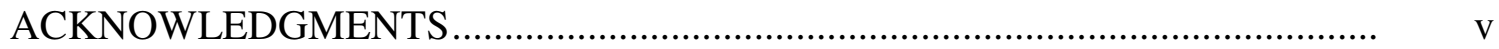

TABLE OF CONTENTS ......................................................................... vi

LIST OF FIGURES ............................................................................. viii

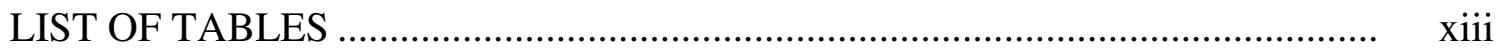

\section{CHAPTER}

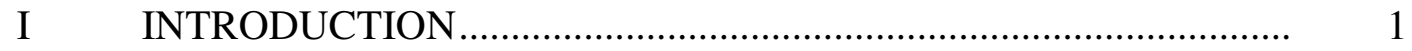

1.1 Motivation and Objective................................................. 1

1.2 Overview of the Techniques Used for the Detection of Matrix Cracks in Composites ............................................. 5

1.2.1 Optical Microscopy ................................................... 5

1.2.2 Penetrant Enhanced X-radiography .......................... 7

1.2.3 Ultrasonics.......................................................... 11

$1.3 \quad$ Scope of This Thesis ................................................... 17

II $\quad$ EXPERIMENTAL PROCEDURES …............................................ 19

2.1 Specimens and Materials................................................. 19

$2.2 \quad$ Optical Microscopy and X-radiography ................................ 26

$2.3 \quad$ Ultrasonic Test Equipment................................................. 27

$2.4 \quad$ Testing Procedures .............................................................. 36

2.4.1 Ultrasonic Polar Backscattering .............................. $\quad 36$

2.4.2 Calibration Techniques ........................................ 47

2.4.3 Data Corroboration...................................................... 55

III $\quad$ RESULTS AND DISCUSSION ................................................. 74

3.1 TAMU-1 Specimen ....................................................... 74

3.2 TAMU-2, TAMU-3 and TAMU-4 Specimens ..................... 84

3.3 TAMU-5 Specimen ............................................................ 94 
CHAPTER $\quad$ Page

IV CONCLUSIONS .................................................................... 101

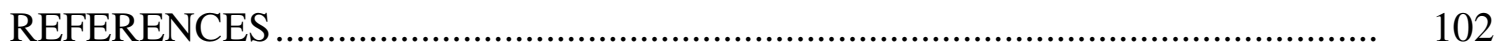

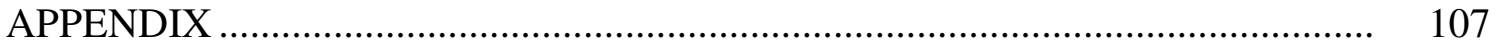

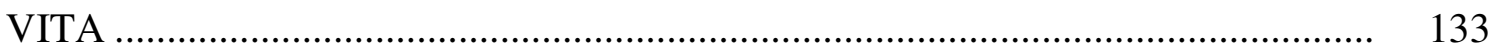




\section{LIST OF FIGURES}

Page

Fig. 1.1 Optical micrograph showing matrix cracks in the fourth and the fifth ply of an eight-ply [90/45/0/-45] graphite/epoxy laminate.

Fig. 1.2 A schematic of the possible arrangements of matrix cracks in a damaged [90/0/90] laminate

Fig. 1.3 A schematic illustrating (a) a through crack, (b) a partial crack and (c) an internal crack in a single ply

Fig. 1.4 A schematic showing the cracks in the first ply $\left(90^{\circ}\right)$ of a $[90 / 45 / 0 /-45]_{\mathrm{s}}$ graphite/epoxy laminate as detected by optical microscopy

Fig. 1.5 X-ray image showing matrix cracks (dark lines) in a an eight-ply $[90 / 45 / 0 /-45]_{\mathrm{s}}$ graphite/epoxy laminate

Fig. 1.6 A schematic of a typical ultrasonic nondestructive evaluation setup

Fig. 1.7 A schematic of setup for the detection of matrix cracks in a laminated composite using the normal incidence ultrasonic technique

Fig. 2.1 A photograph of the eight-ply TAMU-1 specimen

Fig. 2.2 A schematic showing the location of TAMU-2, TAMU-3 and TAMU-4 specimens on the corresponding composite discs

Fig. 2.3 Photographs of the TAMU-2, TAMU-3 and TAMU-4 specimens

Fig. 2.4 An optical micrograph of an edge of the TAMU-2 specimen showing the aluminum liner

Fig. 2.5 An optical micrograph of an edge of the TAMU-3 specimen showing the tedlar (polyvinyl fluoride) liner

Fig. 2.6 A schematic of the 18-ply TAMU-5 specimen.................................... 25

Fig. 2.7 A schematic representation of the ultrasonic scanning system ................. 28

Fig. 2.8 Response of the $20 \mathrm{MHz}$ transducer; (a) time domain, (b) frequency domain 
Fig. 2.9 Response of the $25 \mathrm{MHz}$ transducer; (a) time domain,

(b) frequency domain

Fig. 2.10 Characteristics of the acoustic beam of a focused transducer

Fig. 2.11 A schematic of the concept underlying the ultrasonic polar backscattering technique

Fig. 2.12 A schematic showing a general spatial arrangement of the ultrasonic transducer with respect to a cracked ply or ply-group

Fig. 2.13 Variation in the amplitude of the reflected signal with changes in the azimuthal angle $(\beta)$

Fig. 2.14 A schematic of the spatial arrangement of the ultrasonic transducer with respect to a cracked ply or ply group

Fig. 2.15 Variation in the amplitude of the reflected signal with changes in the angle of incidence $(\alpha)$

Fig. 2.16 Variation in the amplitude of the reflected signal with changes in the vertical distance between the transducer and the specimen

Fig. 2.17 Typical time domain signals observed in case of the TAMU-5 specimen using the polar backscattering technique

Fig. 2.18 A schematic of the experimental setup for the ultrasonic pitch-catch method.

Fig. 2.19 Typical time domain signals observed in case of the CALB-1 specimen using the pitch-catch technique shown in Fig. 2.16.

Fig. 2.20 Ultrasonic images of matrix cracks in the $\left[0 / 90_{4}\right]_{\mathrm{s}}$ CALB-1 laminate

Fig. 2.21 Ultrasonic images of matrix cracks in the $\left[0 / 90_{3}\right]_{\mathrm{s}}$ CALB-2 laminate

Fig. 2.22 A schematic showing the arrangement of the transducers $A$ and $B$ in order that the signal from transducer $A$ is reflected towards transducer B

Fig. 2.23 Image of cracks in the $90_{8}$ ply-group of the 10-ply CALB-1 specimen ...... 
Fig. 2.24 Image of cracks in the $90_{6}$ ply-group of the eight-ply

CALB-2 specimen

Fig. 2.25 Comparison between the images of the cracks in the $90_{8}$ ply-group of the CALB-1 laminate as obtained by different techniques

Fig. 2.26 Comparison between the images of the cracks in the $90_{6}$ ply-group of the CALB-2 laminate as obtained by different techniques

Fig. 2.27 An X-ray image of a part of the eight-ply TAMU-1 specimen showing matrix cracks (dark lines)

Fig. 2.28 A comparison of a part of the X-ray image of the TAMU-1 laminate with the schematic of the optically detected matrix cracks

Fig. 2.29 A comparison of a part of the X-ray image of the TAMU-2 laminate with the schematic of the optically detected matrix cracks at the edges

Fig. 2.30 A comparison of a part of the X-ray image of the TAMU-3 laminate with the schematic of the optically detected matrix cracks at the edges

Fig. 2.31 A comparison of a part of the X-ray image of the TAMU-4 laminate with the schematic of the optically detected matrix cracks at the edges

Fig. 2.32 X-ray image of a part of the TAMU-5 laminate 68

Fig. 2.33 (a) Filtered X-ray image, and (b) ultrasonic image, of cracks in the $-45_{2}$ ply-group of the TAMU-1 specimen.

Fig. 2.34 (a) Filtered X-ray image, and (b) ultrasonic image, of cracks in the $-45_{2}$ ply-group of the TAMU-2 specimen

Fig. 2.35 (a) Filtered X-ray image, and (b) ultrasonic image, of cracks in the $-45_{2}$ ply-group of the TAMU-3 specimen.

Fig. 2.36 (a) Filtered X-ray image, and (b) ultrasonic image, of cracks in the $-45_{2}$ ply-group of the TAMU-4 specimen. 
Fig. 3.1 Ultrasonic images of cracks in the eight plies of the [90/45/0/-45] $]_{\mathrm{s}}$ TAMU-1 specimen.

Fig. 3.2 Typical time domain signals; (a) in the absence of crack, (b) when a crack is insonified by the incident acoustic beam

Fig. 3.3 (a) Short cracks in second ply $\left(45^{\circ}\right)$ of the TAMU-1 specimen, driven by cracks in the first ply $\left(90^{\circ}\right)$, (b) corresponding driving cracks in the first ply, (c) schematic of the optically detected cracks in the first ply

Fig. 3.4 (a) Cracks in first ply $\left(90^{\circ}\right)$, (b) short cracks in the corresponding area in the second ply $\left(45^{\circ}\right)$, (c) enlarged views of the areas marked $A$ and $C,(d)$ enlarged views of the areas marked B and D

Fig. 3.5 Typical time-domain signal for the TAMU-4 specimen when the incident ultrasonic beam insonifies a crack through a rough surface

Fig. 3.6 Effect of gate positioning on image of cracks in the first ply of the TAMU-4 specimen

Fig. 3.7 Effect of gate positioning on image of cracks in the first ply (ply adjacent to the aluminum liner) of the TAMU-2 specimen

Fig. 3.8 Ultrasonic images of cracks in the eight plies of the $[90 / 45 / 0 /-45]_{\mathrm{s}}$ TAMU-2 specimen

Fig. 3.9 Ultrasonic images of cracks in the eight plies of the [90/45/0/-45] $]_{\mathrm{s}}$ TAMU-3 specimen.

Fig. 3.10 Ultrasonic images of cracks in the eight plies of the $[90 / 45 / 0 /-45]_{\mathrm{s}}$ TAMU-4 specimen.

Fig. 3.11 (a) X-ray image showing short cracks in the second ply $\left(45^{\circ}\right)$ of the TAMU-2 specimen, (b) comparison of the results of serial sectioning with the $\mathrm{x}$-ray image, and (c) schematic of the optically detected edge crack positions in the second ply

Fig. 3.12 Ultrasonic images of matrix cracks in the TAMU-5 specimen 
Fig. 3.13 Ultrasonic image of matrix cracks in the third ply of the TAMU-5 specimen obtained by using two different acoustic paths

Fig. 3.14 Ultrasonic image of matrix cracks in the fourth ply of the TAMU-5 specimen obtained by using two different acoustic paths

Fig. 3.15 A photograph of the TAMU-5 specimen showing the strain gage ............ 100 


\section{LIST OF TABLES}

Page

Table $1 \quad$ List of Calibration Specimens ...................................................... 20

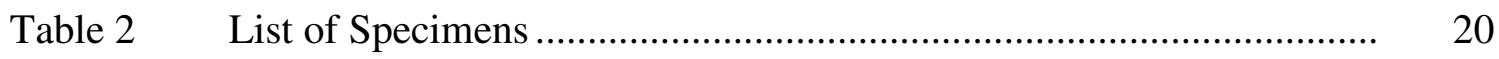

Table $3 \quad$ Transducer Characteristics .......................................................... 34 


\section{CHAPTER I}

\section{INTRODUCTION}

1.1 Motivation and Objective

The National Aeronautics and Space Administration (NASA) is developing a Reusable Launch Vehicle (RLV) technology program with the primary aim of lowering the cost of access to space. Towards this aim, the RLV program seeks the development of a low-cost, reusable, safe and reliable single-stage-to-orbit (SSTO) launch vehicle $[1,2]$. The most important factor towards lowering the cost of access to space is the reduction in the structural weight of the RLV. The use of light-weight, highstrength graphite/epoxy composite materials is being looked upon as the primary means of achieving this weight reduction. Accordingly, extensive use of graphite/epoxy composites is envisioned in the next generation RLV structural components.

The cryogenic fuel tank is one of the largest structural components in a RLV and the design of a large-scale, light-weight cryogenic tank is considered to be one of the most challenging aspects of RLV design [1-3]. The fuel tanks are being fabricated using graphite/epoxy composite laminates and will be required to contain liquid hydrogen $\left(\mathrm{LH}_{2}\right)$ propellant. At $\mathrm{LH}_{2}$ temperature $\left(-252.8{ }^{\circ} \mathrm{C}, 20.2 \mathrm{~K}\right)$, most composite materials will develop matrix cracks due to the high thermal stresses and decrease in the stresses required to produce matrix cracks.

This thesis follows the style and the format of Journal of Applied Mechanics. 


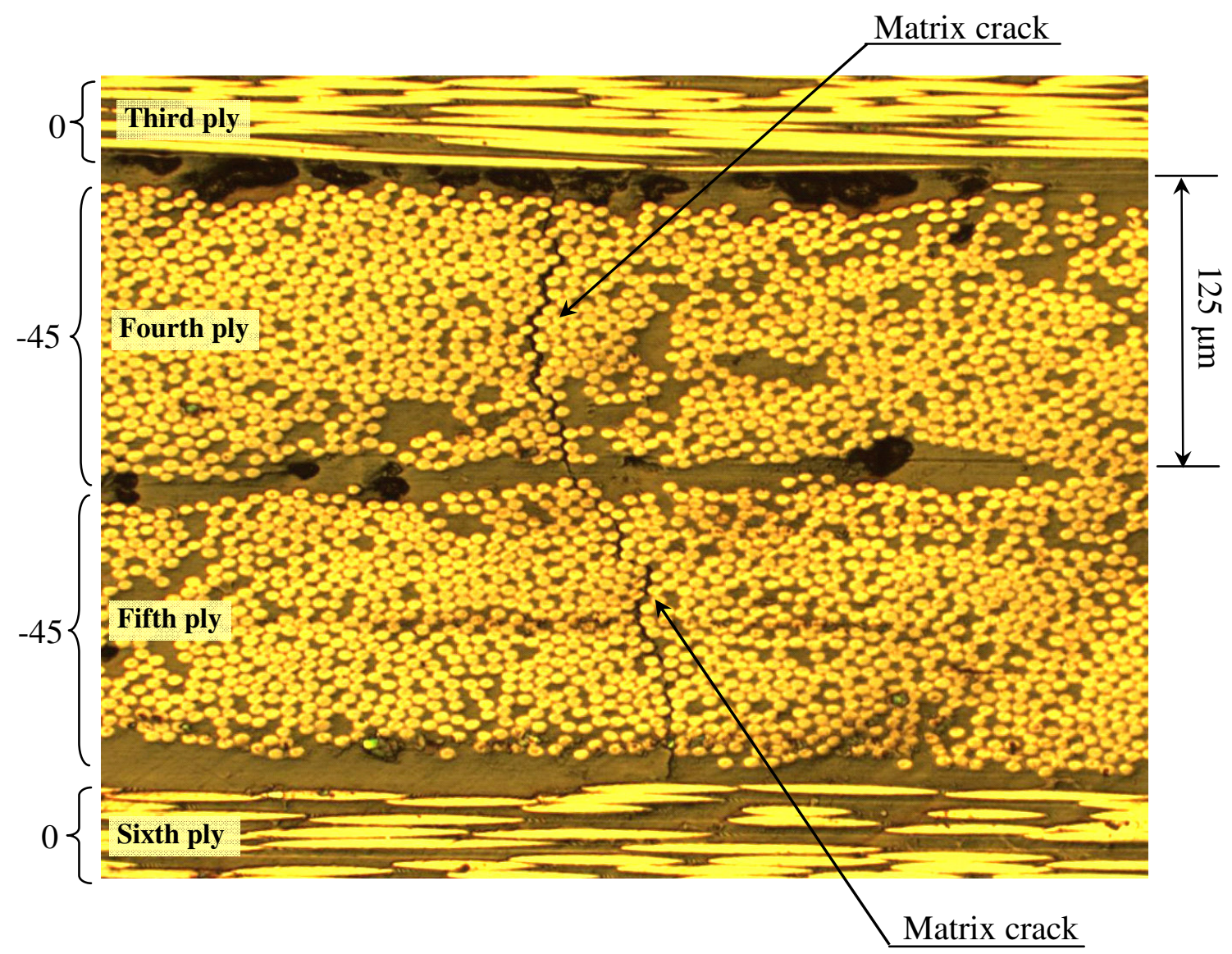

Fig. 1.1 Optical micrograph showing matrix cracks in the fourth and the fifth ply of an eight-ply [90/45/0/-45]s graphite/epoxy laminate. 
A photograph of a part of the edge of an eight-ply [90/45/0/-45]s graphite/epoxy composite laminate is shown in Fig. 1.1; a matrix crack is clearly visible. A build up of matrix cracks in all plies of a laminated composite wall of a tank may lead to the formation of a network of interconnected cracks through the thickness of the tank-wall and provide a path for fuel to permeate to the outside [3-7]. The ignition energy of hydrogen in air is extremely low; $0.02 \mathrm{~mJ}$ [8]. A concentration of $4 \%$ (or more) of hydrogen in air is flammable [8,9]. Therefore the leakage of hydrogen to atmosphere may lead to extremely dangerous situations. In order to ensure the safety of the RLVs, it is critical to accurately predict the fuel leakage from the tank to the outside in order to ensure that it does not leak above a certain acceptable limit even after several launches of the space vehicle. Some elementary models have been developed to predict the permeability of a cryogenic propellant through the matrix cracks in a damaged composite laminate; see, for example, Aoki et al. [4], McManus et al. [6] and Kumazawa et al. [7]. These models require an a priori knowledge of the matrix cracks in all the plies and the interconnectivity between them.

The presence of matrix cracks is a necessary but not a sufficient condition for leakage to occur through the thickness of a laminate. This is explained with the help of Fig. 1.2. As an illustrative example, consider a three-ply [90/0/90] laminate with just one crack in each ply. The cracks are arranged in such a way as to allow a continuous path for flow of the propellant from the upper side of the laminate to the lower side, as shown schematically in Fig. 1.2(a). Now, consider a situation in which two of the plies contain a large number of matrix cracks, but one ply (the middle ply) is undamaged as 
(a)
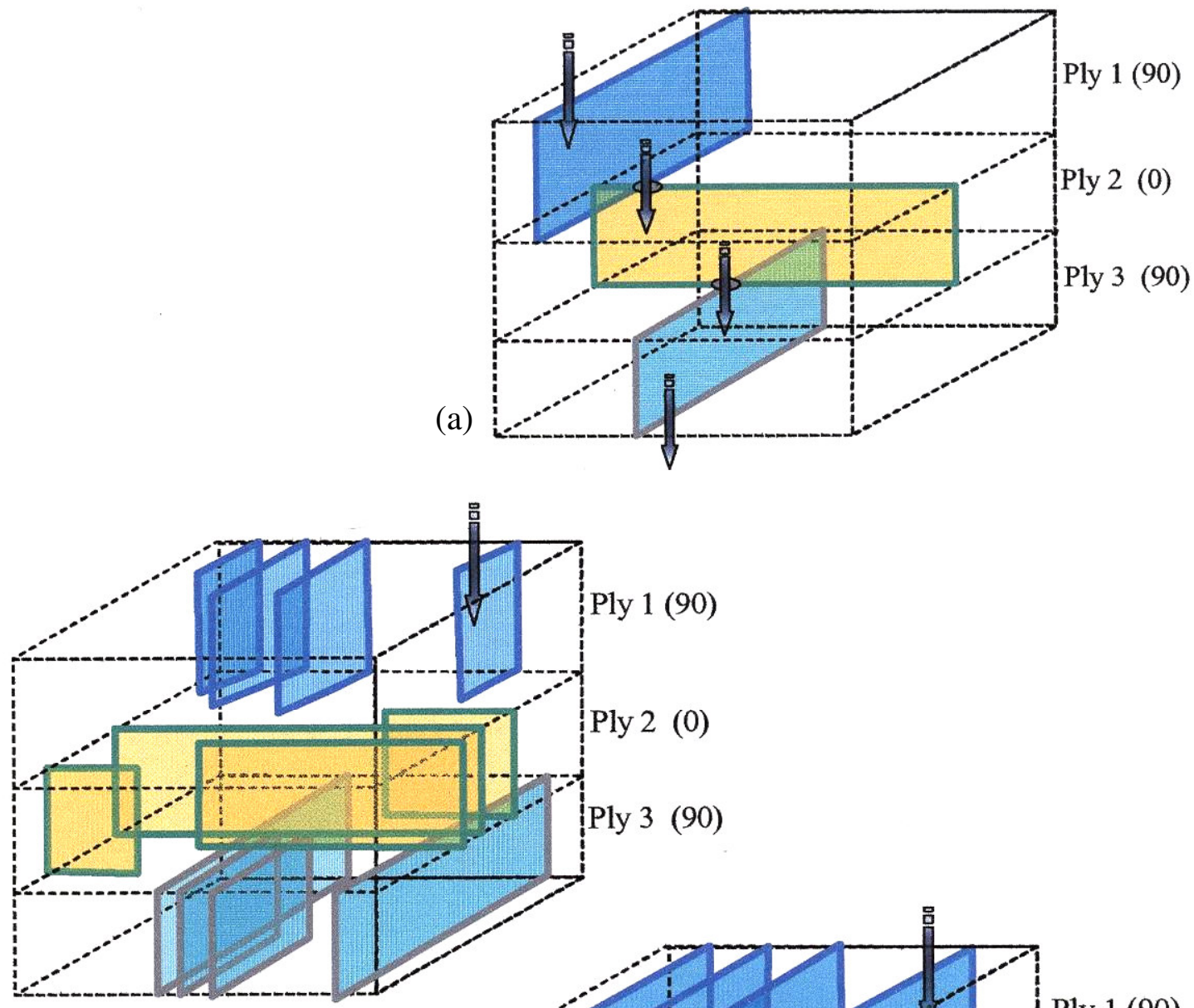

(b)

Ply $1(90)$

Ply 2 (0)

Ply 3 (90)

(c)

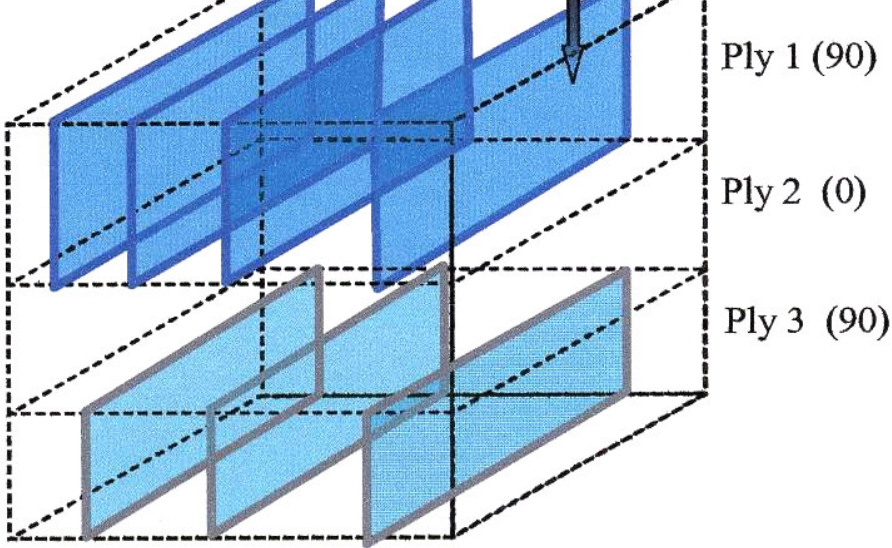

Fig. 1.2 A schematic of the possible arrangements of matrix cracks in a damaged [90/0/90] laminate. (a) Interconnected cracks; leakage occurs (b) no cracks in the second ply; leakage does not occur (c) cracks in the first ply do not intersect cracks in the second ply; leakage does not occur. 
represented in Fig. 1.2(b). Evidently, there will be no leakage through the laminate thickness in this case. Finally, consider a situation where all three plies contain matrix cracks but there is not even a single intersection between the cracks in the first ply and those in the second ply; this is illustrated in Fig. 1.2(c). Once again, there will be no leakage across the laminate. Evidently, in addition to the density of the cracks, the extent and the spatial arrangement of the cracks in each ply is also required for the prediction of permeability. This defines the objective of this thesis: the development of an ultrasonic ply-by-ply NDE technique for the detection of matrix cracks in laminated composites.

\subsection{Overview of Techniques Used for the Detection of Matrix Cracks in Composites}

\subsubsection{Optical Microscopy}

Due to its simplicity, optical microscopy has been one of the most popular techniques for the nondestructive evaluation of damage in composites. Extensive use of this technique is found in literature reporting the study of the damage initiation and progression in composites due to fatigue loading. However, in certain situations, the technique fails to provide reliable results. This is discussed in the following.

For the preciseness of communication, the following terms are defined: (1) a "through crack" is defined as a crack that traverses the entire width of a specimen and therefore it is optically visible at both edges; (2) a "partial crack" is defined as a crack that intersects only one of the edges (only visible at one edge) and (3) an "internal crack" is defined as a crack that does not intersect either of the edges (an optical examination will completely miss this crack). A schematic illustration of a through crack, a partial 


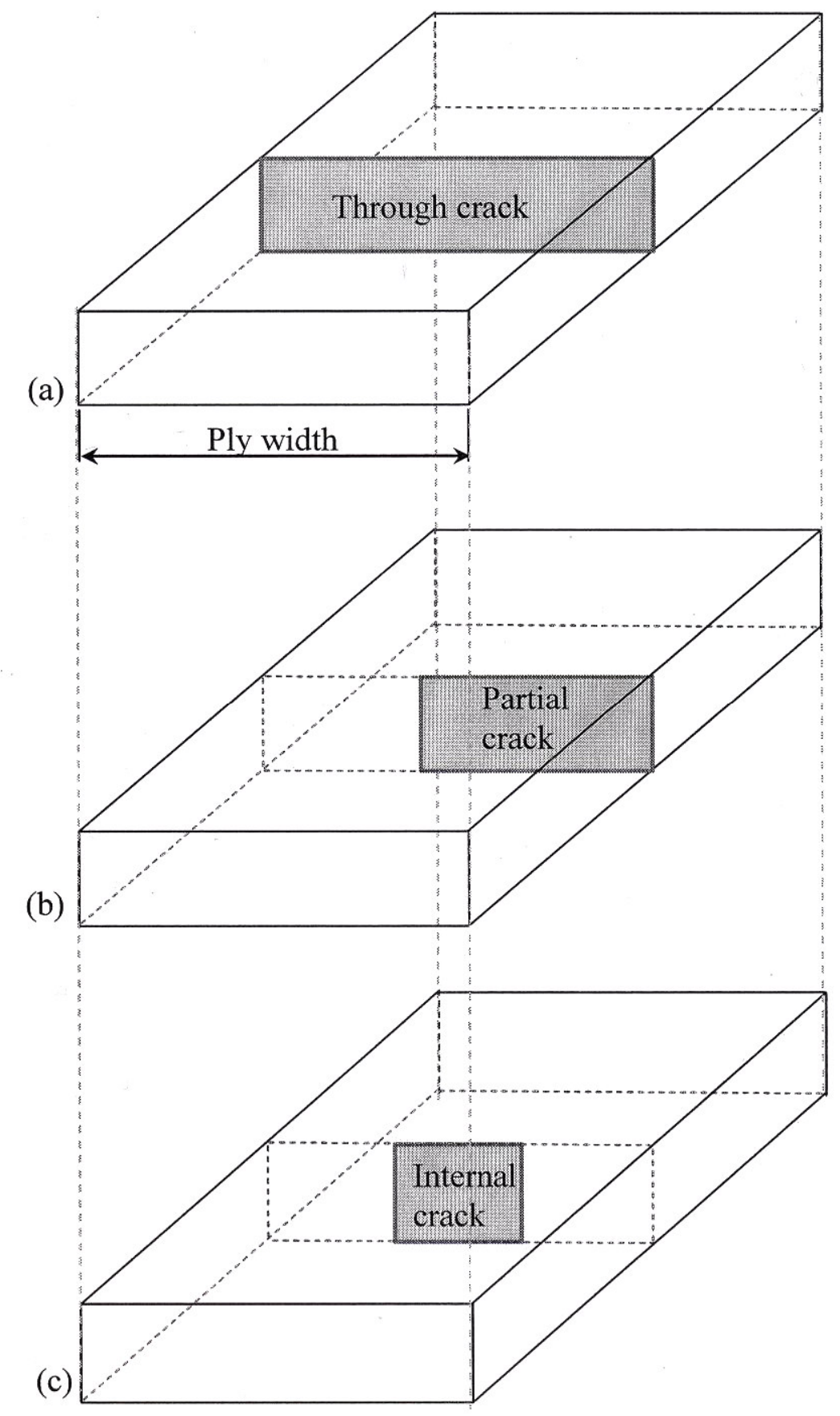

Fig. 1.3 A schematic illustrating (a) a through crack, (b) a partial crack and (c) an internal crack in a single ply. 
crack and an internal crack is shown in Figs. 1.3(a), (b) and (c), respectively. Using ultrasonic NDE, X-radiography, optical microscopy, and by cutting the specimens (destructive evaluation), Maslov et al. [10] have reported the existence of through cracks, partial cracks and internal cracks in three-ply [0/90/0] carbon-fibre/bismaleimide laminates. Consequently, techniques like optical microscopy (including edge replication), which rely solely on the visual examination of the laminate edges for crack detection, will miss the cracks that do not run to the edge under examination. Figure 1.4 illustrates this drawback of the optical microscopy technique in detecting matrix cracks in an eight-ply graphite/epoxy (IM7/5250) composite laminate with a [90/45/0/-45] s stacking sequence. Edges 'A' and 'B' were examined under a high-power microscope at 200X magnification over a $30 \mathrm{~mm}$ length of the damaged laminate. Cracks in only the first ply $\left(90^{\circ}\right.$ orientation) were recorded. The locations of these cracks at the edges are schematically represented by vertical lines drawn above edge 'A' and below edge 'B' respectively. Of the 18 intersections of the matrix cracks with the edge ' $\mathrm{B}$ ' and six intersections with the edge ' $\mathrm{A}$ ', only three correspond to through cracks (T); the remaining correspond to partial cracks $(\mathrm{P})$. In situations such as this, optical microscopy will lead to a grossly incorrect determination of the matrix cracks.

\subsubsection{Penetrant Enhanced X-radiography}

Penetrant enhanced X-radiography is often considered as the best NDE tool for the detection of matrix cracks in composites. The technique employs the infiltration of a contrast-enhancing, X-ray opaque liquid into the matrix cracks in a composite, in 


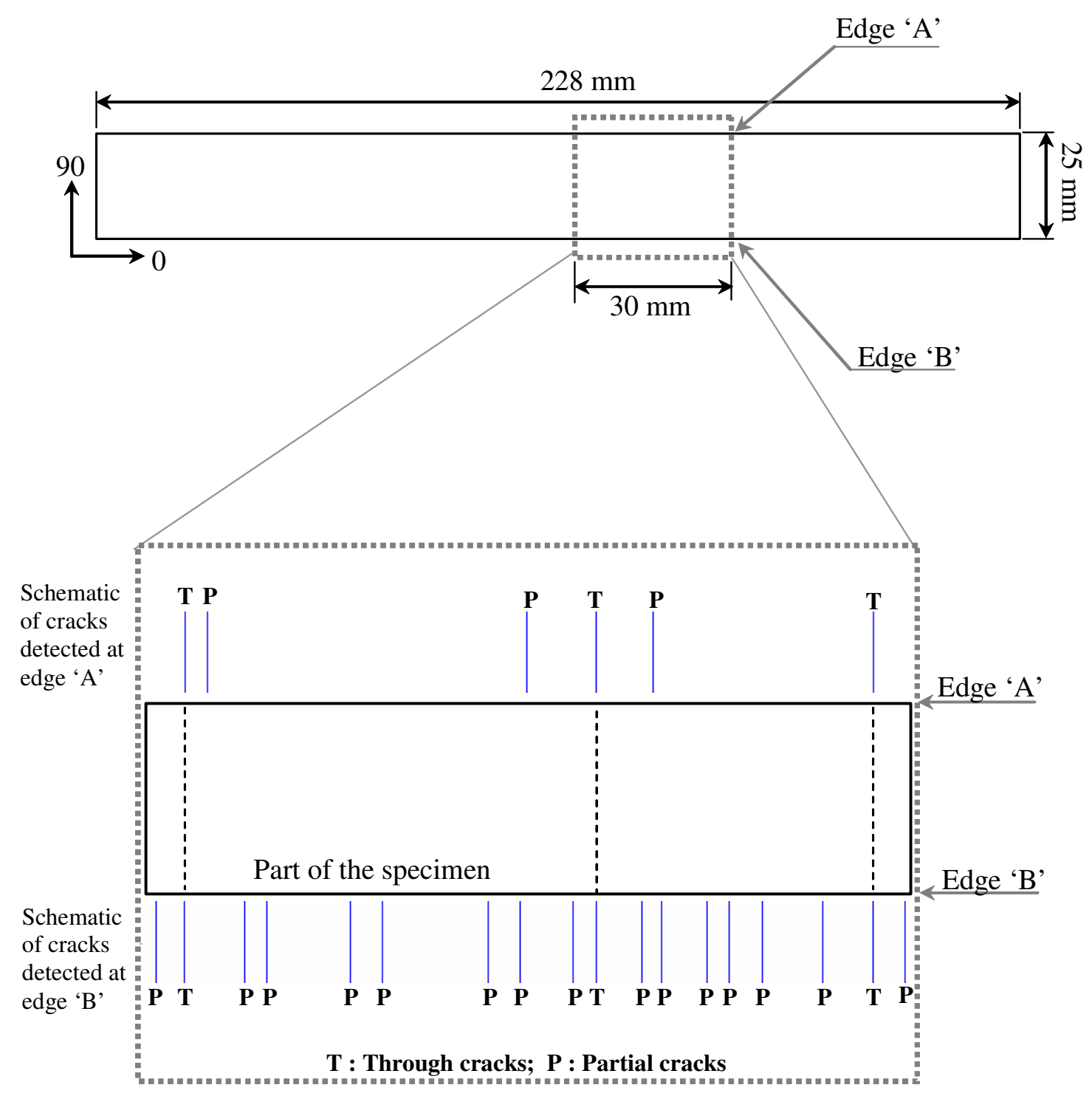

Fig. 1.4 A schematic showing the cracks in the first ply $\left(90^{\circ}\right)$ of a $[90 / 45 / 0 /-45]_{\mathrm{s}}$ graphite/epoxy laminate as detected by optical microscopy. 
conjunction with low energy X-rays (usually between $10-50 \mathrm{kV}$ ) [11]. With the proper choice of penetrant and under optimal X-ray settings, the method has been known to provide excellent resolution for the detection of matrix cracks. However, this method is successful only when there exist continuous paths from the surface to the matrix cracks to allow the inflow of an X-ray opaque penetrant to the cracks. Conversely, the internal cracks, where the penetrant cannot reach, will go undetected. A major drawback of Xradiography is that, from an X-ray image, it is not possible to distinguish between cracks in two different plies of a composite laminate having the same orientation. For example see Fig. 1.5; an X-ray image of highly damaged eight-ply laminate having a [90/45/0/45] lay-up, showing matrix cracks. In this case, one cannot distinguish between cracks in the third ply $\left(0^{\circ}\right)$ from cracks in the sixth ply $\left(0^{\circ}\right)$ having the same orientation, or cracks in the second ply $\left(45^{\circ}\right)$ from cracks in the seventh ply $\left(45^{\circ}\right)$ having the same orientation. Thus, given an X-ray image, it is impossible to obtain ply-by-ply information about matrix cracks. In the classical damage mechanics, this does not present a problem. However, from the point of view of prediction of permeability as discussed in the foregoing (see Fig. 1.2), this becomes a serious drawback.

It should also be noted that the aforementioned techniques of optical microscopy and X-radiography become impractical in real-life applications for the following reasons. Since optical microscopy relies entirely on the availability of edges, it becomes ineffective in the absence of access to the edges. For example, a cylindrical cryogenic composite tank may not have any edges available for optical examination. Xradiography is severely restricted by the shape and size of the specimen, and also by the 


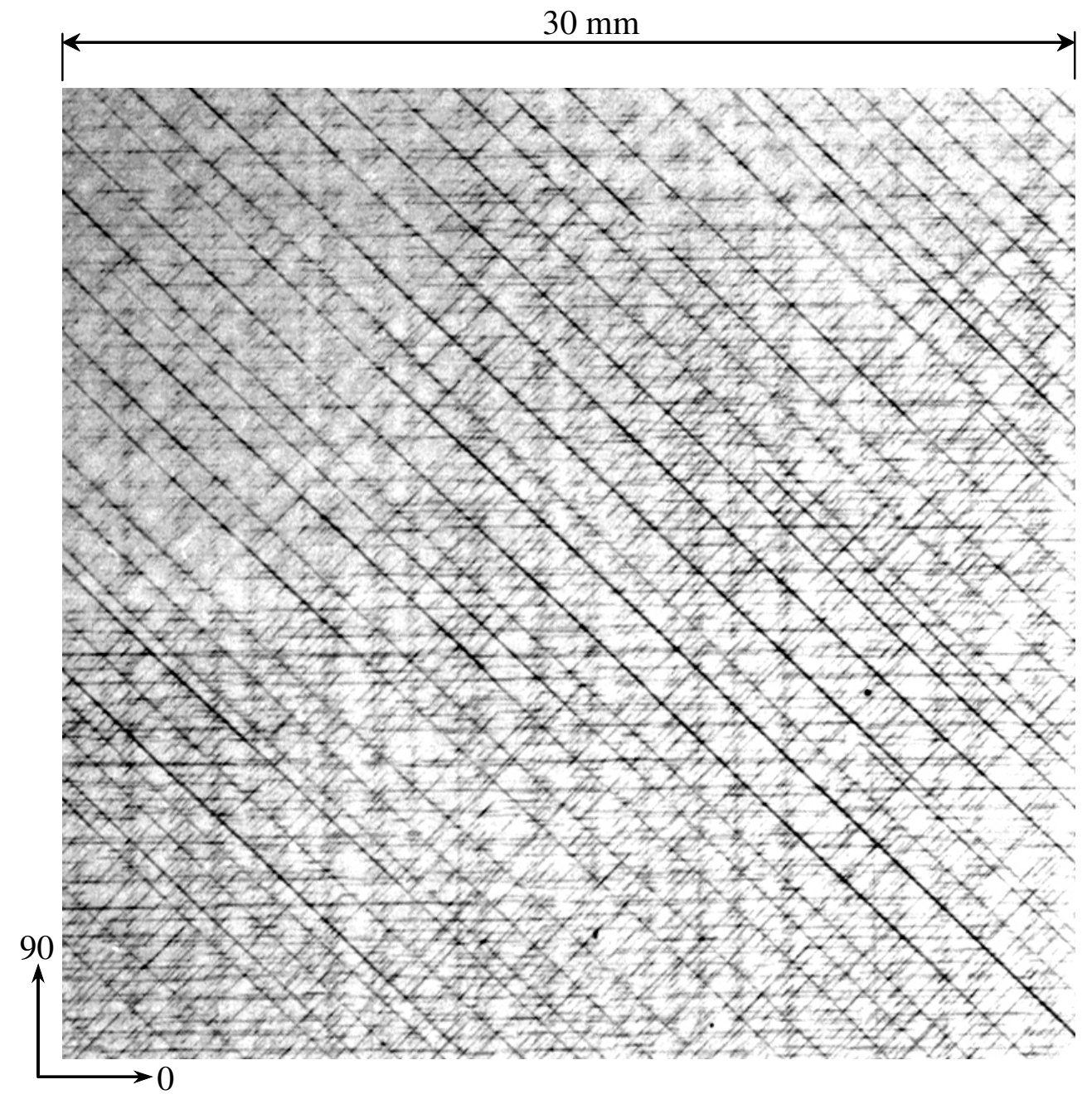

Fig. 1.5 X-ray image showing matrix cracks (dark lines) in a an eightply $[90 / 45 / 0 /-45]_{s}$ graphite/epoxy laminate. 
safety and environmental concerns regarding halogenated hydrocarbon penetrants [11]. However, both these methods serve as excellent calibration tools for laboratory work.

\subsubsection{Ultrasonics}

Currently, ultrasonic nondestructive evaluation is the most extensively used method for the detection of damage in composite materials in the industry. It provides a truly non-invasive method for damage detection and unlike optical microscopy and Xradiography, its success does not depend on the accessibility to specimen edges.

The basic concept behind ultrasonic nondestructive evaluation may be explained as follows. With the help of a transducer, ultrasonic waves are generated and made incident on the specimen under investigation. The waves then propagate through the material and respond to the presence of flaws, elastic properties and geometry of the specimen. A receiving transducer detects the waves emerging from the specimen which are then analyzed to characterize the defects and mechanical properties. Figure 1.6 shows the schematic of a typical experimental setup for the ultrasonic detection of a delamination as an example.

A variety of ultrasonic techniques, as differentiated by the orientation of the transmitting and receiving transducers, and mode of wave propagation, are available for the detection of damage in composite materials. The choice depends mainly on the size and the orientation of the flaw to be detected, and the elastic properties of the material. In the following, the discussion of ultrasonic techniques is confined to the detection of 


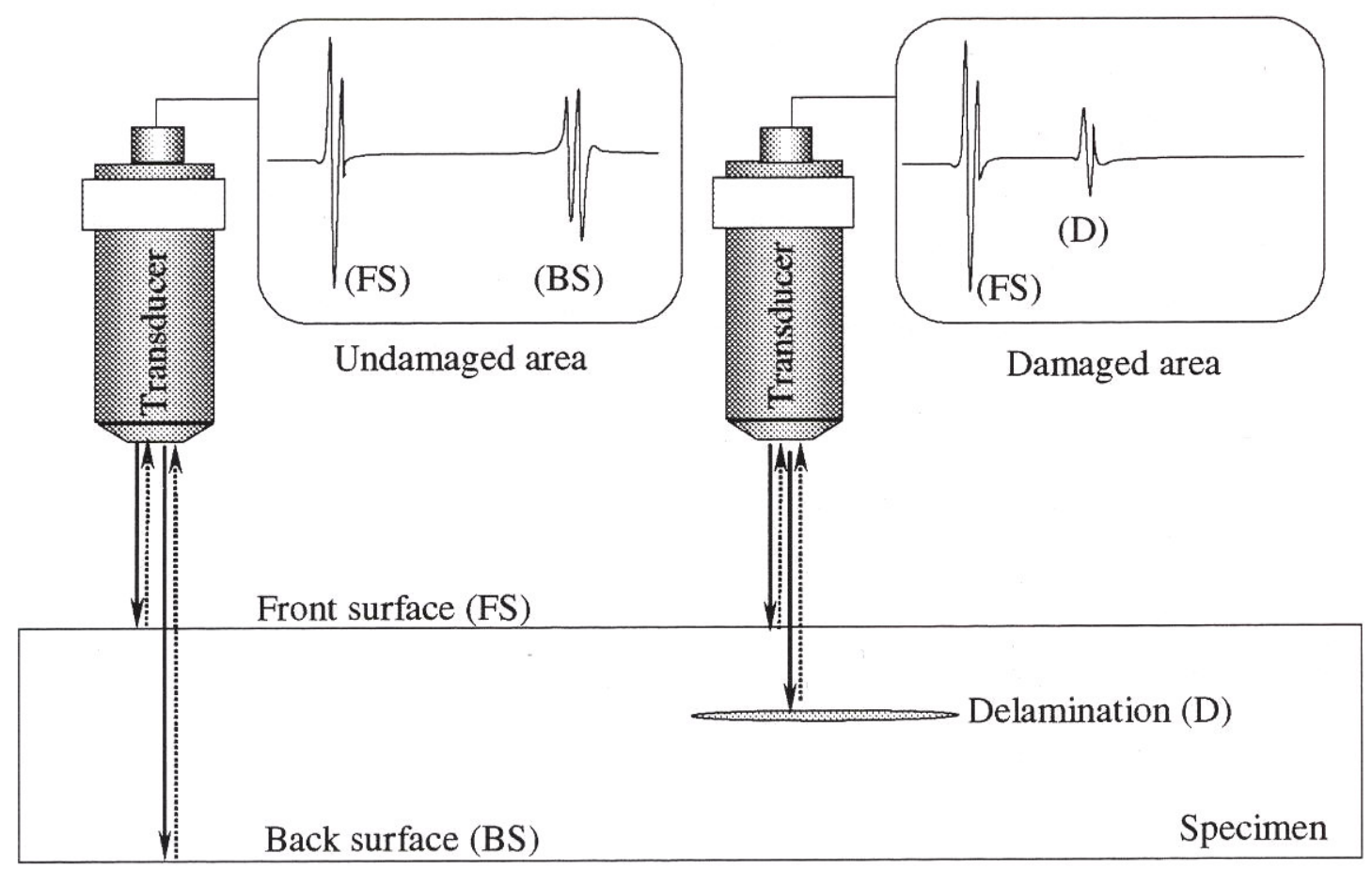

Fig. 1.6 A schematic of a typical ultrasonic nondestructive evaluation setup. 
matrix cracks in laminated composites. For a detailed discussion on the subject of ultrasonic nondestructive evaluation, the readers may refer to Krautkramer and Krautkramer [12] and Shull [13].

\section{Normal Incidence Ultrasonics}

This technique employs the scanning of a specimen with a normally incident ultrasonic transducer in the pulse-echo mode or the through-transmission mode. A focused or a flat transducer may be used. Traditionally, this technique has been used for the detection of defects which are in the plane of the laminate and perpendicular to the incident ultrasonic waves; for example, delaminations. Normal incidence is considered unsuitable for the detection of matrix cracks since the cracks are parallel to the incident waves; see Fig. 1.7. However, some researchers have used it for detection of matrix cracks in cross-ply laminates by way of measuring the attenuation of ultrasonic waves caused by the presence of the cracks; see, for example, Wooh and Daniel [14], Moran et al. [15] and Kaczmarek [16]. In Wooh and Daniel [14], results using normal incidence have been reported only for 40 -ply thick $\left[0_{10} / 90_{10}\right]_{\mathrm{s}}$ laminates with cracks having cracklength $2.54 \mathrm{~mm}$ in the $90_{20}$ ply-group. Moran et al. [15] tested specimens with lay-ups $\left[90_{4} / 0_{4}\right]_{\mathrm{s}}$ with cracks in both the $90_{4}$ ply-groups; however, the results reported using the normal incidence technique did not distinguish between the cracks in the two ply-groups. Kaczmarek [16] tested $\left[0_{2} / 90_{2}\right]_{\mathrm{s}}$ laminates with cracks in the $90_{4}$ ply-group and $\left[90_{2} / 0_{2}\right]_{\mathrm{s}}$ with cracks in both the $90_{2}$ ply-groups. In this work too, for the $\left[90_{2} / 0_{2}\right]_{\mathrm{s}}$ laminates, the results did not distinguish between the cracks in the two distinct $90_{2}$ ply-groups. 


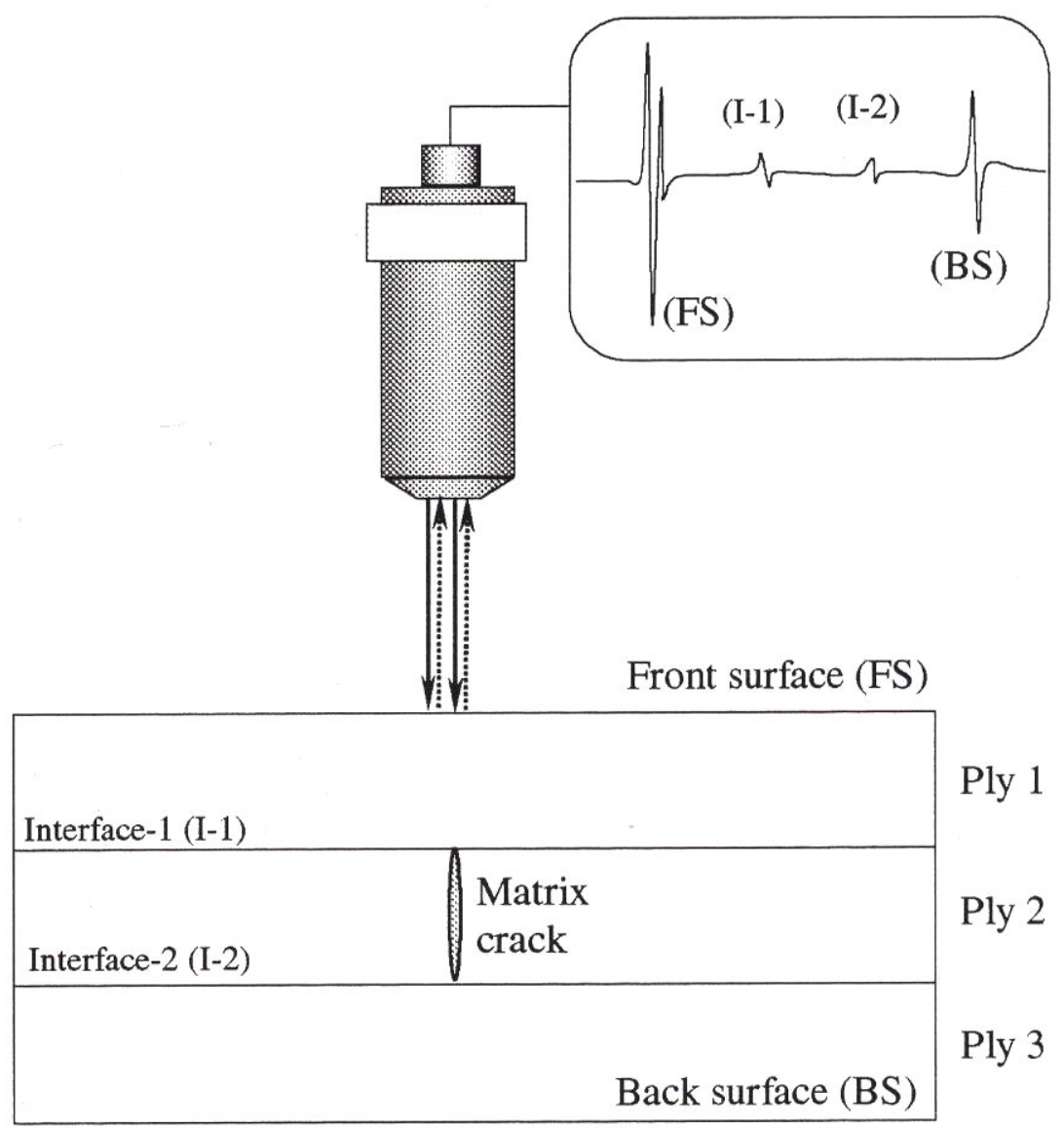

Fig. 1.7 A schematic of setup for the detection of matrix cracks in a laminated composite using the normal incidence ultrasonic technique. 


\section{Lamb Waves}

Lamb waves are waves propagating in the plane of a plate with traction free boundaries. As is well known, the particle displacement is in two directions, namely, the direction of wave propagation (in the plane of the plate) and the thickness direction (perpendicular to the plane of the plate). When the plate is immersed in a fluid, the Lamb waves leak acoustic energy into the surrounding fluid. Therefore such waves have been named as "Leaky Lamb Waves". Conventional nondestructive evaluation techniques using leaky lamb waves measure the speed and attenuation of these waves to characterize the damage state of a material (specifically, the linear crack density in composites); see for example, Dayal and Kinra [17,18], Bar-Cohen, Lih and Mal [19]. However, in these works individual cracks in composite laminates were not detected. Recently, Maslov et al. [10] have used leaky lamb waves to detect individual cracks in carbon-fibre/bismaleimide (IM7/5250-4) composite laminates. However, results were reported only for a sparsely damaged [0/90/0] laminate, with cracks in only one ply $\left(90^{\circ}\right)$

\section{Polar Backscattering}

The polar backscattering technique was first introduced by Bar-Cohen and Crane [20] in the context of the backscattered signal observed due to the presence of fibers by an obliquely incident transducer. Since the pioneering work of Bar-Cohen and Crane [20], preliminary results demonstrating the use of ultrasonic polar backscattering for the detection of matrix cracks in laminated graphite/epoxy composites have been reported 
by several other researchers [14,15,20-24]. Wooh and Daniel [14] used the backscattering technique to detect cracks in fatigued-damaged $90_{4}$ ply group of $\left[0 / 90_{2}\right]_{\mathrm{s}}$ cross-ply specimens. Moran et al. [15] examined cross-ply laminates, namely, $\left[90_{4} / 0_{4}\right]_{\mathrm{s}}$. Their work was limited to detecting cracks of rather large crack-length (4-ply or $0.5 \mathrm{~mm}$; see figure on page 42 for the definition of "crack length") in the $90_{4}$ ply-group which, as we shall see, are much easier to detect than cracks with crack length extending through only one ply or $0.125 \mathrm{~mm}$. Bar-Cohen and Crane [20] examined only sparsely damaged quasi-isotropic, $[0 / \pm 45 / 90]_{\mathrm{s}}$ laminates; of the eight plies, cracks were reported in only the $90_{2}$ ply-group and one of the $45^{\circ}$ plies. Similarly, Raju [21] has reported results for rather large cracks in the $90_{4}$ ply-groups in $\left[90_{4} / 0_{4}\right]_{\mathrm{s}}$ and $\left[0_{2} / 90_{2}\right]_{\mathrm{s}}$ laminates. Gorman [22] examined three different types of laminates: $[ \pm 45]_{\mathrm{s}},[0 / 90]_{\mathrm{s}},\left[0 / 90_{2}\right]_{\mathrm{s}}$; the cracks were present in only one ply-group, namely, $-45_{2}, 90_{2}$, and $90_{4}$, respectively. Steiner et al. [23] used the technique to detect cracks in a cross-ply $\left[0_{2} / 90_{2}\right]_{\mathrm{s}}$ laminate and quasi-isotropic $[45 / 90 /-45 / 0]_{2 s}$ and $\left[45_{2} / 90_{2} /-45_{2} / 0_{2}\right]_{\mathrm{s}}$ laminates. For the quasi-isotropic laminates, the work does not distinguish between cracks in different plies/ply-groups and for the crossply laminates cracks are reported only in the $90_{4}$ ply-group. Aymerich et al. [24] used the backscattering technique for studying damage in quasi-isotropic composites subjected to low-velocity impacts. Their main emphasis was on the detection of delaminations even though they did detect matrix cracks. None of the works reviewed reported a ply-by-ply detection of matrix cracks when all the plies of a composite laminate were highly damaged. Although the backscattering method has been successfully employed to find matrix cracks, so far as we know, no attempt has been made to offer a theoretical wave- 
propagation based explanation of the mechanism of crack detection. It should also be noted that, although much of the laboratory work has been carried out with cross-ply laminates, such laminates are seldom used in real life structures.

\subsection{Scope of This Thesis}

The current NDE work was conducted as a part of an effort, initiated by the National Center for Advanced Manufacturing (NCAM), towards the prediction of permeability of $\mathrm{LH}_{2}$ through composite laminates. Eight-ply quasi-isotropic laminated composite specimens were subjected to thermo-mechanical loading at cryogenic temperatures at the NASA Marshall Space Flight Center (NASA-MSFC), Huntsville, Alabama, to collect permeability data at various loading levels. The specimens were then shipped to Texas A\&M University, College Station, for nondestructive evaluation. In addition to the aforementioned specimens, an 18-ply laminate, subjected to thermomechanical loading at Lockheed Martin, was also nondestructively tested. Details about the specimens are discussed in Chapter II. Loading conditions and related procedures are proprietary information of NASA and Lockheed Martin for their respective specimens and the discussion on the topic will be limited subject to International Traffic in Arms Regulations (ITAR).

As seen in the previous chapter, much of the reported research concerning the detection of matrix cracks in laminate composites has been conducted either on cross-ply laminates containing cracks in only one or two plies. Such laminates are seldom used in 
real structures. In this work, the specimens tested are representative of those proposed for the use in RLV, in terms of stacking-sequence and thickness of individual plies.

The focus of this thesis is the development of the ultrasonic back-scattering technique for finding matrix cracks in each of the plies of a laminated composite even in the presence of a rather high density of cracks in all of the remaining plies. Experimental results are presented for quasi-isotropic laminates, with different lay-ups and laminate thicknesses. Details about the specimen dimensions, lay-up and material system are discussed in Chapter II. In the case of the quasi-isotropic laminates tested, with one exception, all the cracks were one ply $(0.125 \mathrm{~mm})$ long. The ultrasonic data were calibrated by comparing them with the corresponding results obtained by using the traditional methods of optical microscopy and X-radiography. Excellent quantitative correlation was observed between the results obtained with ultrasonics and the traditional methods. Calibration results are presented in Chapter II. 


\section{CHAPTER II}

\section{EXPERIMENTAL PROCEDURES}

\subsection{Specimens and Materials}

In the developmental stages, two cross-ply graphite/epoxy laminates containing matrix cracks were used to calibrate the ultrasonic backscattering technique for the detection of matrix cracks. The details of these cross-ply laminates are listed in Table 1. The ultrasonic technique was then used for the ply-by-ply detection of matrix cracks in five graphite/epoxy laminates with various lay-ups and material systems. These specimens are listed in Table 2 .

TAMU-1 through TAMU-4 specimens were cut from four different $508 \mathrm{~mm}$ (20") diameter composite discs with a quasi-isotropic [90/45/0/-45]s lay-up. These specimens were obtained from NASA-MSFC. Each disc was subjected to $\mathrm{LH}_{2}$ permeability test during which, damage was produced due to the application of cyclic thermo-mechanical loading. The test was stopped when the leakage through the discs exceeded a certain pre-specified limit. The specimens were cut from the discs after the test, for inspection. TAMU-1 was cut in an arbitrary shape that included the circumference of the corresponding disc from an unknown location; a photograph of the TAMU-1 specimen is shown in Fig. 2.1. TAMU-2, TAMU-3 and TAMU-4 were cut in the shape of $25 \mathrm{~mm}$ x $228 \mathrm{~mm}$ (1" x 9") rectangular panels from similar locations on the corresponding discs (Fig. 2.2). Photographs of the TAMU-2, TAMU-3 and TAMU-4 
Table 1 List of Calibration Specimens

\begin{tabular}{|l|l|l|}
\hline $\begin{array}{l}\text { Specimen } \\
\text { Identification }\end{array}$ & Lay-up & $\begin{array}{l}\text { Material } \\
\text { System }\end{array}$ \\
\hline CALB-1 & {$\left[0 / 90_{4}\right]_{\mathrm{s}}$} & AS4/5250-4 \\
\hline CALB-2 & {$\left[0 / 90_{3}\right]_{\mathrm{s}}$} & AS4/5250-4 \\
\hline
\end{tabular}

Table 2 List of Specimens

\begin{tabular}{|l|l|l|}
\hline $\begin{array}{l}\text { Specimen } \\
\text { Identification }\end{array}$ & Lay-up & $\begin{array}{l}\text { Material } \\
\text { System }\end{array}$ \\
\hline TAMU-1 & {$[90 / 45 / 0 /-45]_{\mathrm{s}}$} & IM7/977-2 \\
\hline TAMU-2 & {$[90 / 45 / 0 /-45]_{\mathrm{s}}$} & IM7/977-2 \\
\hline TAMU-3 & {$[90 / 45 / 0 /-45]_{\mathrm{s}}$} & IM7/950-1 \\
\hline TAMU-4 & {$[90 / 45 / 0 /-45]_{\mathrm{s}}$} & IM7/5250-4 \\
\hline TAMU-5 & {$[0 / 45 / 90 /-45 / 0 / 45 / 90 /-45 / 0]_{\mathrm{s}}$} & IM7/977-2 \\
& & \\
\hline
\end{tabular}




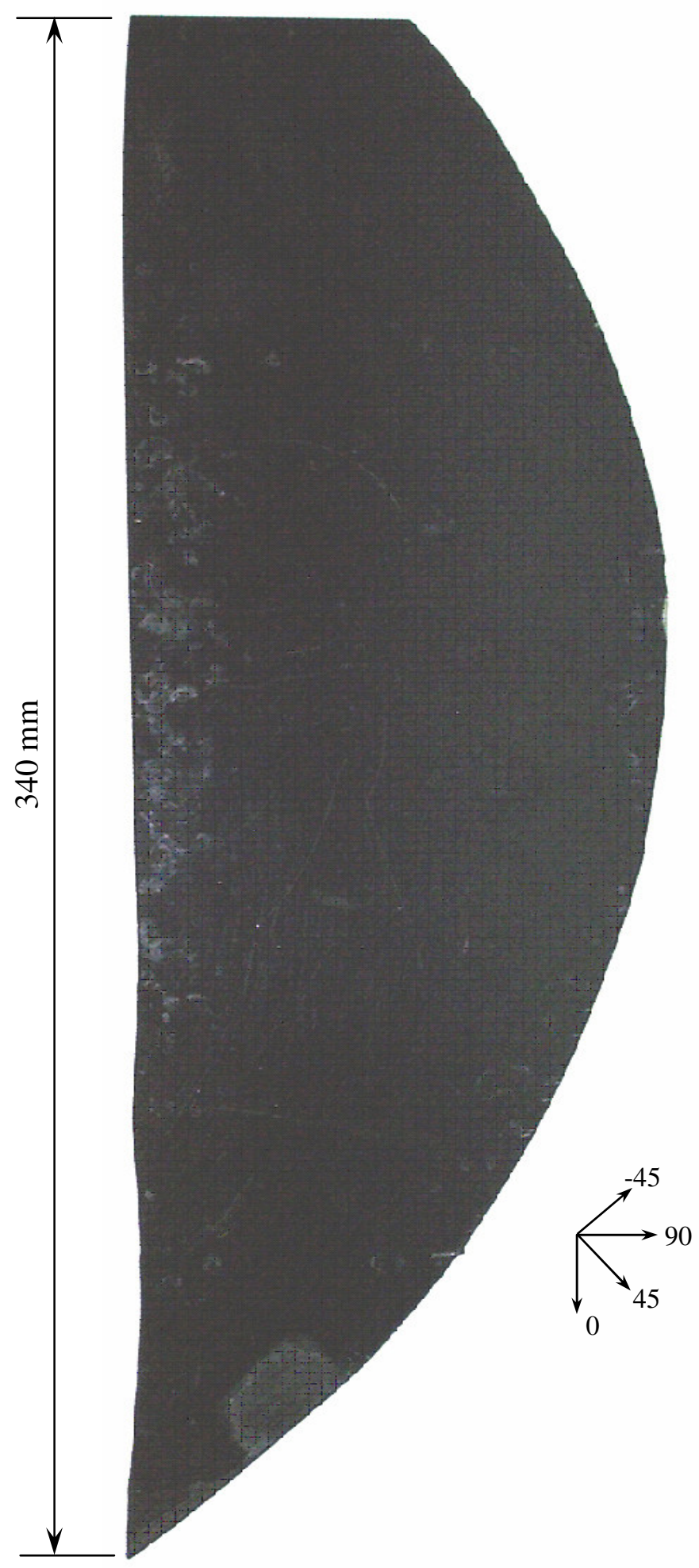

Fig. 2.1 A photograph of the eight-ply TAMU-1 specimen. 


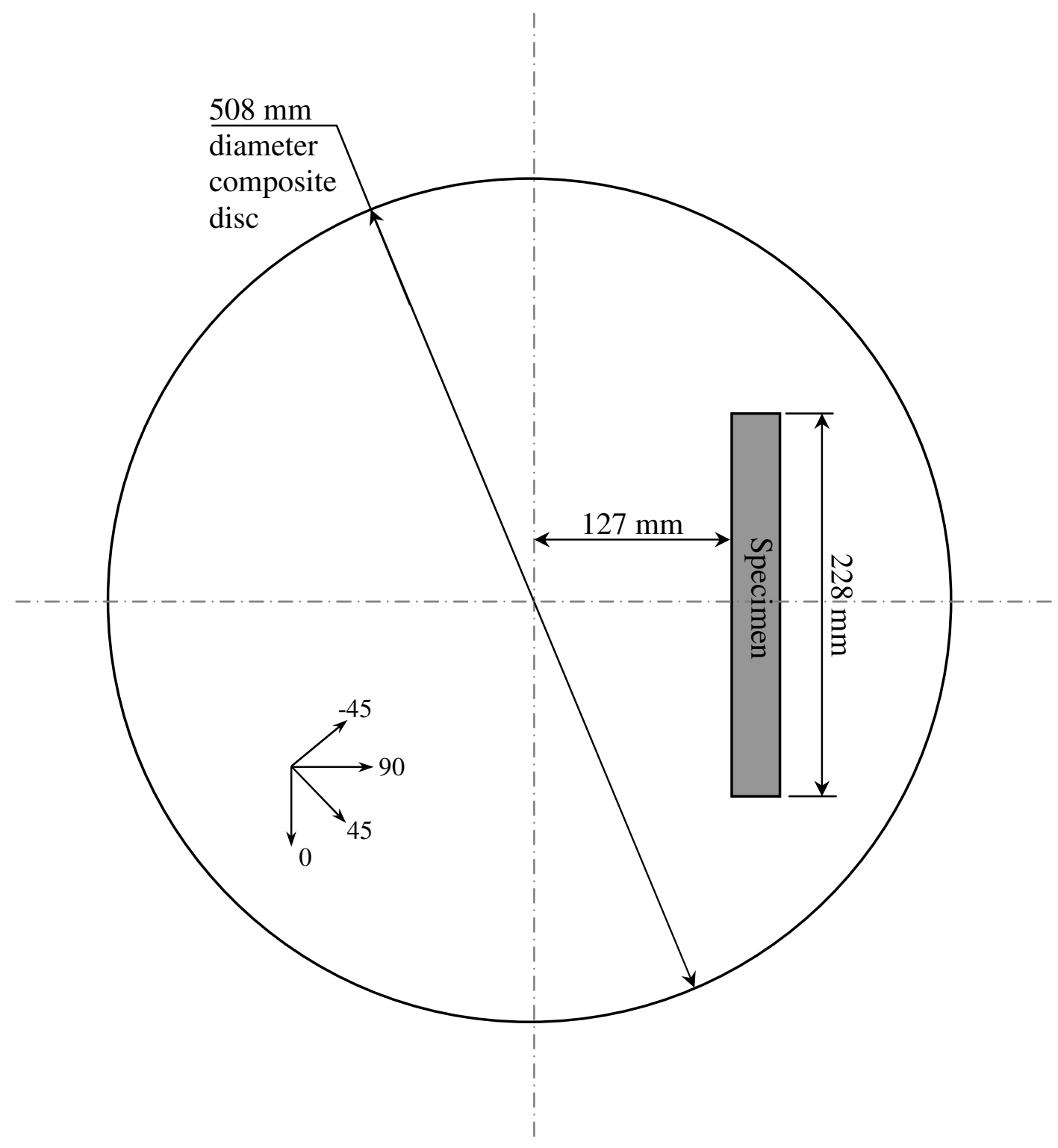

Fig. 2.2 A schematic showing the location of TAMU-2, TAMU-3 and TAMU-4 specimens on the corresponding composite discs. 


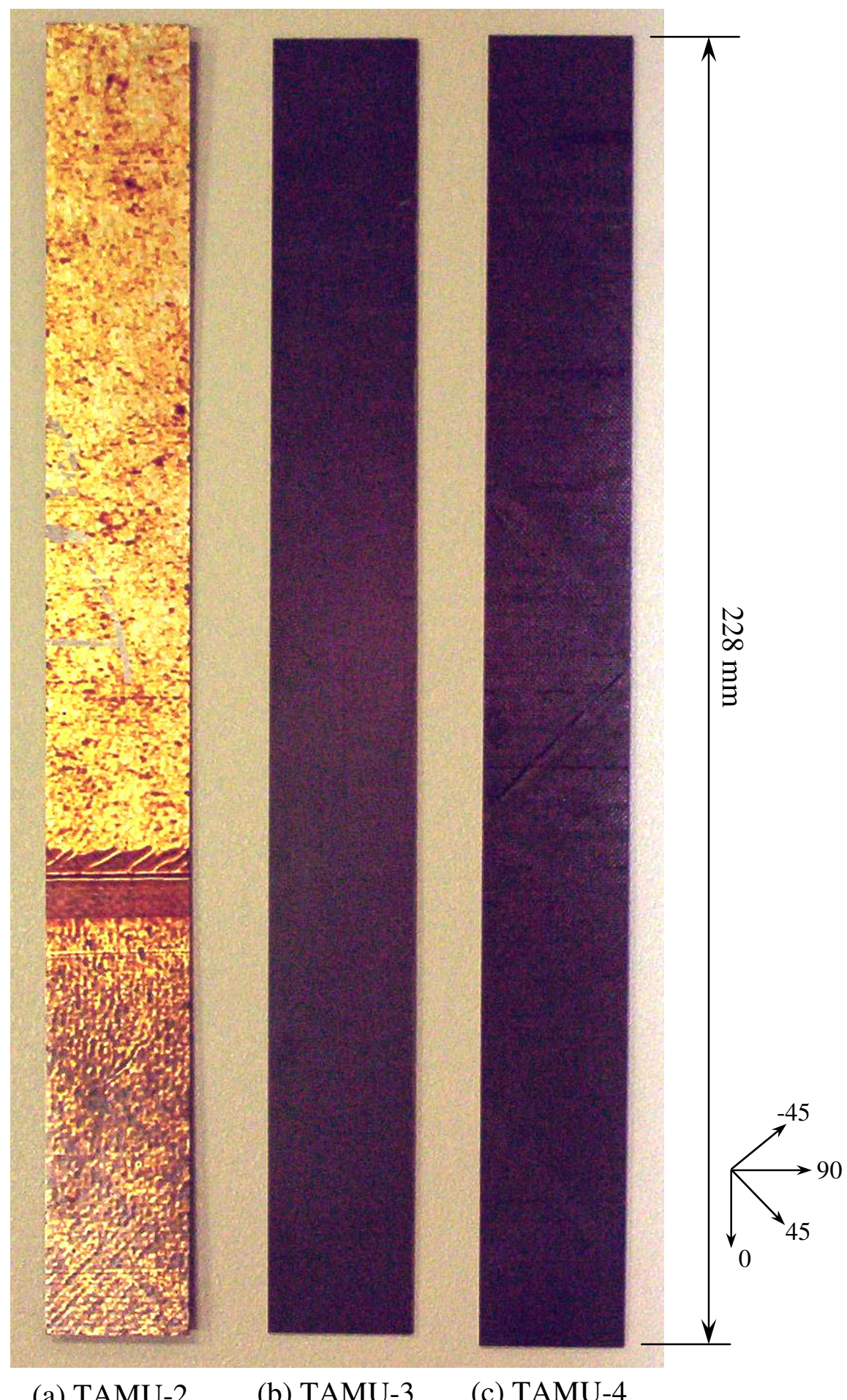

$\begin{array}{lll}\text { (a) TAMU-2 } & \text { (b) TAMU-3 } & \text { (c) TAMU-4 }\end{array}$

Fig. 2.3 Photographs of the TAMU-2, TAMU-3 and TAMU-4 specimens. 


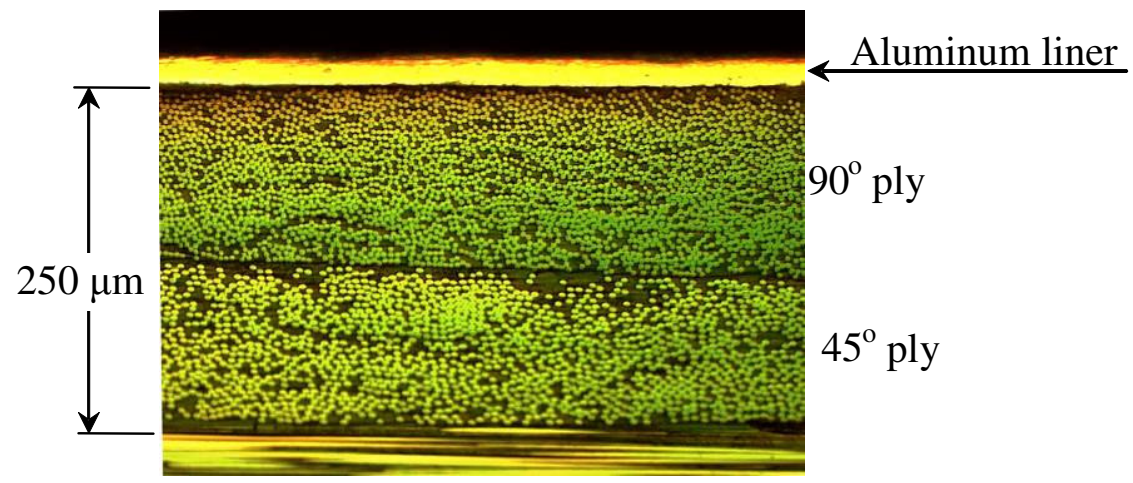

Figure 2.4 An optical micrograph of an edge of the TAMU-2 specimen showing the aluminum liner.

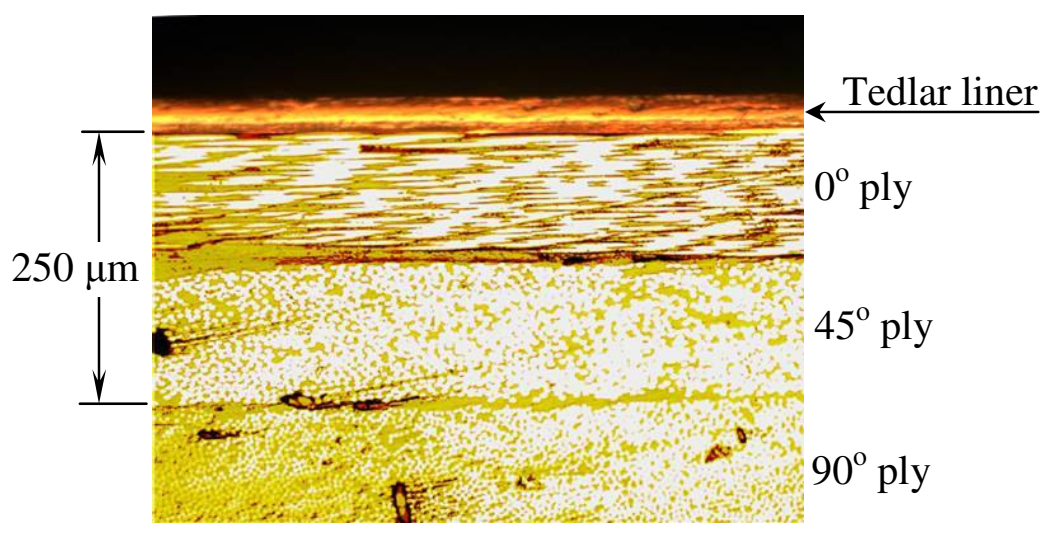

Figure 2.5 An optical micrograph of an edge of the TAMU-3 specimen showing the tedlar (polyvinyl fluoride) liner. 


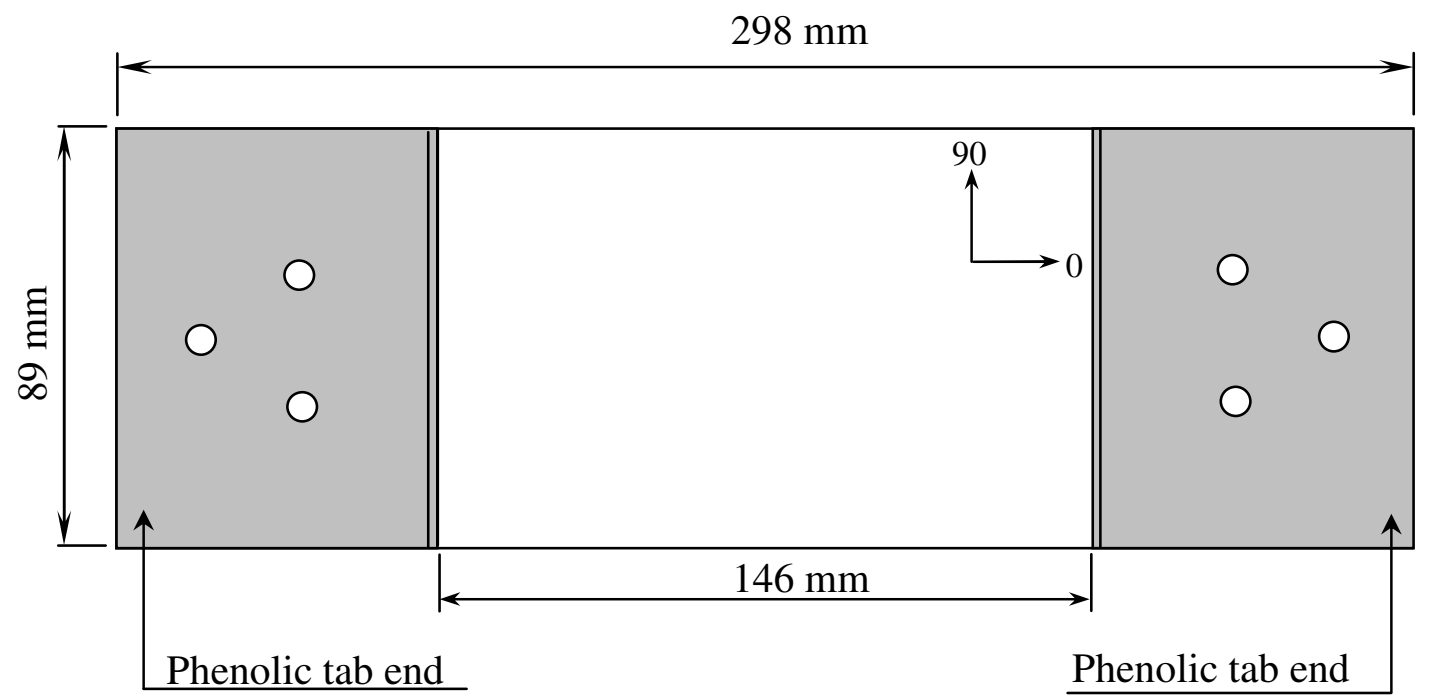

Fig. 2.6 A schematic of the 18-ply TAMU-5 specimen. 
are shown in Fig. 2.3(a), Fig. 2.3(b) and Fig. 2.3(c) respectively. On one side of the TAMU-2 specimen, the surface was covered with a thin aluminum liner, whereas the other side of the specimen presented a rough ply-surface (characteristic of the scrim cloth used during manufacturing of the composite). The thickness of the liner was estimated to be approximately $20 \mu \mathrm{m}$. Optical micrograph of one of the edges of the specimen, showing the aluminum liner, at $200 \mathrm{X}$ magnification, is presented in Fig. 2.4. The TAMU-3 specimen showed similar physical characteristics except that on one surface it had a tedlar (polyvinyl fluoride) liner. Optical micrograph of an edge of the specimen showing the liner is presented in Fig. 2.5. The thickness of the liner was about $25 \mu \mathrm{m}$. TAMU-4 specimen did not have a liner, but both ply-surfaces were rough.

TAMU-5 is an 18-ply [0/45/90/-45/0/45/90/-45/0 $]_{\mathrm{s}}$ laminate in the form of a 89 mm x 298 mm (3.5” x 12”) panel obtained from Lockheed Martin Michoud (LMM), New Orleans, Louisiana. This specimen was subjected to cyclic thermo-mechanical loading at cryogenic temperature during Helium $(4.55 \mathrm{~K})$ permeability tests at the LMM facility. A schematic of this specimen is shown in Fig. 2.6.

\subsection{Optical Microscopy and X-radiography}

Optical Microscopy and X-radiography techniques were used to generate corroborative data to compare the matrix cracks detected by using the ply-by-ply ultrasonic NDE technique. The methodology for these techniques is briefly described in the following. 
Optical microscopy was done by visually inspecting the edges of the specimens under a high power microscope. The details involved in the optical microscopy technique are as follows. The edges of each specimen were polished progressively, first using 600 and 1200 grit sandpapers and then using high purity $0.3 \mu \mathrm{m} \mathrm{Al}_{2} \mathrm{O}_{3}$ (alumina) powder, to get a near optical-quality finish. Then, cracks that were visible at the edges were mapped using an optical microscope (at about 200X magnification) equipped with a motorized positioning stage controlled by a computer. The recorded data files were later used to generate a schematic map of the cracks.

Penetrant enhanced X-radiography was conducted using Diiodomethane (methylene iodide) as X-ray opaque penetrant. The specimens were thoroughly cleaned with 2-propanol (isopropyl alcohol) and then dried in vacuum. Immediately after drying, the specimens were immersed in diiodomethane for about thirty minutes. Next, the excess penetrant was wiped off from the surfaces of the specimens before they were placed directly on top of a Kodak X-OmatTL film pack. The specimens were then exposed to X-rays using a General Electric X-ray machine at $30 \mathrm{kV}$ and $1 \mathrm{~mA}$ for 10 second.

\subsection{Ultrasonic Test Equipment}

All the ultrasonic data in this work were collected using a SONIX FlexSCAN ultrasonic scanning system (SONIX [25]). A schematic of the system is shown in Fig. 2.7. The setup consists of an immersion tank fitted with a scanning carriage platform. The motorized platform is computer-controlled and is capable of moving an ultrasonic 


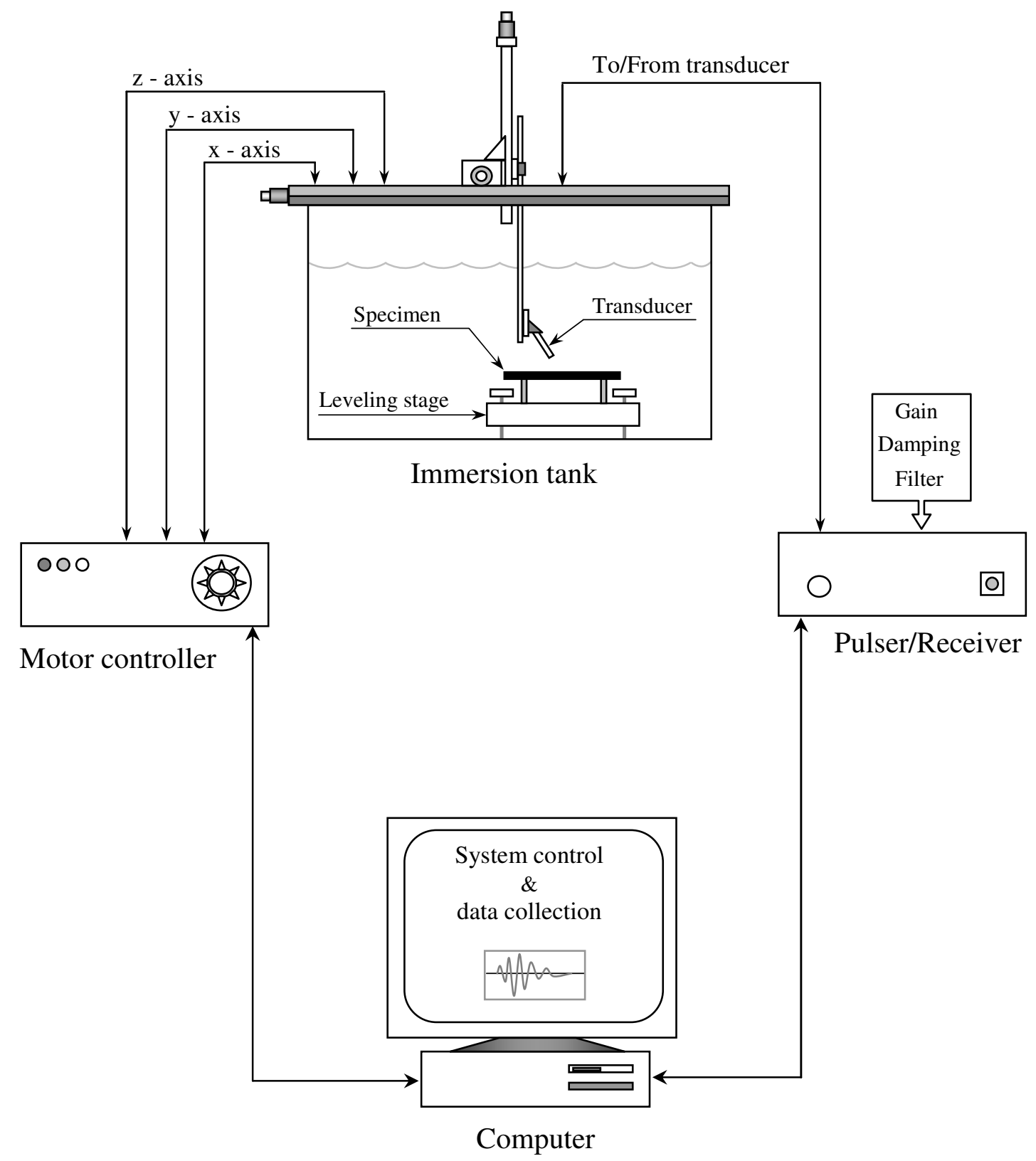

Fig. 2.7 A schematic representation of the ultrasonic scanning system. 
transducer along three mutually perpendicular axes in steps as small as $10 \mu \mathrm{m}$. The transducer is powered by a SONIX Pulser/Receiver (DPR35-S) system through which the input energy, gain, attenuation and high-pass filter can be controlled. The pulser/receiver allows the tests to be conducted in either the pulse-echo mode or the through-transmission mode. Water at room temperature was used as the couplant for all the tests.

Two broad-band, spherically focused transducers with center-frequencies of 20 $\mathrm{MHz}$ and $25 \mathrm{MHz}$ were used in this work. The $20 \mathrm{MHz}$ transducer had a $6.35 \mathrm{~mm}$ element diameter and $25.4 \mathrm{~mm}$ nominal focal length in water (Panametrics [26]), while the $25 \mathrm{MHz}$ transducer had an $8 \mathrm{~mm}$ element diameter and $12.5 \mathrm{~mm}$ nominal focal length in water. Frequency response for the $20 \mathrm{MHz}$ and $25 \mathrm{MHz}$ transducers was obtained by making the transducers normally incident on a thick aluminum specimen with a highly polished surface and taking the Fast Fourier Transform (FFT) of front surface reflection. The front surface reflection as obtained by the $20 \mathrm{MHz}$ and the 25 MHz transducers in the time domain is shown in Fig. 2.8(a) and Fig. 2.9(a) respectively, and the corresponding FFT for the two transducers is shown in Fig. 2.8(b) and Fig. 2.9(b) respectively.

A spherically focused transducer focuses the ultrasonic beam to a spot much smaller than the diameter of the transducer element, resulting in a much higher intensity of the acoustic beam near the region of the focal spot than at the transducer surface. A schematic illustrating the features of an acoustic beam from a focused ultrasonic transducer is shown in Fig. 2.10. To a first approximation, the acoustic beam converges 


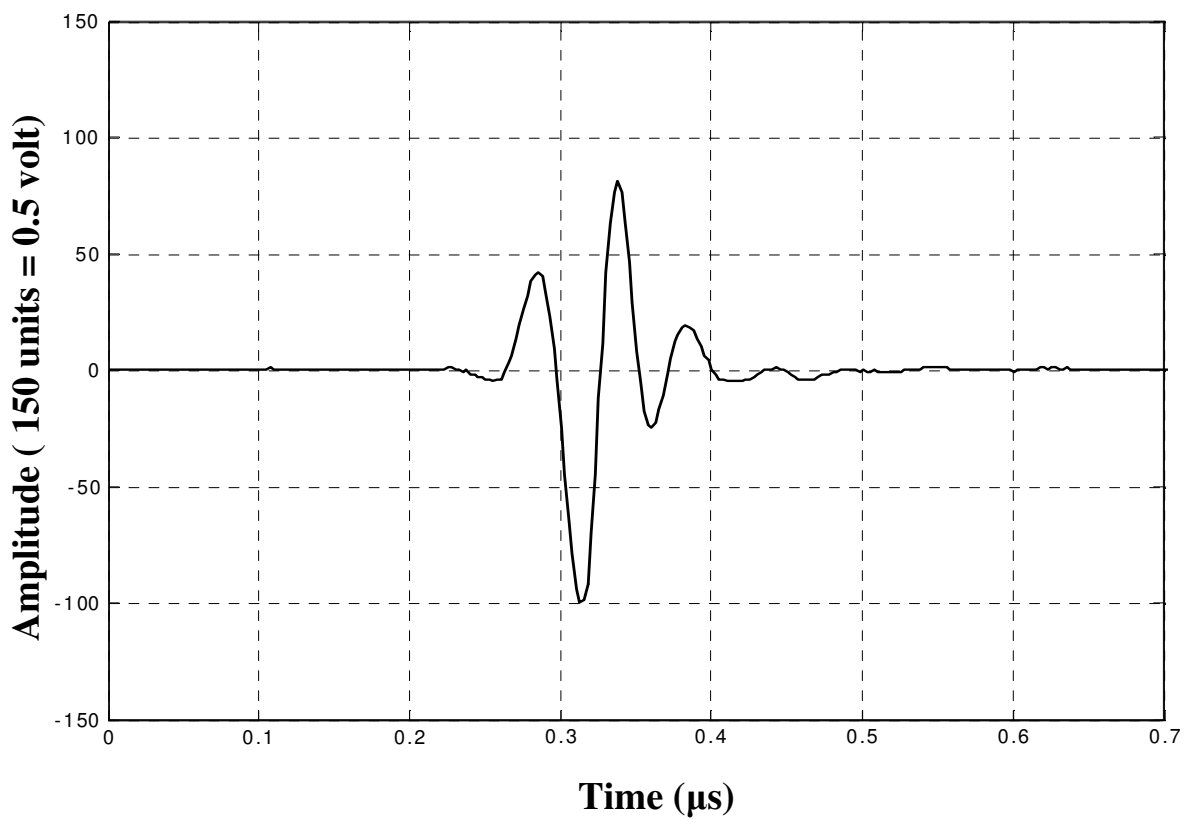

(a) Time domain

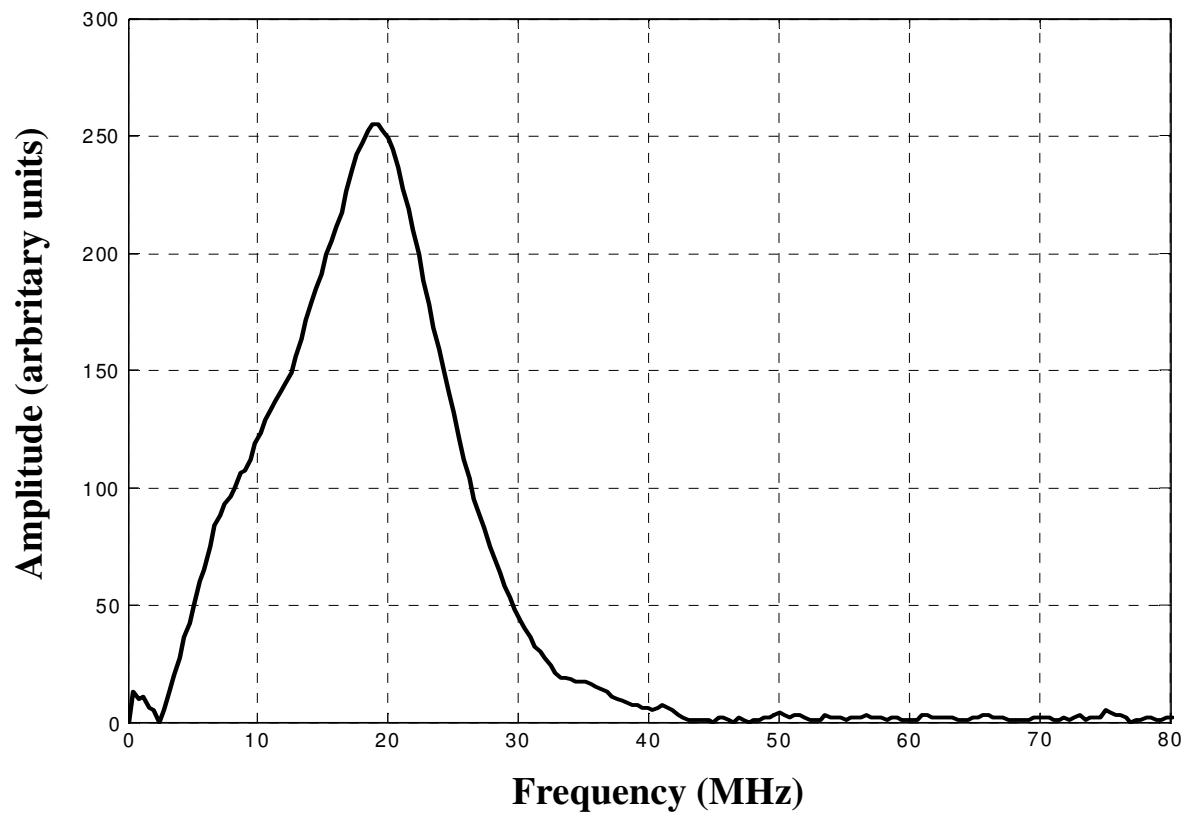

(b) Frequency domain

Fig. 2.8 Response of the $20 \mathrm{MHz}$ transducer;

(a) time domain, (b) frequency domain. 


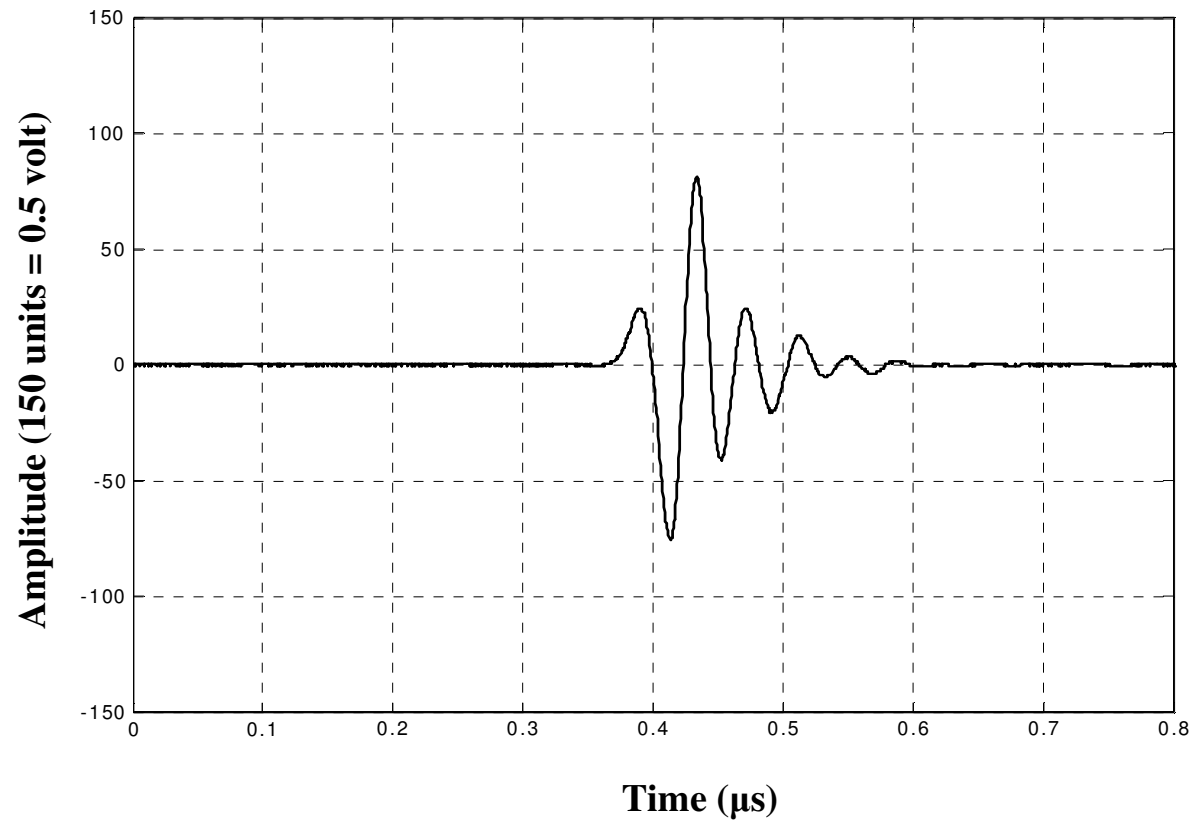

(a) Time domain

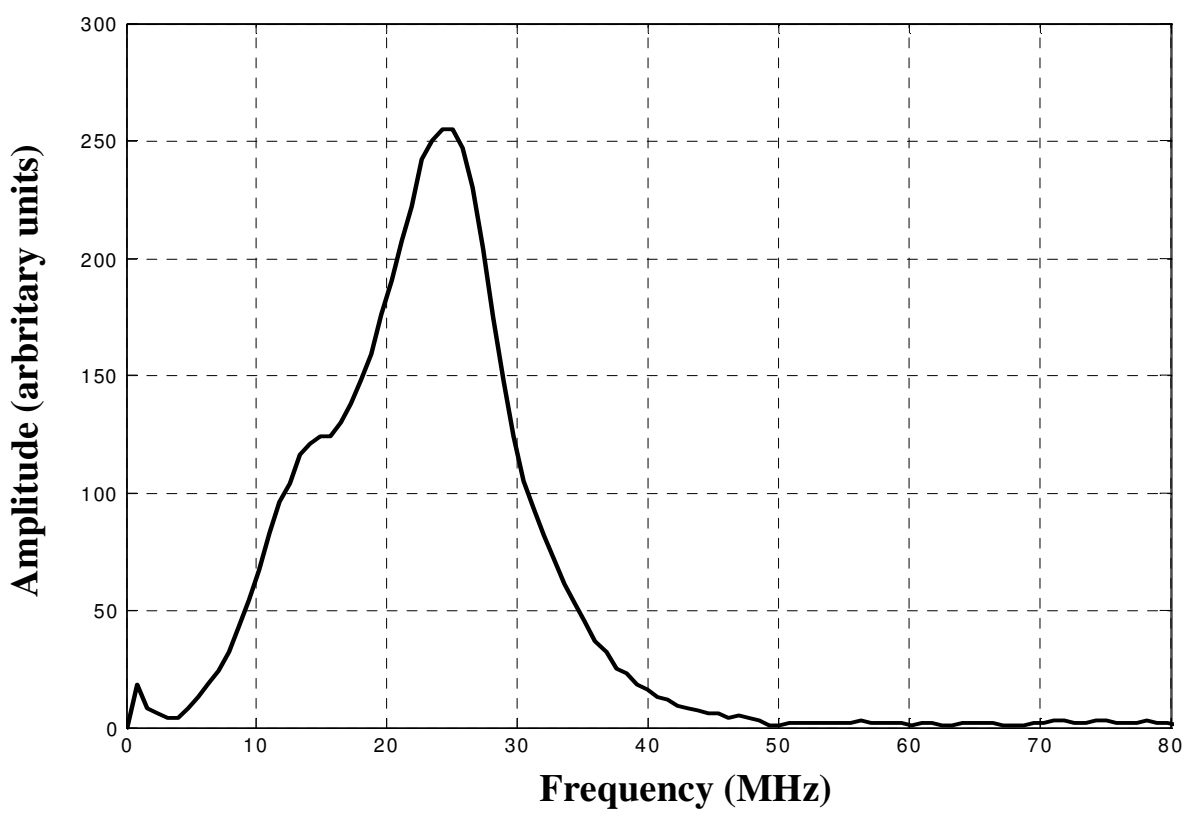

(b) Frequency domain

Fig. 2.9 Response of the $25 \mathrm{MHz}$ transducer; (a) time domain, (b) frequency domain. 
to a focal point and then diverges in accordance with geometrical optics (valid when $\lambda<<a$, where $\lambda$ is the wavelength and $a$ is a characteristic length, for example the radius of the transducer in present case). However in reality, as the acoustic beam nears the focal plane, instead of converging to a point it converges to a finite size spot. At the focal plane, the intensity is highest at the center of the beam and decreases in the radially outward direction. A detailed explanation on this topic is covered by Kino [27]. The diameter of the focal spot $(d)$ is defined as the diameter in the focal plane at which the intensity drops down by $3 \mathrm{~dB}$ from the peak intensity and is given by [27]

$$
d=\frac{0.51 \lambda F}{a}
$$

where $\lambda$ is the wavelength of longitudinal waves in the coupling medium, $F$ is the focal length of the transducer in the coupling medium and $a$ is the radius of the transducer element. As an illustrative example, for a $20 \mathrm{MHz}$ transducer with $3.18 \mathrm{~mm}$ element diameter and $25.4 \mathrm{~mm}$ nominal focal length the diameter of the focal spot in water $\left(\lambda_{\text {water }}=0.075 \mathrm{~mm}\right)$ is $0.31 \mathrm{~mm}$. Diameter of the focal spot directly affects the sensitivity and the spatial resolution of a transducer. For a given size of a flaw (less than the diameter of the focal spot) positioned at the focal spot, as the diameter of the focal spot decreases, the fraction of the energy reflected by the flaw increases leading to increased sensitivity. In the case of multiple flaws, for proper spatial resolution (the ability to discern between two distinct flaws), the diameter of the focal spot should be less than or equal to the spacing between the flaws.

The beam diameter also affects the smallest increment that can be set for 


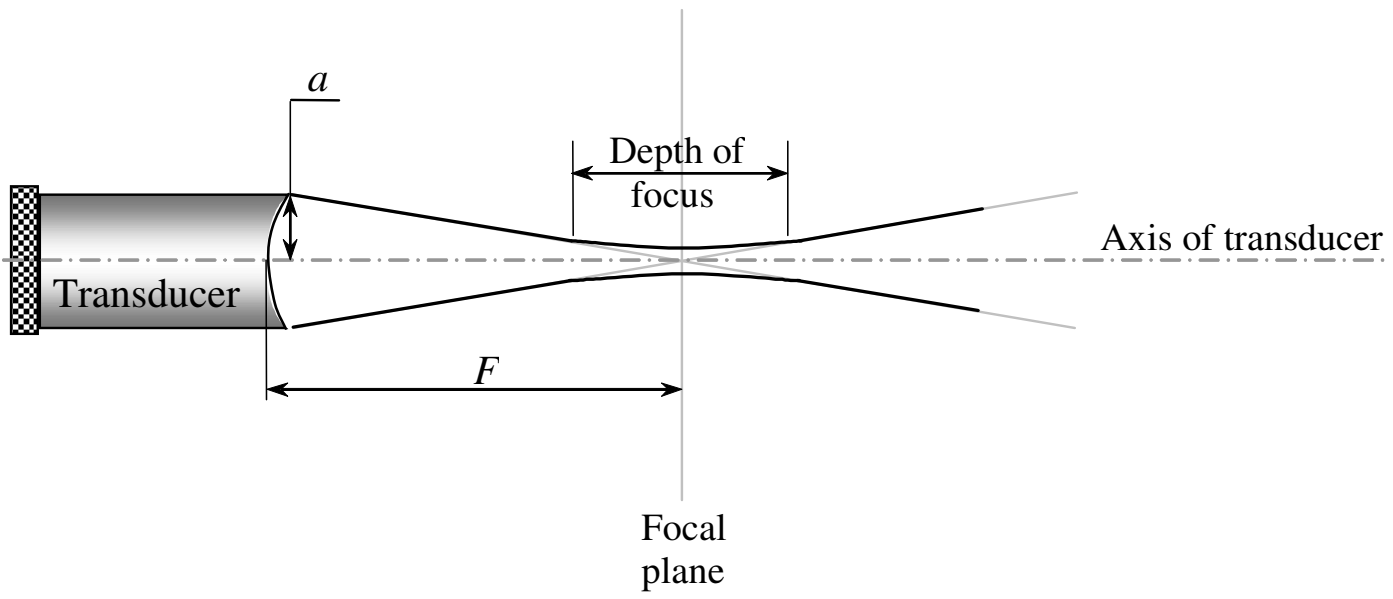

Fig. 2.10 Characteristics of the acoustic beam of a focused transducer. 
Table 3. Transducer Characteristics

\begin{tabular}{|l|l|l|}
\hline & $20 \mathrm{MHz}$ & $25 \mathrm{MHz}$ \\
\hline$\lambda_{\text {water }}(\mathrm{mm})$ & 0.075 & 0.06 \\
\hline$F(\mathrm{~mm})$ & 25.4 & 12.5 \\
\hline$a(\mathrm{~mm})$ & 3.18 & 3.18 \\
\hline$d(\mathrm{~mm})$ & 0.31 & 0.12 \\
\hline$l(\mathrm{~mm})$ & 8.64 & 1.73 \\
\hline
\end{tabular}


scanning the specimen for matrix cracks. In general the scanning step increment should be less than the diameter of the focal spot to ensure that the interrogation of the area between two consecutive scanning steps is not skipped by the transducer. In this thesis the scanning steps were set to $0.05 \mathrm{~mm}$, which is much less than the diameter of the focal spot for the $20 \mathrm{MHz}$ and the $25 \mathrm{MHz}$ transducers, namely $0.31 \mathrm{~mm}$ and $0.12 \mathrm{~mm}$, respectively.

The intensity of the ultrasonic beam also varies along the axis of the transducer. The distance between two points in the ultrasonic field along the axis where the intensity is $3 \mathrm{~dB}$ less than that at the focal point is called the 'depth of focus' [27]. Geometrically, the depth of focus may be interpreted as the distance between the points along the acoustic beam where it deviates from the expected geometrical optics (see Fig. 2.10). The specimen under investigation may be placed anywhere within the depth of focus. The dept of focus may be calculated as [27]:

$$
l=\frac{1.8 \lambda F^{2}}{a^{2}}
$$

The values of the two parameters described above were calculated for the two transducers, with water as coupling medium and are shown in the Table 3.

From Table 3 it is clear that the $25 \mathrm{MHz}$ transducer offers a smaller focal spot $(d)$ implying that damage may be detected with greater resolution than with the $20 \mathrm{MHz}$ transducer. On the other hand, the depth of focus $(l)$ is less than that for the $20 \mathrm{MHz}$ transducer implying lesser flexibility in adjusting the height of the transducer above the specimen. For most of the experiments, it was found that the sensitivity and spatial 
resolution provided by the $20 \mathrm{MHz}$ transducer were sufficient for the detection of matrix cracks. Therefore, in the following, the $20 \mathrm{MHz}$ transducer will be used unless otherwise stated.

\subsection{Testing Procedures}

\subsubsection{Ultrasonic Polar Backscattering}

The polar backscattering technique uses an obliquely incident ultrasonic beam as opposed to the conventional ultrasonic pulse-echo technique, which uses normally incident ultrasonic beam. The basic concept underlying the backscattering technique may be explained as follows. In the absence of a matrix crack the incident signal is reflected away from the transducer, which is configured to act as a transmitter as well as a receiver. This is illustrated in Fig. 2.11(a). For ease of understanding, the incident and reflected fields are collectively represented by arrows as shown in Figs. 2.11(a) and 2.11(b). The shaded area is a symbolic representation of the complicated interaction between the incident beam, the crack and the ply boundaries. When the crack is insonified by the incident beam, the incident signal interacts with the crack and is partly reflected back towards the transducer, as illustrated in Fig. 2.11(b).

A schematic showing the spatial arrangement of a transducer with respect to the orientation of a crack in a ply or a ply-group (i.e., adjacent plies of the same orientation) is shown in Fig. 2.12. An orthogonal coordinate system is chosen such that XYZ axes define the laminate (global) coordinate system. The fibers in the ply (and therefore the cracks) are oriented at an angle $\theta$ from the X-axis. For the incident beam, let $\alpha$ be the 


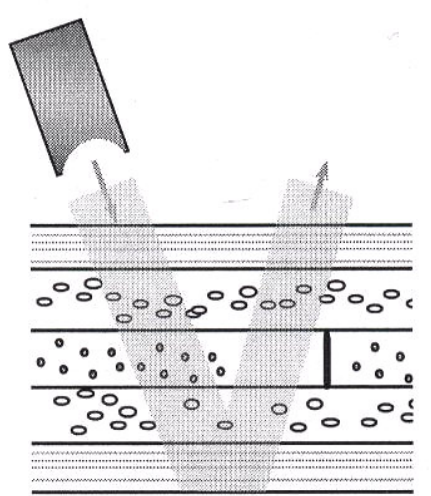

(a)

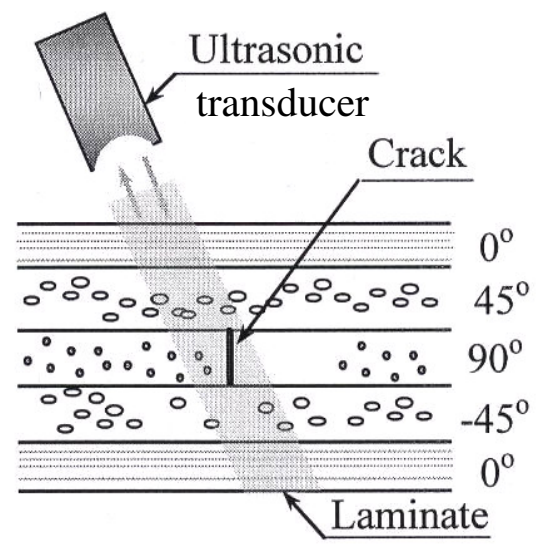

(b)

Fig. 2.11 A schematic of the concept underlying the ultrasonic polar backscattering technique. (All the incident and reflected waves are collectively represented by arrows pointing into and out of the specimen respectively. The shaded area is a symbolic representation of the complicated interaction between the incident beam, the crack and the ply boundaries.) 


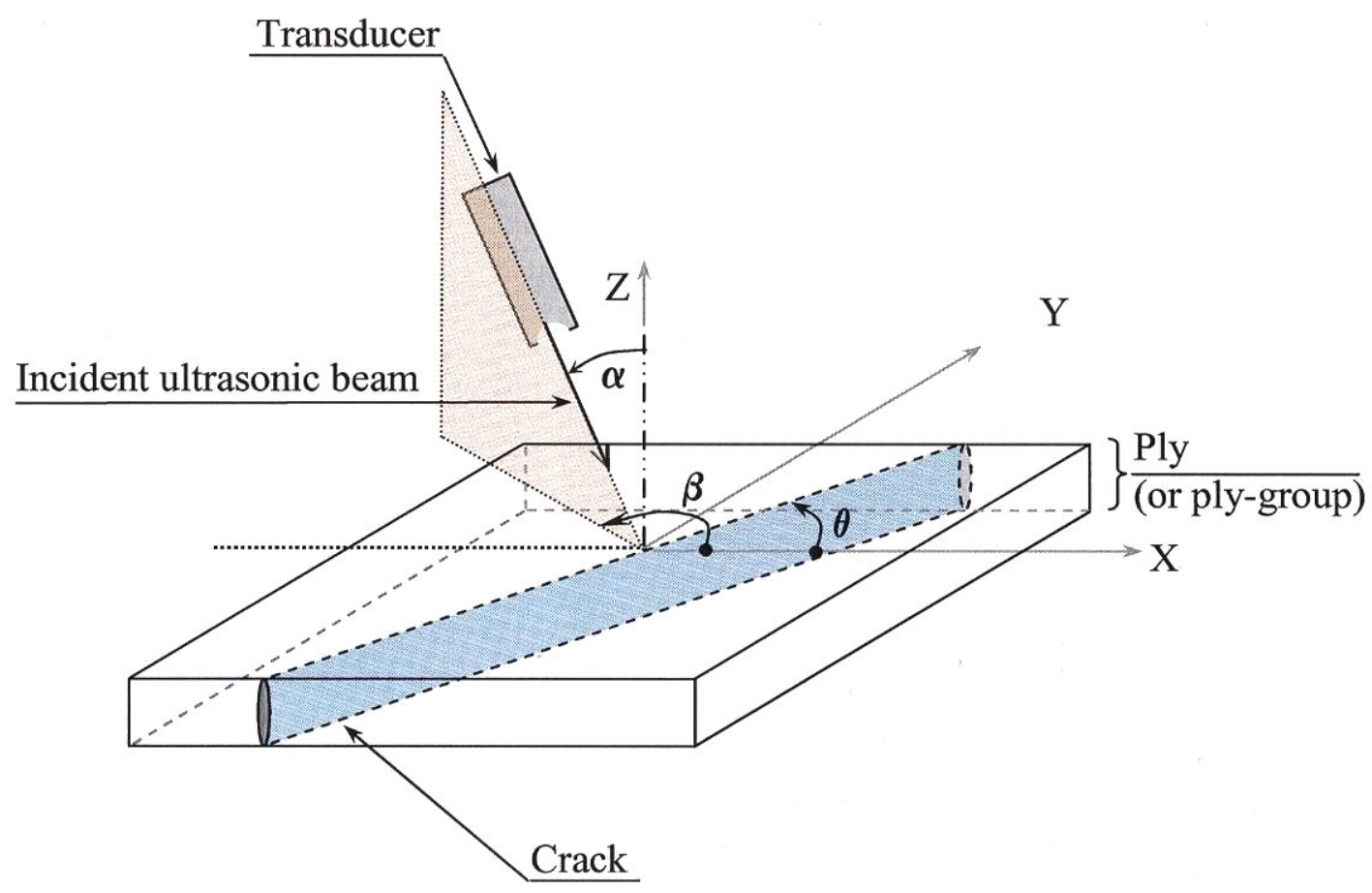

Fig. 2.12 A schematic showing a general spatial arrangement of the ultrasonic transducer with respect to a cracked ply or ply-group. 
angle of incidence as measured from the normal and let $\beta$ be the azimuthal angle measured from the X-axis, positive in the counter-clockwise direction. To determine the angle $\beta$ at which the received signal is maximum, a small area of a specimen containing cracks was initially scanned using a transducer adjusted such that the projection of incident ray onto the laminate is perpendicular to the crack faces (i.e. $\beta=\theta \pm \pi / 2$ ). Then, from this position, for a fixed angle of incidence and fixed height above the specimen, the transducer was rotated such that value $\beta$ varied a few degrees back and forth from the initial adjustment. At each value of angle $\beta$, the same area was scanned again and the amplitude of the received signal from a particular crack was plotted. One such plot for a crack in the third ply $\left(\theta=90^{\circ}\right)$ of the 18-ply TAMU-5 specimen is shown in Fig. 2.13. A schematic of the plan view of the experimental arrangement is shown above the plot. The azimuthal angle $\beta$ was initially set at $180^{\circ}$ and varied over a range of $24^{\circ}\left( \pm 12^{\circ}\right)$ from the initial value, in steps of $2^{\circ}$. The relative amplitude on the $y$-axis is in decibels and is calculated as

$$
\text { Relative amplitude }=20 \log _{10}\left(\frac{V}{V_{\max }}\right)
$$

where $V$ is the corresponding absolute amplitude in volt and $V_{\max }$ is the maximum amplitude in volt in a given set of amplitude data. From Fig. 2.13 it is clear that the amplitude of the reflected signal is maximum when the projection of incident ray onto the laminate is perpendicular to the crack faces, i.e. when $\beta=180^{\circ}$. This fact was used to generate ultrasonic images of cracks in different plies of a composite laminates. For example, for a simple case of a [0/90] laminate shown in Fig. 2.14, a crack in the first 

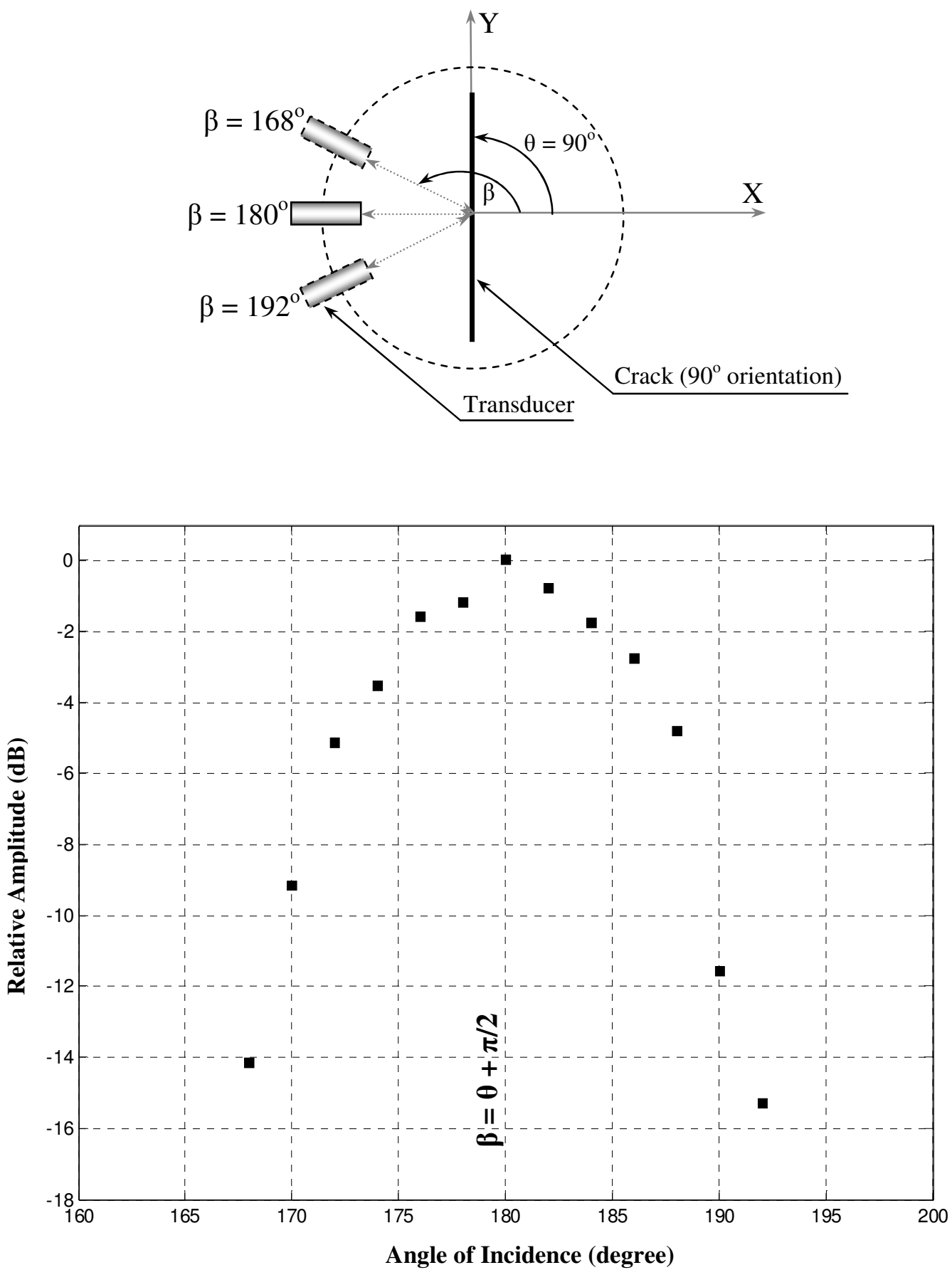

Fig. 2.13 Variation in the amplitude of the reflected signal with changes in the azimuthal angle $(\beta)$. 
ply $\left(\theta=0^{\circ}\right)$ can be detected by setting the azimuthal angle $\beta=90^{\circ}$ (Fig. 2.14(a)). Similarly a crack in the second ply $\left(\theta=90^{\circ}\right.$ ) can be detected by setting $\beta=180^{\circ}$ (refer Fig. 2.14(b)).

The angle of incidence $\alpha$ was adjusted such that the amplitude of the signal reflected from the crack, as detected by the transducer, is maximum. To determine this angle experimentally, a small portion of a specimen containing cracks was scanned repeatedly at various angles of incidence. The azimuthal angle $\beta$ was fixed as explained earlier. For each angle, the height of the transducer was adjusted in such a way the focal spot of the transducer was always coincident with the top surface of the specimen. This ensured that the variations in the reflected signal due to the defocusing the incident beam is avoided. At each angle, the peak amplitude (in volt) of the signal received from a representative crack is plotted; the angle corresponding to the maximum amplitude in this plot is chosen as the angle of incidence. Figure 2.15 shows a plot of relative peak amplitudes of the signal received observed at the corresponding angles of incidence, for a crack in the third ply $\left(90^{\circ}\right.$ orientation) of the 18-ply TAMU-5 specimen. The relative amplitude was calculated as explained earlier. From Fig. 2.15 it is clear that the amplitude of the reflected signal is highest at about $25^{\circ}$ angle of incidence. However, it may be noted that the amplitude is within $6 \mathrm{~dB}$ of the highest amplitude for angles of incidence between $20^{\circ}$ and $30^{\circ}$, instructive of the flexibility possible in adjusting the angle of incidence. In this work, all the experiments were conducted with angle of incidence $\alpha$ equal to $25^{\circ}$ unless mentioned otherwise.

The height of the transducer above the specimen had to be fixed experimentally 

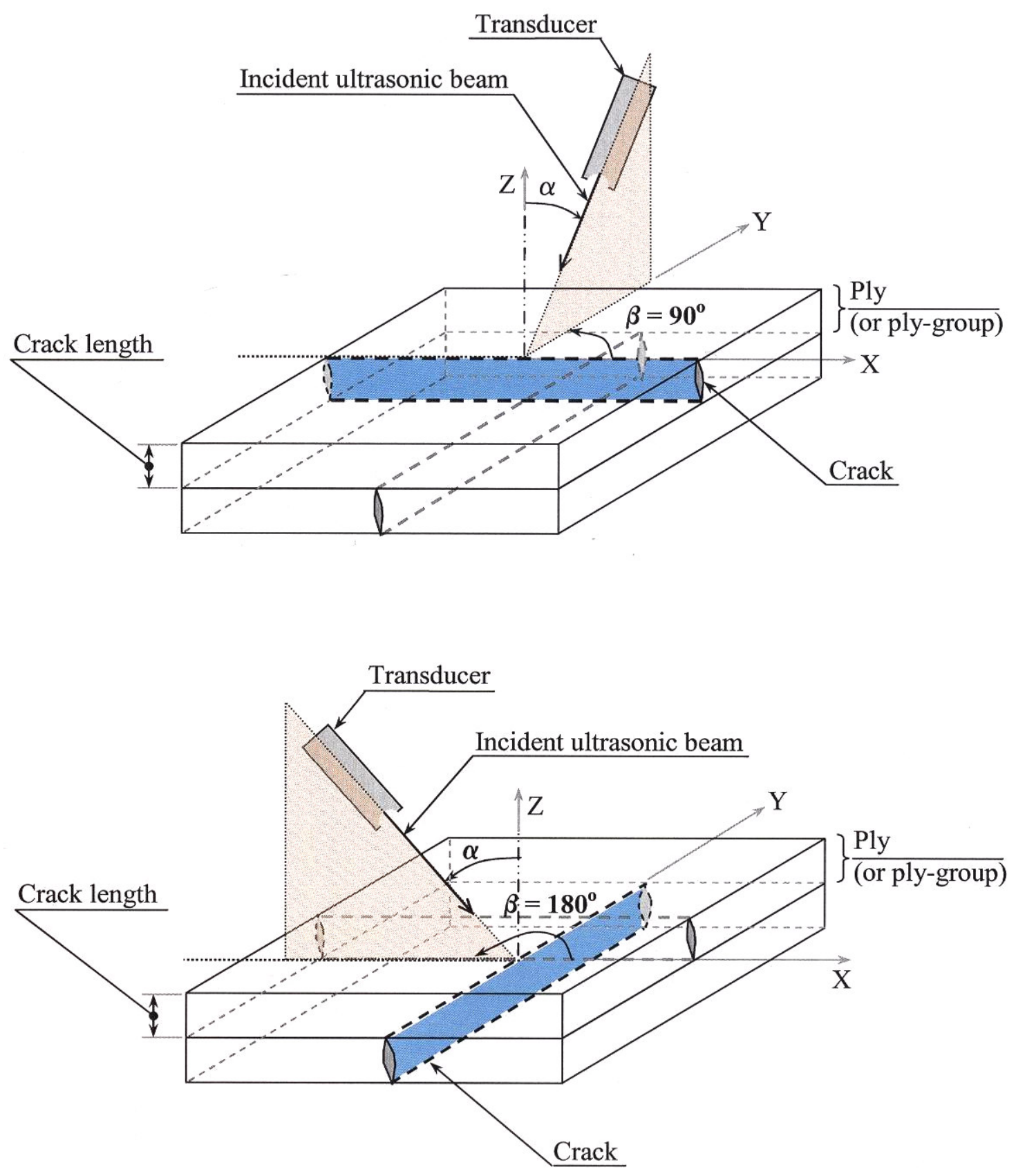

Fig. 2.14 A schematic of the spatial arrangement of the ultrasonic transducer with respect to a cracked ply or ply group. (a) To detect a crack in the first ply $\left(0^{\circ}\right),(\mathrm{b})$ to detect a crack in the second ply $\left(90^{\circ}\right)$. 


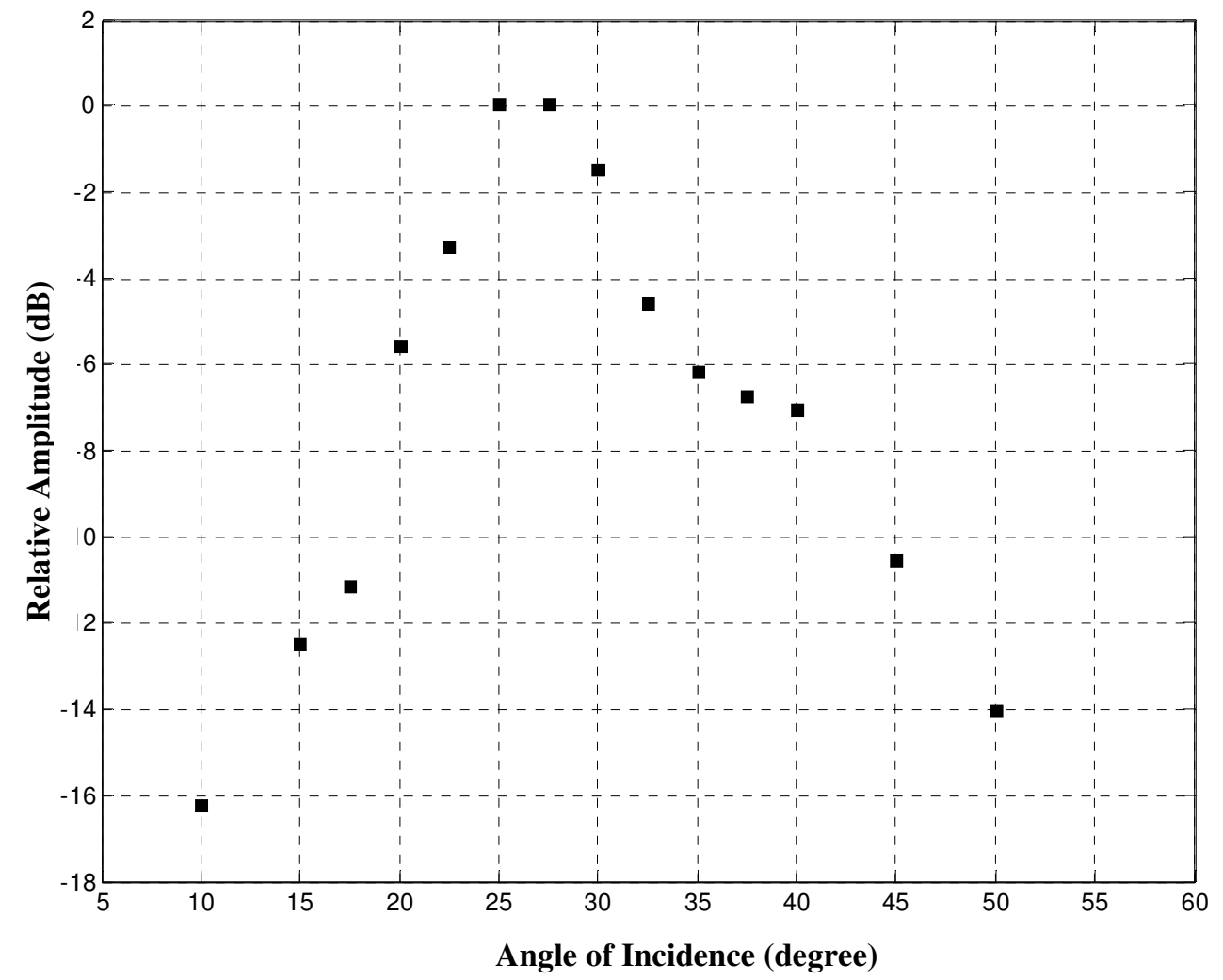

Fig. 2.15 Variation in the amplitude of the reflected signal with changes in the angle of incidence $(\alpha)$. 
since no data is available to the author's knowledge that will allow theoretical calculation of the change in the focal length when an acoustic beam travels from an isotropic medium to anisotropic medium. This was done by varying the vertical distance between the center of the transducer and the top of the specimen in steps of $1 \mathrm{~mm}$, at a fixed angle of incidence $\left(25^{\circ}\right.$ in the present case). At each step, a small area of a specimen containing matrix cracks was scanned and the peak amplitude from a selected crack is recorded. Figure 2.16 shows the variation of the peak amplitude with change in vertical distance between the transducer and the specimen surface. It can be observed that the strength of the reflected amplitude is maximum when the transducer is arranged such that the focal spot is located around the upper surface of the specimen.

In the case where there are two non-contiguous plies (or ply-groups) of the same orientation, each will reflect the incident wave back to the transducer. However, the signal from the deeper ply (or ply-group) will arrive with a certain time delay after the arrival of the signal from the shallower ply (or ply-group). Hence, the images of the cracks in the two plies (or ply-groups) can be obtained by resolving the two signals with the help of appropriate time-gates.

A typical time domain signal in the absence of a crack is shown in Fig. 2.17(a). It is seen from Fig. 2.17(a) that the signal received in the absence of a crack is not zero but some small value. This is most likely due to the backscattering of the incident ultrasonic beam by the fibers and/or fiber-bundles [20]. For the same laminate, a typical time domain signal generated by the reflected ultrasonic waves when the incident ultrasonic beam insonifies a matrix crack is shown in Fig. 2.17(b). The signal corresponds to a 


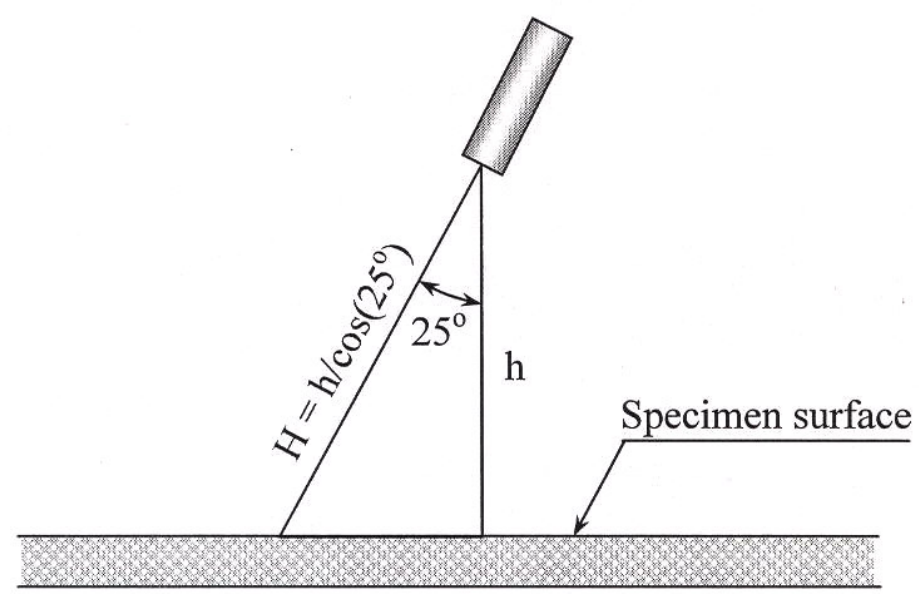

Axial distance: $\mathbf{H}(\mathbf{m m})$

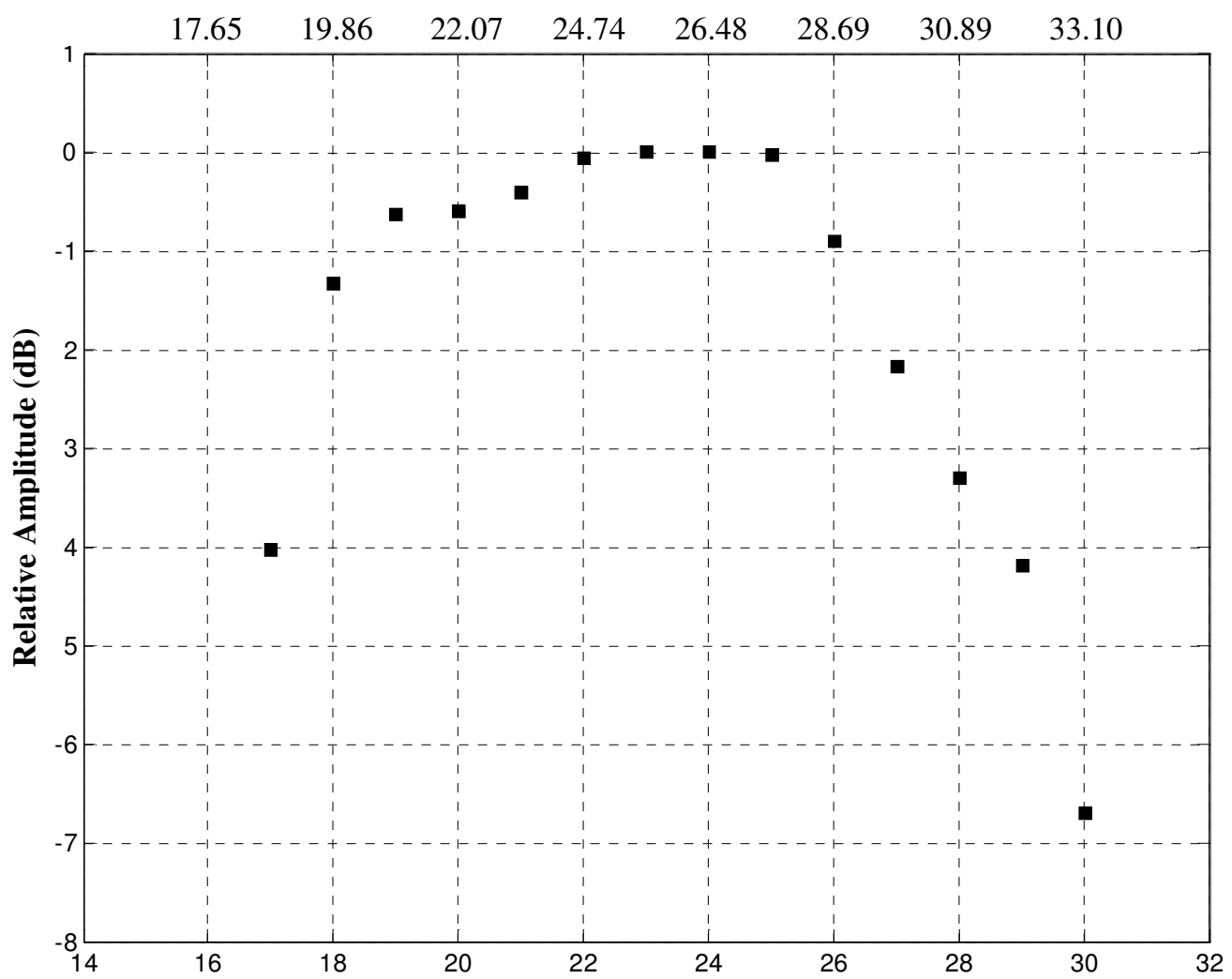

Vertical Distance Between the Transducer and the Top Surface of the Specimen: $\mathbf{h}(\mathrm{mm})$

Fig. 2.16 Variation in the amplitude of the reflected signal with changes in the vertical distance between the transducer and the specimen. (Angle of incidence: $25^{\circ}$ ) 


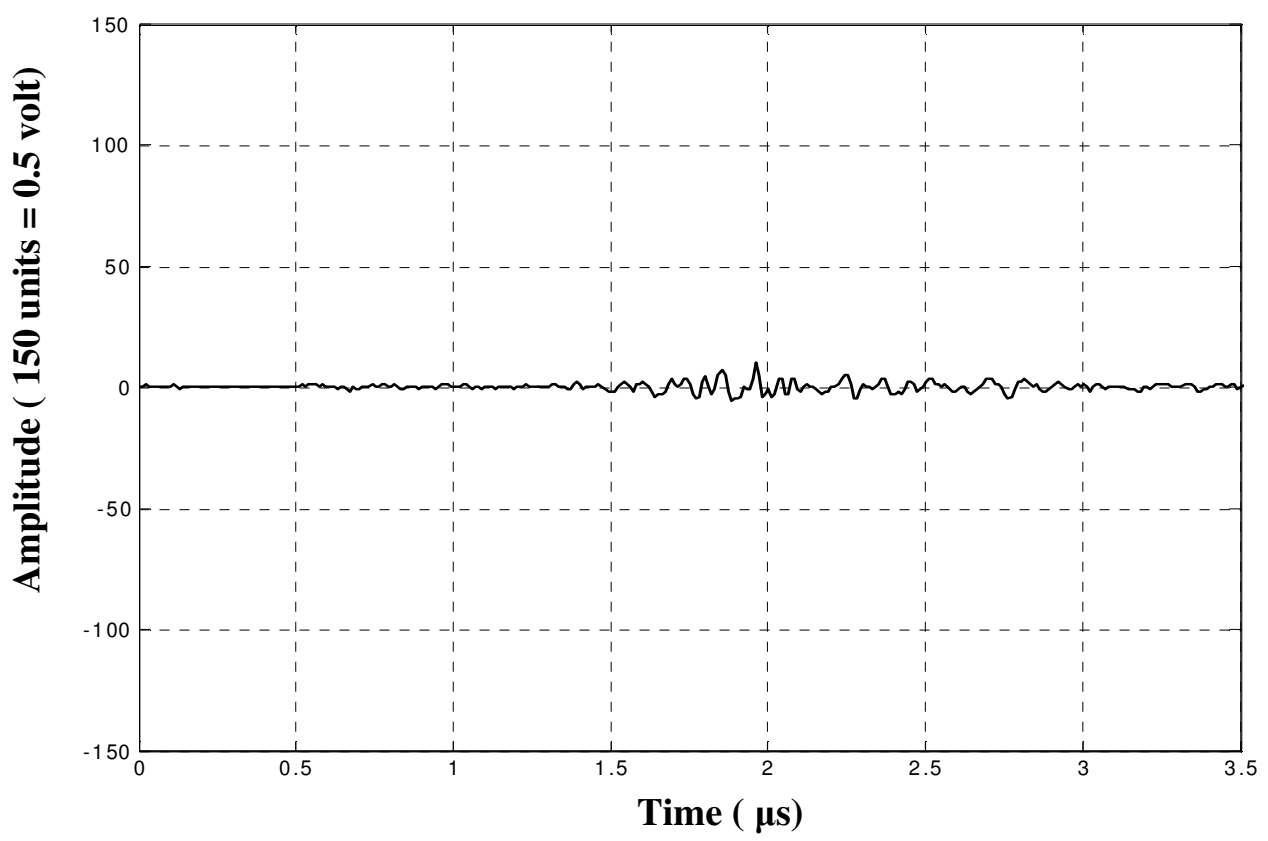

(a) Typical time domain signal in the absence of a crack.

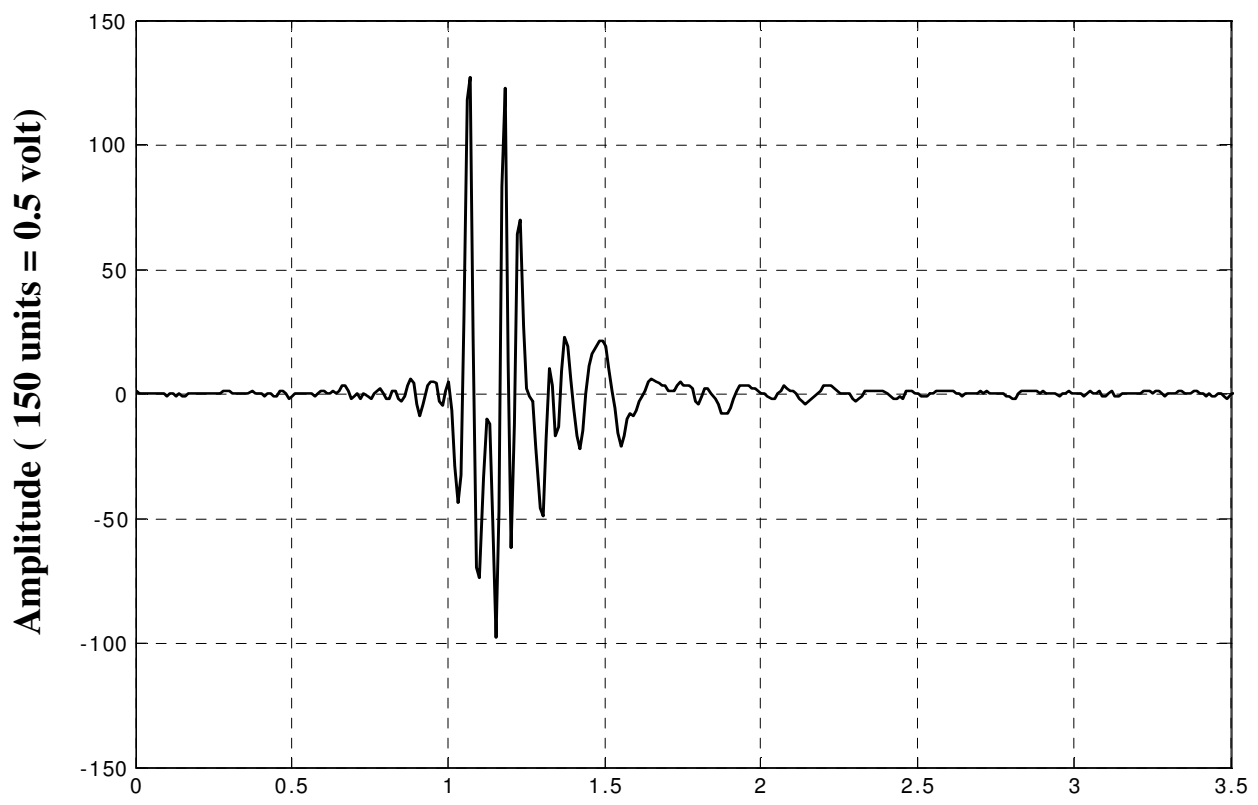

Time ( $\mu s)$

(b) Typical time domain signal in the presence of a crack.

Fig. 2.17 Typical time domain signals observed in case of the TAMU-5 specimen using the polar backscattering technique. 
crack in the third ply $\left(90^{\circ}\right.$ orientation) of the 18-ply TAMU-5 laminate. The backscattered signal by the fibers (seen in Fig. 2.17(a)) is the main contributor towards the noise in the system. However, in most cases reported in this work, the backscattering noise is very weak and the signal to noise ratio was usually found to be above $20 \mathrm{~dB}$ indicating a very high level of confidence.

\subsubsection{Calibration Techniques}

In order to ensure that the received signal is due to a reflected field caused by the presence of crack, a few canonical experiments were conducted. These will be described in the following. In the first experiment, a pair of matched focused transducers (A and B: $20 \mathrm{MHz}$ center-frequency and $25.4 \mathrm{~mm}$ (1") focal length) was used. As illustrated in Fig. 2.18, when $\mathrm{A}$ acts as a transmitter and $\mathrm{B}$ as a receiver, the arrangement is referred to as the 'pitch-catch' method. Setting the transducer A to act as a transmitter as well as a receiver gives the polar backscattering arrangement. A small area of the 10 -ply $\left[0 / 90_{4}\right]_{\mathrm{s}}$ CALB-1 laminate was scanned.

In the absence of a crack, the reflected signal is detected by the receiver B as illustrated in Fig. 2.18(a). However, when a crack appears in the 'line-of-sight' it prevents the reflected signal from reaching the receiver B as shown in Fig. 2.18(b). Consequently, a crack should show up as a dark band on a bright background. A typical time-domain representation of the received signals in the absence of crack is shown in Fig. 2.19(a) and that in the presence of a crack is shown in Fig. 2.19(b). As mentioned earlier, in the schematics shown in Figs. 2.19(a) and 2.19(b), the incident and 


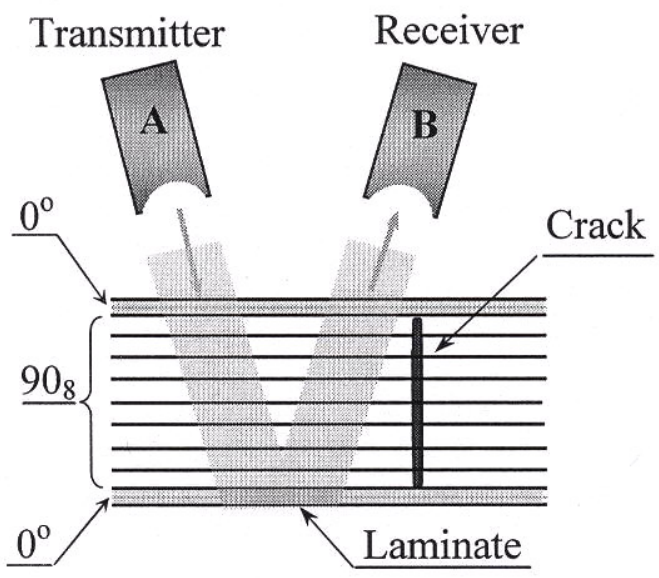

(a)

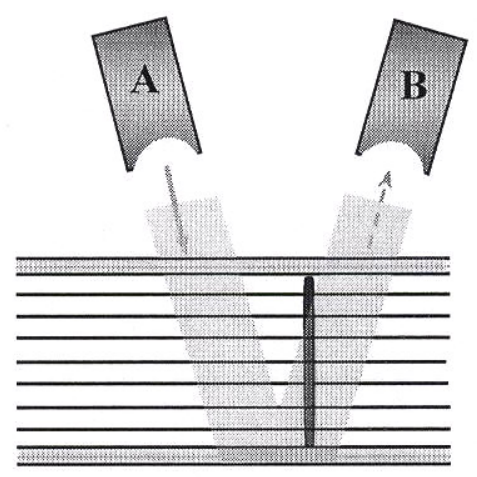

(b)

Fig. 2.18 A schematic of the experimental setup for the ultrasonic pitch-catch method. (a) In the absence of a crack, transducer B receives the reflected signal, (b) crack blocks the signal from reaching transducer B. 
the reflected fields are collectively represented by arrows and the shaded area is a symbolic representation of the complicated interaction between the incident beam, the crack and the laminate. As expected, the front surface reflection in the presence of crack (Fig. 2.19(b)) does not show a large variation when compared to that in the absence of crack (Fig. 2.19(a)); whereas, in the presence of a crack, a drastic reduction in the amplitude of the back surface reflection is observed. A 400 ns gate was used to record the changes in the amplitude of the back surface reflection as shown in Figs. 2.19(a) and 2.19(b) and the resulting ultrasonic images are shown in Fig. 2.20(a). The dark bands, as expected, correspond to the cracks. At the same time, in the polar backscattering method, with transducer A acting as the transmitter as well as the receiver, one should expect the cracks to appear as bright bands on a dark background. The results are shown in Fig. 2.20(b). Here, the bright bands, as expected, correspond to the cracks. One should not expect to see a sharp line corresponding to a sharp crack because these ultrasonic images are not optical images of a crack in the sense of photography. Instead, these are grayscale representation of acoustic field received after a rather complex interaction with the crack. A one-to-one correspondence between the images of cracks obtained by using pitch-catch (Fig. 2.20(a)) and polar backscattering (Fig. 2.20(b)) is observed. As an independent corroboration, the edges of the specimen were observed under a microscope at $200 \mathrm{X}$ magnification. A schematic of the optically detected cracks (Fig. 2.20(c)) is included at the bottom of the Fig. 2.20(b). It is reassuring to note that there is a one-toone correspondence between the optical data and the ultrasonic data. 


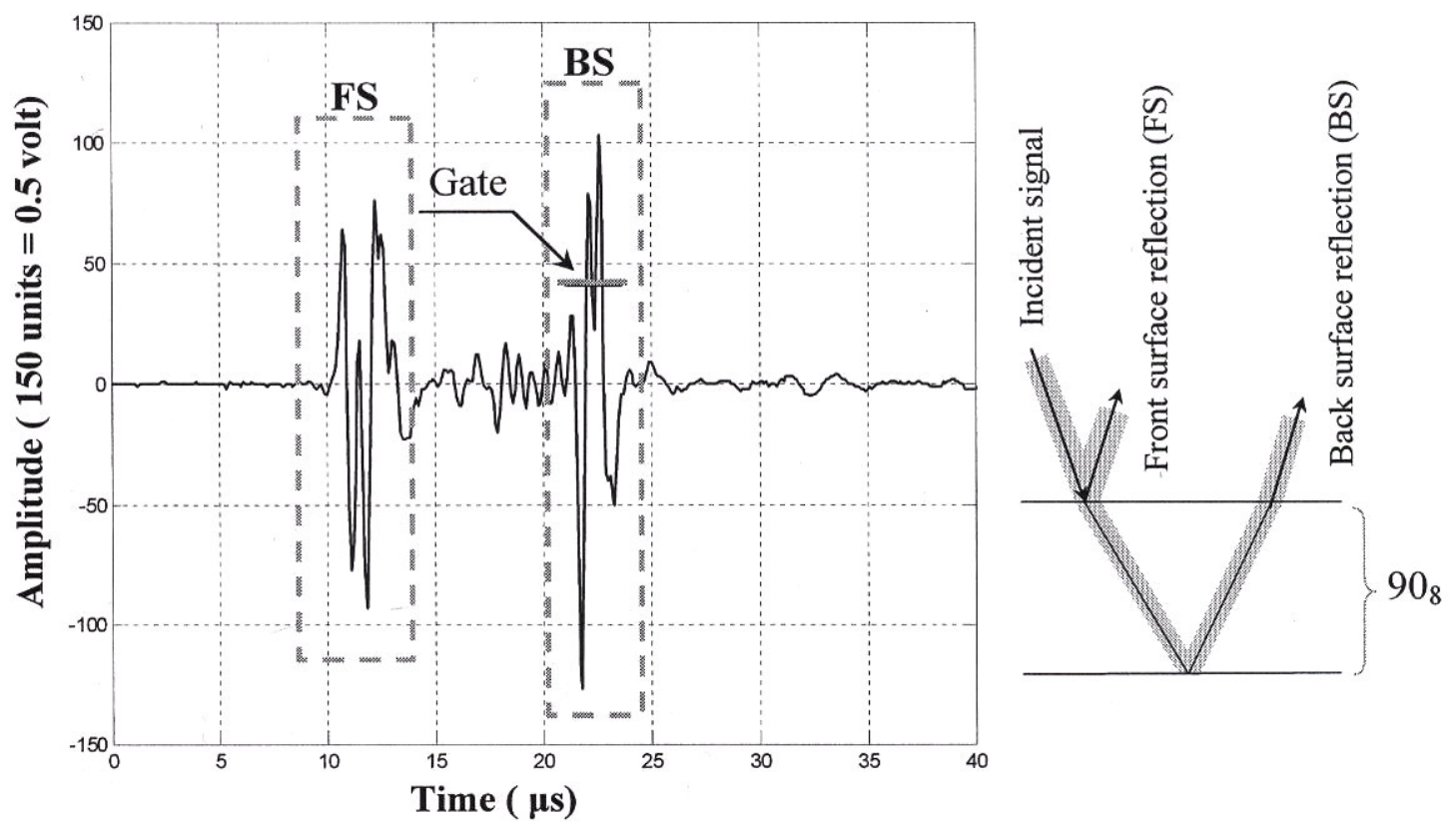

(a) Typical time domain signal in the absence of a crack

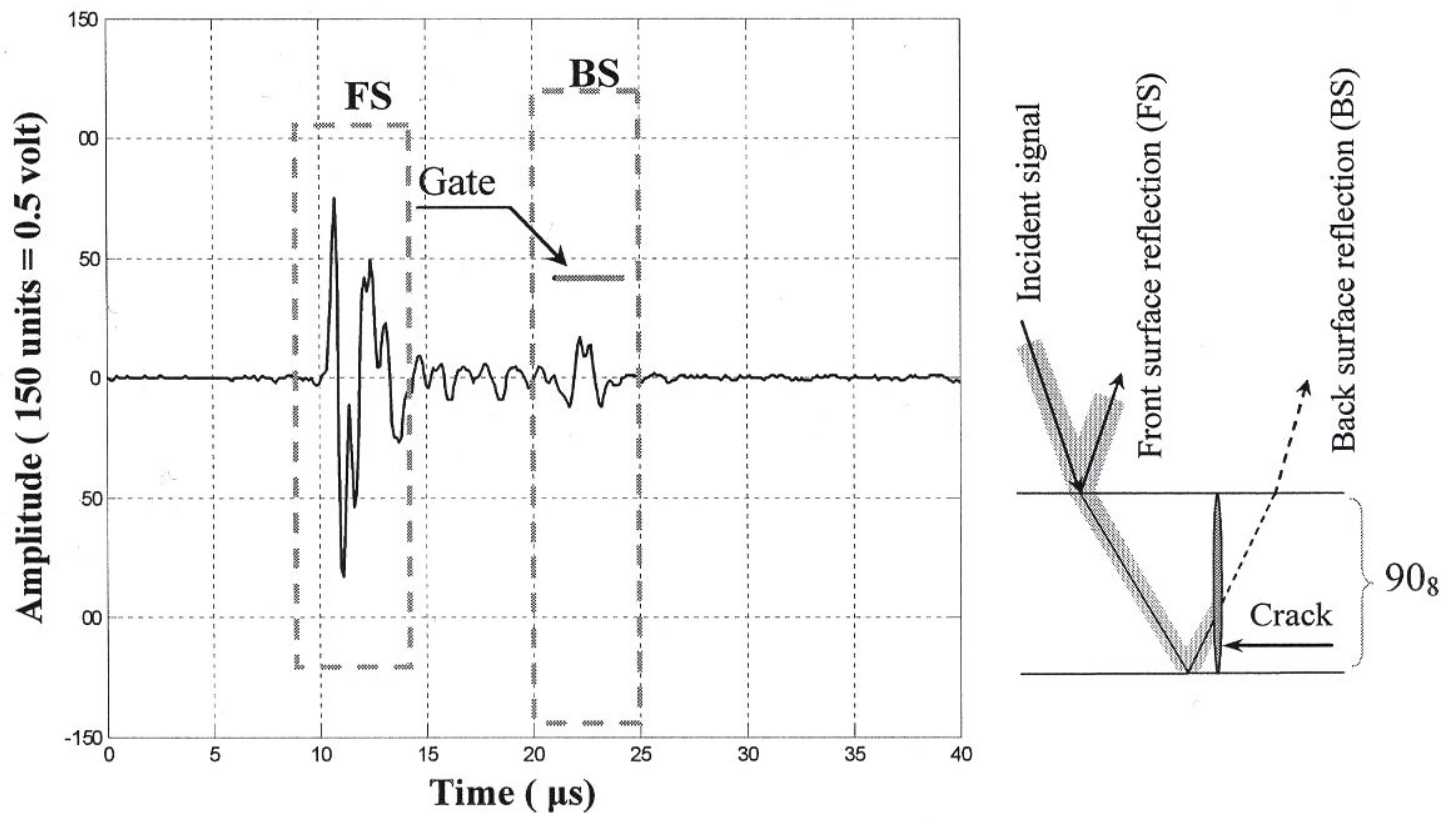

(b) Typical time domain signal in the presence of a crack

Fig. 2.19 Typical time domain signals observed in case of the CALB-1 specimen using the pitch-catch technique shown in Fig. 2.16. The transducer is oriented to detect cracks in the $90_{8}$ ply-group. 
(a)

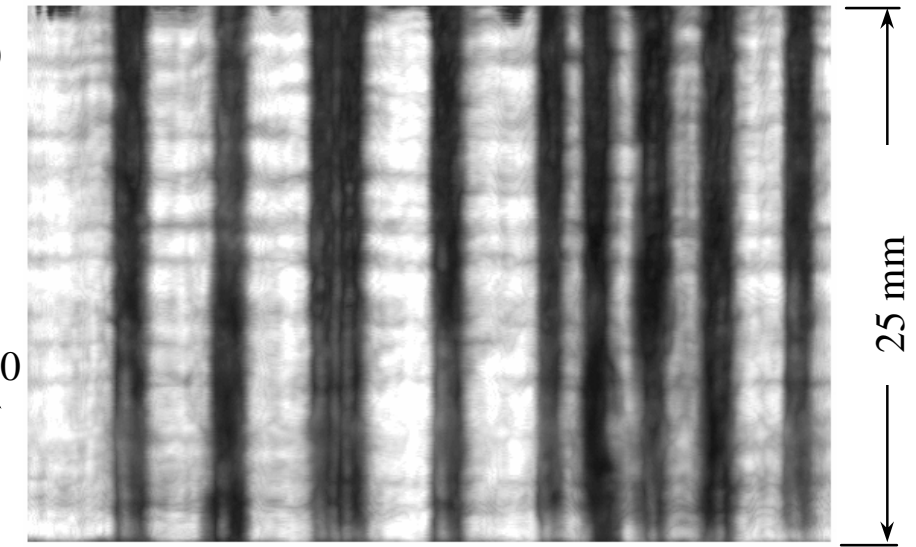

(b)

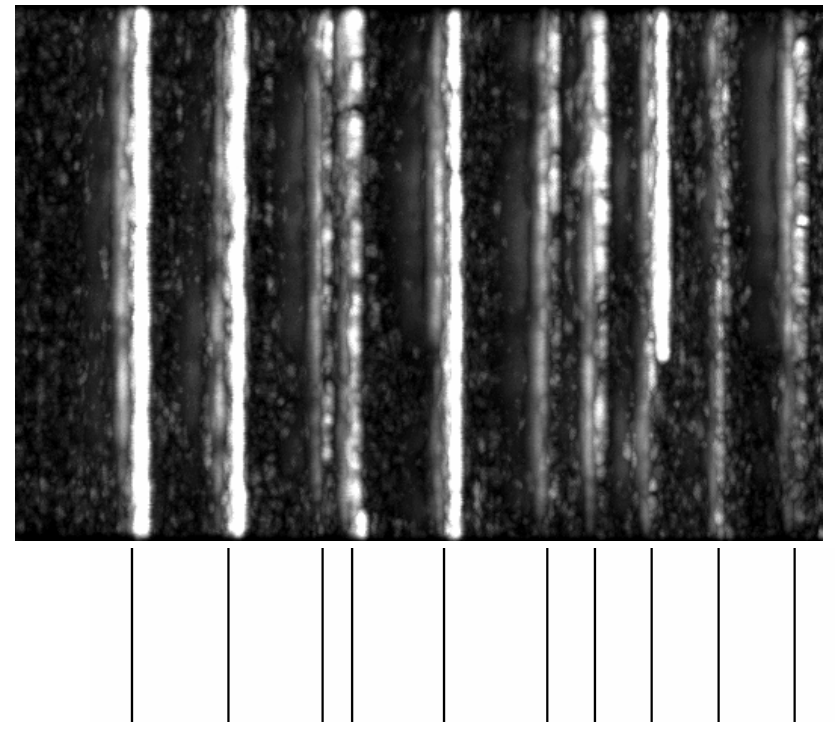

(c)

Fig. 2.20 Ultrasonic images of matrix cracks in the $\left[0 / 90_{4}\right]_{\mathrm{s}}$ CALB-1 laminate. (a) pitch-catch method, (b) polar backscattering, (c) schematic of the optically detected cracks at the edge of the specimen. 
In order to guard against fortuitous results, the preceding experiments were repeated on the 8-ply $\left[0 / 90_{3}\right]_{\mathrm{s}}$ CALB-2 specimen. The laminate was scanned for cracks in the $90_{6}$ ply-group. The results obtained with the pitch-catch and the polar backscattering methods are shown in Figs. 2.21(a) and 2.21(b). A schematic of the optically detected cracks is shown in Fig. 2.21(c). Once again, it is reassuring to obtain a one-to-one correspondence between the two methods for the CALB-2 laminate.

It is well known that wave propagation in laminated composites is an extremely complex phenomenon [28-30]. Nevertheless, some attempts were made theoretically model the experimental results reported in Fig. 2.20; these attempts were largely unsuccessful. The knowledge gained during the exercise is documented in the appendix for future use.

If the received signal is indeed a specularly reflected field, then it should follow the basic law of reflection, i.e. the angle of reflection must be equal to the angle of incidence. The second experiment was conducted to ascertain if the received signal indeed followed this law. The same pair of matched focused transducers was arranged in such a way that the crack acts as a reflector of the ultrasonic signal from one transducer towards the other transducer. This is arrangement is represented schematically in Fig. 2.22. Transducer $\mathrm{A}$ is set as the transmitter, while transducer $\mathrm{B}$ is set as the receiver. Both the transducers are inclined at the same angle $\alpha$ to the normal and their position is symmetric with respect to the $\mathrm{X}$-axis (i.e. $\beta_{1}=\beta_{2}$ ) as seen in Fig. 2.22. The transducers are arranged in such a way as to share the same focal spot (con-focal arrangement). In the absence of a crack, there is no mechanism by which the incident signal may be 
(a)

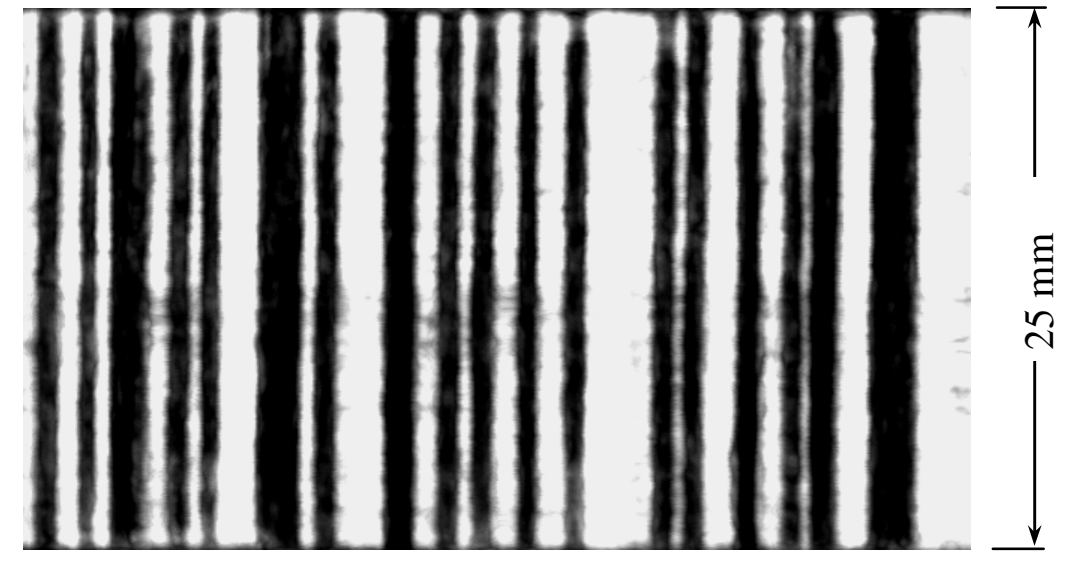

(b)

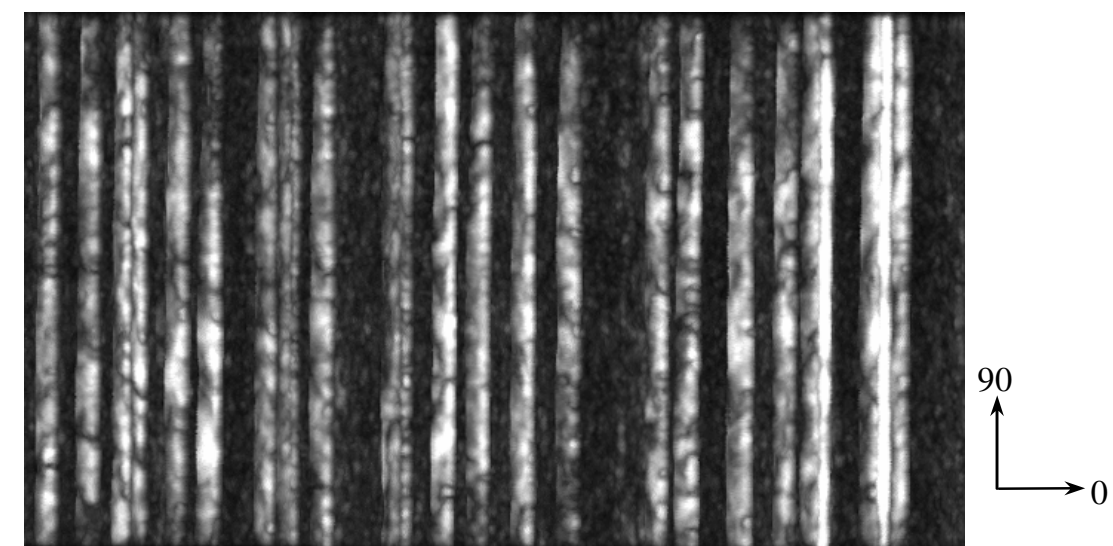

(c)

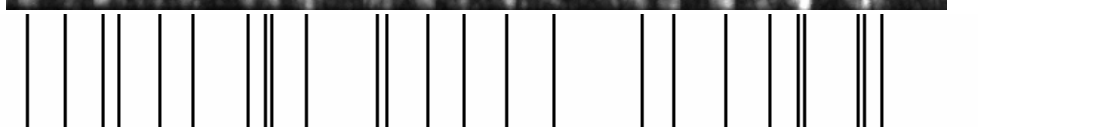

Fig. 2.21 Ultrasonic images of matrix cracks in the $\left[0 / 90_{3}\right]_{\mathrm{s}}$ CALB-2 laminate. (a) pitch-catch method, (b) polar backscattering, (c) schematic of the optically detected cracks at the edge of the specimen. 


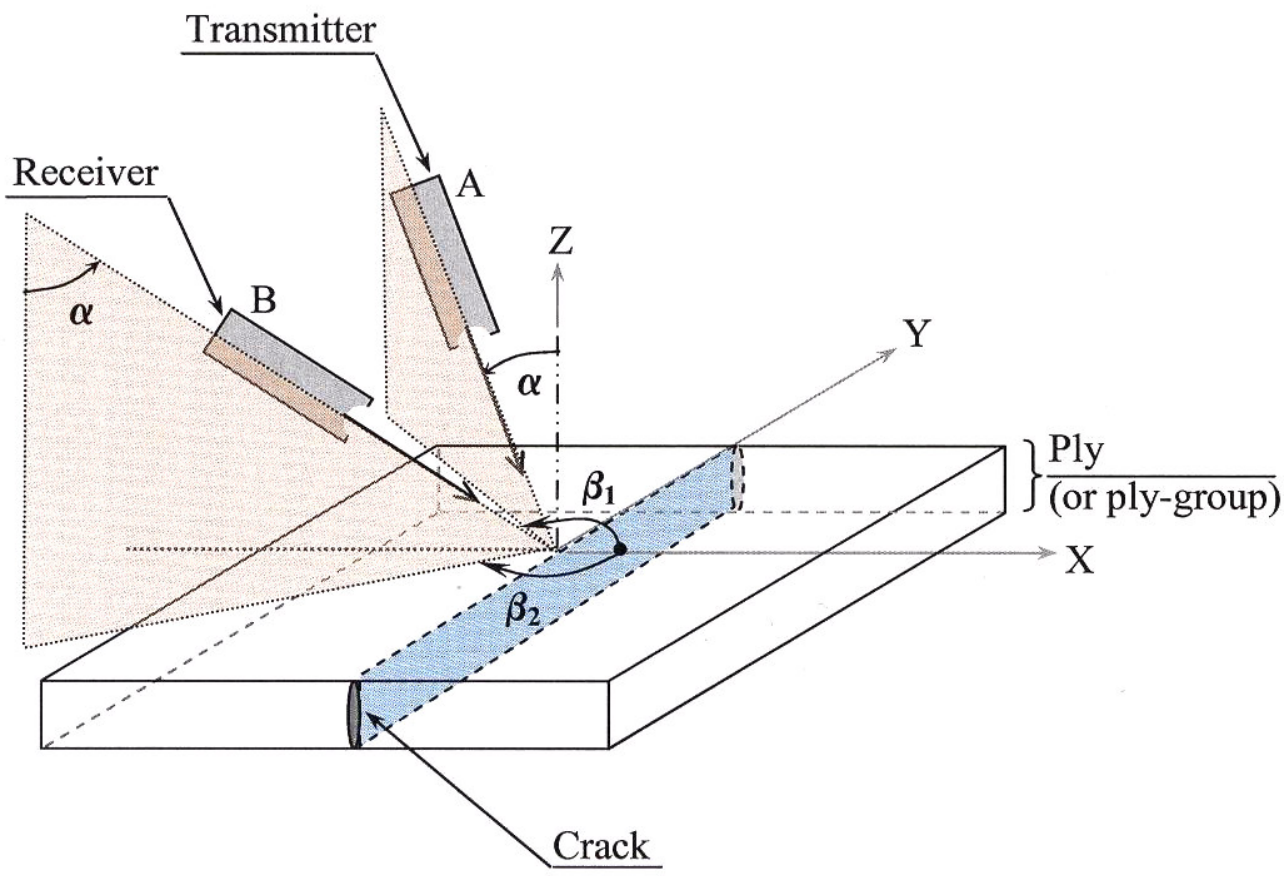

Fig.2.22 A schematic showing the arrangement of the transducers A and B in order that the signal from transducer $\mathrm{A}$ is reflected towards transducer $\mathrm{B}$. (The condition that $\beta_{1}=\beta_{2}$ is necessary for transducer $B$ to detect the reflected signal.) 
reflected towards the receiver. Consequently, the regions without any cracks should appear as dark areas in the image. Whenever a crack is present, it acts as an acoustic mirror and reflects the incident waves towards the receiver B. Thus the cracks should appear as bright bands against a dark field. The results presented in Fig. 2.23(a) for the CALB-1 specimen and in Fig. 2.24(a) for the CALB-2 specimen, show that this is indeed the case. The images were obtained by setting angle of incidence $\alpha=25^{\circ}$ and the azimuthal angles $\beta_{1}=\beta_{2}=135^{\circ}$ ). The polar backscattering images of cracks in the corresponding area for the CALB-1 and the CALB-2 specimens are shown in Figs. 2.23(b) and 2.24(b) respectively. In each case, a one-to-one correspondence is observed between the images obtained by the two methods. These results strongly suggest that the matrix cracks act as 'acoustic mirrors' in conjunction with the ply-interfaces and that the backscattered field is in fact a specular reflection of the ultrasonic beam caused by the presence of the cracks.

\subsubsection{Data Corroboration}

In the spirit of calibration, the matrix cracks detected by using the ply-by-ply ultrasonic NDE technique are compared with those detected by using optical microscopy and penetrant-enhanced X-radiography techniques described in the section 2.2.

Penetrant enhanced X-ray image of the 10-ply CALB-1 specimen is shown in Fig. 2.25(a). An enlarged view of a section of this specimen, shown by a dotted rectangle in Fig. 2.25(a), is shown in Fig. 2.25(b). The ultrasonic polar backscattering image and the schematic of the cracks detected optically at one of the edges, for the 


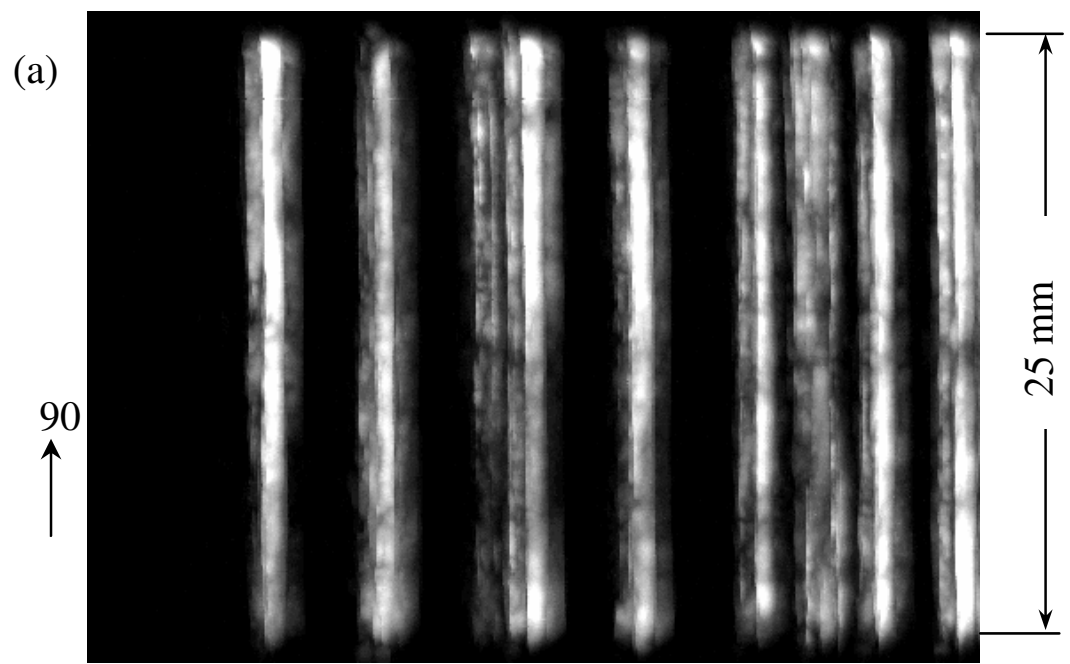

(b)

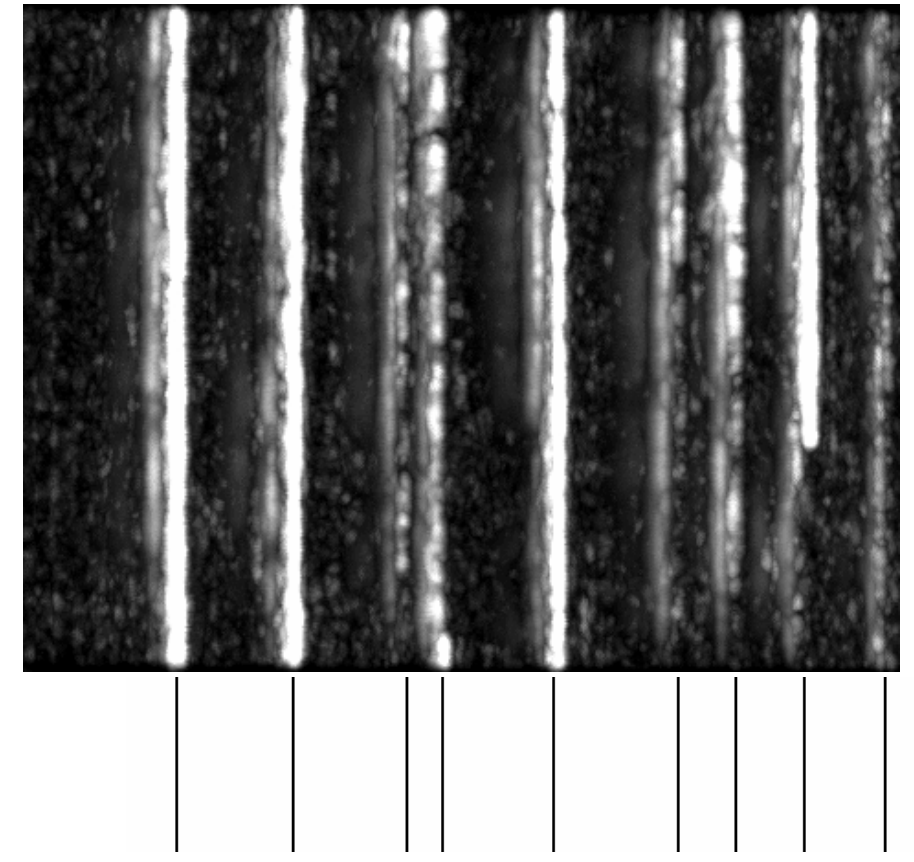

Fig. 2.23 Image of cracks in the $90_{8}$ ply-group of the 10-ply CALB-1 specimen. (a) As obtained by the arrangement shown in Fig. $2.21\left(\alpha=25^{\circ}, \beta_{1}=135^{\circ}, \beta_{2}=135^{\circ}\right)$, (b) polar backscattering $\left(\alpha=25^{\circ}\right)$, (c) schematic of the optically detected cracks at the edge of the specimen. 


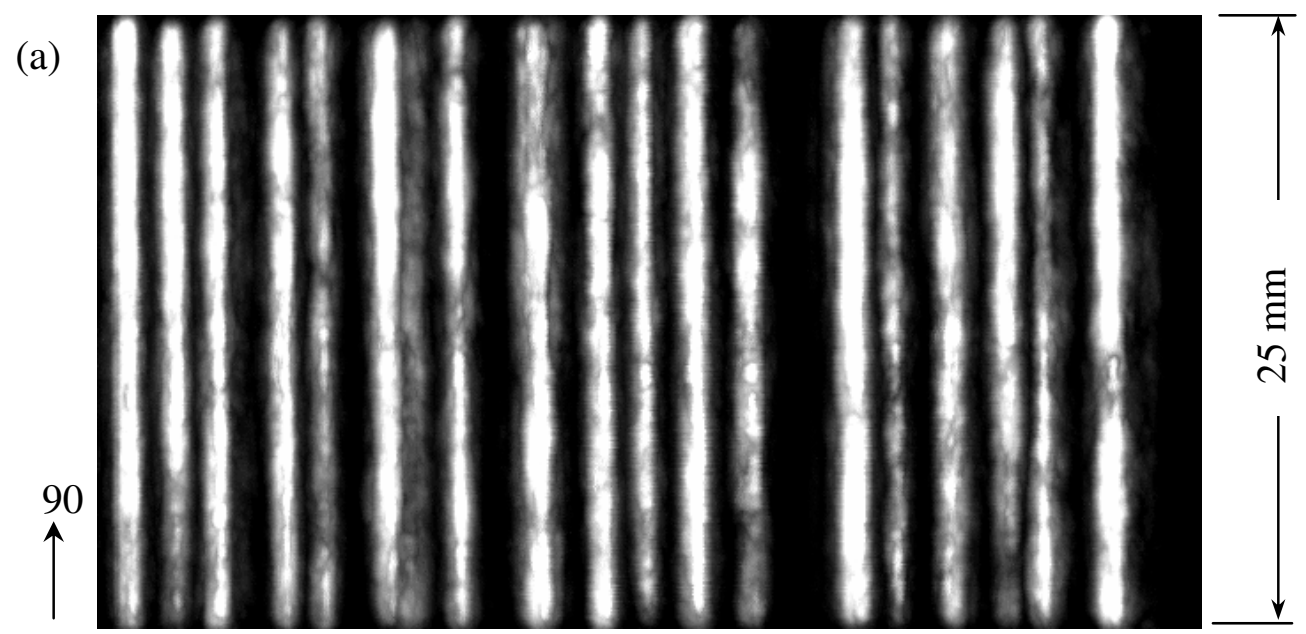

(b)

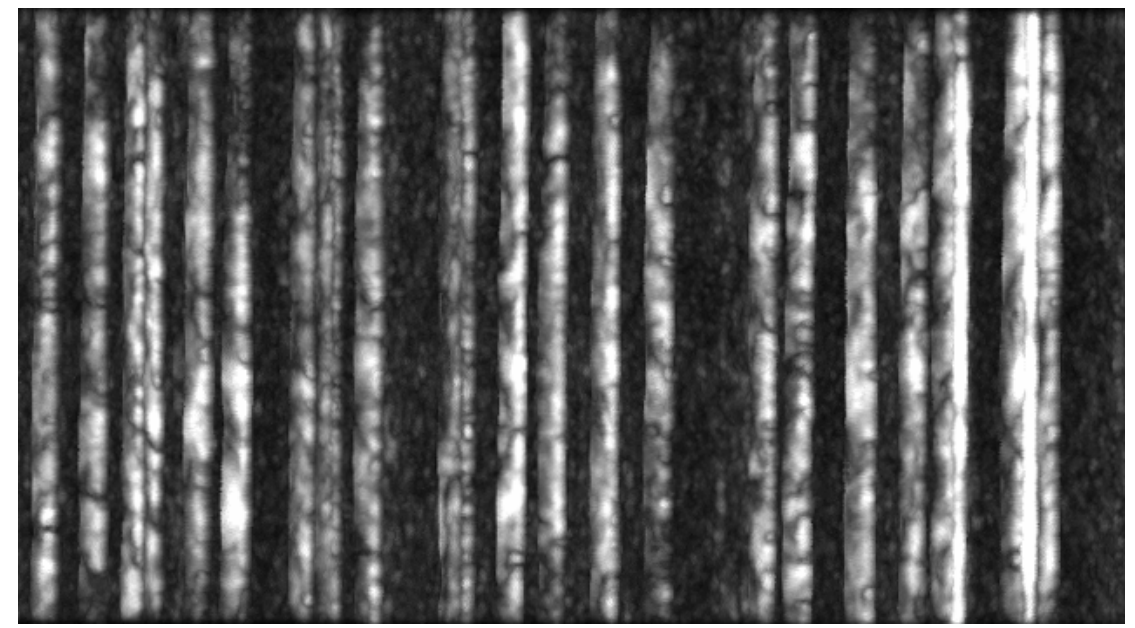

(c)

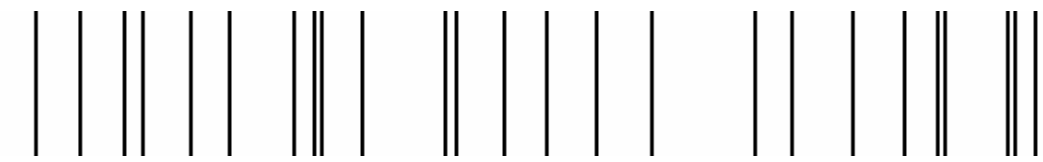

Fig. 2.24 Image of cracks in the $90_{6}$ ply-group of the eight-ply CALB-2 specimen. (a) As obtained by the arrangement shown in Fig. $2.21\left(\alpha=25^{\circ}, \beta_{1}=135^{\circ}, \beta_{2}=135^{\circ}\right)$, (b) polar backscattering $\left(\alpha=25^{\circ}\right)$, (c) schematic of the optically detected cracks at the edge of the specimen. 


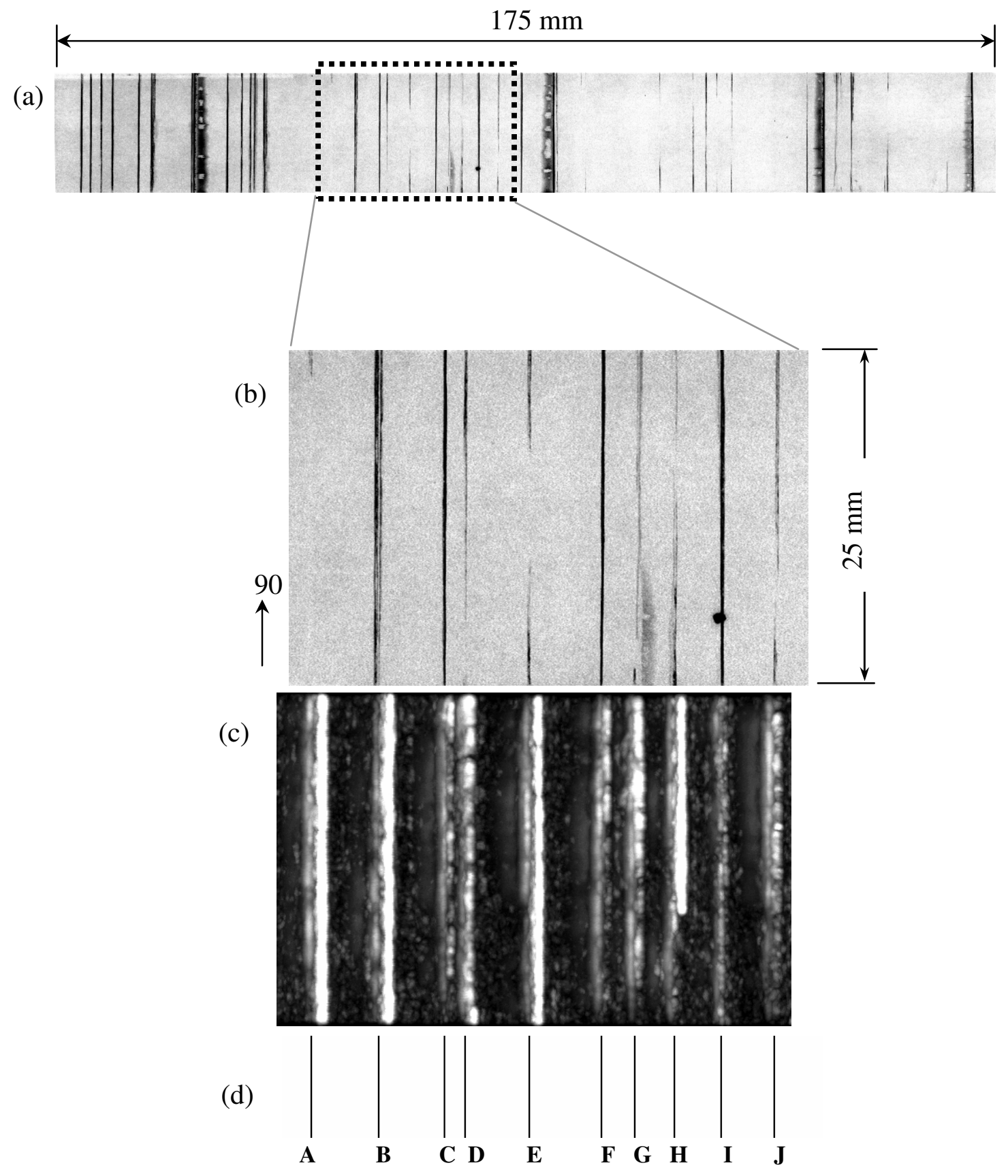

Fig. 2.25 Comparison between the images of the cracks in the $90_{8}$ ply-group of the CALB-1 laminate as obtained by different techniques. (a) and (b) penetrant enhanced X-ray images, (c) polar backscattering, (d) schematic of the optically detected cracks at the edge of the specimen. 


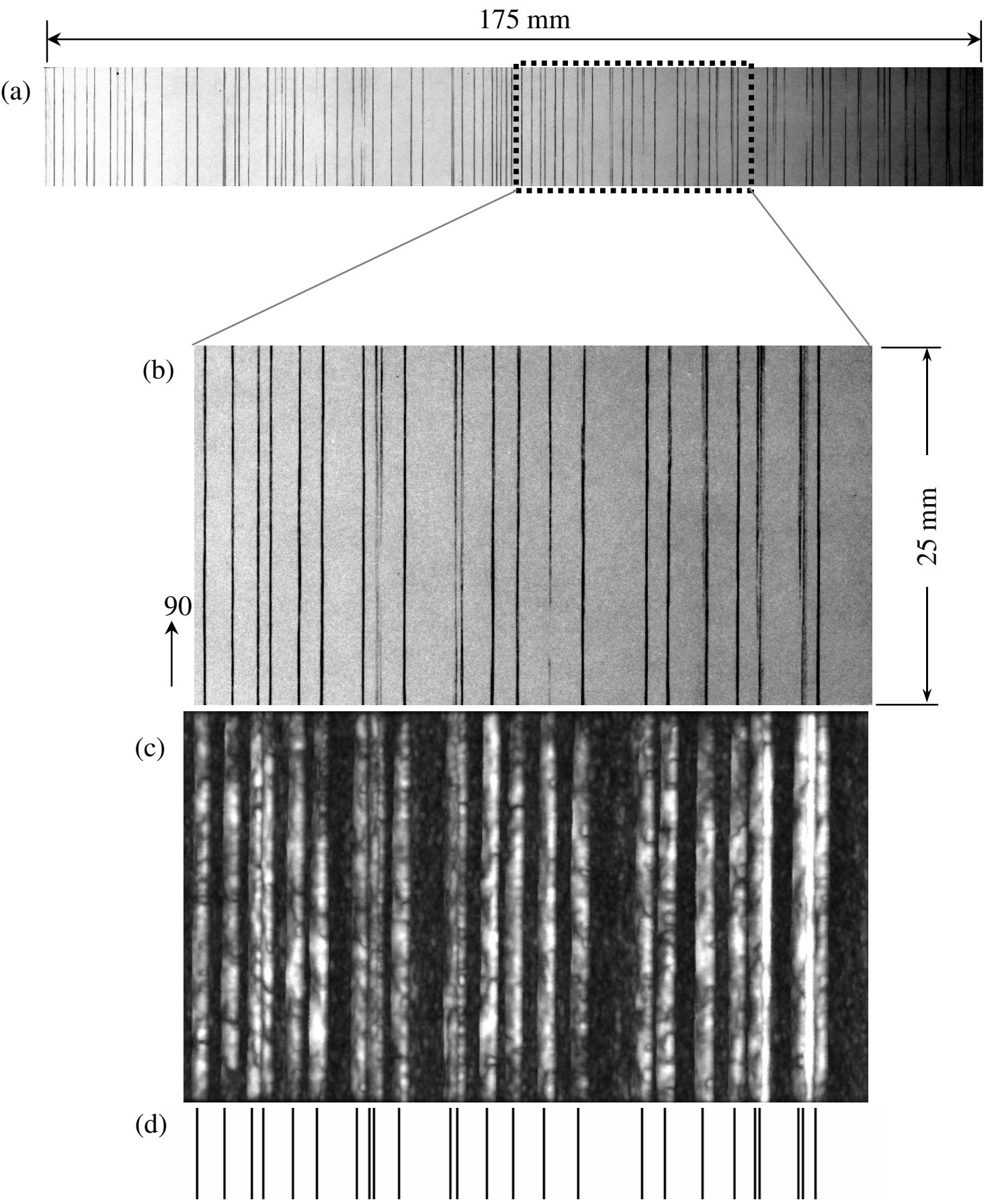

Fig. 2.26 Comparison between the images of the cracks in the $90_{6}$ ply-group of the

CALB-2 laminate as obtained by different techniques. (a) and (b) penetrant enhanced X-ray images, (c) polar backscattering, (d) schematic of the optically detected cracks at the edge of the specimen. 
same area, are shown in Figs. 2.25(c) and 2.25(d) respectively. A one to one correspondence is observed for the cracks detected by optical examination and ultrasonic technique. However, the X-ray image of the same area does not completely show all the cracks. Clearly, the X-ray opaque dye did not successfully penetrate the cracks marked A, D, E, H and J. X-ray image showing cracks in the 8-ply CALB-2 specimen is shown in Fig. 2.26(a). As before, an enlarged view of an area marked by the dotted rectangle in Fig. 2.26(a) is presented in Fig. 2.26(b). For this area, the ultrasonic image and the schematic of the optically detected cracks are shown in Figs. 2.26(c) and 2.26(d) respectively. In this case, the results using the three different techniques corroborate each other well.

A part of the X-ray image of the TAMU-1 (the highly damaged eight-ply [90/45/0/-45 $]_{\mathrm{s}}$ laminate), showing cracks in all the plies, is presented in Fig. 2.27. A subsection of this image, marked by dotted rectangle in Fig. 2.27, is magnified and presented in Fig. 2.28. At the bottom and on the left hand side of the Fig. 2.28, a schematic of the optically detected edge cracks is plotted. The cracks found in the $90^{\circ}$ plies are plotted as vertical lines, cracks found in the $45^{\circ}$ plies are plotted as lines at $45^{\circ}$, and so on. As may be seen, a fairly good correlation is observed between the cracks in the X-ray image (dark lines) and the optical image of the matrix cracks. However, the cracks in the $0^{\circ}, 90^{\circ}$ and the $45^{\circ}$ directions are not clearly visible in the X-ray image. The highly volatile X-ray opaque penetrant evaporates quickly from the outermost plies $\left(0^{\circ}\right)$, due to which, the cracks in these plies, running in the $0^{\circ}$ direction, are not clearly seen in the X-ray image. Optical examination revealed a large number of cracks were at the 


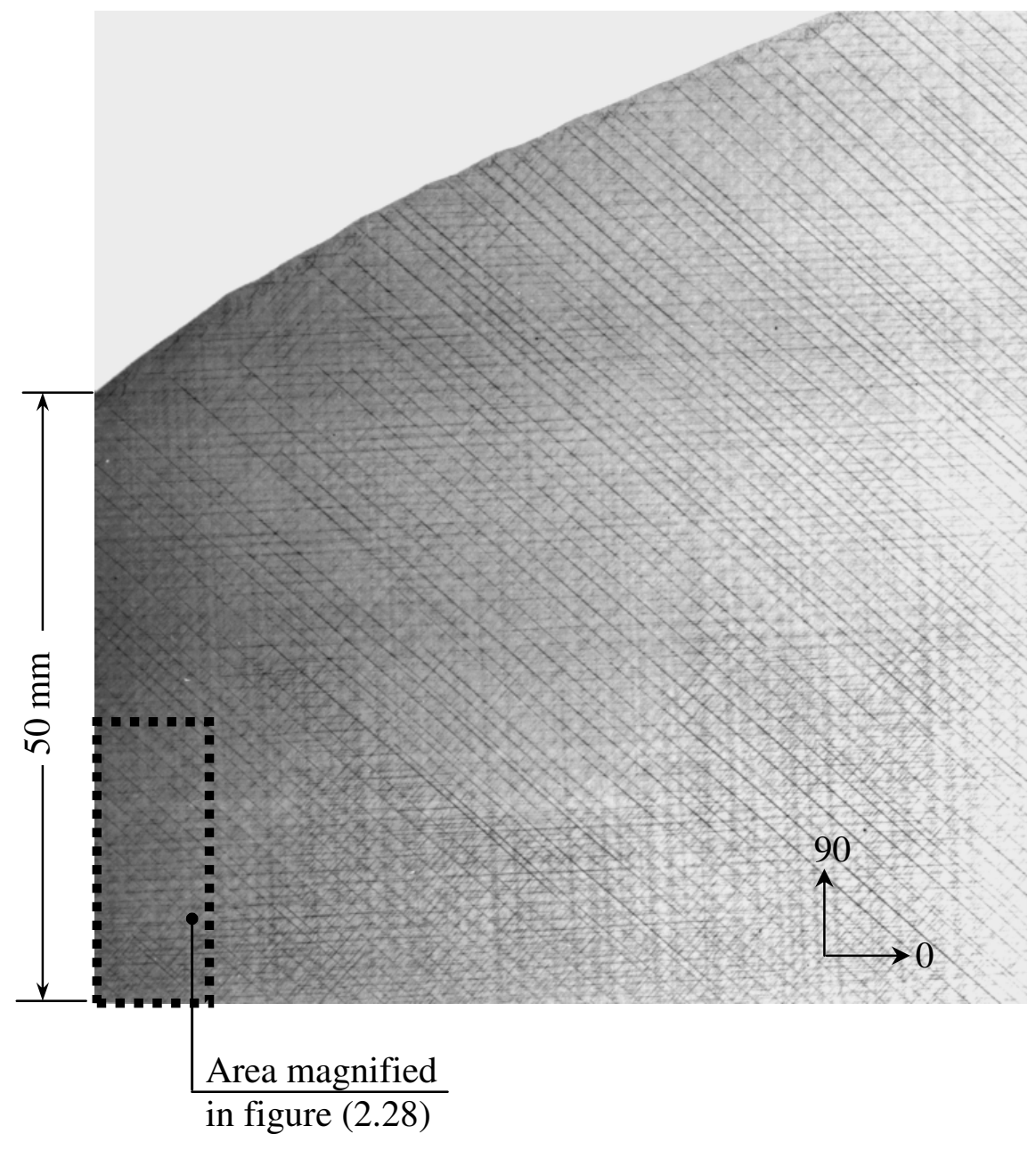

Fig. 2.27 An X-ray image of a part of the eight-ply TAMU-1 specimen showing matrix cracks (dark lines). 


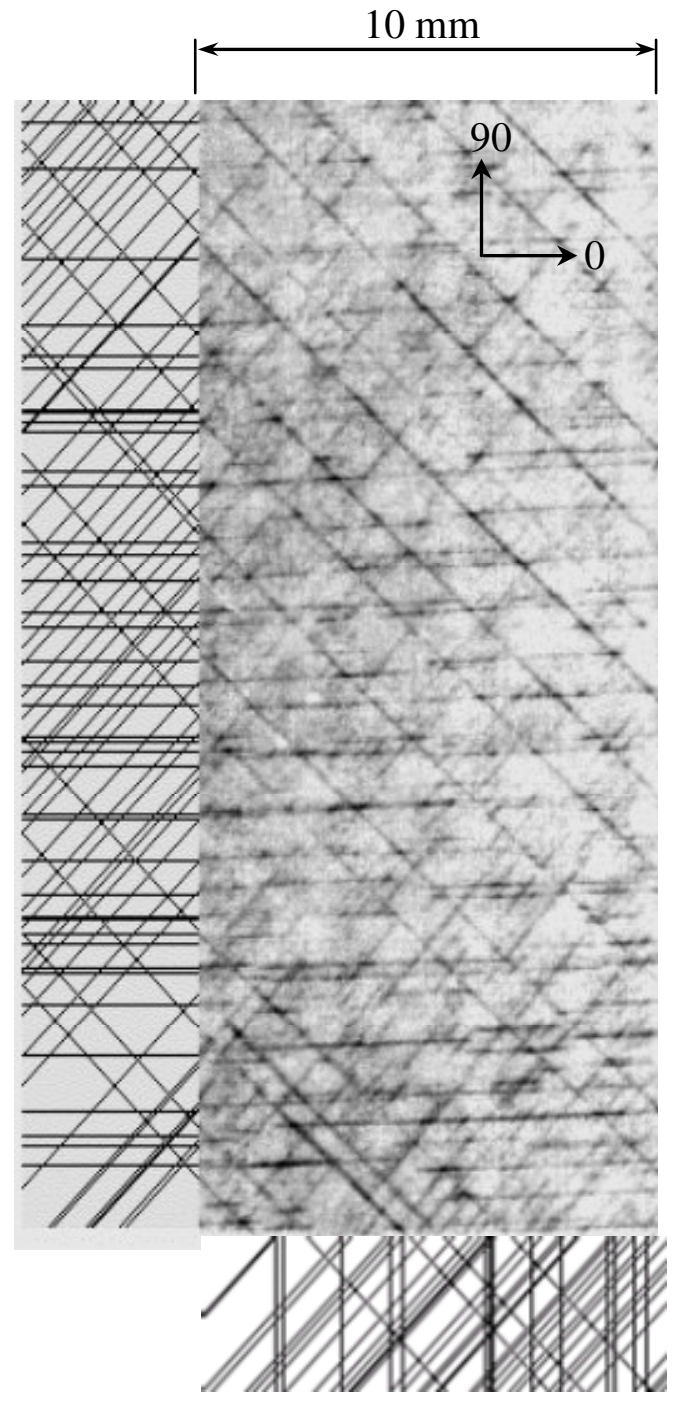

Fig. 2.28 A comparison of a part of the X-ray image of the TAMU-1 laminate with the schematic of the optically detected matrix cracks. 
edges of the second $\left(45^{\circ}\right)$, third $\left(90^{\circ}\right)$, sixth $\left(90^{\circ}\right)$ and the seventh $\left(45^{\circ}\right)$ plies. This was because of the presence of a high density of short cracks in these plies. A detailed discussion of short cracks is deferred to Chapter III. The short cracks are not clearly visible in the X-ray image shown in Fig. 2.28. This explains the lack of good correlation between the X-ray image and the schematic of the optically detected cracks in the $45^{\circ}$ and the $90^{\circ}$ directions.

A similar exercise was also conducted for all the other specimens. A comparison of the X-ray image and the schematic of the optically detected cracks for the TAMU-2 and the TAMU-3 specimens are presented in Figs. 2.29 and 2.30 respectively. For the clarity of communication, for each of these specimens, only small areas at one of the ends were chosen for comparison. A good correlation between the results obtained by the two methods is observed for the cracks running in the $90^{\circ}$ and the $-45^{\circ}$ direction. As in the case of TAMU-1 specimen, in these specimens too a large number of cracks in the $45^{\circ}$ and the $90^{\circ}$ plies were detected using optical microscopy. Again, the correlation between the X-ray image and the schematic of the optically detected edge cracks for the $0^{\circ}$ and the $45^{\circ}$ plies for both these specimens is not clear due to the presence of short cracks. Fig. 2.31 shows the comparison between the X-ray image and the schematic of the optically detected cracks in a small area of the TAMU-4 specimen. The area shown in Fig. 2.31 does not include either end of the specimen, due to which, the examination of $0^{\circ}$ plies was not possible. Hence, the cracks in the $0^{\circ}$ plies are not included in the schematic. Only two cracks running in the $45^{\circ}$ direction (see top left corner of the schematic) were observable at the edges. A good correlation is observed between the 


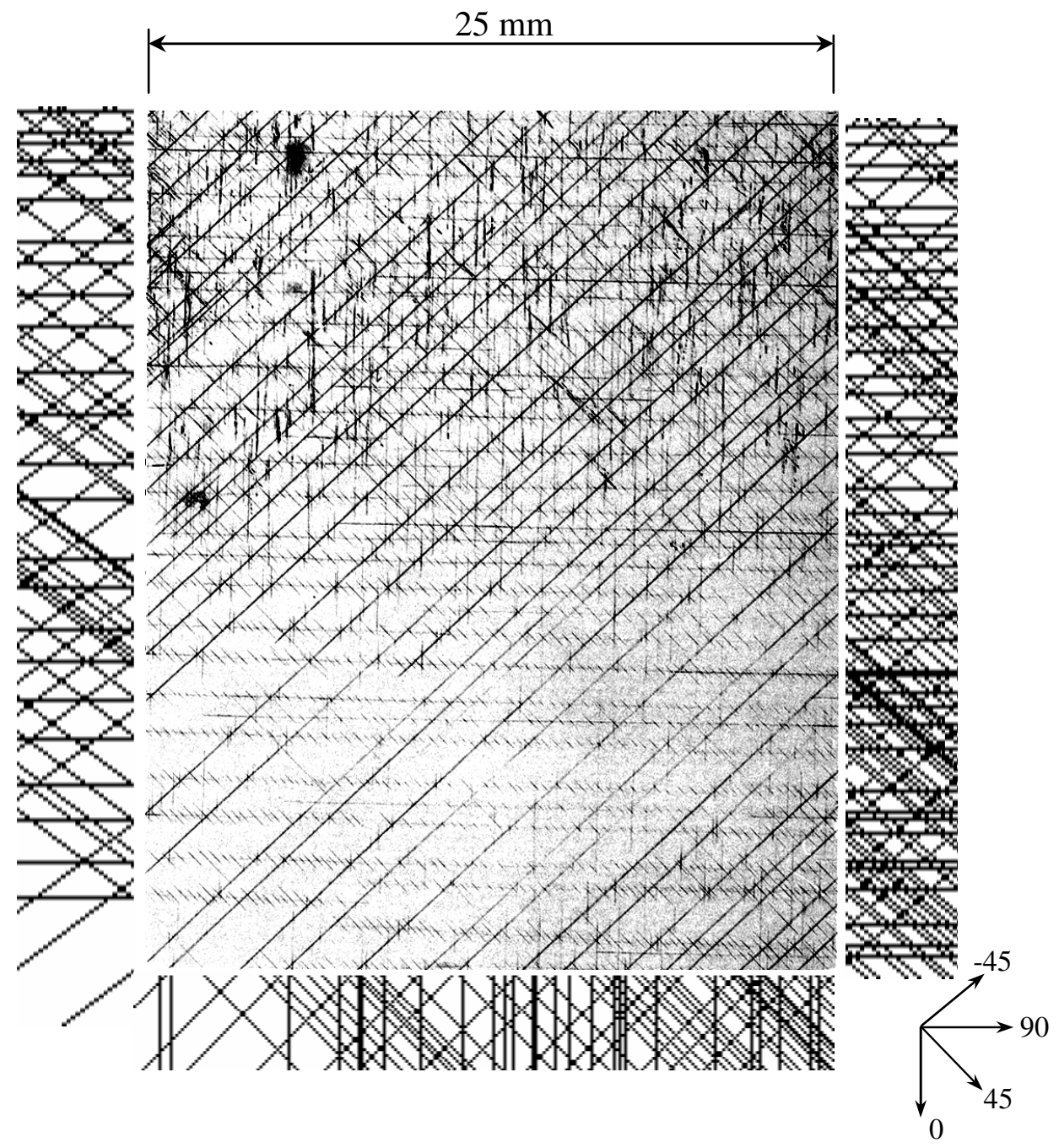

Fig. 2.29 A comparison of a part of the X-ray image of the TAMU-2 laminate with the schematic of the optically detected matrix cracks at the edges. 


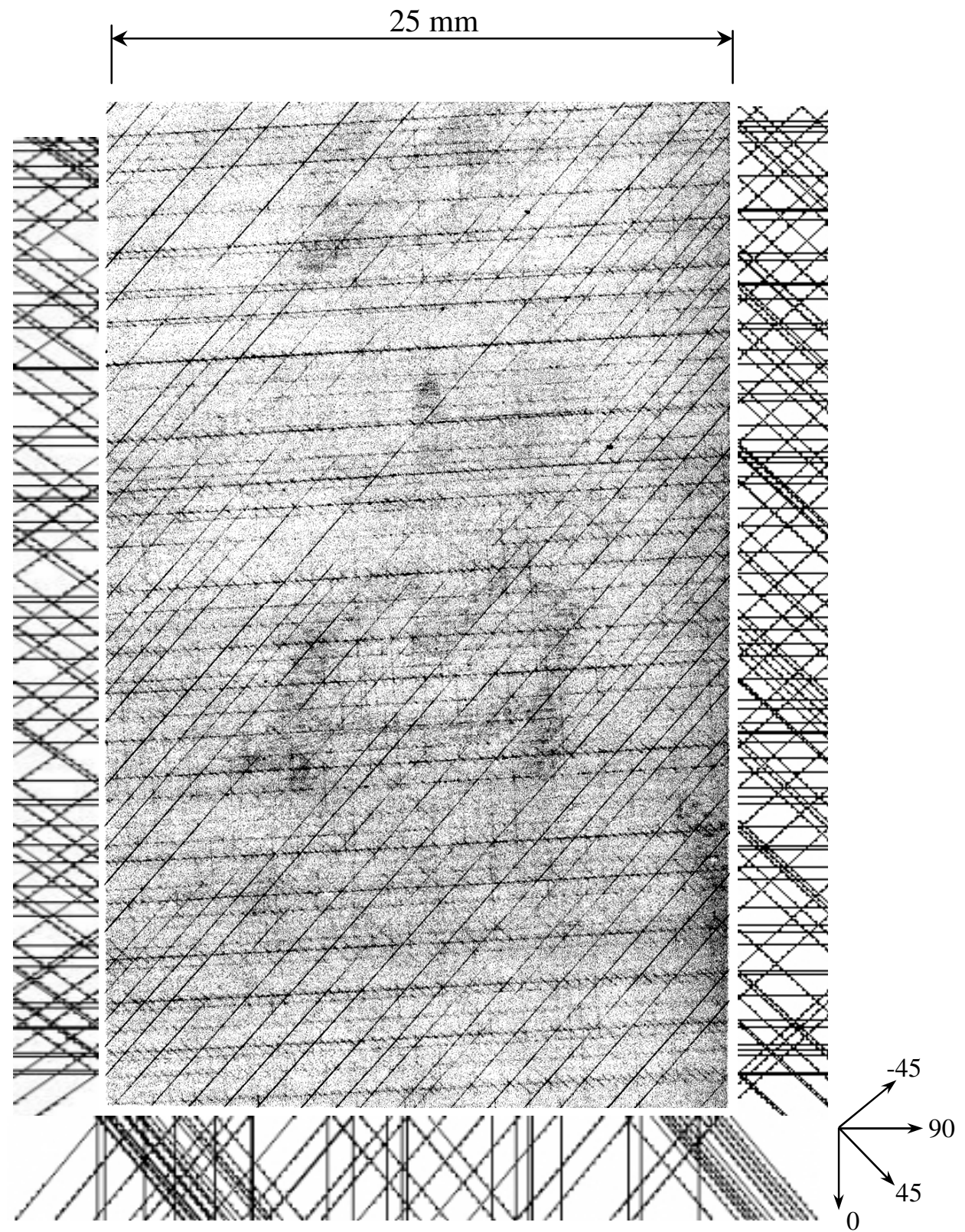

Fig. 2.30 A comparison of a part of the X-ray image of the TAMU-3 laminate with the schematic of the optically detected matrix cracks at the edges. 


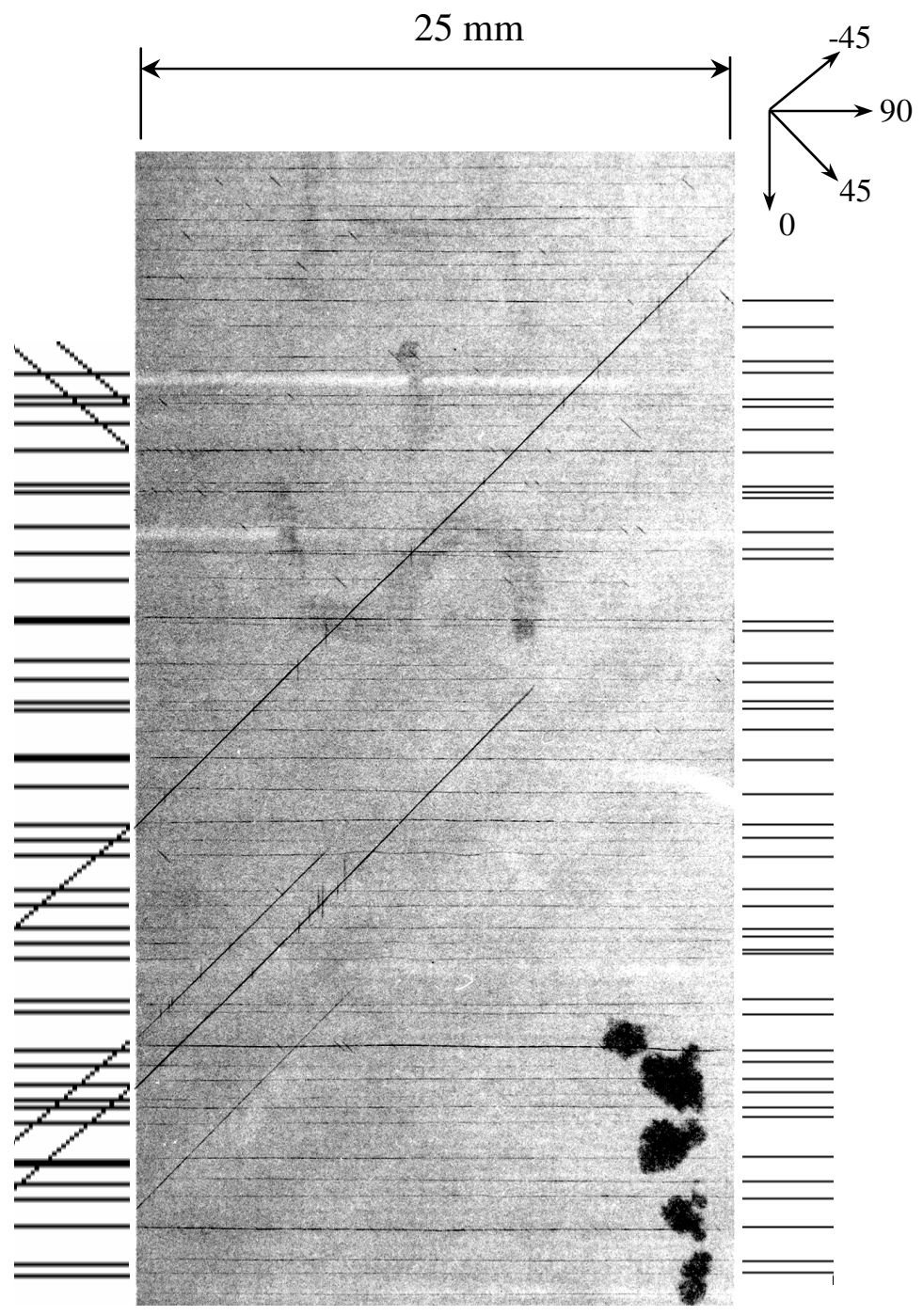

Fig. 2.31 A comparison of a part of the X-ray image of the TAMU-4 laminate with the schematic of the optically detected matrix cracks at the edges. 
X-ray image and the schematic of the optically detected cracks for the cracks in the $90^{\circ}$ and the $-45^{\circ}$ plies.

X-radiography results for the 18-ply TAMU-5 specimen did not show any cracks and hence only the optical data was available for comparison. A penetrant enhanced Xray image of the specimen is seen in Fig. 2.32. For this specimen, the comparison between the results obtained by using optical microscopy and ultrasonic polar backscattering technique are discussed in section 3.2 in Chapter III.

Next, for ease of comparison with the ultrasonic data, the X-ray image of the TAMU-1 specimen, shown in Fig. 2.27, was filtered to remove all lines except those corresponding to $-45_{2}$ cracks using the standard Kaiser filter with a Hamming window (Details of the filter and the filtering process is beyond the scope of this thesis. Readers may refer to Matlab documentation for the same); the results are presented in Fig. 2.33(a). The ultrasonic image of the cracks in the $-45_{2}$ ply-group obtained by polar backscattering method is shown in Fig. 2.33(b). When the image in Fig. 2.33(a) was superimposed on the image of Fig. 2.33(b) on a computer screen, an excellent (virtually a one-to-one) correspondence was observed. The comparison was better than can be seen in the printed version because some information is inevitably lost in going from the computer screen image to the printed version. A similar comparison was made between the filtered X-ray images and the ultrasonic images of cracks in the $-45_{2}$ ply-group of the TAMU-2, TAMU-3 and the TAMU-4 specimens. The X-ray and the ultrasonic images of the TAMU-2 specimen are presented in Figs. 2.34(a) and 2.34(b) respectively; for the TAMU-3 specimen the corresponding images are shown in Figs. 2.35(a) and 


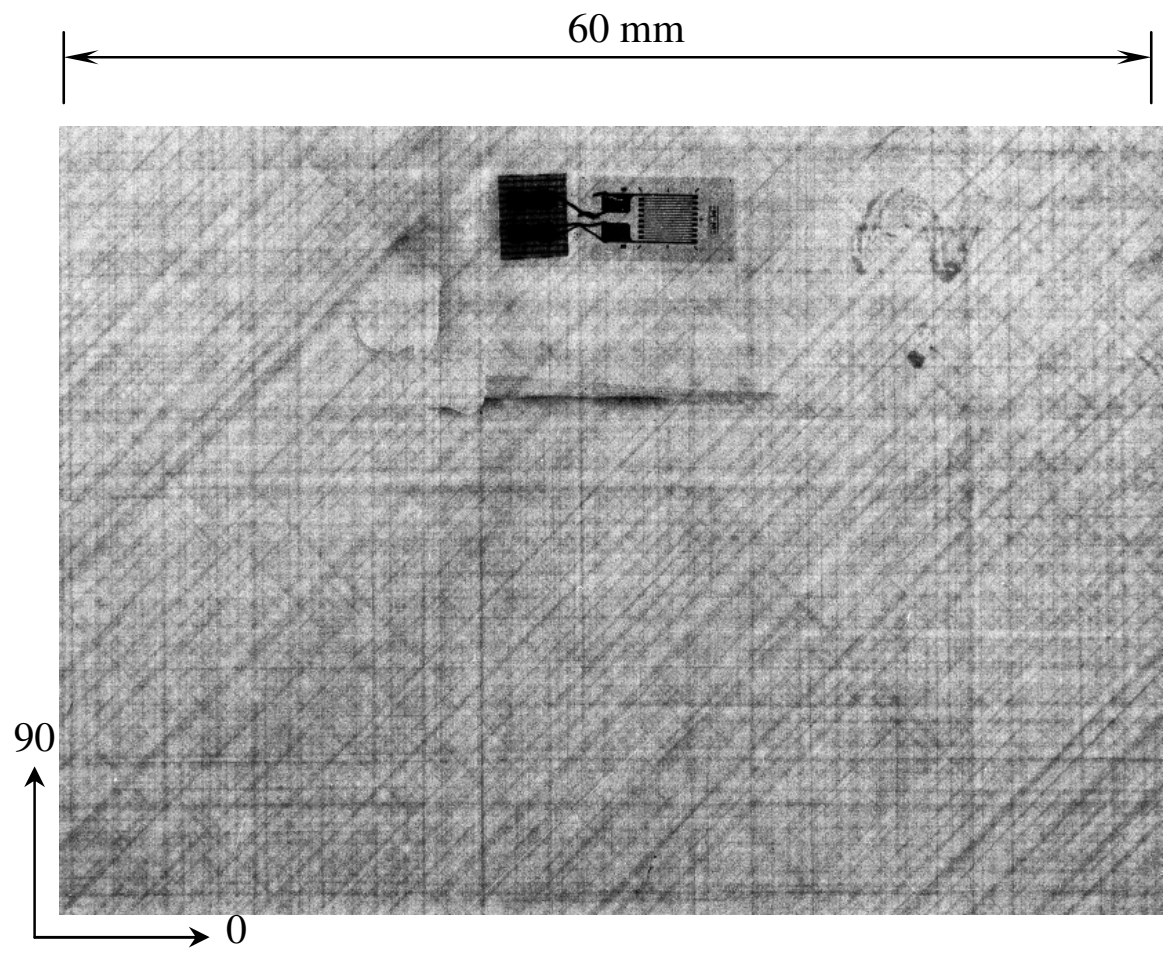

Fig. 2.32 X-ray image of a part of the TAMU-5 laminate. 


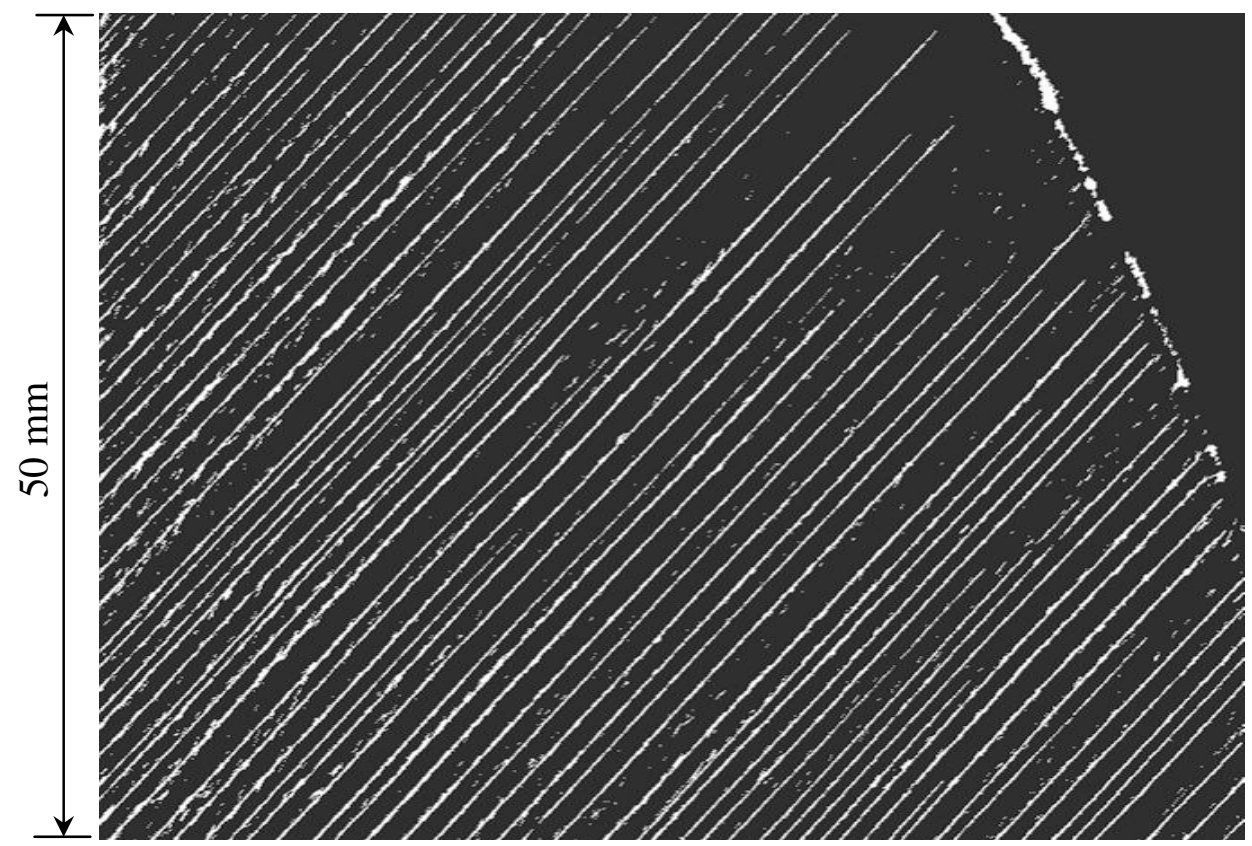

(a)

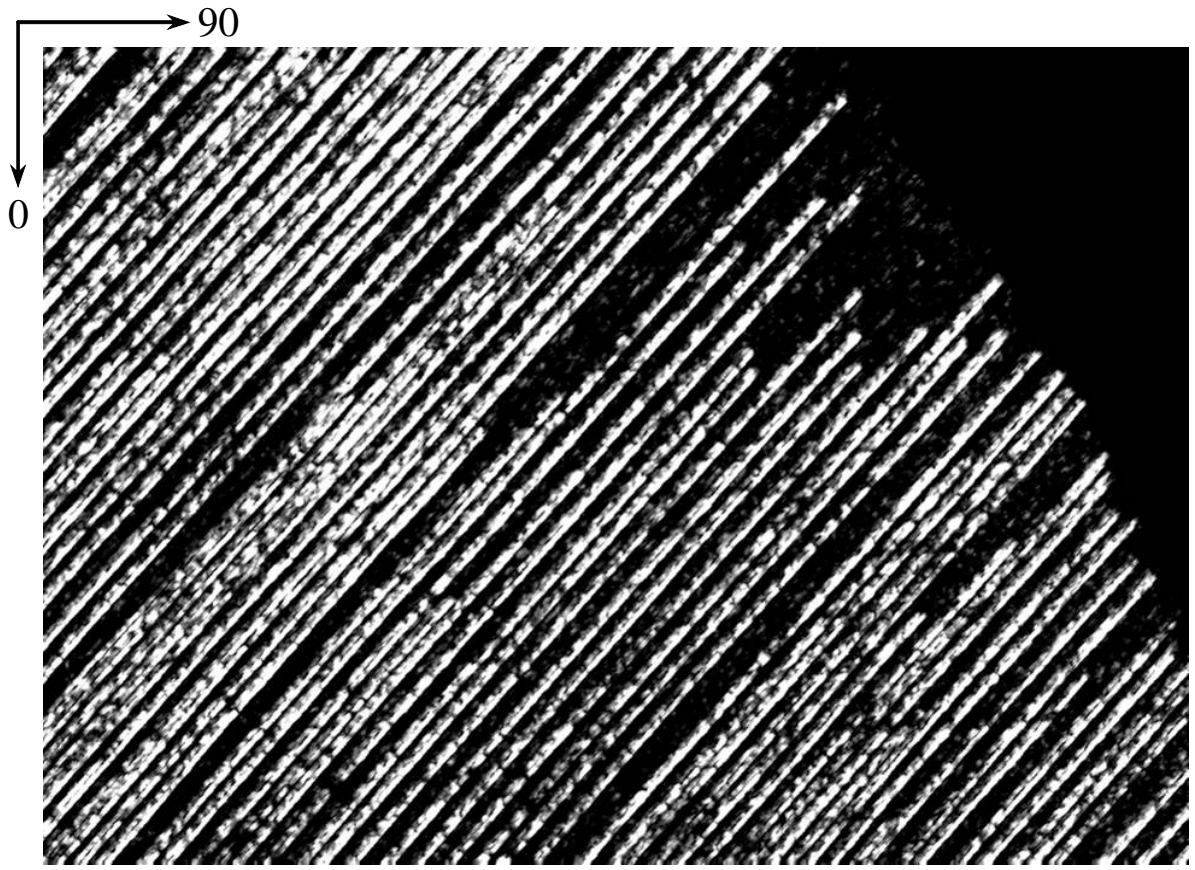

(b)

Fig. 2.33 (a) Filtered X-ray image, and (b) ultrasonic image, of cracks in the $-45_{2}$ ply-group of the TAMU-1 specimen. (The unfiltered X-ray image of the corresponding area can be seen in Fig. 2.27.) 


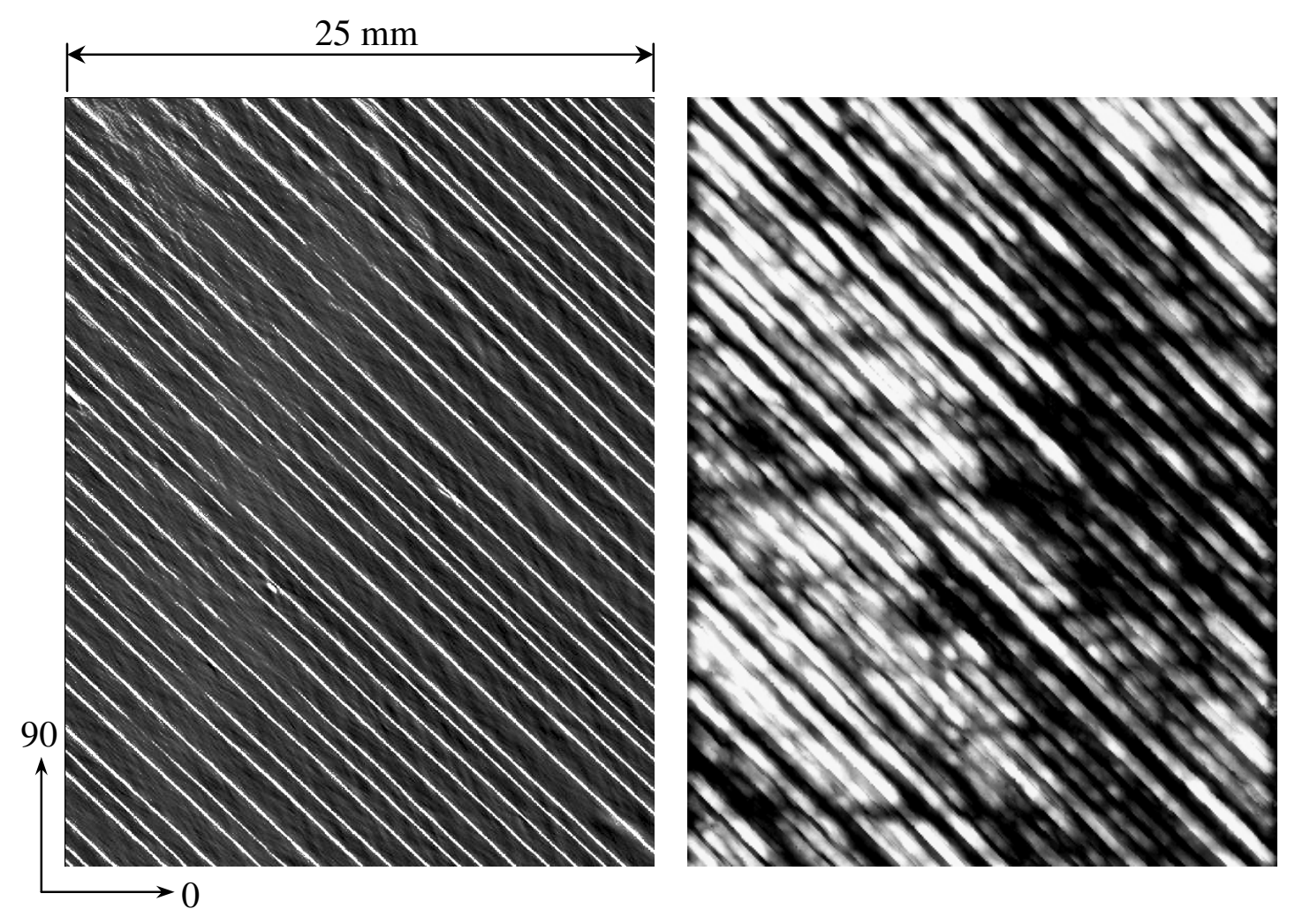

Fig. 2.34 (a) Filtered X-ray image, and (b) ultrasonic image, of cracks in the $-45_{2}$ ply-group of the TAMU-2 specimen. 

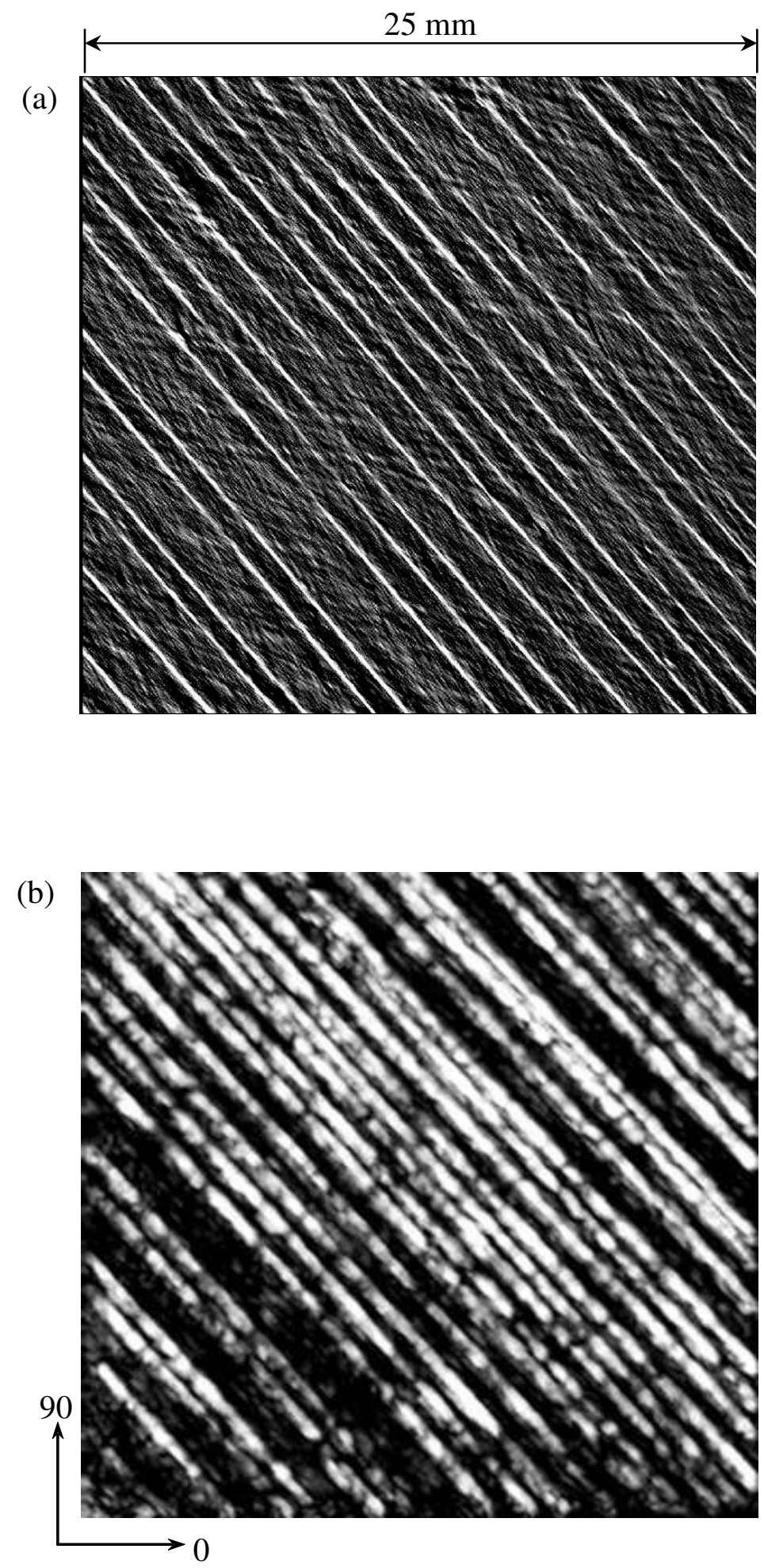

Fig. 2.35 (a) Filtered X-ray image, and (b) ultrasonic image, of cracks in the $-45_{2}$ ply-group of the TAMU-3 specimen. 


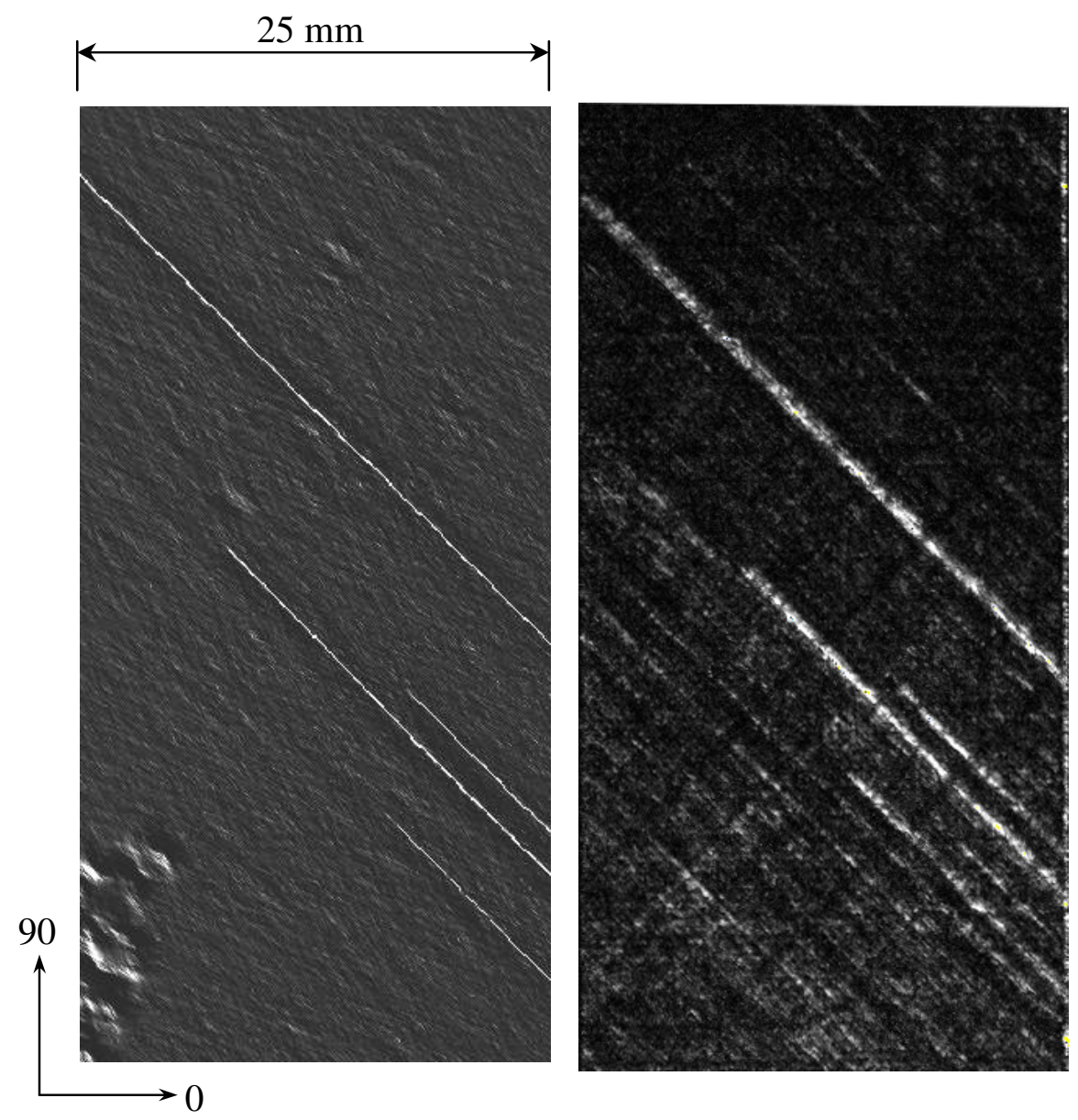

Fig. 2.36 (a) Filtered X-ray image, and (b) ultrasonic image, of cracks in the $-45_{2}$ ply-group of the TAMU-4 specimen. 
2.35(b) and in Figs. 2.36(a) and 2.36(b) for the TAMU-4 specimen. In all the cases an excellent correlation was observed filtered X-ray and the ultrasonic images. Comparison for TAMU-5 specimen is not shown for reasons discussed earlier.

In conclusion, the veracity of the results obtained with polar back-scattering technique has been established by comparison with the corresponding results obtained using X-radiography and optical microscopy techniques. 


\section{CHAPTER III}

\section{RESULTS AND DISCUSSION}

\subsection{TAMU-1 Specimen}

The images of cracks in the TAMU-1 specimen were obtained by scanning it with a $20 \mathrm{MHz}$ transducer oriented at $25^{\circ}$ angle of incidence. The results for this highly damaged specimen are presented in Fig. 3.1(a) through (h) for cracks in the first through eighth ply, respectively. Schematic maps of the optically detected matrix cracks in a given ply are placed below Fig. 3.1(a) and Fig. 3.1(h) and to the left hand side of Fig. 3.1(d) and Fig. 3.1(e). For these cases, the ultrasonic images show excellent (almost oneto-one) correlation with the optical data. A large number of cracks were detected optically at the edges for the second, third, sixth and the seventh plies each. However, for reasons that will become clear in the following the optical data was not plotted alongside the respective plies in Fig. 3.1.

Evidently, in order to detect the cracks in the second ply the acoustic beam has to twice travel through the cracks in the first ply; to detect the cracks in the third ply the acoustic beam has to twice travel through the cracks in the first and the second ply; and so on. Clearly, the task of detecting cracks in a given ply becomes progressively more difficult as its depth increases. Of course this difficulty level depends upon the cumulative damage in the plies leading up to the ply being interrogated.

In order to calibrate the polar backscattering technique against itself (selfcalibration), the cracks in the middle ply-group $\left(-45_{2}\right.$, fourth ply plus fifth ply) were 


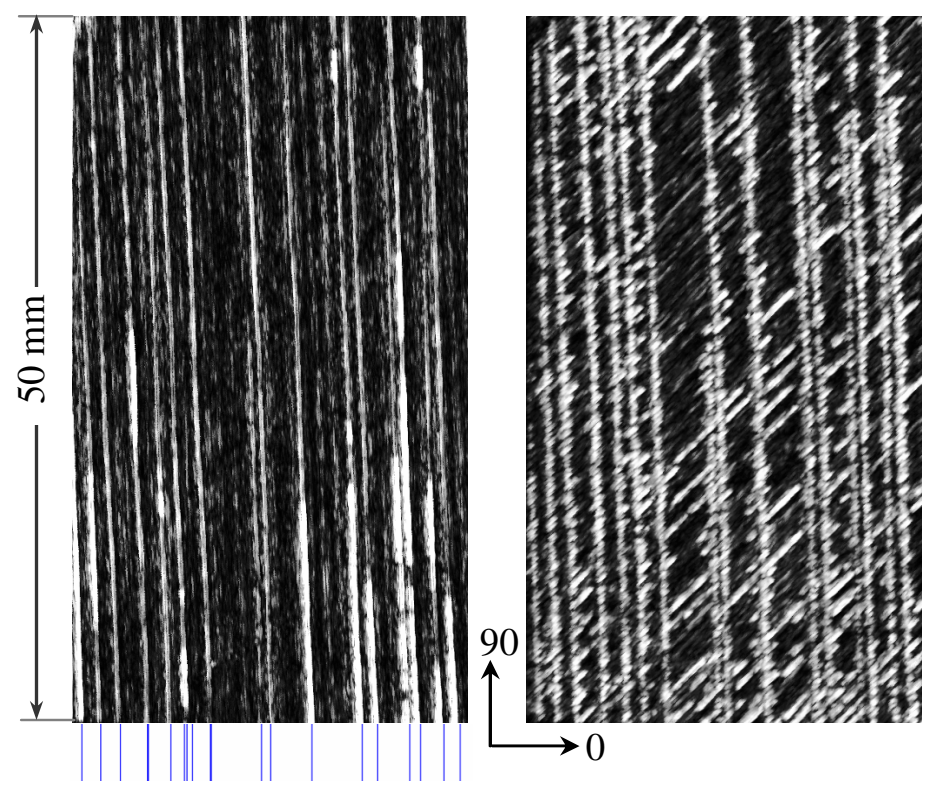

(a) First ply $\left(90^{\circ}\right)$

(b) Second ply $\left(45^{\circ}\right)$

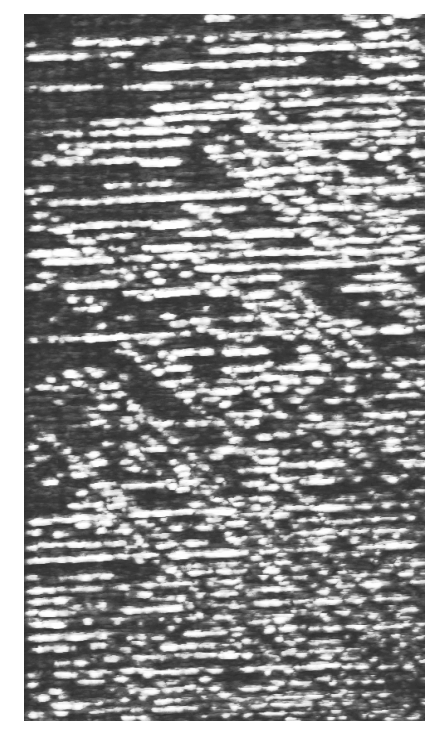

(c) Third ply $\left(0^{\circ}\right)$

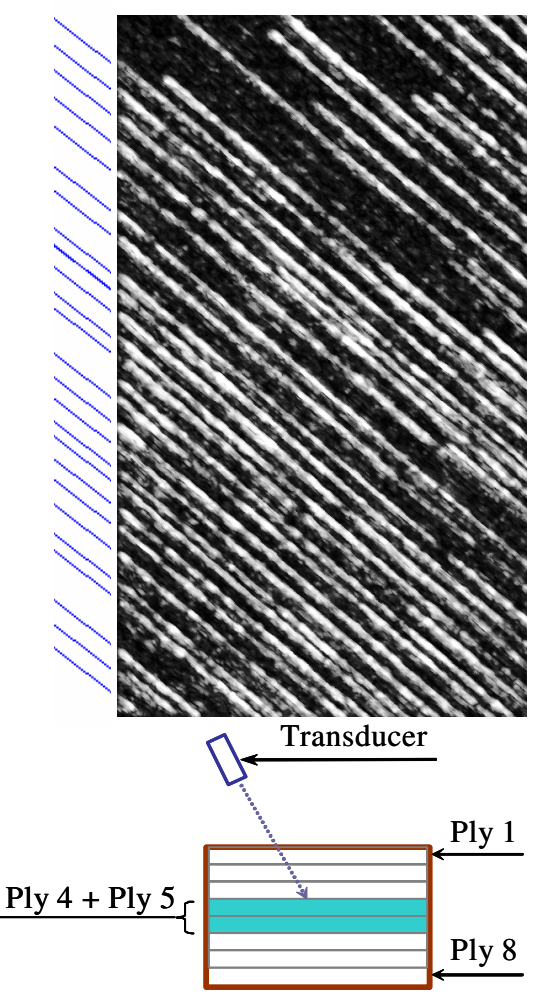

(d) Fourth + Fifth ply group $\left(-45^{\circ}\right)$
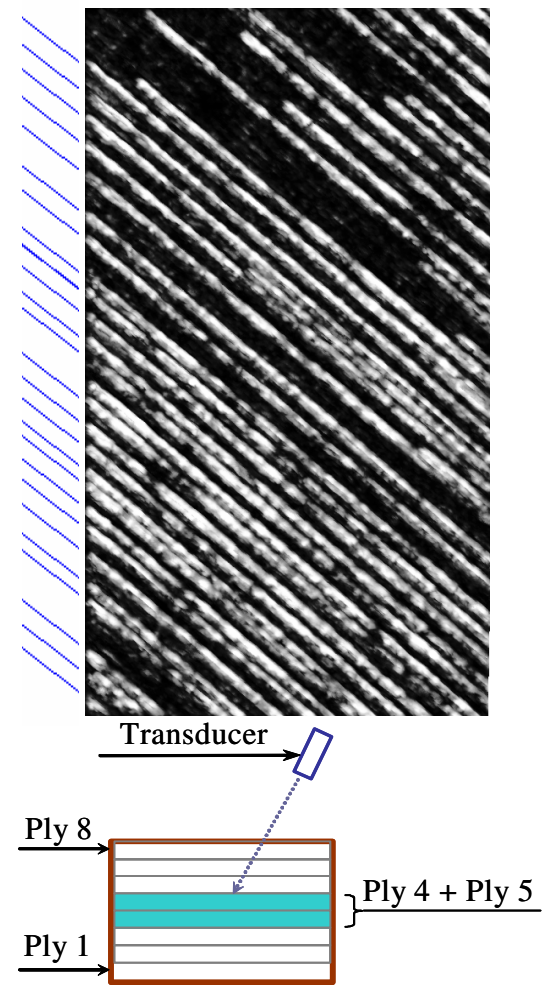

(e) Fourth + Fifth ply group from other side

Fig. 3.1 Ultrasonic images of cracks in the eight plies of the

$[90 / 45 / 0 /-45]_{\mathrm{s}}$ TAMU-1 specimen. (Angle of incidence $=25^{\circ}$ ) 


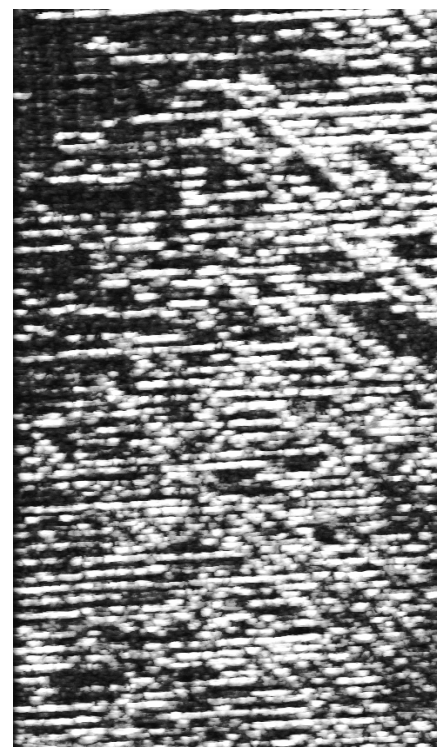

(f) Sixth ply $\left(0^{\circ}\right)$

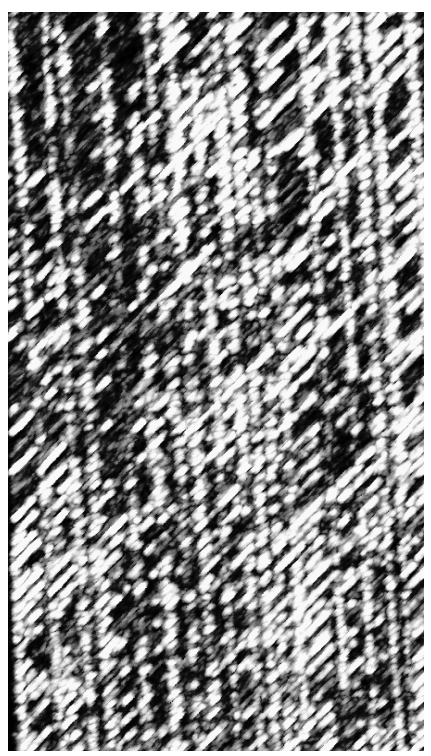

(g) Seventh ply $\left(45^{\circ}\right)$

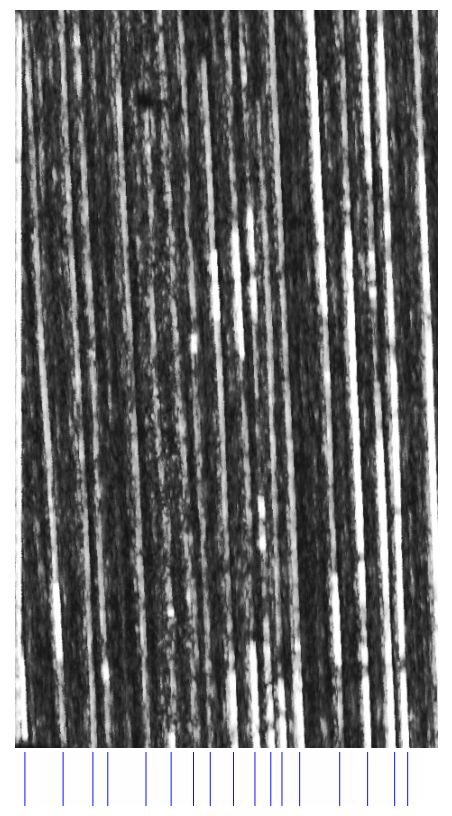

(h) Eighth ply $\left(90^{\circ}\right)$

Fig. 3.1 (continued) 
detected using two different paths: (1) In the first path, the interrogating acoustic beam travels through the first, second, and third plies on its way to the middle ply-group; and (2) In the second path, the beam travels through the eighth, seventh, and sixth plies on its way to the middle ply-group; see the schematic line drawings at the bottom of Figs. 3.1(d) and 3.1(e). The interrogating beam travels through two different damage states on its way to the ply being interrogated. The corresponding images of the matrix cracks are presented in Figs. 3.1(d) and 3.1(e), respectively. The agreement between the two images is excellent; there is a one-to-one correspondence observed between the two images, regardless of the damage in the intervening plies. This serves to self-calibrate the polar back scattering method for finding matrix cracks.

For this specimen, a typical time-domain signal in the absence of crack is shown in Fig. 3.2(a), while the time-domain signal in the presence of a crack is shown in Fig. 3.2(b). For both Figs 3.2(a) and 3.2(b) the transducer was oriented in such a way as to detect the cracks in the first ply $\left(90^{\circ}\right)$. The signal to noise ratio was found to be around $22 \mathrm{~dB}$, indicating a high degree of confidence in the detection of the cracks.

It is well known that, under practical loading conditions, the matrix cracks almost always run along the fiber direction. Accordingly, in the second ply we expect the cracks to run in the $45^{\circ}$ direction. To a casual observer the cracks in Fig. 3.1(b) might appear to be running in the $90^{\circ}$ direction. This is merely an illusion, and it may be explained as follows. A magnified image of the lower half of the second ply (as seen in Fig. 3.1(b)) is shown in Fig. 3.3(a). In the middle of the picture, one can clearly see cracks which originate at an interior point of the ply, run a short distance in the $45^{\circ}$ direction, and 


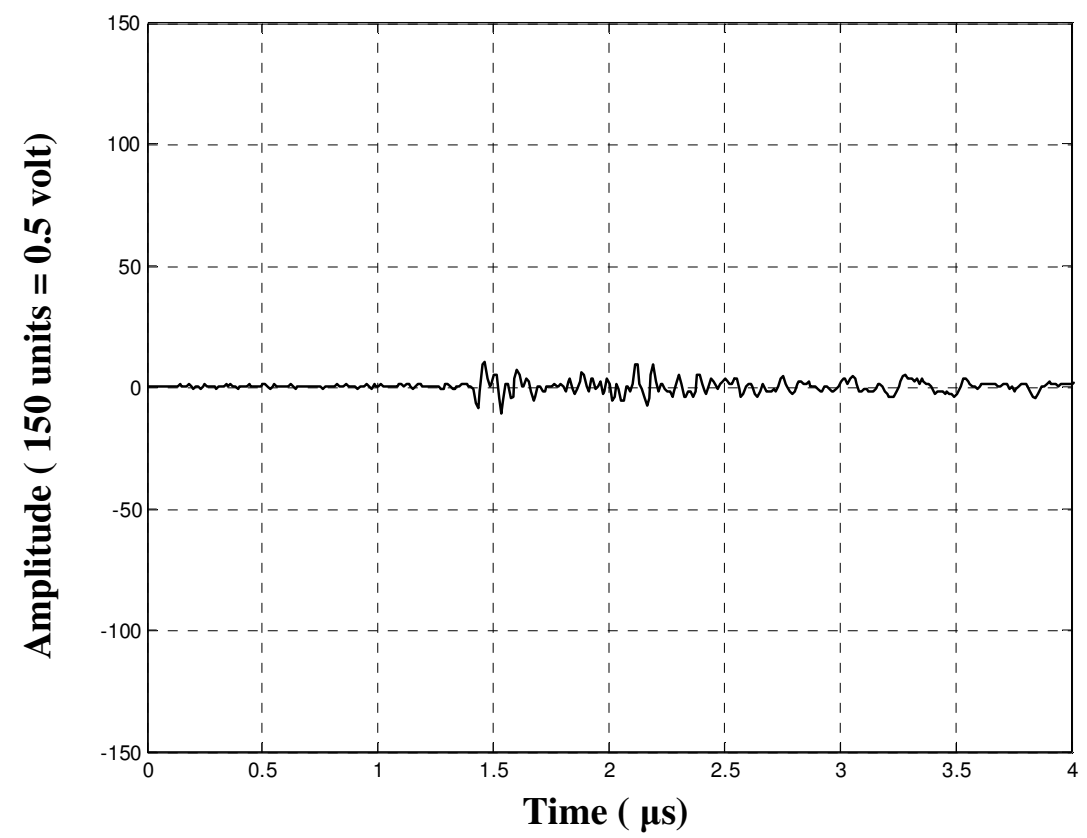

(a)

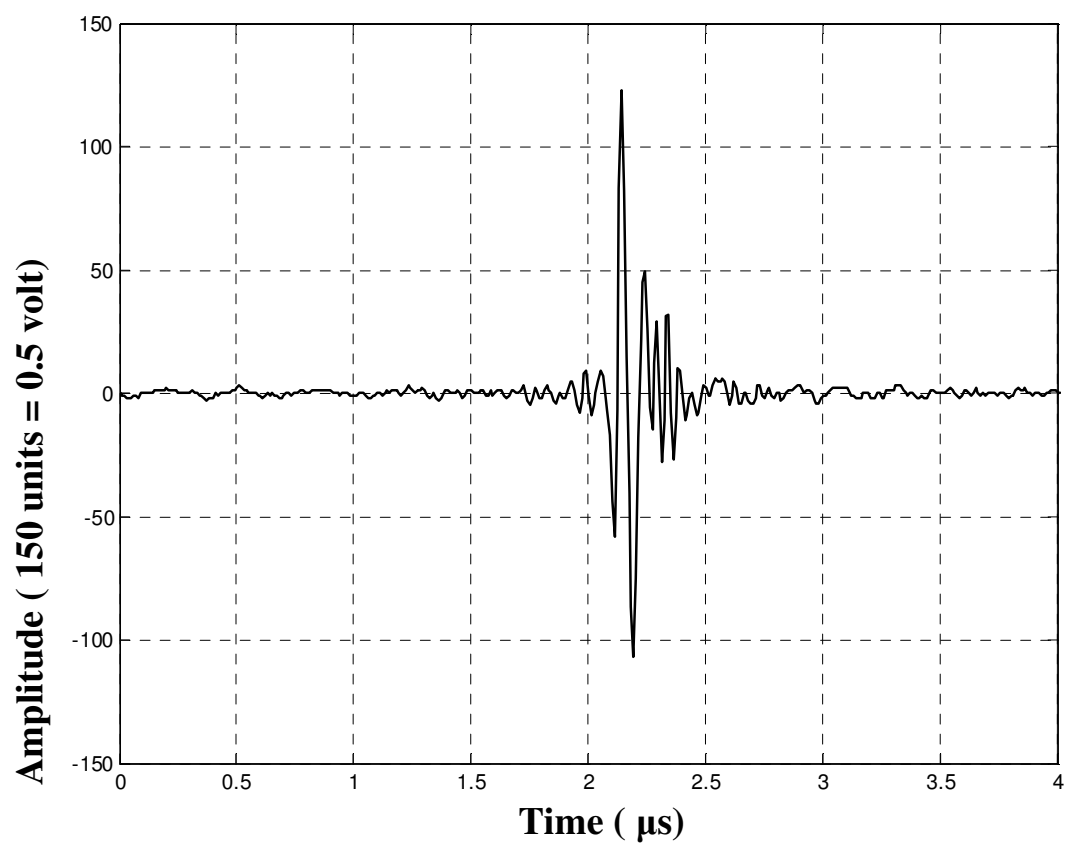

(b)

Fig. 3.2 Typical time domain signals; (a) in the absence of crack, (b) when a crack is insonified by the incident acoustic beam. (TAMU-1 specimen) 


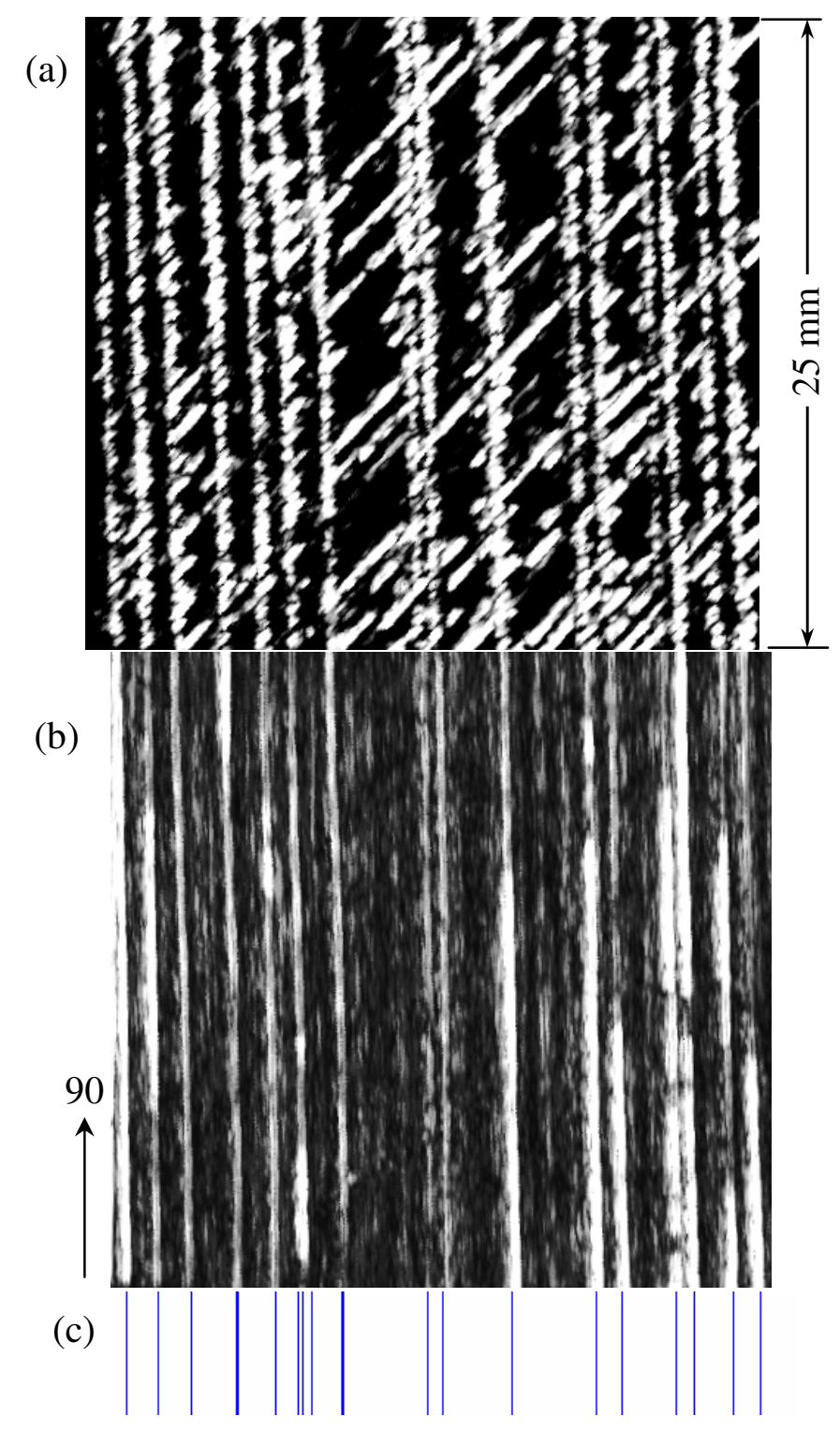

Fig. 3.3 (a) Short cracks in second ply $\left(45^{\circ}\right)$ of the TAMU-1 specimen, driven by cracks in the first ply $\left(90^{\circ}\right)$, (b) corresponding driving cracks in the first ply, (c) schematic of the optically detected cracks in the first ply. 
come to a stop. One can see the same phenomenon throughout Fig. 3.3(a). It is interesting to note that the cracks are not distributed in a random manner. Instead, they are stacked (or aligned) in the $90^{\circ}$ direction. Jamison et al. [31], Highsmith et al. [32], and more recently Lavoie and Adolfsson [33], have shown that these cracks owe their existence to the long (fully developed) cracks in the neighboring ply or ply-group (Lavoie and Adolfsson [33] call the neighboring plies as constraint plies and the short cracks in these plies as "stitch cracks" due to their characteristic appearance). In the present case, the short cracks in the second ply $\left(45^{\circ}\right)$ are produced by the long cracks in the first ply $\left(90^{\circ}\right)$.

To explain the formation of the cracks in the $45^{\circ}$ ply, consider a simple case of a [90/45] laminate loaded in a direction transverse to the $90^{\circ}$ ply. In the uncracked condition, both the plies have a uniform distribution of stress along the length of the laminate. Whenever a matrix crack appears in the $90^{\circ}$ ply, the ply can no longer carry any load at the location where the crack is present. There is a transfer of this load to the $45^{\circ}$ ply and equilibrium is maintained. It is well known that the load transfer is localized to a small distance around the crack. Beyond this distance the stress distribution is the same as it was before cracking. The additional load on the $45^{\circ}$ ply increases the stress in this ply and when the component of the increased stress in the principal direction exceeds the matrix cracking stress, cracks start to appear in the $45^{\circ}$ ply. However, since the increased stresses are highly localized, the condition for crack formation is restricted to only a short distance from the crack in the $90^{\circ}$ ply, thus leading to short cracks in the $45^{\circ}$ ply. Another plausible explanation for the formation of short cracks is the high stress 
concentration at the crack-tip of a fully developed crack in the $90^{\circ}$ ply. Again, since the crack-tip stress concentration is also highly localized, it will increase the stress in the $45^{\circ}$ ply only locally near the area of the crack tip and give rise to short cracks. This line of thought appears more convincing in the case of laminates with many plies, wherein the load transfer from a cracked ply to the remaining uncracked plies in itself may not be significant enough to cause the formation of short cracks.

With either of the above explanations, it follows that there should be a strong correlation between the spatial location of the $90^{\circ}$ long cracks in the first ply and the spatial location of the $45^{\circ}$ short cracks in the second ply. It is satisfying to note that such a correlation indeed exists, as may be seen when the lower (corresponding) half of Fig. 3.1(a), reproduced in Fig. 3.3(b), is compared with Fig. 3.3(a). A schematic of the optically detected cracks in the first ply is shown in Fig. 3.3(c). The correlation is even more conspicuous if one observes the partial cracks in the first ply and the corresponding short cracks in the second ply. This is illustrated in Fig. 3.4. For the ease of presentation, the upper halves of Figs. 3.1(a) and 3.1(b) showing cracks in the first and the second plies are reproduced in Figs. 3.4(a) and 3.4(b) respectively. Partial cracks in the first ply are contained in areas marked by rectangles A and B (Fig. 3.4(a)). Corresponding areas in the second ply containing short cracks are marked by rectangles $\mathrm{C}$ and D respectively (Fig. 3.4(b)). Enlarged views of the cracks in the area A (first ply) and the corresponding short cracks in the area $\mathrm{C}$ (second ply) are presented in Fig. 3.4(c). Similarly, cracks in corresponding areas B (first ply) and D (second ply) are shown in 3.4(d). In Fig. 3.4(c), the cracks in the first ply marked A1 and A4 are partial cracks 


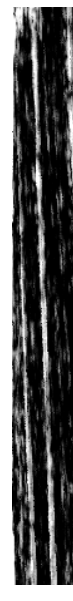

(a)
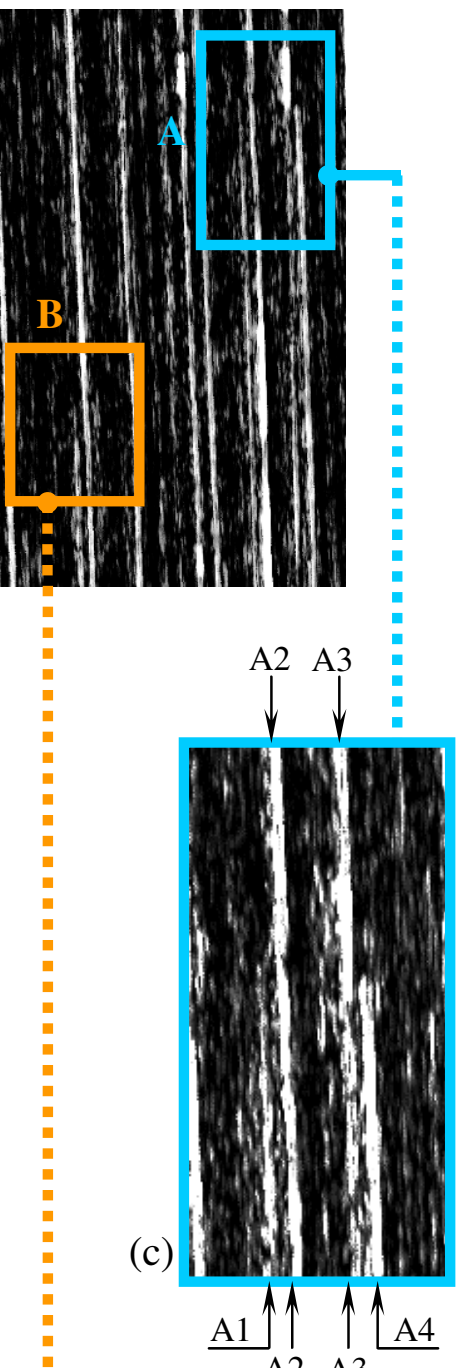

A2 A3

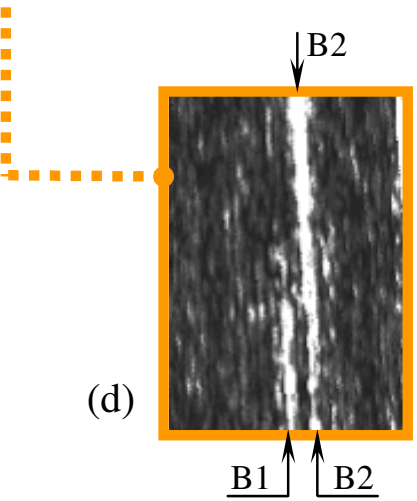

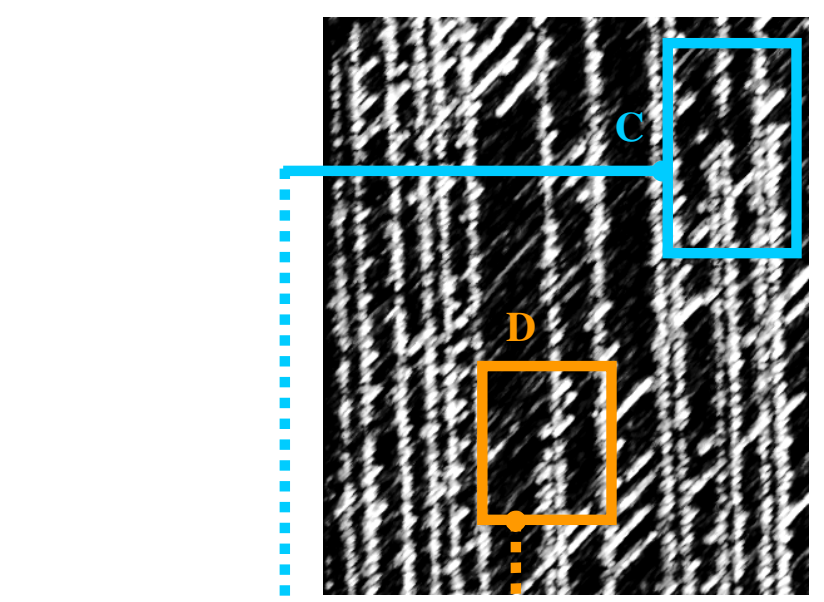

(b)

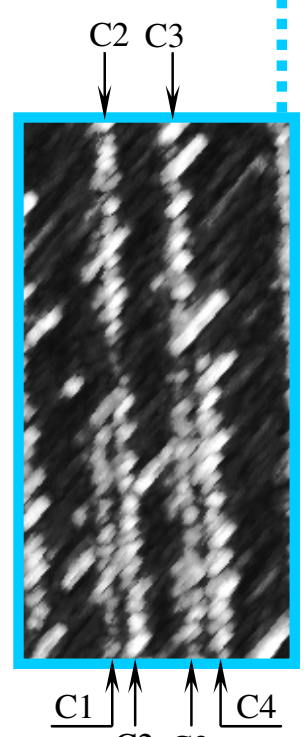

$$
\text { ㅁ. }
$$

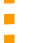

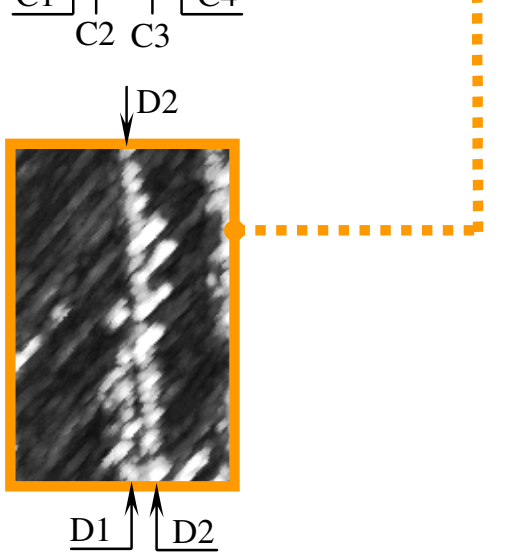

Fig. 3.4 (a) Cracks in first ply $\left(90^{\circ}\right)$, (b) short cracks in the corresponding area in the second ply $\left(45^{\circ}\right)$, (c) enlarged views of the areas marked $A$ and $C$, (d) enlarged views of the areas marked $B$ and $D$. 
terminating around the central area of the rectangle, whereas, cracks marked A2 and A3 extend throughout the length of the rectangle. In the second ply, corresponding to the cracks A1, A2, A3 and A4, four columns of short cracks are marked as C1, C2, C3 and $\mathrm{C} 4$ respectively. It is seen that, the short cracks in the columns C2 and C3 (caused due to the cracks $\mathrm{A} 2$ and $\mathrm{A} 3$ respectively) extend over the entire length of the rectangle while the short cracks in columns $\mathrm{C} 1$ and $\mathrm{C} 4$ (caused due to the cracks $\mathrm{A} 1$ and $\mathrm{A} 4$ respectively) exist only as long as the partial cracks A2 and A3 exist in the first ply. A similar phenomenon is observed in Fig. 3.4(d), where the cracks marked B1 and B2 in the first ply give rise to columns of short cracks marked D1 and D2 respectively in the second ply. This experimental data provides overwhelming evidence in support of the fact that the short cracks are caused by the fully developed cracks in the adjacent plies.

Attention is now turned to the short cracks in the $0^{\circ}$ direction in the third and the sixth plies which brace the $-45_{2}$ ply-group. A similar detailed examination of the data was carried out. Once again it was found that there is a strong correlation between the spatial location of the long cracks in the $-45_{2}$ ply-group and the short cracks in the third and the sixth plies. The same comments apply to the short cracks in the seventh ply driven by the long cracks in the eighth ply. Because of the presence of a high density of short cracks in the second, third, sixth and the seventh plies, there were obvious difficulties in trying to compare the ultrasonic results with those obtained by using optical microscopy. This explains the lack of observable correlation between the cracks in the $45^{\circ}$ and the $90^{\circ}$ directions as seen in the X-ray image in and the corresponding schematic of the optically detected edge cracks in Fig. 2.28 and the absence of 
schematics of the optically detected cracks at the edges of the second, third, sixth and the seventh plies in Fig. 3.1.

\subsection{TAMU-2, TAMU-3 and TAMU-4 Specimens}

On one side, the TAMU-2 was covered with an aluminum liner and the TAMU-3 specimen with a Tedlar liner while the other side of both the specimens showed a rough surface. The details of the liners have been discussed earlier in section 2.1. The TAMU4 specimen did not have liner on either surfaces, but both ply-surfaces showed surface roughness.

When the specimens were scanned for cracks from the rough side, proper gating of the time-domain signal was required to separate the signals reflected by the cracks from the backscattered signal due to surface roughness. As an example a time-domain signal for the TAMU-4 specimen is presented in Fig. 3.5. The transducer was oriented to detect cracks in the first ply through the rough surface. The backscattered signal due to the surface roughness was found to be present for an initial period of about $0.4 \mu \mathrm{s}$. If a gate for collecting the data is improperly placed in such a way that it includes the signal due to the rough surface, the resulting image will show a large amount of noise making it difficult to discern cracks from the artifacts due to surface roughness. To demonstrate this, three gates of different length were set on the time domain signal as shown in Fig. 3.6(a). Gate A spans the entire time-domain signal and the corresponding image obtained is shown in Fig. 3.6(b). The length of the Gate B was set to $400 \mathrm{~ns}$ and was placed in such a way that it spans the entire signal due to the surface roughness gathers. 


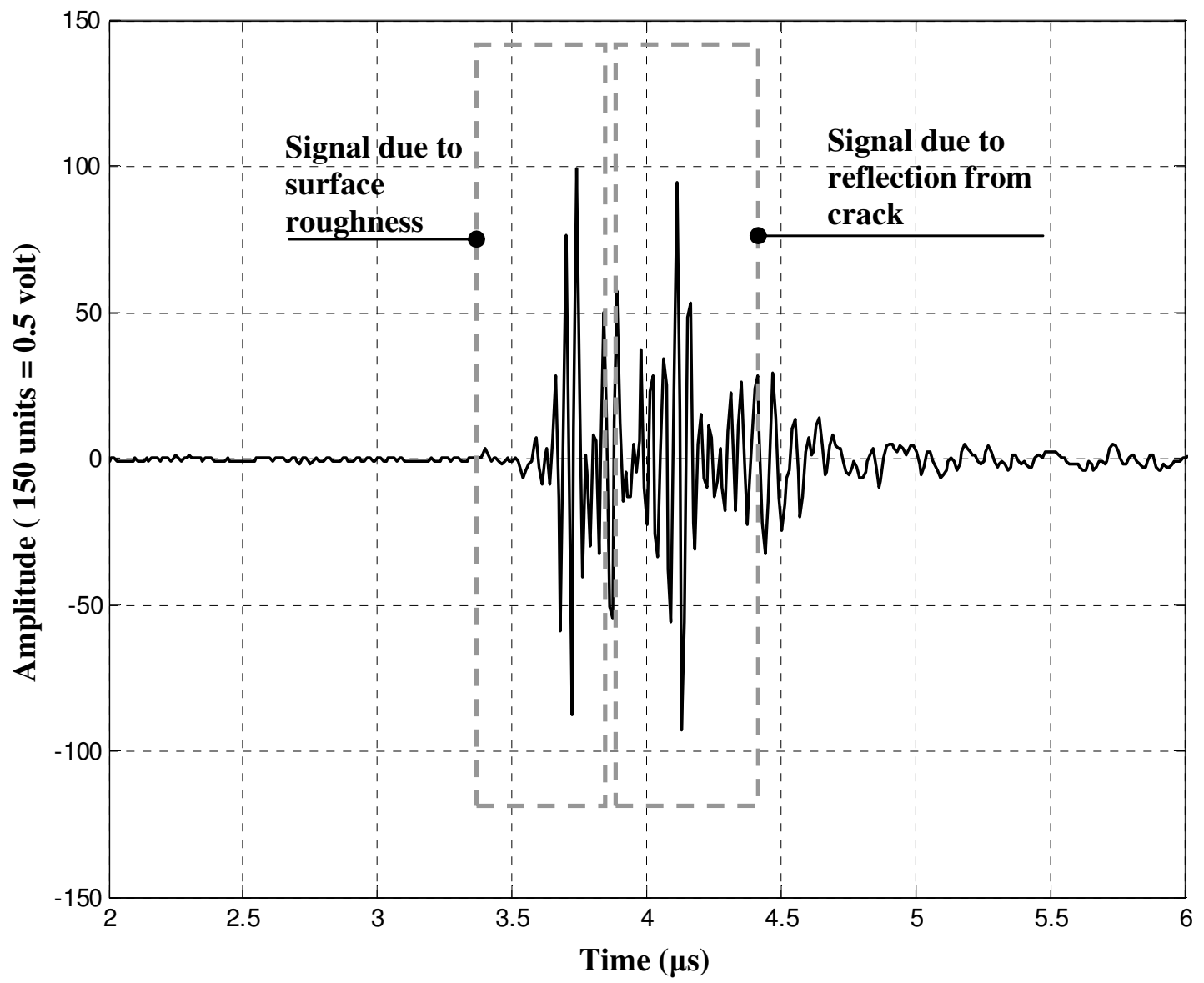

Fig. 3.5 Typical time-domain signal for the TAMU-4 specimen when the incident ultrasonic beam insonifies a crack through a rough surface. 


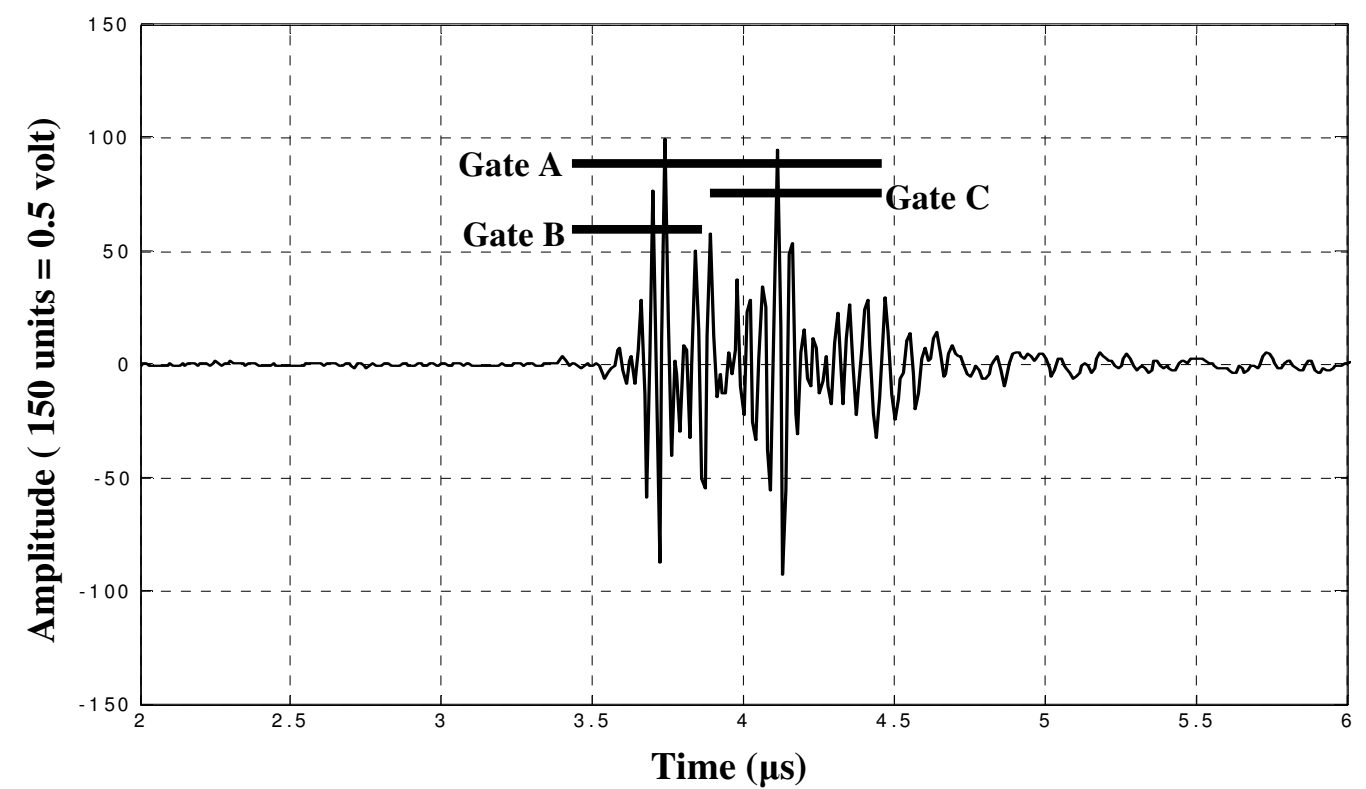

(a) Time domain signal, obtained when the interrogating acoustic beam insonifies a crack through the rough surface, showing position of gates $\mathrm{A}, \mathrm{B}$ and $\mathrm{C}$

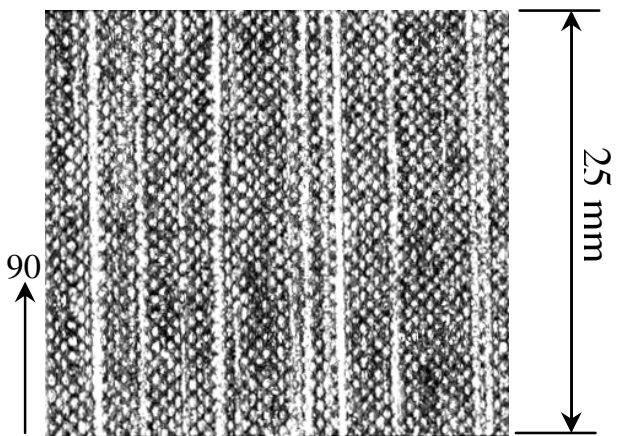

(b) Gate A

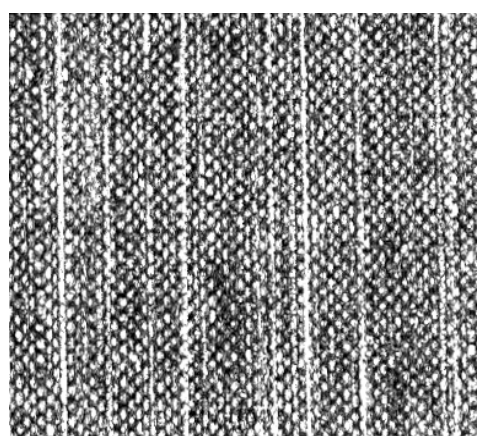

(c) Gate B

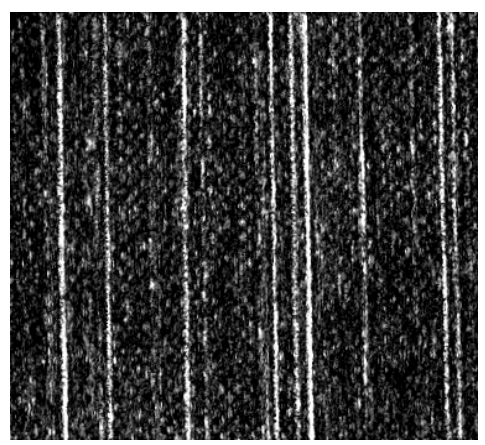

(d) Gate C

Fig. 3.6 Effect of gate positioning on image of cracks in the first ply of the TAMU-4 specimen. 
The image obtained by using this gate is shown in Fig. 3.6(c). Gate $\mathrm{C}$ is adjusted in such a way as to exclude most of the signal due to surface roughness. The resulting image is seen in Fig. 3.6(d). Cracks are observed only in the image corresponding to Gate C.

For the TAMU-2 and the TAMU-3 specimens it was observed that the presence of liners did not significantly alter the nature of the received signal during the ply-by-ply detection of cracks through the liners. The angle of incidence and the height of the transducer above the specimen were maintained the same as in the earlier case when the specimens were scanned for cracks through the rough side. For the TAMU-2 specimen, a typical time domain signal received in the presence of a crack in the ply adjacent to the aluminum liner while scanning through the liner, is shown in Fig. 3.7(a). In this case, to gather data, three gates of different lengths and at different positions were used; namely, Gate A of $1.8 \mu$ s duration covering the entire time domain signal, Gate B of $800 \mathrm{~ns}$ duration beginning approximately from the end of gate B. These gates are shown in Fig. 3.7(a). The resulting images using Gates A, B and C are presented in Fig. 3.7(b), Fig. 3.7(c) and Fig. 3.7(d) respectively. From these images, it is clear that the position of a gate over the time domain signal did not greatly influence the resulting images.

Ply-by-ply ultrasonic NDE results for all the eight plies of the TAMU-2 and the TAMU-3 specimens are presented in Figs. 3.8(a) through (h) and Figs. 3.9(a) through (h) respectively. The liner is on the first ply for both the specimens. Figures 3.10 (a) through (h) show the cracks in all the eight plies of the TAMU-4 specimen. As done earlier in the case of TAMU-1 specimen, in the spirit of self-calibration, the cracks in the $-45_{2}$ plygroup of all the three specimens were detected using two different acoustic paths, one 


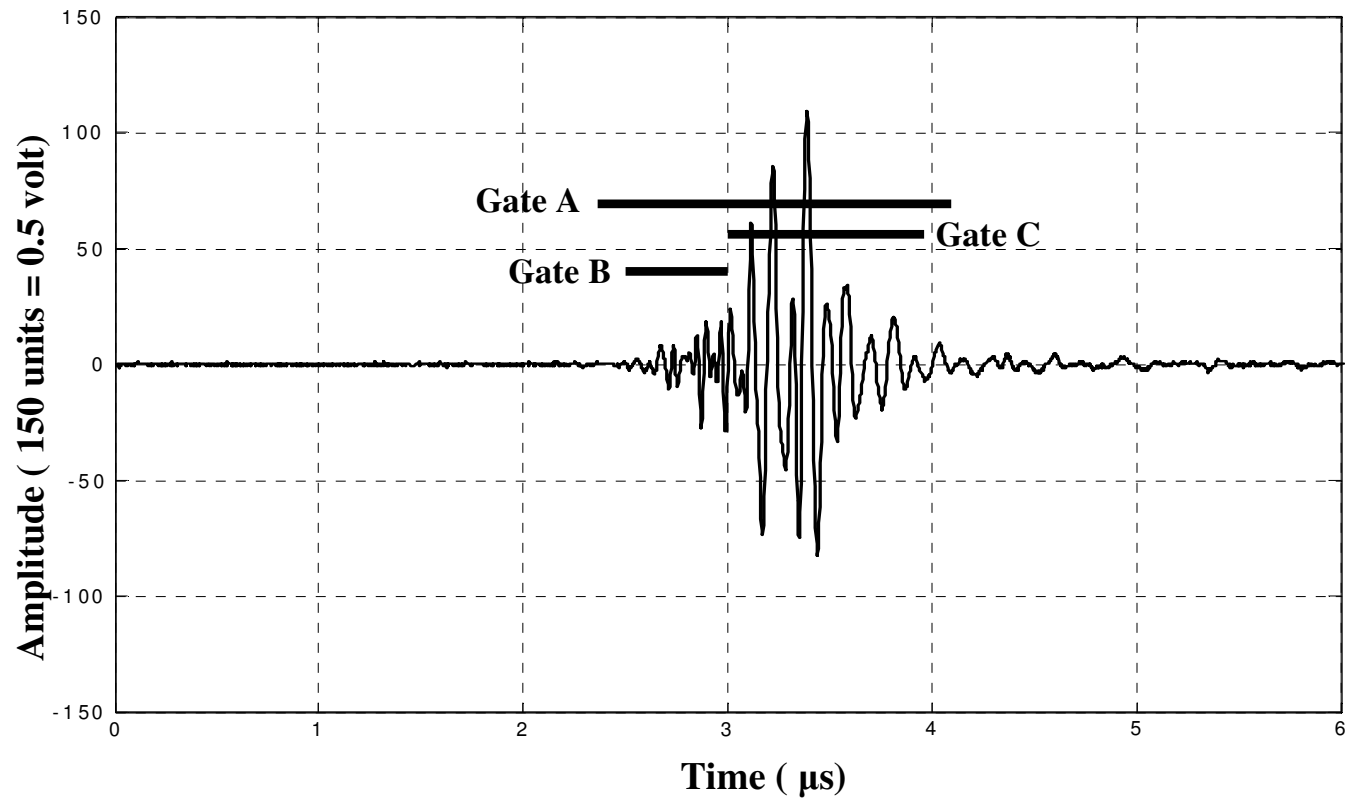

(a) Time domain signal, obtained when the interrogating acoustic beam insonifies a crack through the aluminum liner, showing position of gates A, B and C

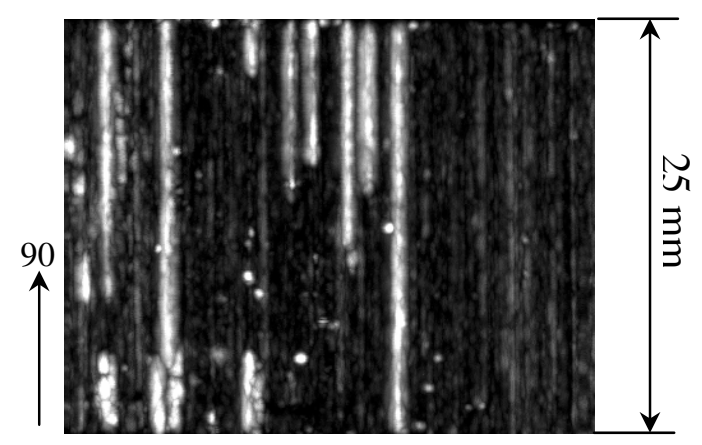

(b) Gate A

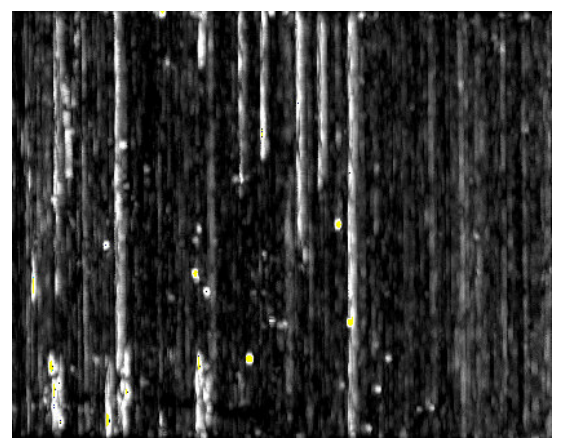

(c) Gate B

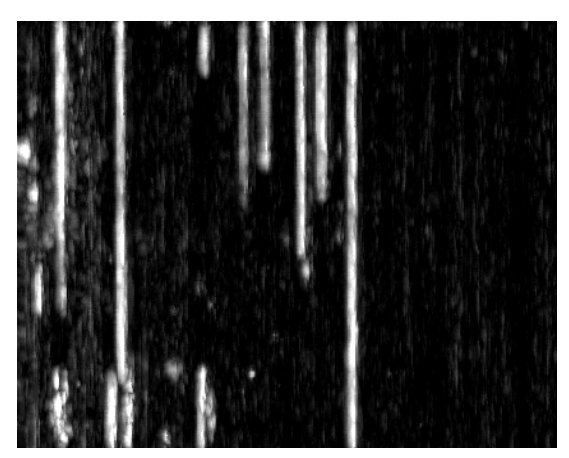

(d)Gate C

Fig. 3.7 Effect of gate positioning on image of cracks in the first ply (ply adjacent to the aluminum liner) of the TAMU-2 specimen. 


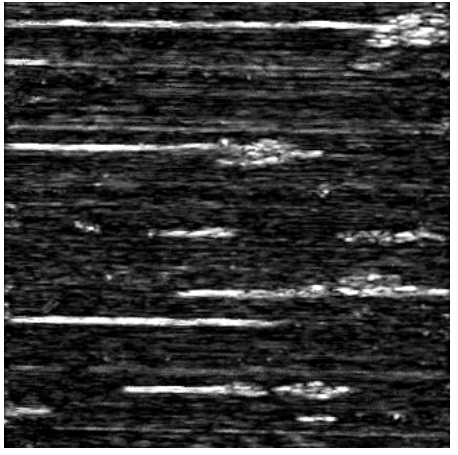

(a) First ply $\left(90^{\circ}\right)$

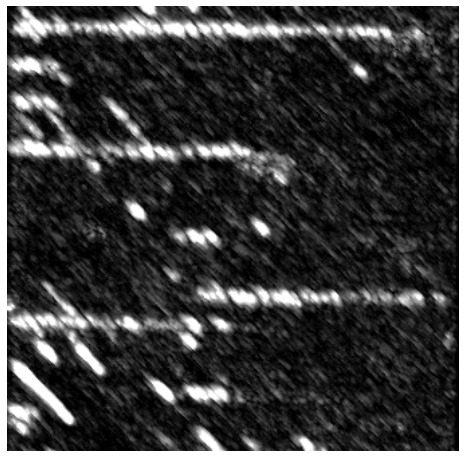

(b) Second ply $\left(45^{\circ}\right)$

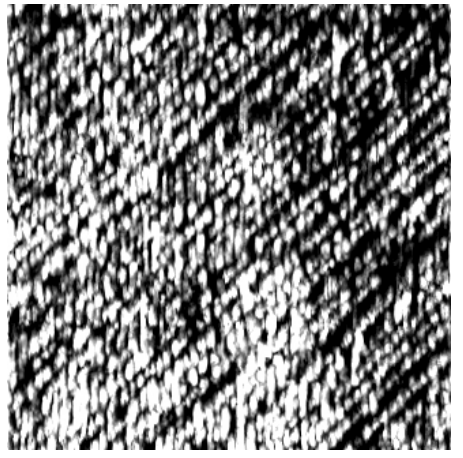

(c) Third ply $\left(0^{\circ}\right)$

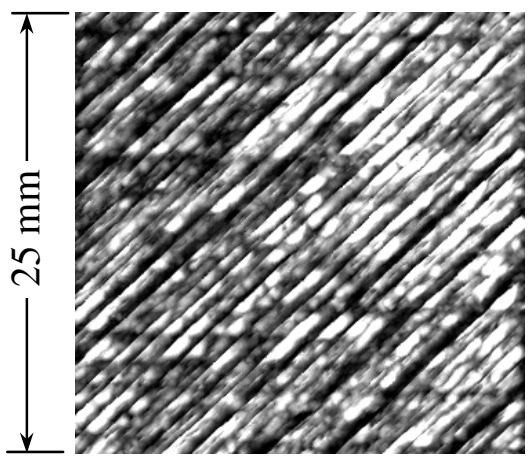

(d) Fourth + Fifth ply group $\left(-45^{\circ}\right)$
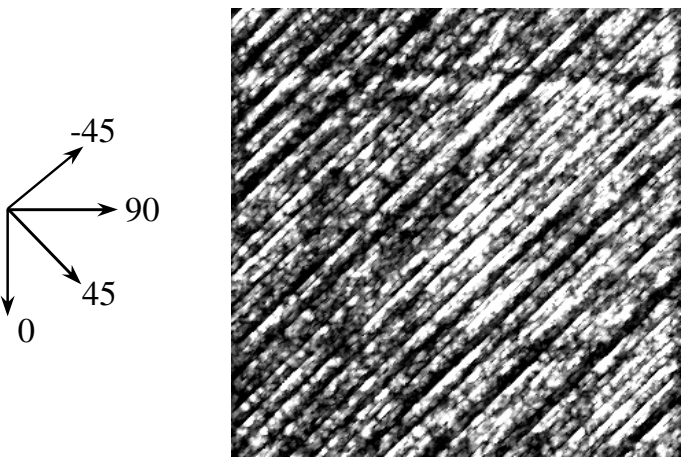

(e) Fourth + Fifth ply group from other side

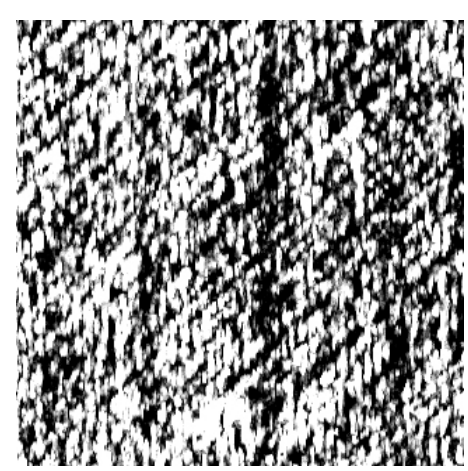

(f) Sixth ply $\left(0^{\circ}\right)$

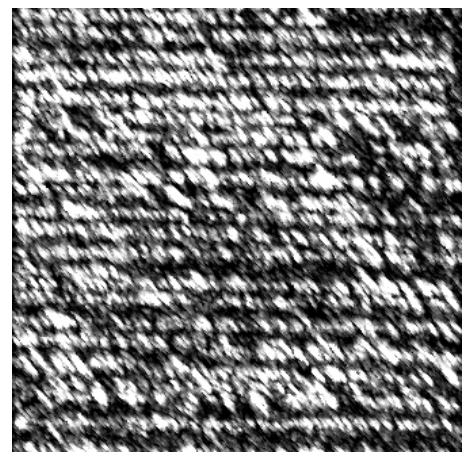

(g) Seventh ply $\left(45^{\circ}\right)$

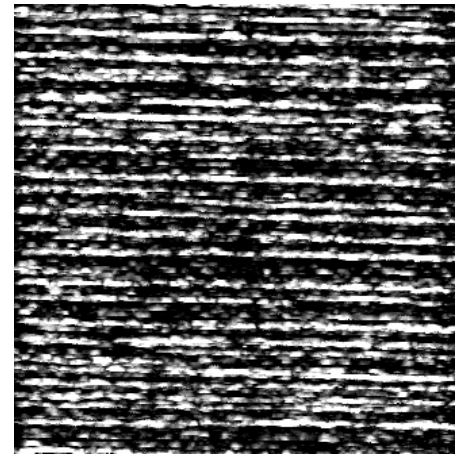

(h) Eighth ply $\left(90^{\circ}\right)$

Fig. 3.8 Ultrasonic images of cracks in the eight plies of the [90/45/0/-45] TAMU-2 specimen. $\left(\right.$ Angle of incidence $=25^{\circ}$ ) 


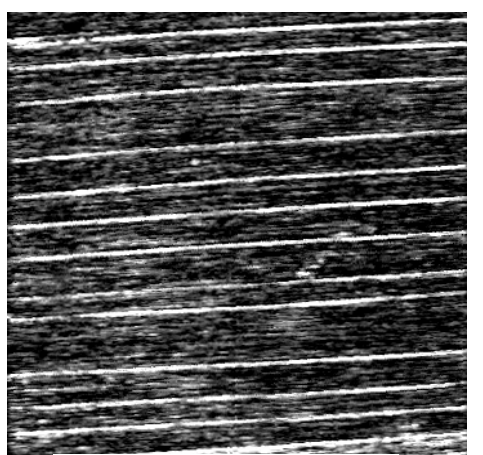

(a) First ply $\left(90^{\circ}\right)$

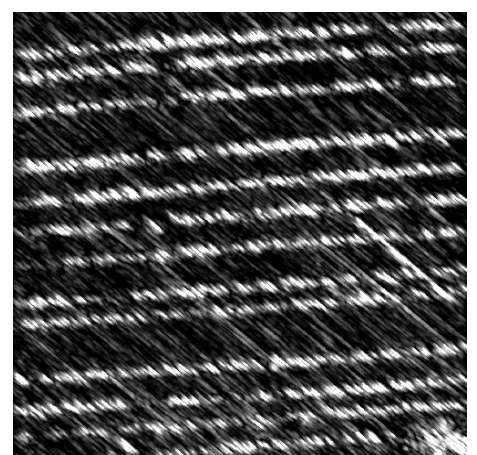

(b) Second ply $\left(45^{\circ}\right)$

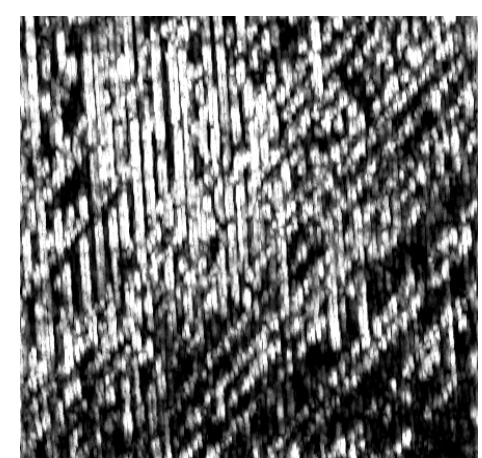

(c) Third ply $\left(0^{\circ}\right)$

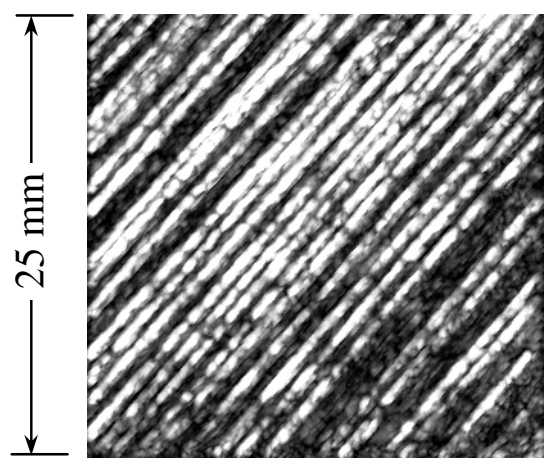

(d) Fourth + Fifth ply group $\left(-45^{\circ}\right)$
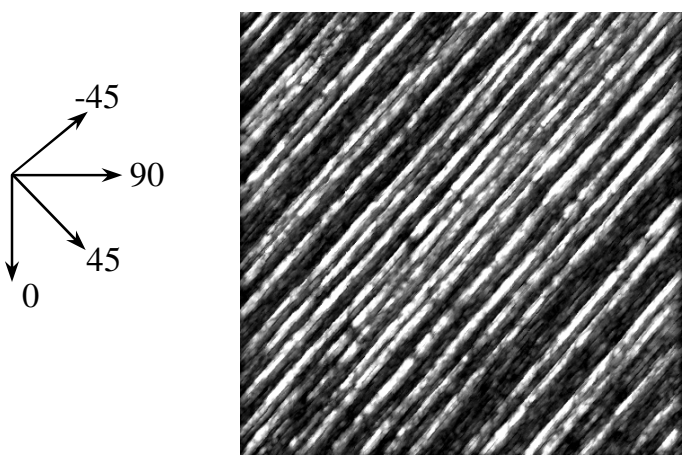

(e) Fourth + Fifth ply group from other side

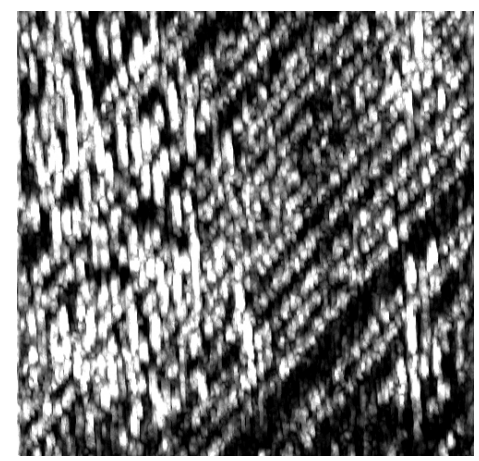

(f) Sixth ply $\left(0^{\circ}\right)$

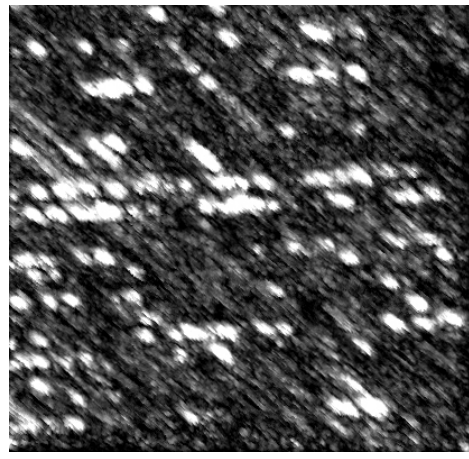

(g) Seventh ply $\left(45^{\circ}\right)$

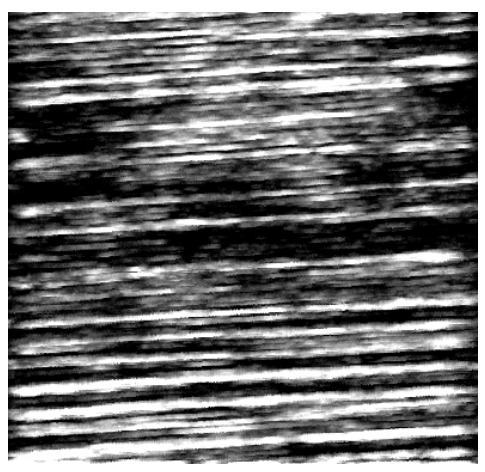

(h) Eighth ply $\left(90^{\circ}\right)$

Fig. 3.9 Ultrasonic images of cracks in the eight plies of the [90/45/0/-45] s

TAMU-3 specimen. $\left(\right.$ Angle of incidence $=25^{\circ}$ ) 


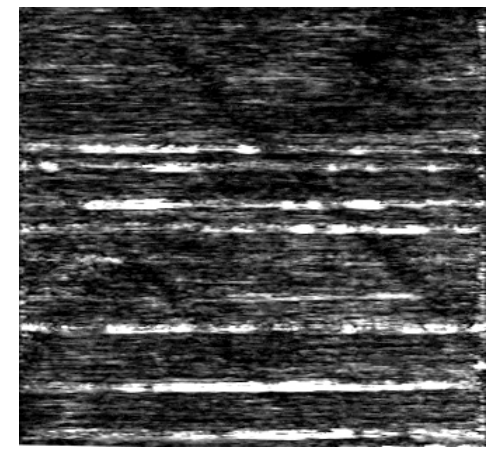

(a) First ply $\left(90^{\circ}\right)$

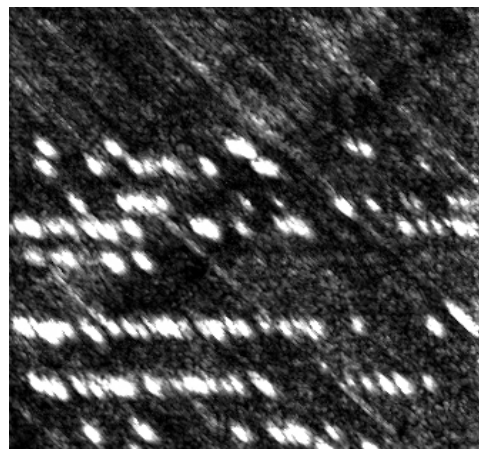

(b) Second ply $\left(45^{\circ}\right)$

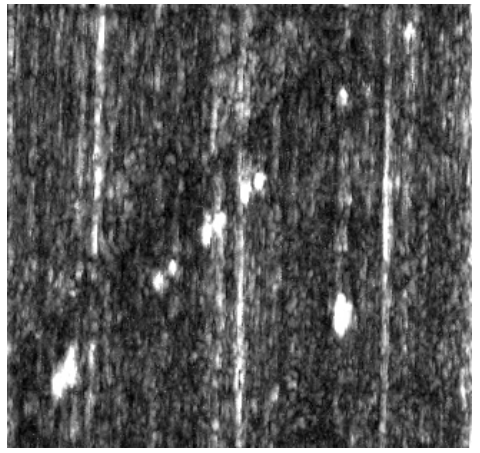

(c) Third ply $\left(0^{\circ}\right)$

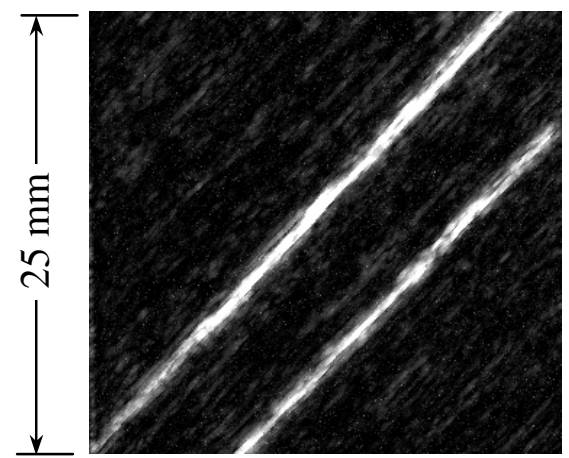

(d) Fourth + Fifth ply group $\left(-45^{\circ}\right)$
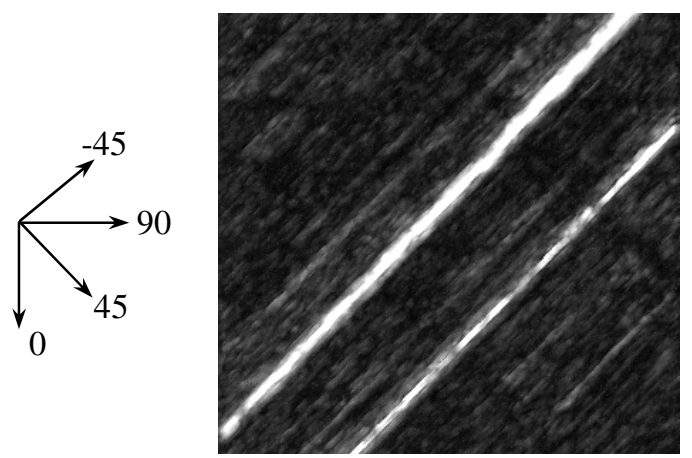

(e) Fourth + Fifth ply group from other side

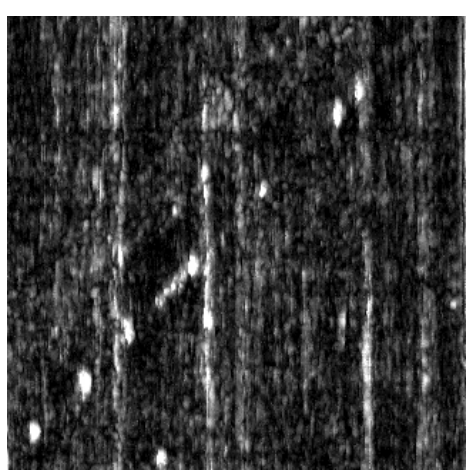

(f) Sixth ply $\left(0^{\circ}\right)$

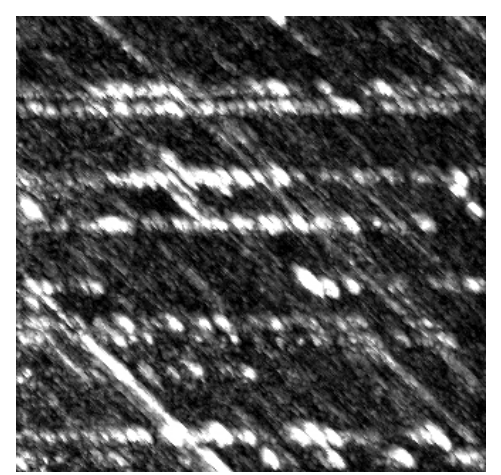

(g) Seventh ply $\left(45^{\circ}\right)$

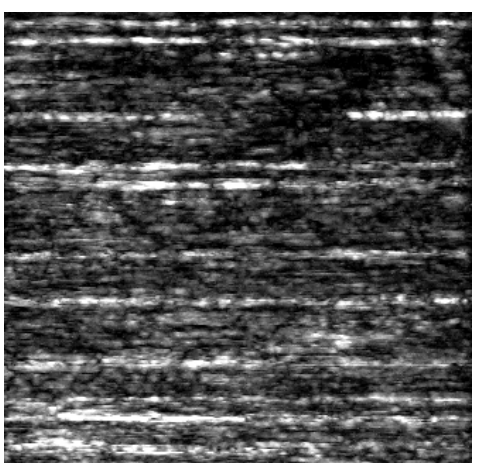

(h) Eighth ply $\left(90^{\circ}\right)$

Fig. 3.10 Ultrasonic images of cracks in the eight plies of the [90/45/0/-45]s TAMU-4 specimen. $\left(\right.$ Angle of incidence $=25^{\circ}$ ) 
from either side of the specimen. In these specimens too, short cracks were observed in the second, third, sixth and the seventh plies. The same arguments, as put forward for the TAMU-1 specimen, are valid for the short cracks in these specimens.

In order to further ascertain the presence of short cracks a destructive test of the TAMU-2 specimen was undertaken. Details of this exercise are explained next. For greater clarity and ease of comparison, the optical microscopy results during the destructive test were compared with the X-ray image of the specimen instead of an ultrasonic image. The X-ray image is presented in Fig. 3.11(a). The laminate was first sectioned along the line a-a as shown in Fig. 3.11(b), polished and examined optically at 200X magnification for cracks in the second ply $\left(45^{\circ}\right)$. As expected, the examination of the section along a-a did not reveal any cracks in the second ply. Subsequently, the sample was carefully sectioned along the lines b-b, c-c, d-d and optical examination was repeated in each case. In c-c, some cracks in the second ply appeared. Their positions are presented in Fig. 3.11(b) as tick-marks in the $45^{\circ}$ directions. Note that the positions of the optically observed cracks coincides with the positions of dark lines in the x-ray image and when stitch cracks are not seen in the x-ray image they are also not observed in the optical examination. This serial sectioning confirmed the presence of short cracks in the second ply of the TAMU-3 laminate. Since TAMU-1, TAMU-2 and TAMU-4 specimens showed similar damage characteristics as shown by the TAMU-2 specimen, the corroboration of the presence of short cracks in the TAMU-2 essentially confirms the presence of short cracks in the other specimens. 


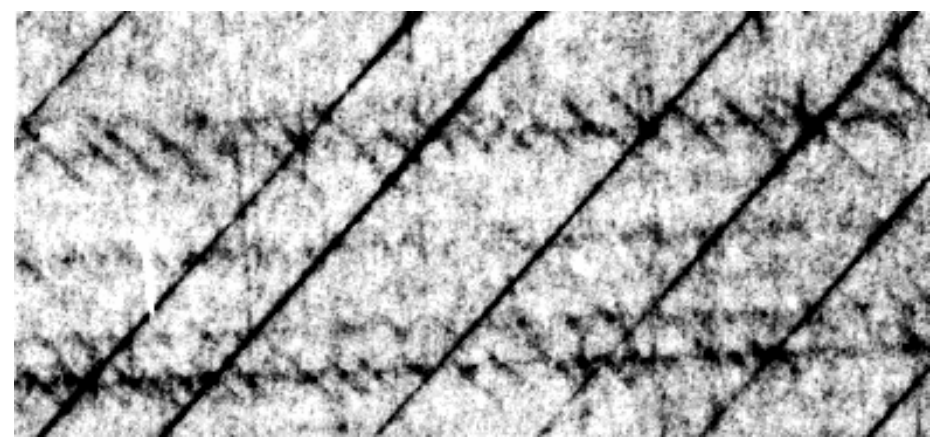

(a)

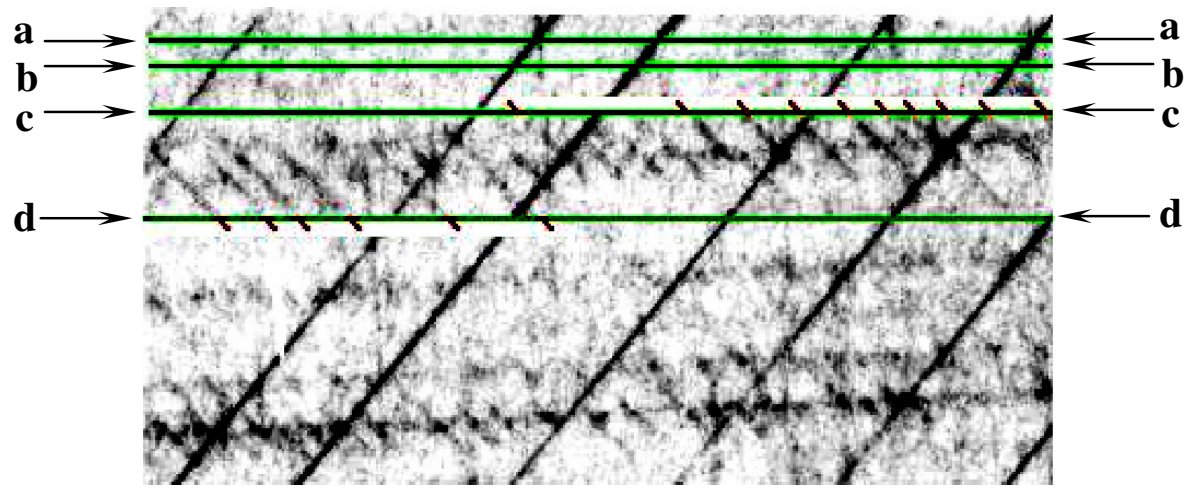

(b)

Fourth ply
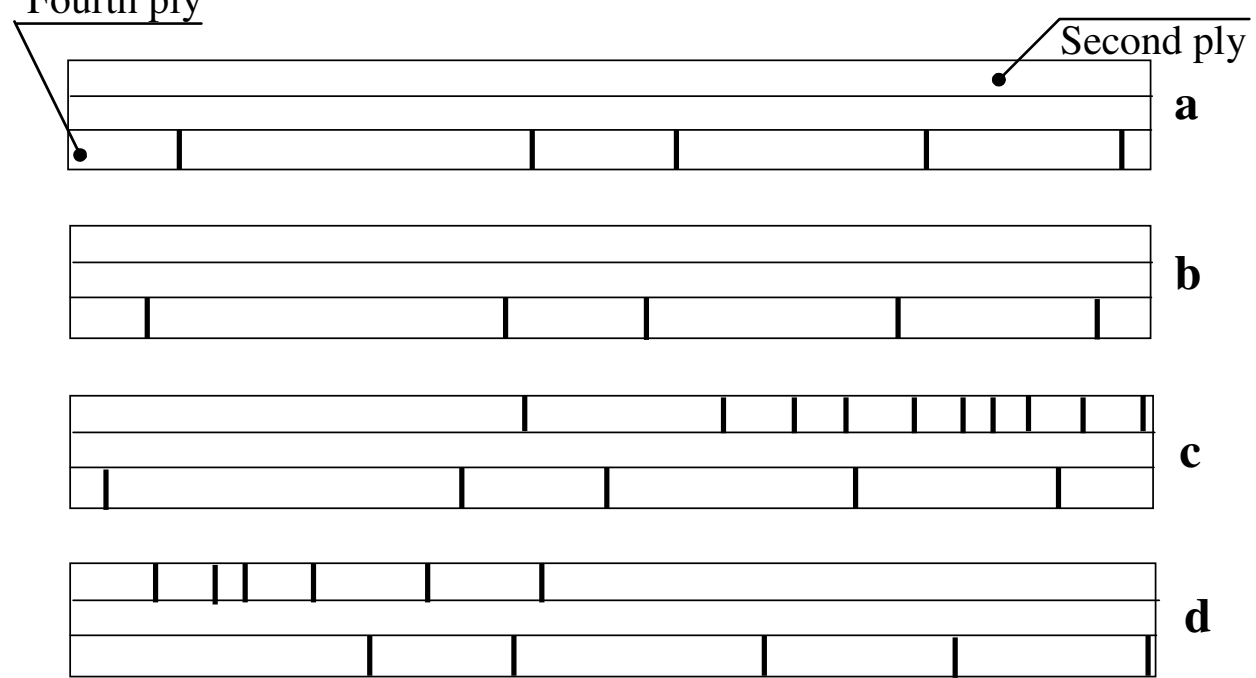

d

(c)

Fig. 3.11 (a) X-ray image showing short cracks in the second ply $\left(45^{\circ}\right)$ of the TAMU-2 specimen, (b) comparison of the results of serial sectioning with the $\mathrm{x}$-ray image, and (c) schematic of the optically detected edge crack positions in the second ply. 


\subsection{TAMU-5 Specimen}

This 18-ply [0/45/90/-45/0/45/90/-45/0] laminate was scanned for matrix cracks by a $20 \mathrm{MHz}$ transducer. A ply-by-ply examination of the laminate was conducted with angle of incidence set to $25^{\circ}$. A gate of $400 \mathrm{~ns}$ duration from the start of the received signal was used for all the plies. Cracks were found in only the first ply $\left(0^{\circ}\right)$, the second ply $\left(45^{\circ}\right)$ third ply $\left(90^{\circ}\right)$ and the fourth ply $\left(-45^{\circ}\right)$ orientation. Figures $3.12(a)$ through (d) show the matrix cracks in the first, second, third and the fourth plies respectively. It can be observed from a comparison of Fig. 3.12(a) with 3.12(b), that the short cracks in the second ply $\left(45^{\circ}\right)$ are aligned along the long cracks in the sparsely damaged first ply $\left(0^{\circ}\right)$. From Fig. 3.12(c) and Fig. 3.12(d) it is clear that most of the matrix cracks in the third and the fourth plies are either internal or partial cracks. It is noted that, in certain areas in Fig. 3.12, the short cracks in the third ply are driven by cracks in the fourth ply, whereas in other portions the short cracks in the fourth ply are driven by cracks in the third ply. It is also interesting to note that though the arrangement of cracks in the third ply greatly influenced the pattern of crack formation in the fourth ply, it did not have any influence on the crack pattern in the second ply.

The results obtained by using X-radiography for the same area as shown in Fig. 3.12 have been presented earlier in Fig. 2.32. It is interesting to note that, X-radiography did not yield any information about the cracks in any of the four damaged plies. Also, from the schematics of the optically detected cracks in Figs. 3.12(c) and 3.12(d), it is clear that optical microscopy could detect only a small number of cracks visible at one of 

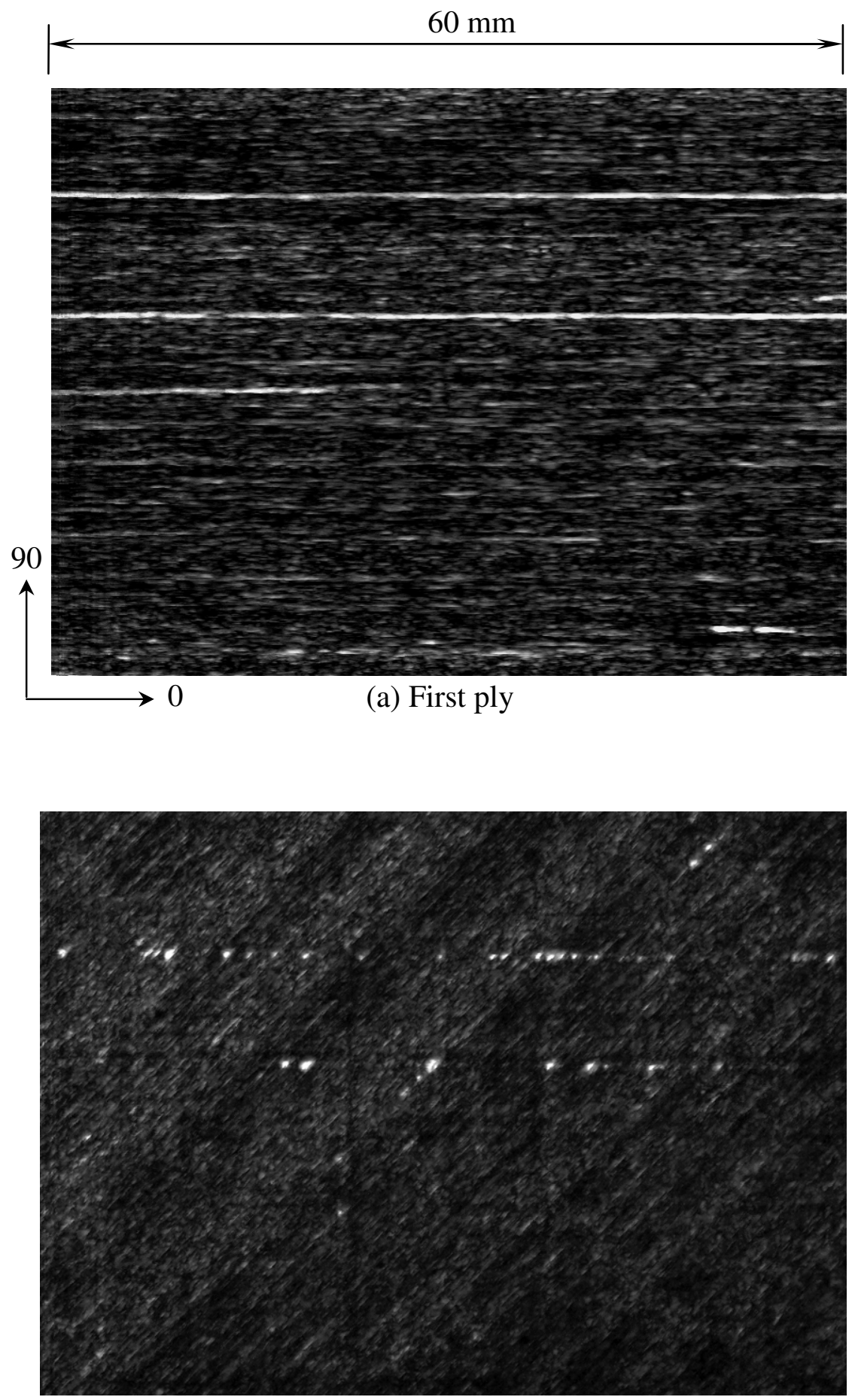

(a) Second ply

Fig. 3.12 Ultrasonic images of matrix cracks in the TAMU-5 specimen. Cracks in the (a) first ply, (b) second ply. 


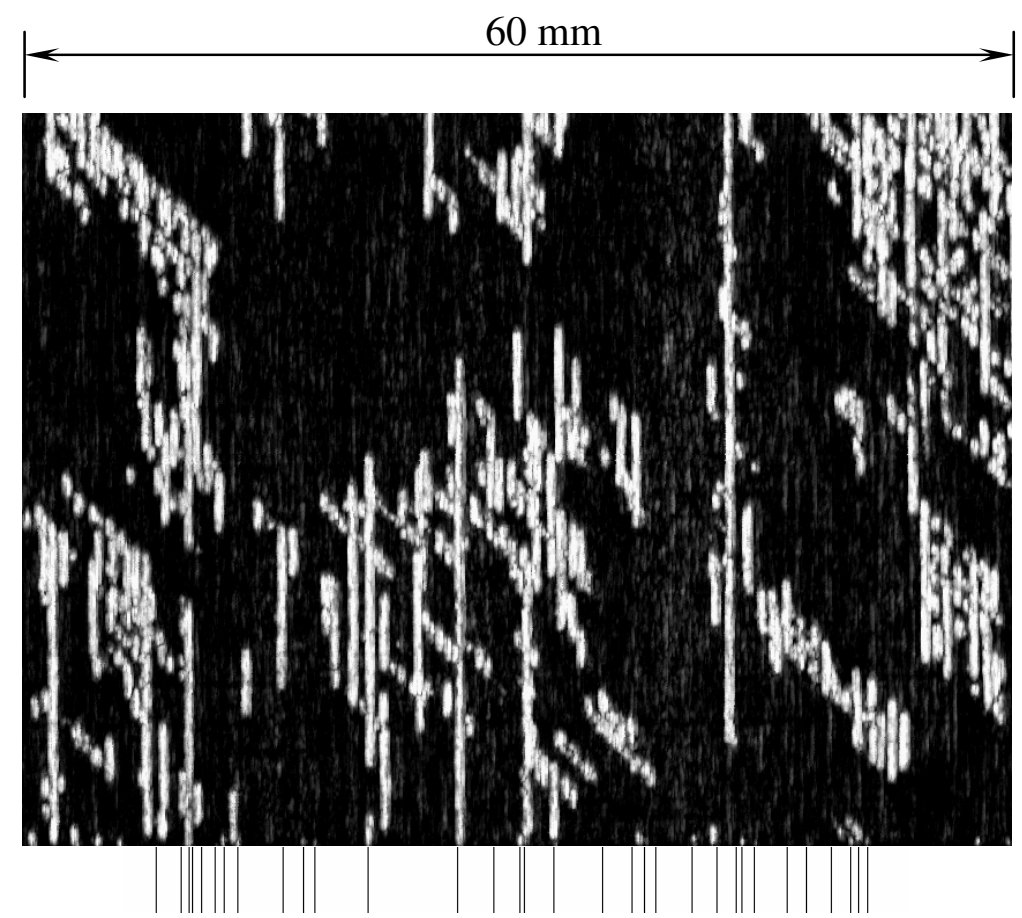

(c) Third ply

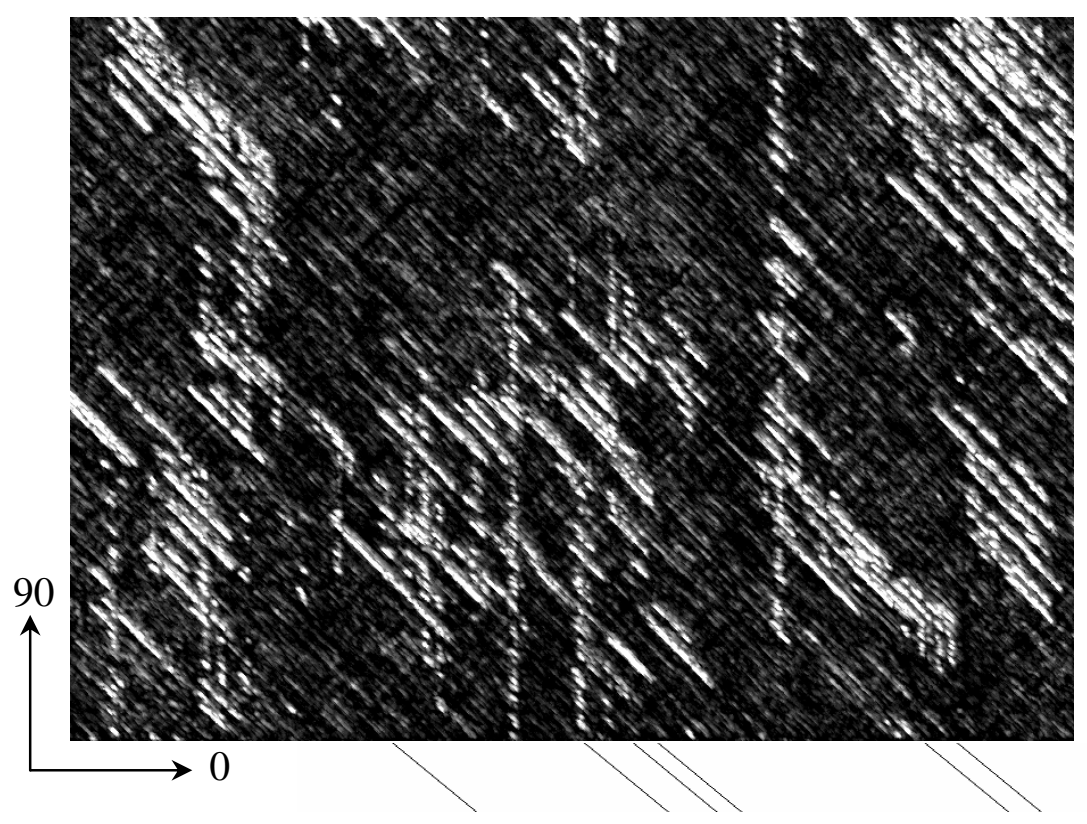

(d) Fourth ply

Fig. 3.12 (continued) Cracks in the (c) third ply and (d) fourth ply of the TAMU-5 specimen. (Schematic of the optically detected cracks is shown at the bottom of the images) 
the edges. This case is a glorious example demonstrating the advantages the ultrasonic ply-by-ply crack detection technique over X-radiography and optical microscopy.

For the sake of clarity of communication, in the following, the first through fourth plies in the above discussion will be referred to as Ply \#1 through Ply \#4 respectively. In another experiment, the specimen was flipped over and again scanned for matrix cracks in the Ply \#1 through Ply \#4 from the other side. Obviously, after flipping over, the interrogating acoustic beam has to travel through 17 plies in order to detect the cracks in Ply \#1 $\left(0^{\circ}\right)$; through 16 plies to detect the cracks in the Ply \#2 $\left(90^{\circ}\right)$ and so on. This is schematically shown in Figs. 3.13 and 3.14. Though, a weaker reflected field was observed than in the previous case, the cracks in the two plies could still be detected. The signal to noise ratio was found to be about $15 \mathrm{~dB}$. The resulting images of cracks in Ply \#3 and Ply \#4 are shown in Fig. 3.13(a) and Fig. 3.14(a) respectively. For the purpose of comparison, the corresponding images obtained earlier, when the acoustic beam traveled through two plies for Ply \#3 (Fig. 3.12(c)) and through three plies for Ply \#4 (Fig. 3.12(d)), are reproduced in Fig. 3.13(b) and Fig. 3.14(b) respectively. Comparing the areas marked by dotted rectangles in Figs. 3.13(a) and 3.14(a) with 3.13(b) and 3.14(b) respectively, it is seen that the cracks in the marked area in the Ply \#3 and Ply \#4 were not detected after flipping. This was because, after flipping, the presence of a strain gauge on the top-most ply blocks the interrogating acoustic beam from reaching the interior plies. A photograph of the corresponding area of the TAMU-5 specimen (again marked by a dotted rectangle), showing the strain gauge, is presented in Fig. 3.15. 

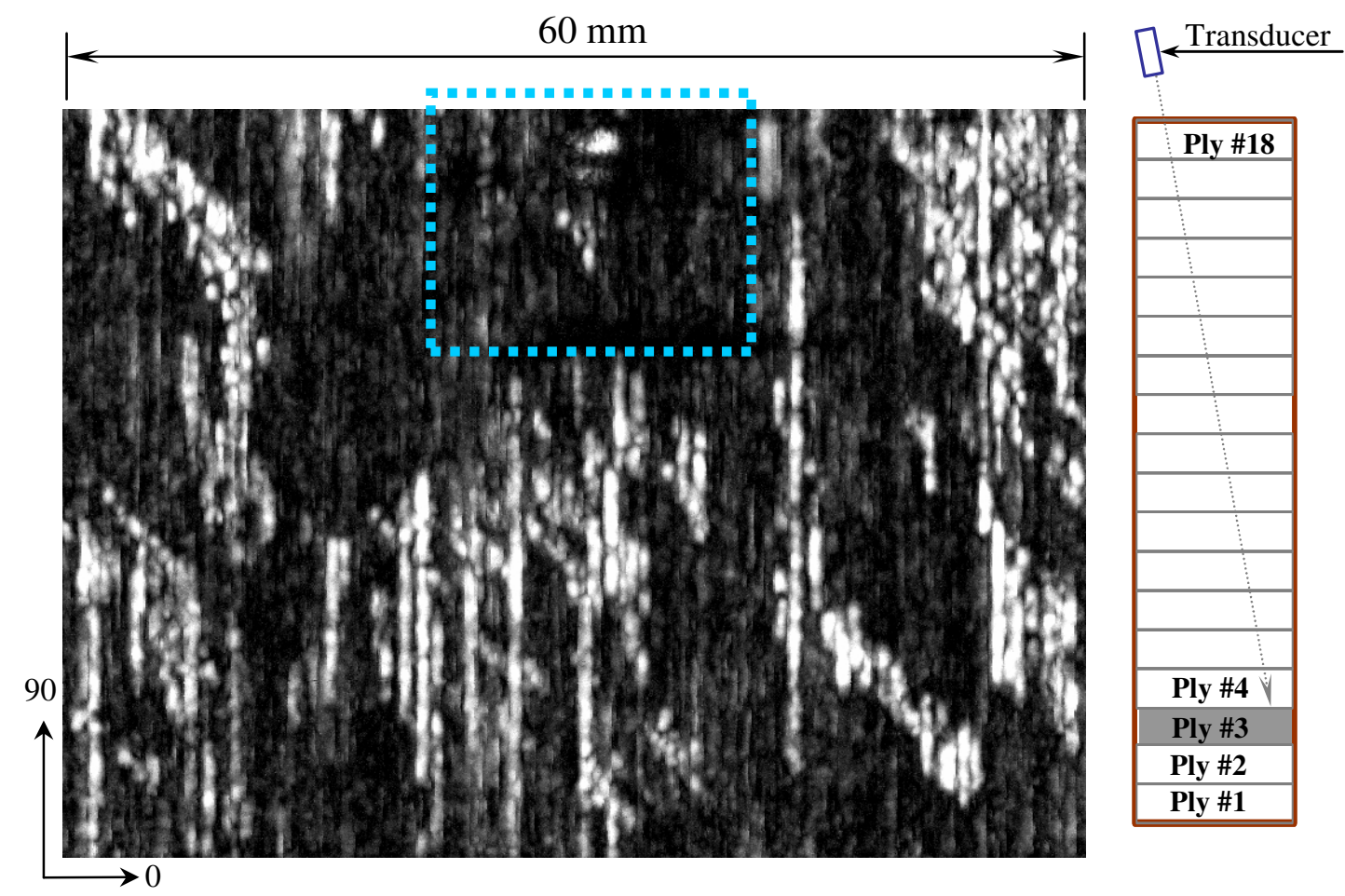

(a) Cracks in the third ply as detected through fifteen plies
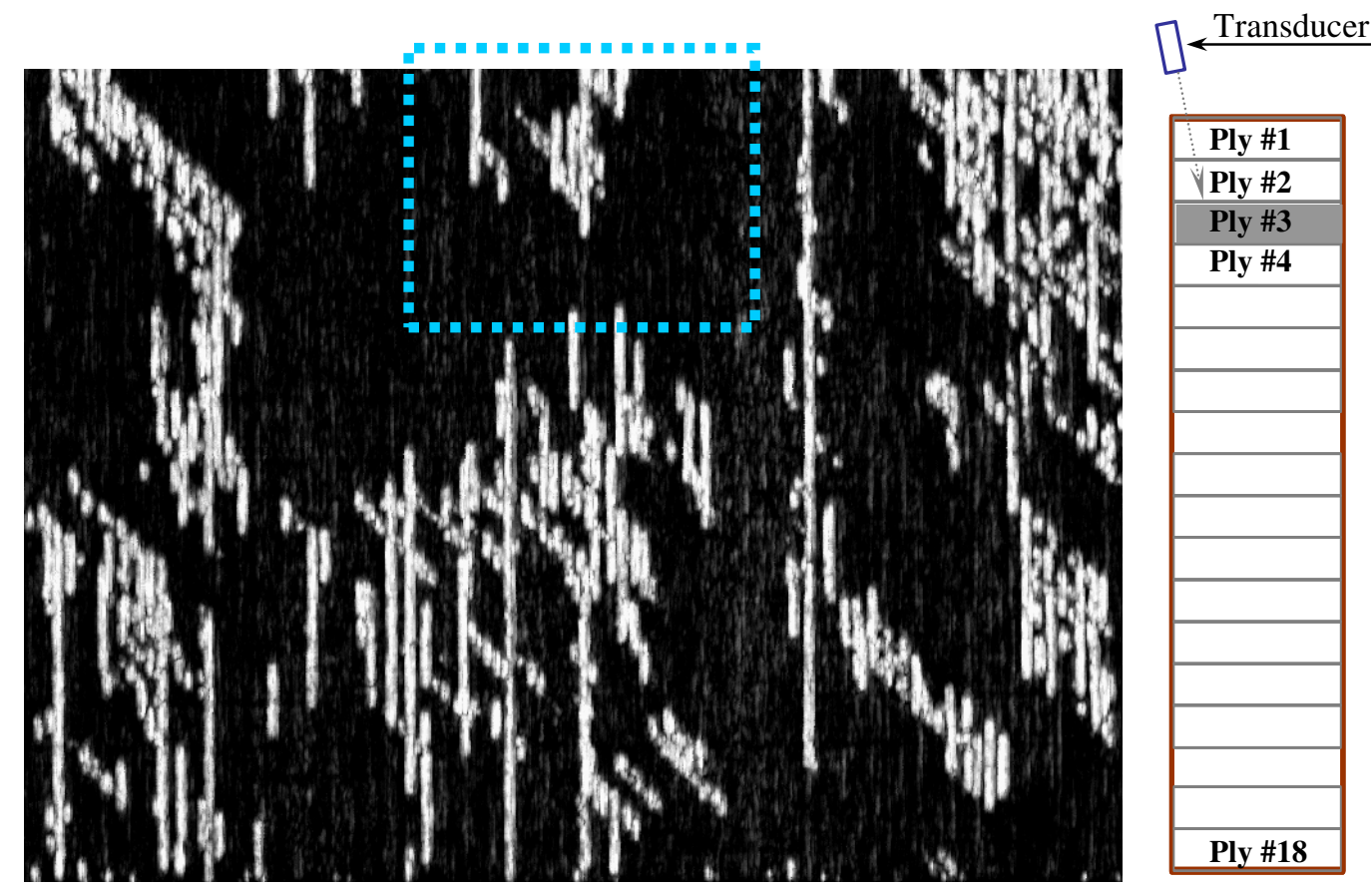

(b) Cracks in third ply as detected through two plies

Fig. 3.13 Ultrasonic image of matrix cracks in the third ply of the TAMU-5 specimen obtained by using two different acoustic paths. ( Angle of incidence $=25^{\circ}$ ) 

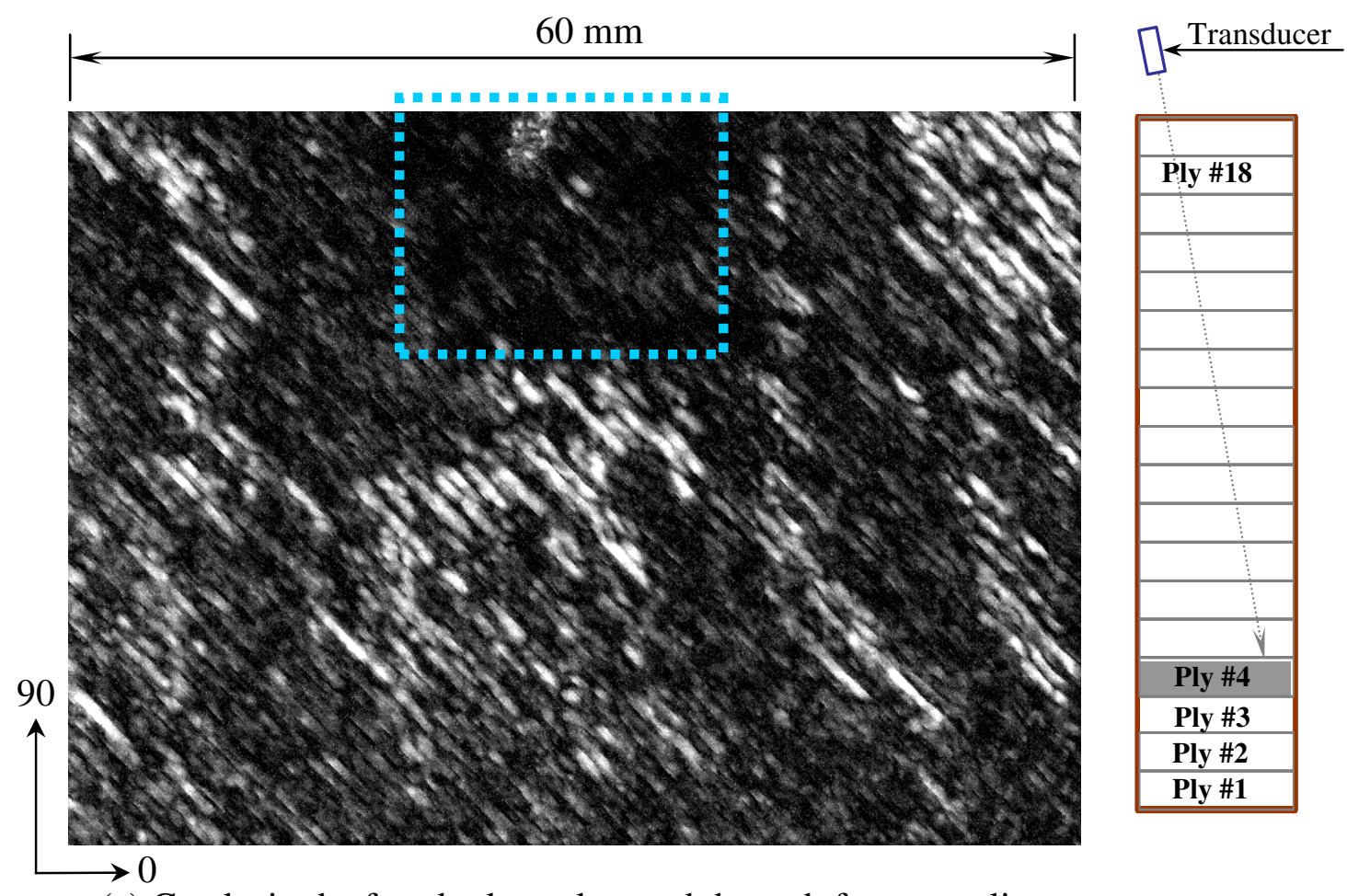

(a) Cracks in the fourth ply as detected through fourteen plies
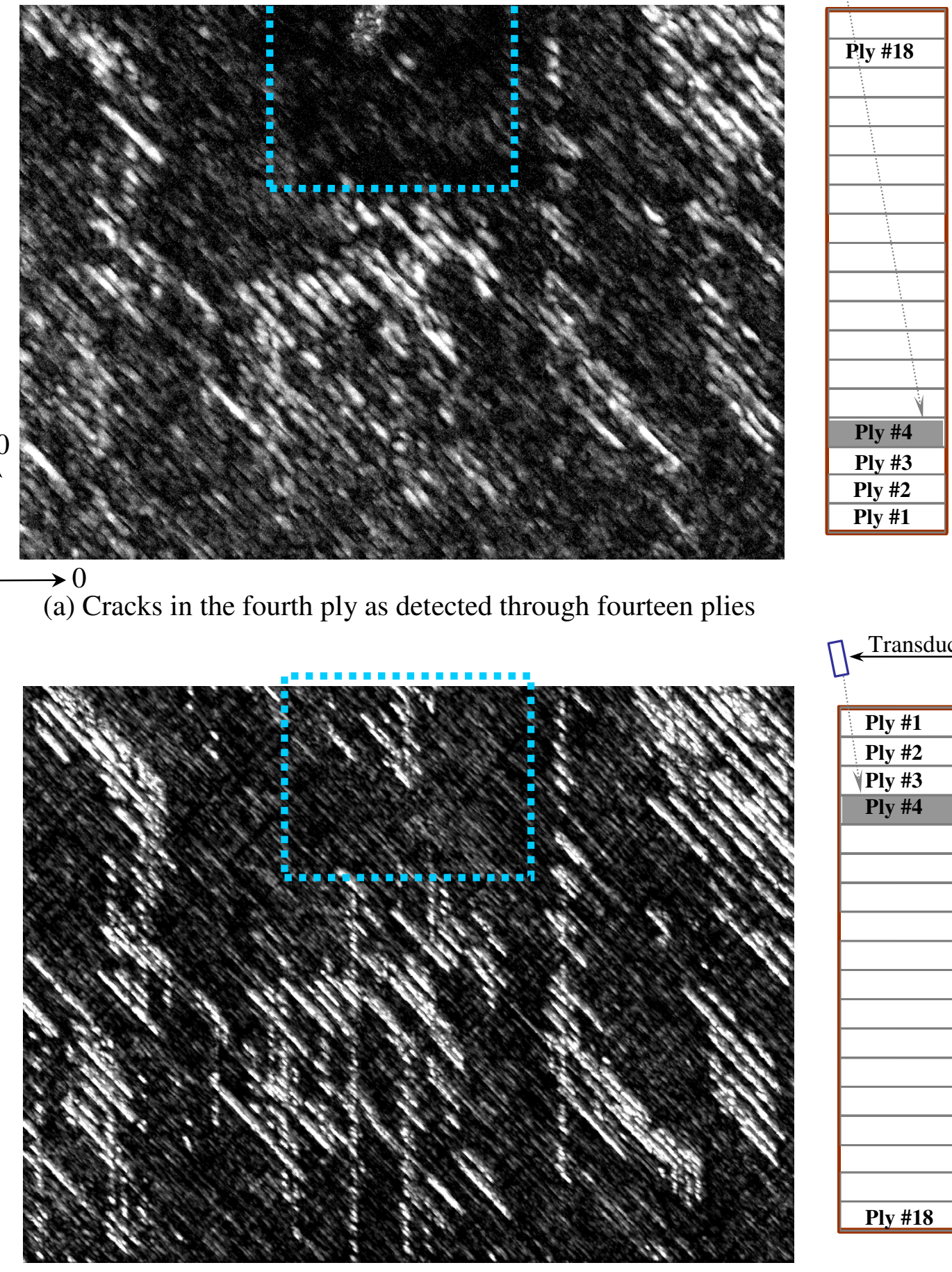

[ $\stackrel{\text { Transducer }}{\longleftarrow}$

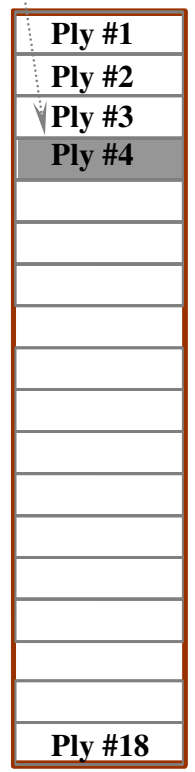

(b) Cracks in the fourth ply as detected through three plies

Fig. 3.14 Ultrasonic image of matrix cracks in the fourth ply of the TAMU-5 specimen obtained by using two different acoustic paths. ( Angle of incidence $=25^{\circ}$ ) 


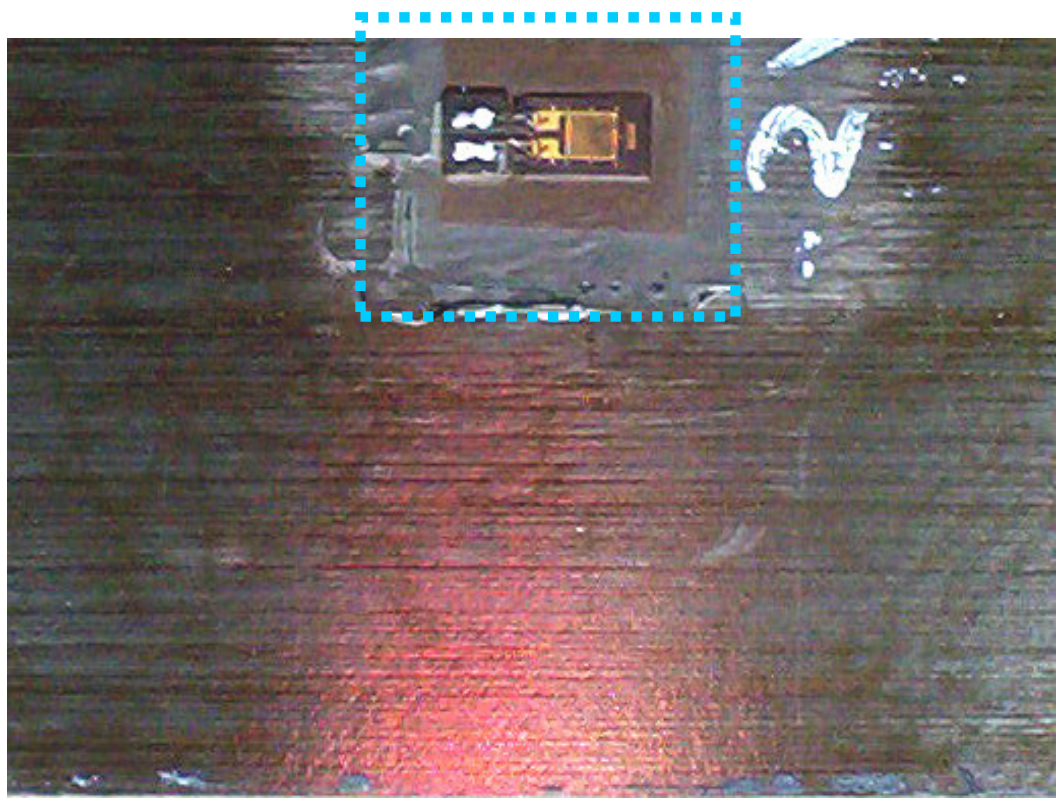

Fig. 3.15 A photograph of the TAMU-5 specimen showing the strain gage. 


\section{CHAPTER IV}

\section{CONCLUSIONS}

An ultrasonic technique has been developed to detect matrix cracks in each of the plies of laminated composites even in the presence of significant damage in all of the plies. The ultrasonic data has been thoroughly corroborated by calibration with wellestablished techniques like X-ray radiography and optical microscopy. The technique has been successfully applied for the detection of a high density of matrix cracks in cross-ply as well as in quasi-isotropic composite laminates. Results obtained by the variation of three major experimental parameters, namely, the azimuthal angle $(\beta)$, the angle of incidence $(\alpha)$ and the vertical distance between the transducer and the specimen show that the ultrasonic polar backscattering technique offers substantial flexibility in the adjustment of these parameters for the ply-by-ply detection of matrix cracks. The calibration experiments conducted are strongly suggestive of the presence of reflected waves. However, an exhaustive theoretical analysis of the complicated nature of wave propagation in laminated composites has to be undertaken in order to conclusively establish the presence of a reflected field. 


\section{REFERENCES}

1. Freeman, D.C. Jr., Talay, T. A., and Austin, R. E., 1997, "Reusable Launch Vehicle Technology Program,” Acta Astronautica, 41(11), pp. 777-790.

2. Powell, R.W., Lockwood, M. K., and Cook, S. A., 1998, "The Road from the NASA Access-to-Space Study to a Reusable Launch Vehicle," $49^{\text {th }}$ International Astronautical Congress, Melbourne, Australia.

3. Rivers, H. K., and Sikora, J. G., 2001, "Detection of Micro-Leaks through Complex Geometries under Mechanical Load and at Cryogenic Temperature," $42^{\text {nd }}$ AIAA/ASME/ASCE/AHS/ASC Structures, Structural Dynamics, and Materials Conference Proceedings, Seattle, Washington.

4. Aoki, T., Ishikawa, T., Kumazawa, H., and Morino, Y., 2000, "Mechanical Performance of CF/Polymer Composite Laminates under Cryogenic Conditions," $41^{\text {st }}$ AIAA/ASME/ASCE/AHS/ASC Structures, Structural Dynamics, and Materials Conference Proceedings, Atlanta, Georgia.

5. Final Report of the X-33 Liquid Hydrogen Tank Test Investigation Team, May 2000, NASA George C. Marshall Space Flight Center, Huntsville, Alabama.

6. McManus, H., Uebelhart, S., and Faust, A., 2001, "Gas Permeability of Thermally Cycled Graphite-epoxy Composites," $16^{\text {th }}$ Annual Technical Conference Proceedings, American Society for Composites, Blacksburg, Virginia. 
7. Kumazawa, H., Aoki, T., Ishikawa, T., and Susuki, I., 2001, "Modeling of Propellant Leakage Through Matrix Cracks in Composite Laminates," $42^{\text {nd }}$ AIAA/ASME/ASCE/AHS/ASC Structures, Structural Dynamics, and Materials Conference Proceedings, Seattle, Washington.

8. Reider, R., and Edeskuty, F. J., 1979, "Hydrogen Safety Problems," International Journal of Hydrogen Energy, 4, pp. 41-45.

9. Hansel, J. G., Mattern, G. W., and Miller, R. N., 1993, "Safety Considerations in the Design of Hydrogen-powered Vehicles," International Journal of Hydrogen Energy, 18(9), pp. 783-790.

10. Maslov, K., Kim, R., Kinra, V., and Pagano, N., 2000, “A New Technique for the Ultrasonic Detection of Internal Transverse Cracks in Carbon-fibre/bismaleimide Composite Laminates," Composites Science and Technology, 60, pp. 2185-2190.

11. Birt, E. A., 2000, "The Application of X-radiography to the Inspection of Composites," Insight, 42(3), pp. 151-157.

12. Krautkramer, J., and Krautkramer, H., 1969, Ultrasonic Testing of Materials, Springer-Verlag, New York.

13. Shull, P., 2002, Nondestructive Evaluation Theory, Techniques and Applications, Marcel Dekker, New York.

14. Wooh, S. C., and Daniel, I. M., 1990, "Enhancement Techniques for Ultrasonic Nondestructive Evaluation of Composite Materials," Journal of Engineering Materials and Technology, 112, pp. 175-182. 
15. Moran, T., Crane, R., and Andrews, R., 1985, "High-resolution Imaging of Microcracks in Composites," Materials Evaluation, 43, pp. 536-540.

16. Kaczmarek, H., 1993, "Ultrasonic Detection of the Development of Transverse Cracking under Monotonic Tensile Loading," Composites Science and Technology, 46, pp. 67-75.

17. Dayal, V., and Kinra, V. K., 1989, "Leaky Lamb Waves in Anisotropic Plate I: An Exact Solution and Experiments," Journal of Acoustical Society of America, 85(6), pp. 2268-2274.

18. Dayal, V., and Kinra, V. K., 1991, "Leaky Lamb Waves in Anisotropic Plate II: Nondestructive Evaluation of Matrix Cracks in Fiber-reinforced Composites," Journal of Acoustical Society of America, 89(4), pp. 1590-1598.

19. Bar-Cohen, Y., Lih, S., and Mal, A. K., 2001, "NDE of Composites Using Leaky Lamb Waves (LLW)," The e-Journal of Nondestructive Testing \& Ultrasonics, 6(2).

20. Bar-Cohen, Y., and Crane, R., 1982, "Acoustic-Backscattering Imaging of Subcritical Flaws in Composites," Materials Evaluation, 40, pp. 970-975.

21. Raju, B. B., 1986, “Acoustic-Backscattering Studies of Transverse Cracks in Composite Thick Laminates,” Experimental Mechanics, 26, pp. 71-78.

22. Gorman, M., 1991, “Ultrasonic Polar Backscatter Imaging of Transverse Matrix Cracks," Journal of Composite Materials, 25, pp. 1499-1514. 
23. Steiner, K. V., Eduljee, R. F., Huang, X., and Gillespie, J. W., 1995, “Ultrasonic NDE Techniques for the Evaluation of Matrix Cracking in Composite Laminates,” Composites Science and Technology, 53, pp. 193-198.

24. Aymerich, F., and Meili, S., 2000, "Ultrasonic Evaluation of Matrix Damage in Impacted Composite Laminates," Composites: Part B, 31, pp. 1-6.

25. SONIX Inc., C-scan product line. Website (Last Accessed: $23^{\text {rd }}$ September 2004): http://www.sonix.com/products/c-scan.php3

26. Ultrasonic Transducer Technical Notes, Ultrasonic Transducer Catalogue, Panametrics. Website (Last Accessed: $23^{\text {rd }}$ September 2004): http://www.panametrics-ndt.com/ndt/ndt_transducers/immersion/index.html

27. Kino, G. S., 1987, Acoustic Waves: Devices, Imaging and Analog Signal Processing, Prentice-Hall, Englewood Cliffs, New Jersey.

28. Kline, R. A., 1992, Nondestructive Characterization of Composite Media, Technomic Publishing Company, Lancaster, Pennsylvania.

29. Nayfeh, A. H., 1995, Wave Propagation in Layered Anisotropic Media, Elsevier Science B.V., Amsterdam, The Netherlands.

30. Liu, G. R., and Xi, Z. C., 2002, Elastic Waves in Anisotropic Laminates, CRC Press LLC, Boca Raton, Florida.

31. Jamison, R., Schulte, K., Reifsnider, K., and Stinchcomb, W., 1984, "Characterization and Analysis of Damage Mechanisms in Tension-tension Fatigue of Graphite/epoxy Laminates," in Effects of Defects in Composite 
Materials, American Society for Testing and Materials, ASTM STP 836, pp. 2155.

32. Highsmith, A. L., Stinchcomb, W. W., and Reifsnider, K. L., 1984, "Effect of Fatigue-induced Defects on Residual Response of Composite Laminates," in Effects of Defects in Composite Materials, American Society for Testing and Materials, ASTM STP 836, pp. 194-216.

33. Lavoie, A., and Adolfsson, E., 2001, "Stitch Cracks in Constraint Plies Adjacent to a Cracked Ply,” Journal of Composite Materials, 35, pp. 2077-2097. 


\section{APPENDIX}

\section{WAVE PROPAGATION IN COMPOSITES}

The anisotropic nature of fiber-reinforced composites considerably increases the degree of difficulty in the analysis of wave propagation due to the directional dependence of elastic properties. In this chapter, various concepts in wave propagation, namely, velocity surfaces, slowness surfaces and the application of Snell's law are derived from a variety of sources [28-30] and presented as an easy-to-understand cohesive whole.

\section{A.1.1 Governing Equations}

In the absence of body forces, the equations of motion for a continuous medium may be written as

$$
\sigma_{i j, j}=\rho \ddot{u}_{i}
$$

Where $\sigma_{i j}$ is the stress tensor, $\rho$ is the material density, $u_{i}$ is the displacement vector,

$$
\left.(),{ }_{j}=\frac{\partial}{\partial x_{j}} \text { and } \ddot{(}\right)=\frac{\partial^{2}}{\partial t^{2}}
$$

The constitutive equation for a linearly elastic material is given by

$$
\sigma_{i j}=C_{i j k l} \varepsilon_{k l}
$$

Where $C_{i j k l}$ is the stiffness tensor and $\varepsilon_{k l}$ is the strain tensor.

It follows that, 


$$
\begin{gathered}
\sigma_{i j, j}=C_{i j k l} \varepsilon_{k l, j} \\
C_{i j k l} \varepsilon_{k l, j}=\rho \ddot{u}_{i}
\end{gathered}
$$

Next, the strain-displacement relation, for small displacements, is given by

$$
\varepsilon_{k l}=\frac{1}{2}\left(u_{k, l}+u_{l, k}\right)
$$

Using this relation, equation (A.2) can be written as

$$
\frac{1}{2}\left(C_{i j k l} u_{k, l j}+C_{i j k l} u_{l, k j}\right)=\rho \ddot{u}_{i}
$$

Considering the symmetry of stress and strain tensors, it can be readily shown that

$$
C_{i j k l} u_{k, l j}=\rho \ddot{u}_{i}
$$

For harmonic bulk wave propagation, the solutions for the displacement field given by the above equation may be expressed as

$$
u_{i}=U p_{i} e^{\mathrm{i}\left(k\left(n_{i} x_{i}\right)-\omega t\right)}
$$

where

$U=$ Displacement amplitude

$\boldsymbol{p}=$ Polarization vector

$k=$ Wave number

$\boldsymbol{n}=$ Direction of propagation

$\omega=$ Frequency

Using equation (A.4), we have the following relation for $u_{k, l j}$

$$
u_{k, l j}=-U k^{2} n_{l} n_{j} \alpha_{k} e^{\mathrm{i}\left(k\left(n_{k} x_{k}\right)-\omega t\right)}
$$


Replacing subscript $i$ by $k$ in equation (A.4), and differentiating twice with respect to the time, $\ddot{u}_{k}$ may be written as

$$
\ddot{u}_{k}=-U \omega^{2} p_{k} e^{\mathrm{i}\left(\boldsymbol{k}\left(n_{k} x_{k}\right)-\omega t\right)}
$$

From which the expression for $\ddot{u}_{i}$ follows as

$$
\ddot{u}_{i}=\ddot{u}_{k} \delta_{i k}=-U \omega^{2} p_{k} e^{\mathrm{i}\left(\boldsymbol{k}\left(n_{k} x_{k}\right)-\omega t\right)} \delta_{i k}
$$

Substitution of equations (A.5) and (A.7) in equation (A.3) results in the following expression

$$
\begin{array}{r}
\left(C_{i j k l} n_{j} n_{l}-\rho \frac{\omega^{2}}{k^{2}} \delta_{i k}\right) p_{k}=0 \\
\left(C_{i j k l} n_{j} n_{l}-\rho v^{2} \delta_{i k}\right) p_{k}=0
\end{array}
$$

where $v=\omega / k$ is the phase velocity. Introducing a second order tensor $A_{i k}=C_{i j k l} n_{j} n_{l}$, we have

$$
\left(A_{i k}-\rho v^{2} \delta_{i k}\right) p_{k}=0
$$

Equations represented by (A.9) are the well-known Christoffel equations and represents an eigenvalue problem, where the eigenvalues $\left(\rho v^{2}\right)$ yield the phase velocities $v$ in a given direction $(\boldsymbol{n})$ and the eigenvectors $p_{k}$ are the corresponding particle displacement or polarization vectors. In general, three possible values of $v$ accompanied by corresponding polarization vectors are possible, representing three possible propagating waves. It is clear that $A_{i k}$ being symmetric with real elements will yield real eigenvalues and corresponding orthogonal eigenvectors. In the expanded matrix form, Christoffel equation may be written as follows: 


$$
\left(\begin{array}{ccc}
A_{11}-\rho v^{2} & A_{12} & A_{13} \\
A_{21} & A_{22}-\rho v^{2} & A_{23} \\
A_{31} & A_{32} & A_{33}-\rho v^{2}
\end{array}\right)\left(\begin{array}{l}
p_{1} \\
p_{2} \\
p_{3}
\end{array}\right)=0
$$

Since $A_{i k}=C_{i j k l} n_{j} n_{l}$, the elements of $A_{i k}$ are given by the following expressions, where the reduced notation (Voigt notation : $C_{\alpha \beta}$ ) of $C_{i j k l}$ has been used.

$$
\left.\begin{array}{l}
A_{11}=C_{11} n_{1}^{2}+C_{66} n_{2}^{2}+C_{55} n_{3}^{2}+2 C_{16} n_{1} n_{2}+2 C_{15} n_{1} n_{3}+2 C_{56} n_{2} n_{3} \\
A_{12}=A_{21}=C_{16} n_{1}^{2}+C_{26} n_{2}^{2}+C_{45} n_{3}^{2}+\left(C_{12}+C_{66}\right) n_{1} n_{2}+\left(C_{14}+C_{56}\right) n_{1} n_{3}+\left(C_{46}+C_{25}\right) n_{2} n_{3} \\
A_{13}=A_{31}=C_{15} n_{1}^{2}+C_{46} n_{2}^{2}+C_{35} n_{3}^{2}+\left(C_{14}+C_{56}\right) n_{1} n_{2}+\left(C_{13}+C_{55}\right) n_{1} n_{3}+\left(C_{36}+C_{45}\right) n_{2} n_{3} \\
A_{22}=C_{66} n_{1}^{2}+C_{22} n_{2}^{2}+C_{44} n_{3}^{2}+2 C_{26} n_{1} n_{2}+2 C_{46} n_{1} n_{3}+2 C_{24} n_{2} n_{3} \\
A_{23}=A_{32}=C_{56} n_{1}^{2}+C_{24} n_{2}^{2}+C_{34} n_{3}^{2}+\left(C_{46}+C_{25}\right) n_{1} n_{2}+\left(C_{36}+C_{45}\right) n_{1} n_{3}+\left(C_{23}+C_{44}\right) n_{2} n_{3} \\
A_{33}=C_{55} n_{1}^{2}+C_{44} n_{2}^{2}+C_{33} n_{3}^{2}+2 C_{45} n_{1} n_{2}+2 C_{35} n_{1} n_{3}+2 C_{34} n_{2} n_{3}
\end{array}\right\}
$$

Thus, with the knowledge of the components of stiffness matrix $C_{i j k l}\left(C_{\alpha \beta}\right.$ in the reduced notation) for a material, phase velocities may be easily found by determining the eigenvalues of Christoffel equation i.e., by setting

$$
\left|A_{i k}-\rho v^{2} \delta_{i k}\right|=0
$$

Depending on the angle between the polarization vectors $(\boldsymbol{p})$ and the direction of propagation $(\boldsymbol{n})$, the three waves are termed as quasi-longitudinal (P wave), quasi-shear horizontal (SH wave) and quasi-shear vertical (SV wave). Since, bulk of the discussion presented in this thesis will be concerned with waves propagating from one medium to another, it is convenient to define the $\mathrm{SH}$ and $\mathrm{SV}$ waves with respect to the boundary between the two media. Accordingly, SH wave will be defined as the one in which polarization is parallel to the plane containing the boundary and the SV wave will be 
defined as the one in which the polarization is at an oblique angle to the plane containing the boundary.

\section{A.1.2 The Concept of Slowness Surface}

For a given material, the Christoffel equation may be solved for three phase velocity vectors, as described in the preceding paragraph. Associated with each velocity $(v)$, we may define slowness $(s)$ as $s=\frac{1}{v}$, and the slowness vector as

$$
s=\frac{1}{v}(\boldsymbol{n})
$$

For each wave, the end points of the corresponding slowness vectors generate a locus of points called as the slowness surface or slowness sheet.

For an isotropic material, for each wave, the phase velocities are independent of the direction of propagation giving rise to spherical slowness surfaces. Two eigenvectors degenerate to one, and any direction normal to $\boldsymbol{n}$ becomes an admissible eigenvector. As an example, the 3-dimensional slowness surfaces were calculated for aluminum and are illustrated in Fig. A-1(a). In an effort to reveal the nature of these surfaces, only half of the spherical surfaces are shown. The inner surface corresponds to the $\mathrm{P}$ wave and the outer surface corresponds to both the shear ( $\mathrm{SV}$ and $\mathrm{SH}$ ) waves. Intersection of the velocity surface with the $X_{1}-X_{2}$ plane is shown in Fig. A-1(b). The stiffness matrix for aluminum used in the calculations is shown in equation (A.14) [29]. 


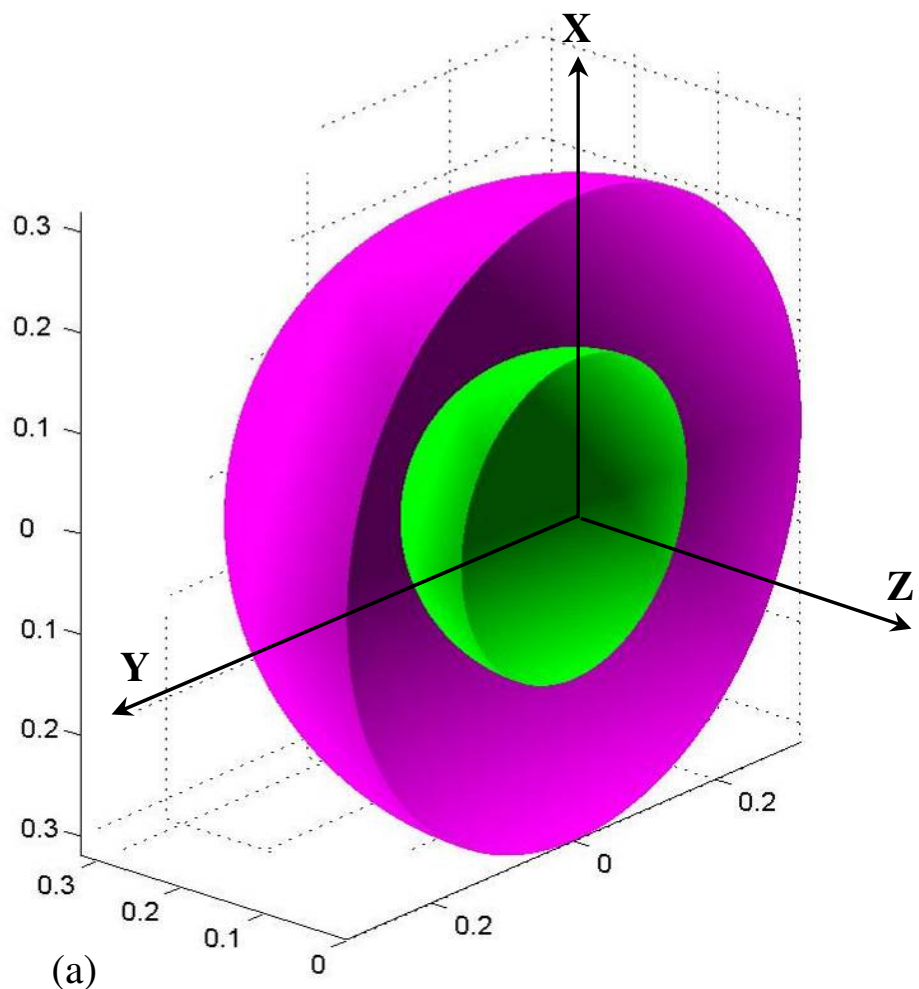

(a)

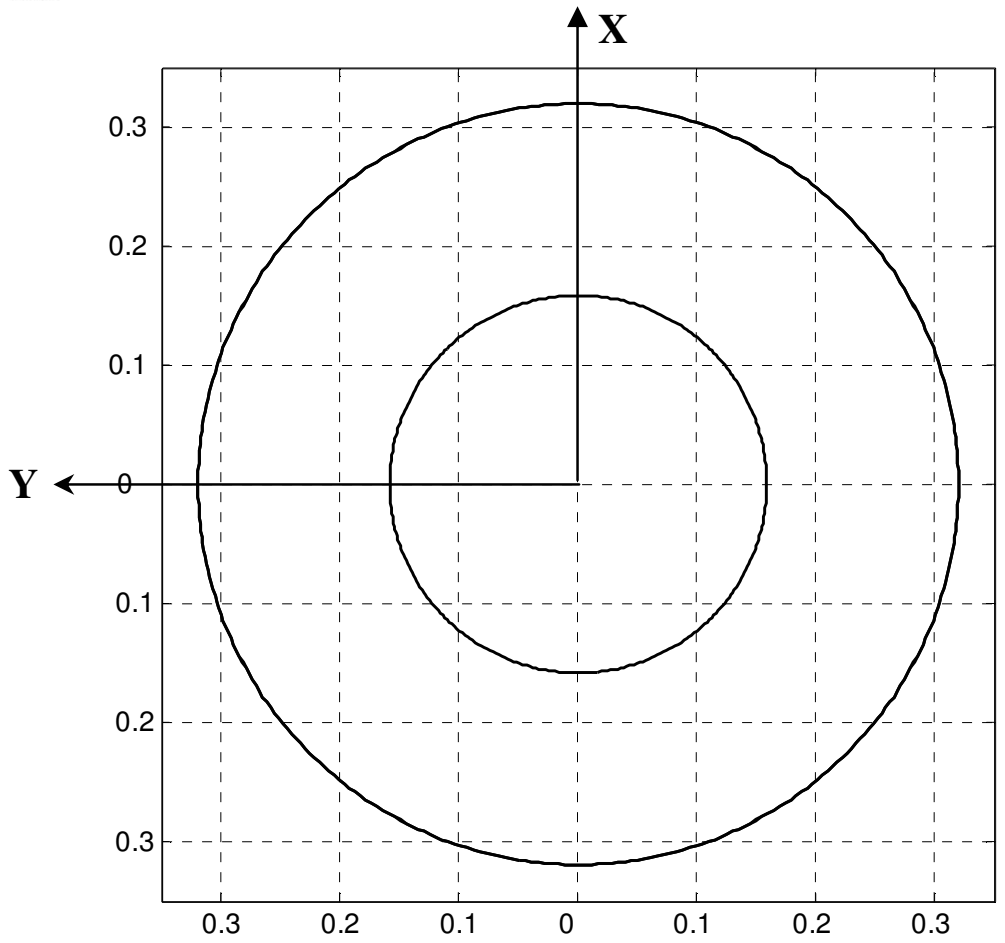

(b)

Fig. A-1 Slowness surface for aluminum. (a) 3-dimensional view, (b) Intersection of the surface with the $X_{1}-X_{2}$ plane. The inner surface corresponds to the longitudinal wave and the outer surface corresponds to the shear waves. Values are in s/mm. 


$$
C_{\alpha \beta}^{\text {aluminum }}=\left[\begin{array}{cccccc}
107.5 & 54.59 & 54.59 & 0 & 0 & 0 \\
54.59 & 107.5 & 54.59 & 0 & 0 & 0 \\
54.59 & 54.59 & 107.5 & 0 & 0 & 0 \\
0 & 0 & 0 & 26.45 & 0 & 0 \\
0 & 0 & 0 & 0 & 26.45 & 0 \\
0 & 0 & 0 & 0 & 0 & 26.45
\end{array}\right] \mathrm{GPa}
$$

For an anisotropic medium, unlike in an isotropic medium, there will be three distinct slowness surfaces corresponding to the three waves. Moreover, the shape of the slowness surface will depend on the directional variation of material properties. The nature of wave propagation in anisotropic media is essentially dictated by the shape of the slowness surfaces.

\section{A.1.3 Computing Slowness Surfaces for Composites}

Laminated composites are made by stacking together individual unidirectional fiber-reinforced plies. Each ply consists of fibers oriented in a particular direction. Two orthogonal co-ordinate systems are chosen in such a way that the $\mathrm{X}-\mathrm{Y}-\mathrm{Z}$ define the laminate (global) coordinates and $\mathrm{x}_{1}-\mathrm{x}_{2}-\mathrm{x}_{3}$ define the material (local) co-ordinates at the ply level. This is shown in Fig. A-2. In the material co-ordinate system, the fibers are generally chosen to be along the direction of the $\mathrm{x}_{1}$-axis. The Z-axis and the $\mathrm{x}_{3}$-axis are perpendicular to the plane of the laminate. The angle $\theta$ defines the rotation of the material co-ordinate system about the Z-axis i.e. $\theta$ is the orientation of fibers in a given ply. 


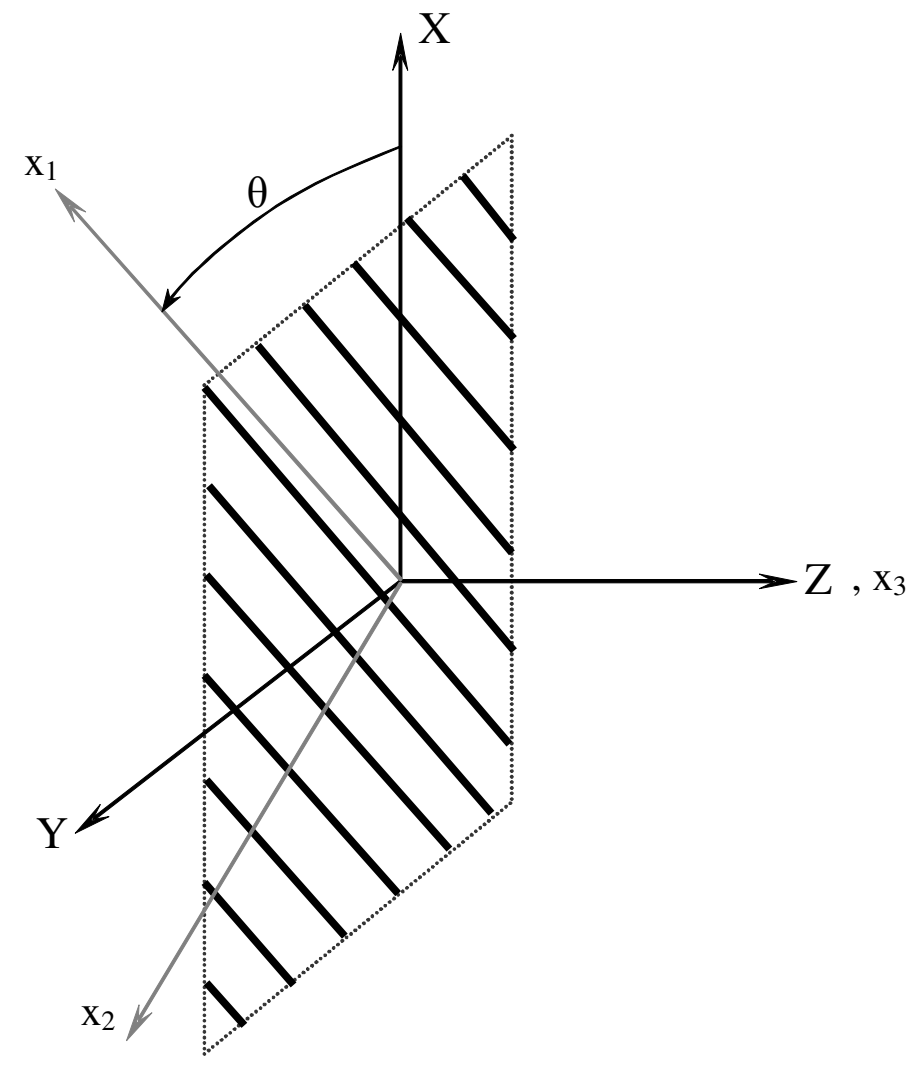

Fig. A-2 Definition of local $\left(\mathrm{x}_{1}-\mathrm{x}_{2}-\mathrm{X}_{3}\right)$ and global (X-Y-Z) coordinate systems with respect to a unidirectional fiber-reinforced lamina. 
In the material co-ordinate system, an individual ply can be treated as a transversely isotropic material, such that, $\mathrm{x}_{1}$-axis is the axis of symmetry and $\mathrm{x}_{2}-\mathrm{x}_{3}$ plane is the plane of transverse isotropy. Five independent elastic constants are required to characterize such materials. These are: Young's moduli along the fiber direction and in the transverse direction, $E_{11}$ and $E_{22}$ respectively; out-of-plane shear modulus $G_{12}$; out-of-plane Poisson's ratio $v_{12}$; and in-plane Poisson's ratio $v_{23}$. Using these engineering constants, the elements of the stiffness matrix $C_{\alpha \beta}$, in the material co-ordinates, can be calculated. Then, the transformed stiffness in the global co-ordinates $\left(\tilde{C}_{\alpha \beta}\right)$ can be determined by

$$
\tilde{C}_{\alpha \beta}=[T]\left[C_{\alpha \beta}\right][T]^{T}
$$

where, the transformation matrix $T$ given by

$$
[T]=\left[\begin{array}{cccccc}
\cos ^{2} \theta & \sin ^{2} \theta & 0 & 0 & 0 & -\sin 2 \theta \\
\sin ^{2} \theta & \cos ^{2} \theta & 0 & 0 & 0 & \sin 2 \theta \\
0 & 0 & 1 & 0 & 0 & 0 \\
0 & 0 & 0 & \cos \theta & \sin \theta & 0 \\
0 & 0 & 0 & -\sin \theta & \cos \theta & 0 \\
\sin \theta \cos \theta & -\sin \theta \cos \theta & 0 & 0 & 0 & \cos ^{2} \theta-\sin ^{2} \theta
\end{array}\right]
$$

Next, consider the direction of wave propagation vector $(\boldsymbol{n})$ to be oriented in an arbitrary direction in space such a way that it makes an angle $\phi$ with the Y-Z plane and its projection on the $\mathrm{X}-\mathrm{Z}$ plane makes an angle $\psi$ with the $\mathrm{Z}$ axis so that

$$
\boldsymbol{n}=\left(\begin{array}{c}
\sin \phi \\
\cos \phi \sin \psi \\
\cos \phi \cos \psi
\end{array}\right)
$$


Now, using this $n$ and the transformed stiffness matrix $\tilde{C}_{\alpha \beta}$, as given by the equation (A.15), the values of $A_{i k}$ can be found according to the equation (A.11). Then the phase velocities are easily found by solving the eigenvalue formulation represented in equation (A.12). At each orientation of $\boldsymbol{n}$ in space, the solution will yield three phase velocities and three corresponding values for slowness vectors as given by the equation (A.13). If the propagation vector $\boldsymbol{n}$ is rotated over all possible values of $\phi$ and $\psi$, a slowness surface is obtained as given by the locus of the endpoints of the slowness vectors.

Following the procedure described above, slowness surfaces were obtained for a unidirectional graphite/epoxy composites with fibers oriented at three different angles: $0^{\circ}, 45^{\circ}$ and $90^{\circ}$. The elastic properties of the IM7/977-2 graphite/epoxy system at 0.6 fiber volume fraction (listed in Table A-1) were used for the calculations. The elements of the stiffness matrix $C_{\alpha \beta}$ are shown in equation (A.17).

$C_{\alpha \beta}=\left[\begin{array}{cccccc}161.20 & 4.39 & 4.39 & 0 & 0 & 0 \\ 4.39 & 12.03 & 5.55 & 0 & 0 & 0 \\ 4.39 & 5.55 & 12.03 & 0 & 0 & 0 \\ 0 & 0 & 0 & 2.57 & 0 & 0 \\ 0 & 0 & 0 & 0 & 4.34 & 0 \\ 0 & 0 & 0 & 0 & 0 & 4.34\end{array}\right] \mathrm{GPa}$

The slowness surfaces for the $0^{\circ}, 45^{\circ}$ and $90^{\circ}$ plies are illustrated in Fig. A-3, Fig. A-4 and Fig. A-5 respectively. For the sake of clarity and ease of interpretation, only a half of the slowness surfaces are displayed. For the purpose of analysis, a 2-dimensional 
Table A-1 Elastic constants for a IM7/977-2 unidirectional composite ( 0.6 fiber volume fraction).

\begin{tabular}{|l|l|}
\hline $\mathrm{E}_{11}$ & $159 \mathrm{GPa}$ \\
\hline $\mathrm{E}_{22}$ & $9.43 \mathrm{GPa}$ \\
\hline $\mathrm{G}_{12}$ & $4.34 \mathrm{GPa}$ \\
\hline$v_{12}$ & 0.253 \\
\hline$v_{23}$ & 0.456 \\
\hline
\end{tabular}



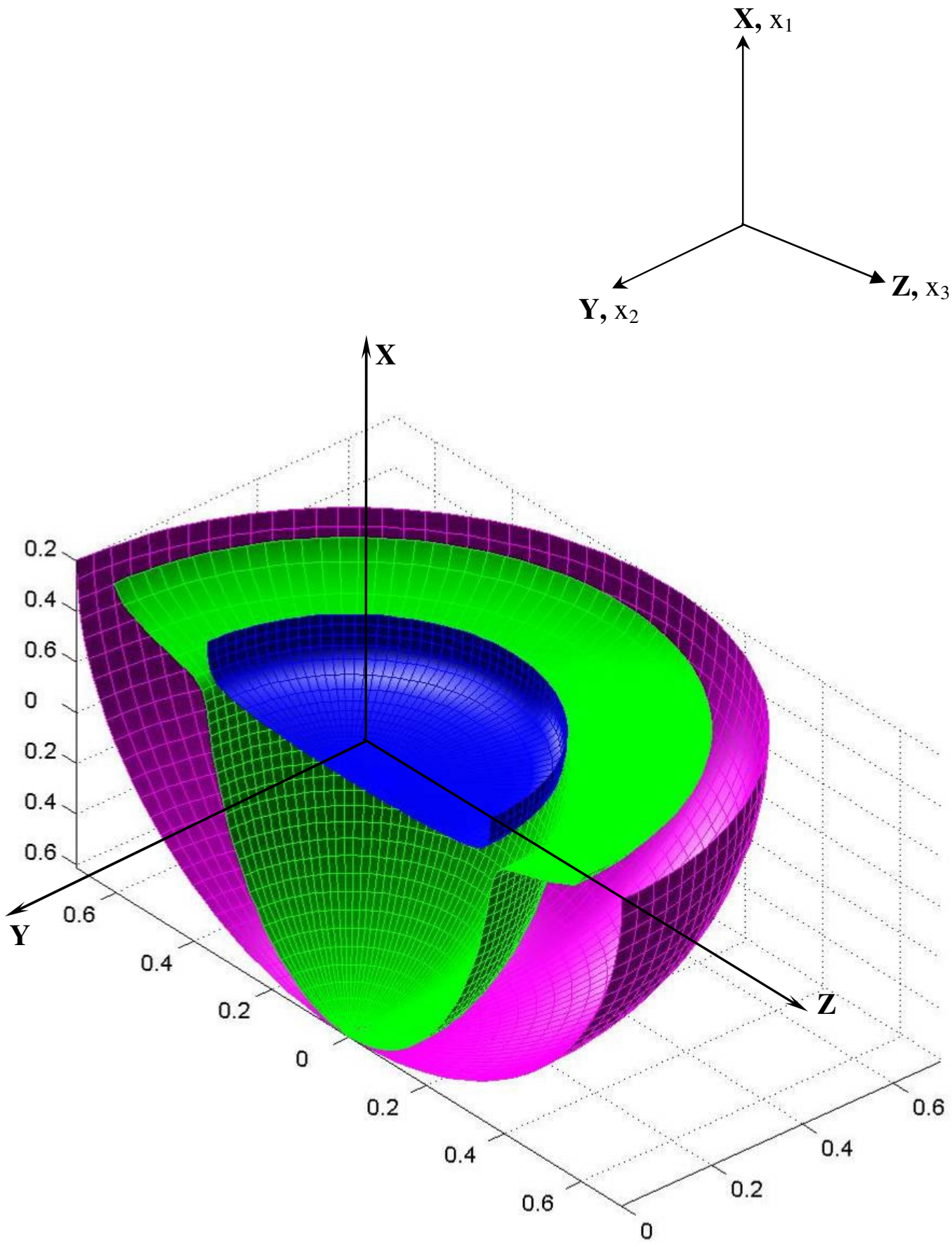

Fig. A-3 Slowness surfaces for a $0^{\circ}$ ply. The innermost surface corresponds to the $\mathrm{P}$-waves, the next surface corresponds to the SV waves and the outermost surface corresponds to the $\mathrm{SH}$ waves. Values are in units of s/mm. 


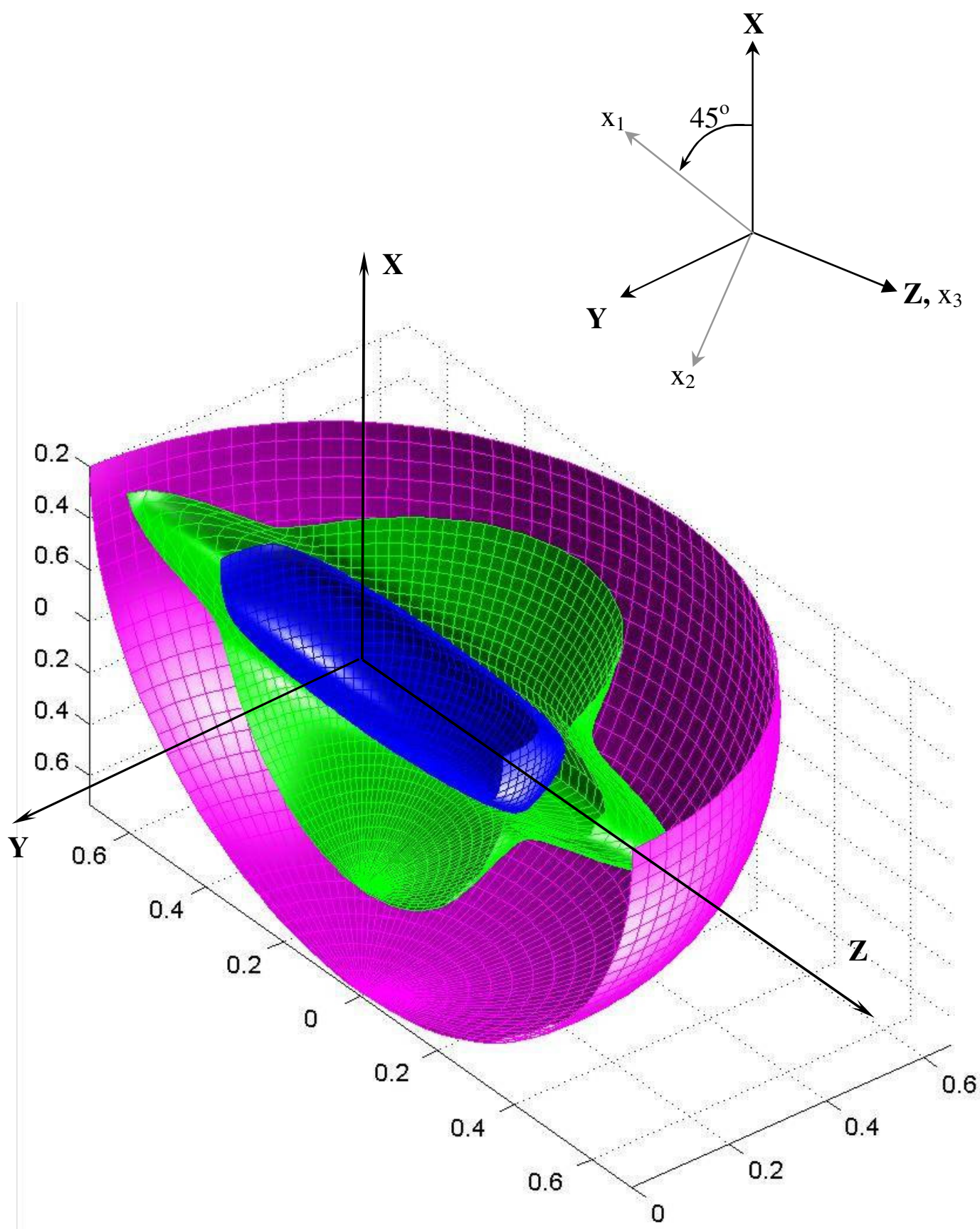

Fig. A-4 Slowness surfaces for a $45^{\circ}$ ply. The innermost surface corresponds to the P-waves, the next surface corresponds to the SV waves and the outermost surface corresponds to the SH waves. Values are in units of s/mm. 


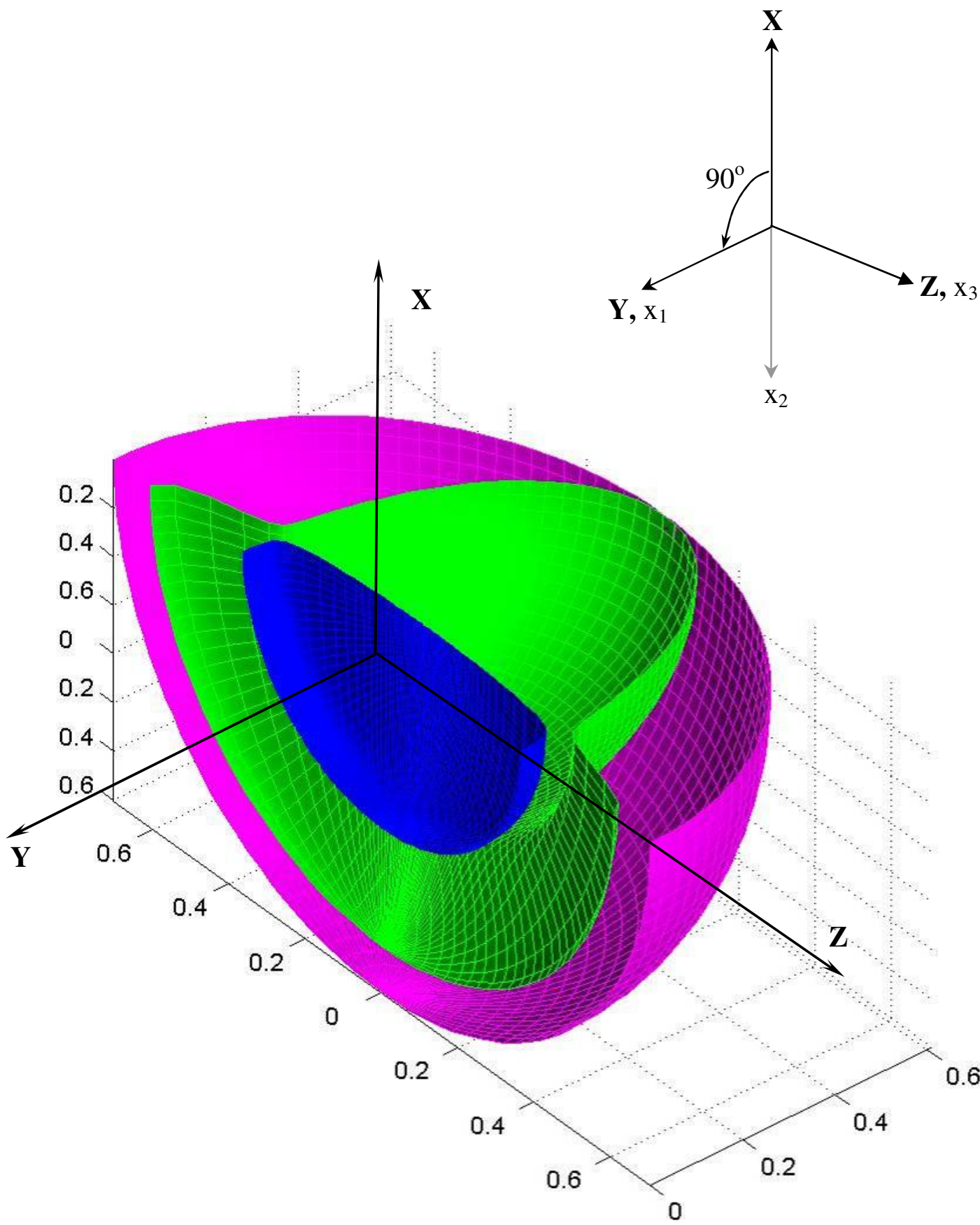

Fig. A-5 Slowness surfaces for a $90^{\circ}$ ply. The innermost surface corresponds to the $\mathrm{P}$-waves, the next surface corresponds to the SV waves and the outermost surface corresponds to the SH waves. Values are in units of s/mm. 


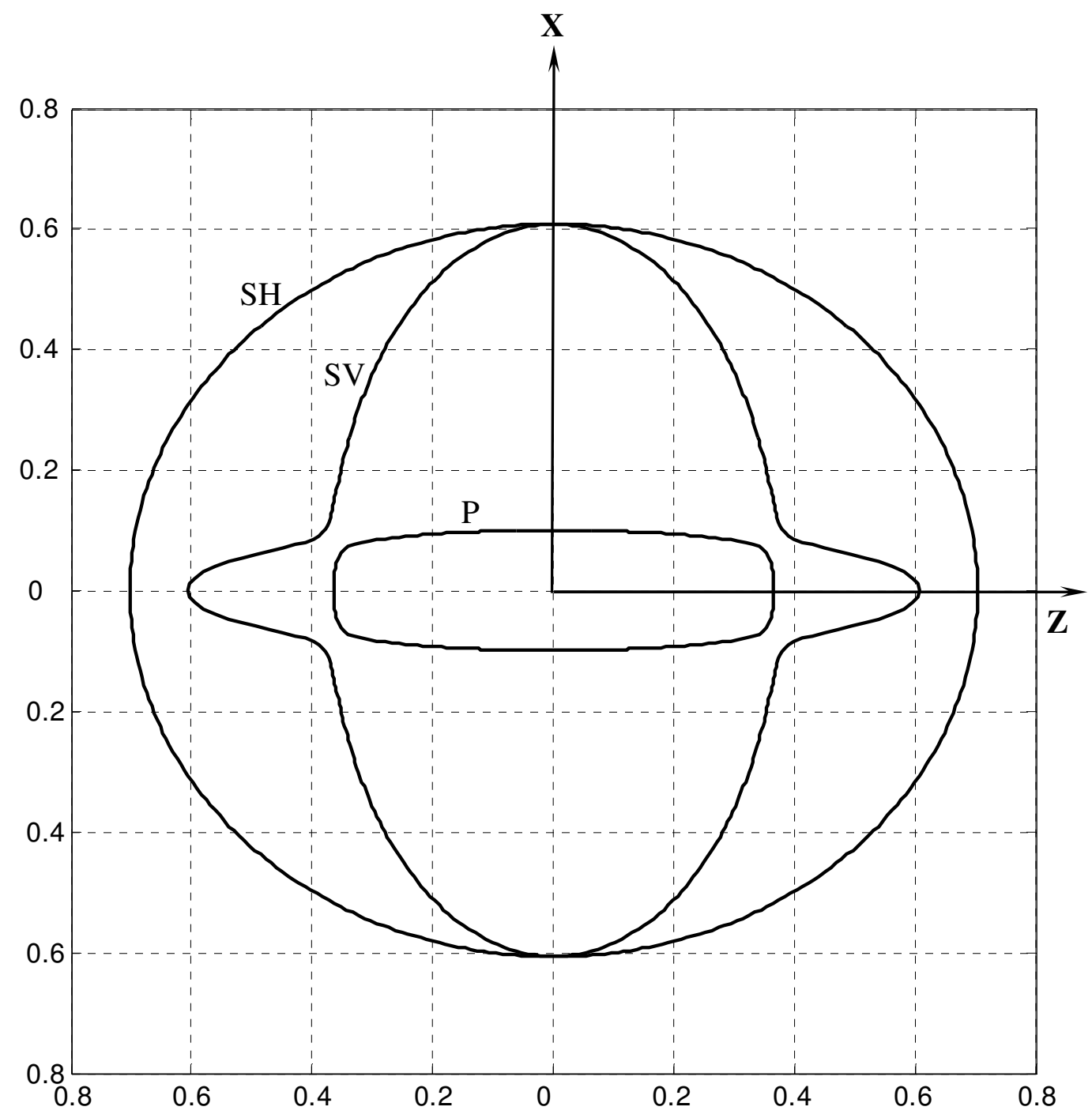

Fig. A-6 Slowness diagram for a $0^{\circ}$ ply as seen on the X-Z plane. Values are in units of $\mathrm{s} / \mathrm{mm}$. 


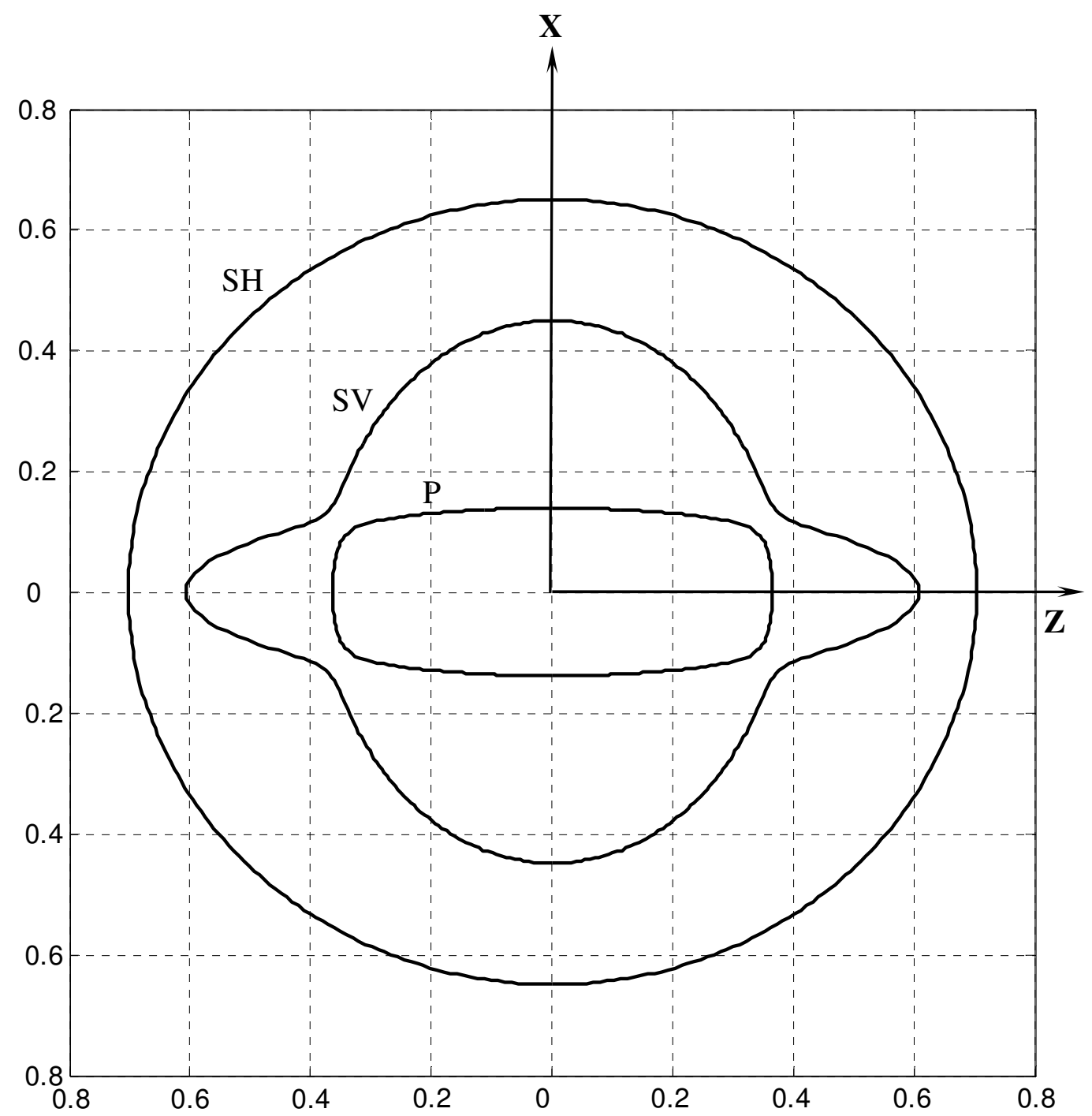

Fig. A-7 Slowness diagram for a $45^{\circ}$ ply as seen on the $\mathrm{X}-\mathrm{Z}$ plane. Values are in units of $\mathrm{s} / \mathrm{mm}$. 


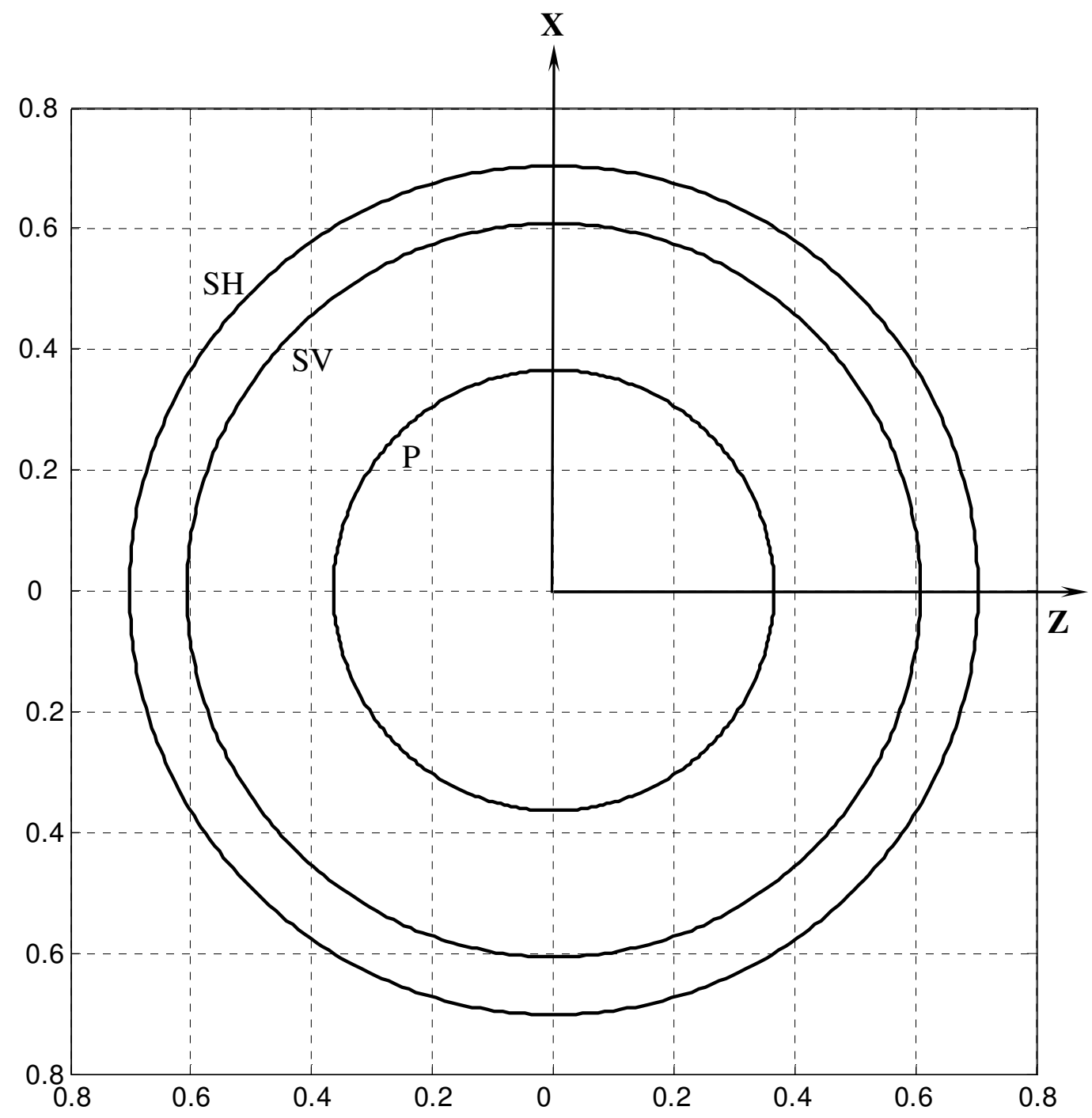

Fig. A-8 Slowness diagram for a $90^{\circ}$ ply as seen on the $\mathrm{X}-\mathrm{Z}$ plane. Values are in units of s/mm. 
slowness profile (called slowness diagram) is generally used. This is done by setting $\psi=0$ in equation (A.16) and rotating $\boldsymbol{n}$ for all values of $\phi$. Fig. A-6, Fig. A-7 and Fig. A-8 show the 2-dimensional slowness profile for plies oriented at $0^{\circ}, 45^{\circ}$ and $90^{\circ}$ respectively.

\section{A.1.4 Snell's Law and Slowness Diagrams}

When a wave propagates from one medium to another, a number of waves are reflected and transmitted propagating in the originating and the continuing medium. For an obliquely incident wave, due to the differences in the elastic properties of the two media, refraction of the transmitted waves takes place. In general, for each incident wave there will three reflected and three transmitted components. In the case where the continuing medium is anisotropic, the number of waves, the angle of refraction and phase velocities are governed by two constraints: 1. Snell's law and 2. Slowness diagrams. This is explained in the following with the help of a specific example of a longitudinal wave originating in water and propagating in a $90^{\circ}$ ply of a composite.

The slowness diagram representation for waves propagating from water to a $0^{\circ}$ ply is shown in Fig. A-9. Water is modeled as an isotropic, non-viscous fluid and hence can support only a P-wave. As a result the slowness diagram for water shows only one circular profile with radius $\left(S_{\mathrm{w}}\right)$ given by

$$
S_{w}=\frac{1}{v_{w}}
$$




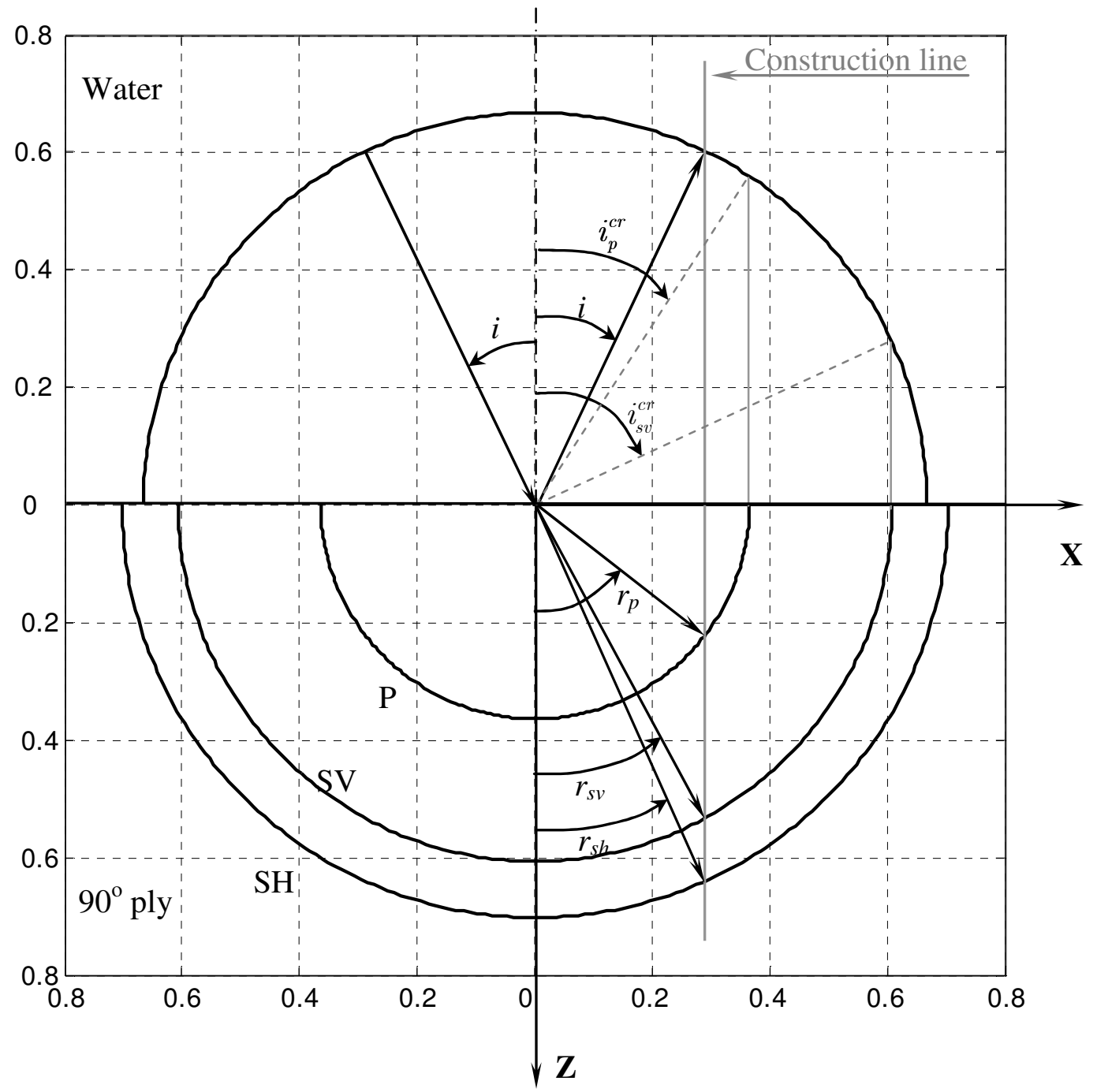

Fig. A-9 Slowness diagram for a water $/ 90^{\circ}$ ply system showing incident, reflected and transmitted waves. Values are in units of s/mm. 
where $v_{\mathrm{w}}$ is the speed of longitudinal waves in water. The slowness diagram for the $90^{\circ}$ ply shows three curves for the $\mathrm{P}, \mathrm{SV}$ and $\mathrm{SH}$ waves respectively. The angles of incidence, reflection and refraction will be measured from the normal to the interface. Suppose the P-wave is incident at an angle $i$ from the vertical, then at the interface, there exists a P-wave reflected at an angle $i$ and three possible transmitted waves in the $0^{\circ}$ ply: $\mathrm{P}, \mathrm{SV}$ and $\mathrm{SH}$ at angles or refraction $r_{p}, r_{s v}$ and $r_{s h}$ respectively. The relation between the angle of incidence and angles of refraction for the three waves is governed by Snell's law as:

$$
\frac{\sin i}{v_{w}}=\frac{\sin r_{p}}{v_{p}\left(r_{p}\right)}=\frac{\sin r_{s v}}{v_{s v}\left(r_{s v}\right)}=\frac{\sin r_{s h}}{v_{s h}\left(r_{s h}\right)}
$$

where $v_{p}\left(r_{p}\right), v_{s v}\left(r_{s v}\right)$ and $v_{s h}\left(r_{s h}\right)$ are the velocities of $\mathrm{P}, \mathrm{SV}$ and $\mathrm{SH}$ waves respectively, expressed as functions of their respective angles of refraction.

Equation (A.17) may also be written in the following form

$$
\begin{gathered}
\left(\frac{1}{v_{w}}\right) \sin i=\left(\frac{1}{v_{p}\left(r_{p}\right)}\right) \sin r_{p}=\left(\frac{1}{v_{s v}\left(r_{s v}\right)}\right) \sin r_{s v}=\left(\frac{1}{v_{s h}\left(r_{s h}\right)}\right) \sin r_{s h} \\
\left(S_{w}\right) \sin i=\left(S_{p}\right) \sin r_{p}=\left(S_{s v}\right) \sin r_{s v}=\left(S_{s h}\right) \sin r_{s h}
\end{gathered}
$$

where $S_{p}, S_{s v}$ and $S_{s h}$ are the slowness of the $\mathrm{P}, \mathrm{SV}$ and the $\mathrm{SH}$ waves at the corresponding angles of refraction $r_{p}, r_{s v}$ and $r_{s h}$ respectively. From, equation (A.20) it is observed that the projections of the slowness of the incident P-wave and all the three transmitted waves on the interface are equal. Consequently, if the speed of P-wave in water is known, then at any angle of incidence, if we draw a guide line at a distance $d$ from the normal such that $d=\left(S_{w}\right) \sin i$, the velocities and the corresponding angles of 
refraction in the $0^{\circ}$ ply may be easily measured at the point of intersection of this line with the slowness curves of the $0^{\circ} \mathrm{ply}$.

An interesting situation arises when the construction line does not intersect one or more of the slowness curves. This happens when the angle of incidence equals or exceeds the critical angle of incidence for the corresponding waves i.e. when the angle of refraction is equal to $90^{\circ}$. Mathematically, the critical angles of incidence $\left(i_{k}^{c r}\right)$ can be obtained from Snell's law as

$$
\begin{aligned}
& \frac{\sin i_{k}^{c r}}{v_{w}}=\frac{\sin (\pi / 2)}{v_{k}} \\
& i_{k}^{c r}=\sin ^{-1}\left(\frac{v_{w}}{v_{k}}\right)
\end{aligned}
$$

where the subscript $k$ stands for either $p, s v$ or $s h$ as may be the case. On the slowness diagram, this happens when the guide line is tangential to the slowness curve. Beyond the critical angle, in the continuing medium, the waves become evanescent and exponentially decay with distance from the interface along the normal. A rigorous consideration of wave propagation in the presence of evanescent waves is beyond the scope of this thesis.

Similar analysis was conducted for a P-wave originating in water and incident on $45^{\circ}$ and $90^{\circ}$ plies. The slowness diagrams for the $0^{\circ}, 45^{\circ}$ and $90^{\circ}$ when the angle of incidence is $i=25^{\circ}$ are shown in Fig. A-10, Fig A-11 and Fig. A-12 respectively. Important results from the analyses are listed in Table A-2. It is clear that when a P-wave from water is incident on a $0^{\circ}$ ply or a $45^{\circ}$ ply at $25^{\circ}$ angle of incidence, the refracted P- 
wave becomes evanescent (angle of refraction $=90^{\circ}$ ). This situation occurs in almost all of the specimens tested for this thesis. The presence of evanescent waves complicates the analysis even further and calls for an extremely rigorous, mathematical approach for the wave propagation analysis. Such an analysis, at present, is beyond the scope of this thesis. Future efforts will be directed towards the analytical treatment of the experimental work discussed in this thesis. 

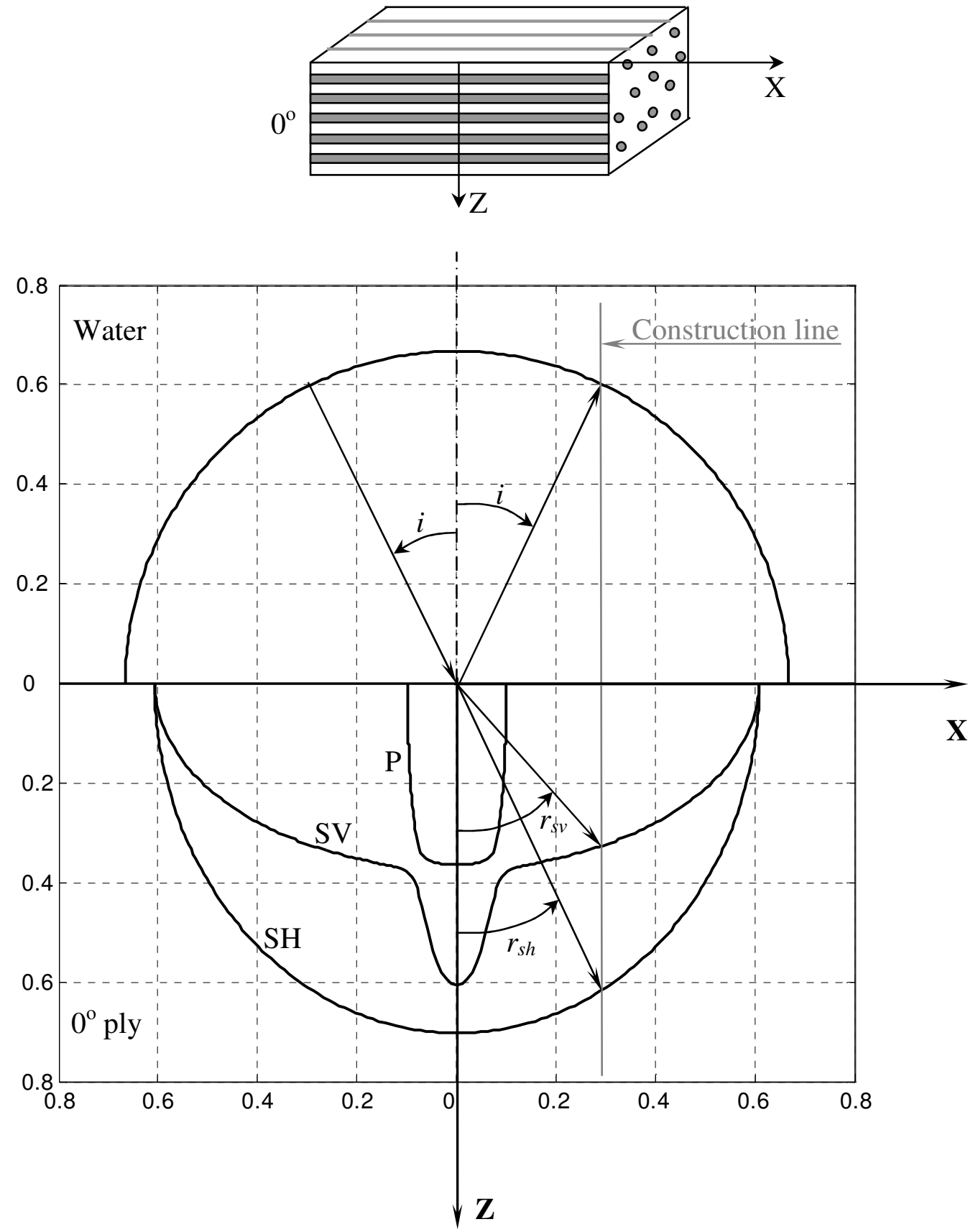

Fig. A-10 Slowness diagram for a water $/ 0^{\circ}$ ply system showing incident, reflected and transmitted waves at $25^{\circ}$ angle of incidence. Values are in units of s/mm. 

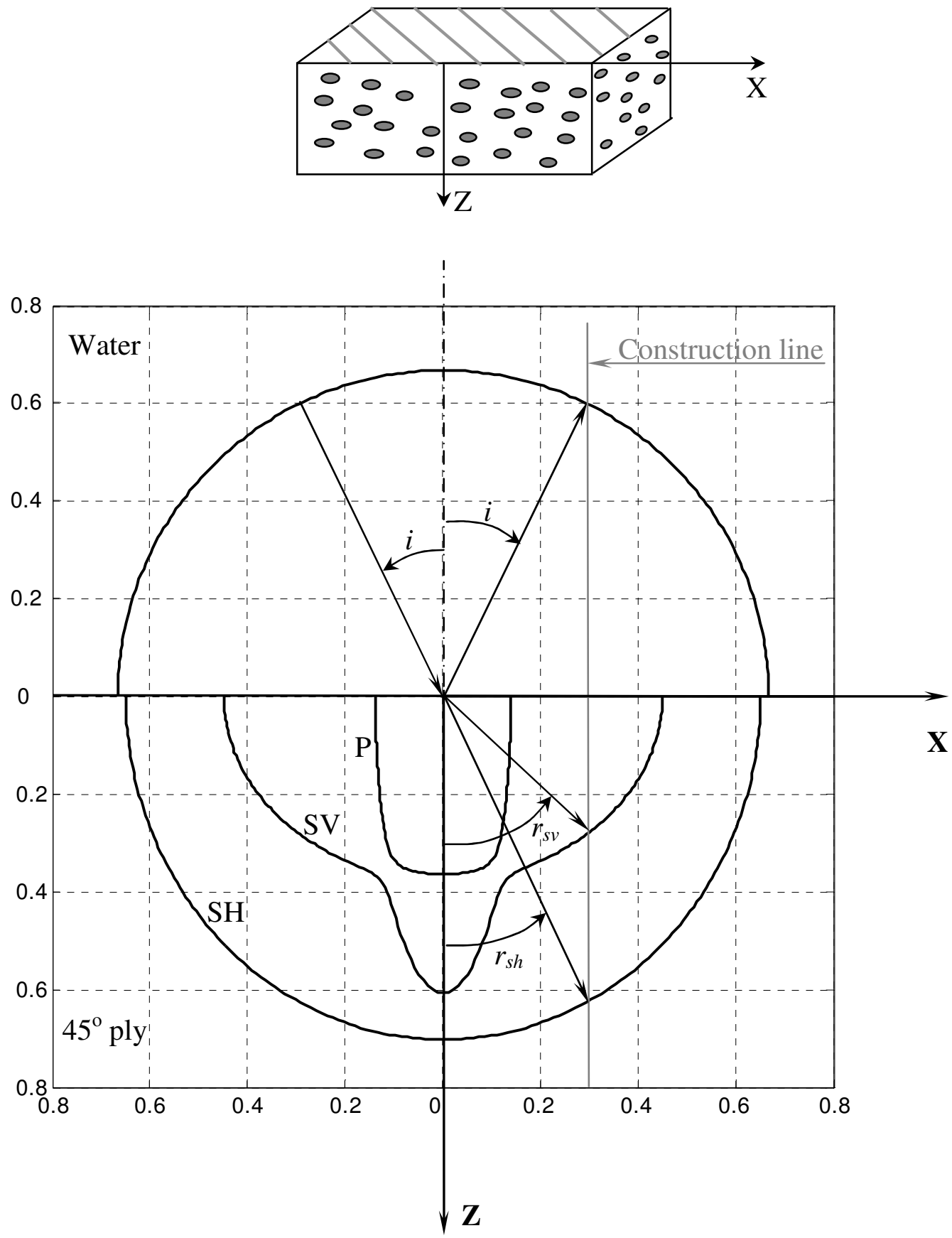

Fig. A-11 Slowness diagram for a water $/ 45^{\circ}$ ply system showing incident, reflected and transmitted waves at $25^{\circ}$ angle of incidence. Values are in units of s $/ \mathrm{mm}$. 

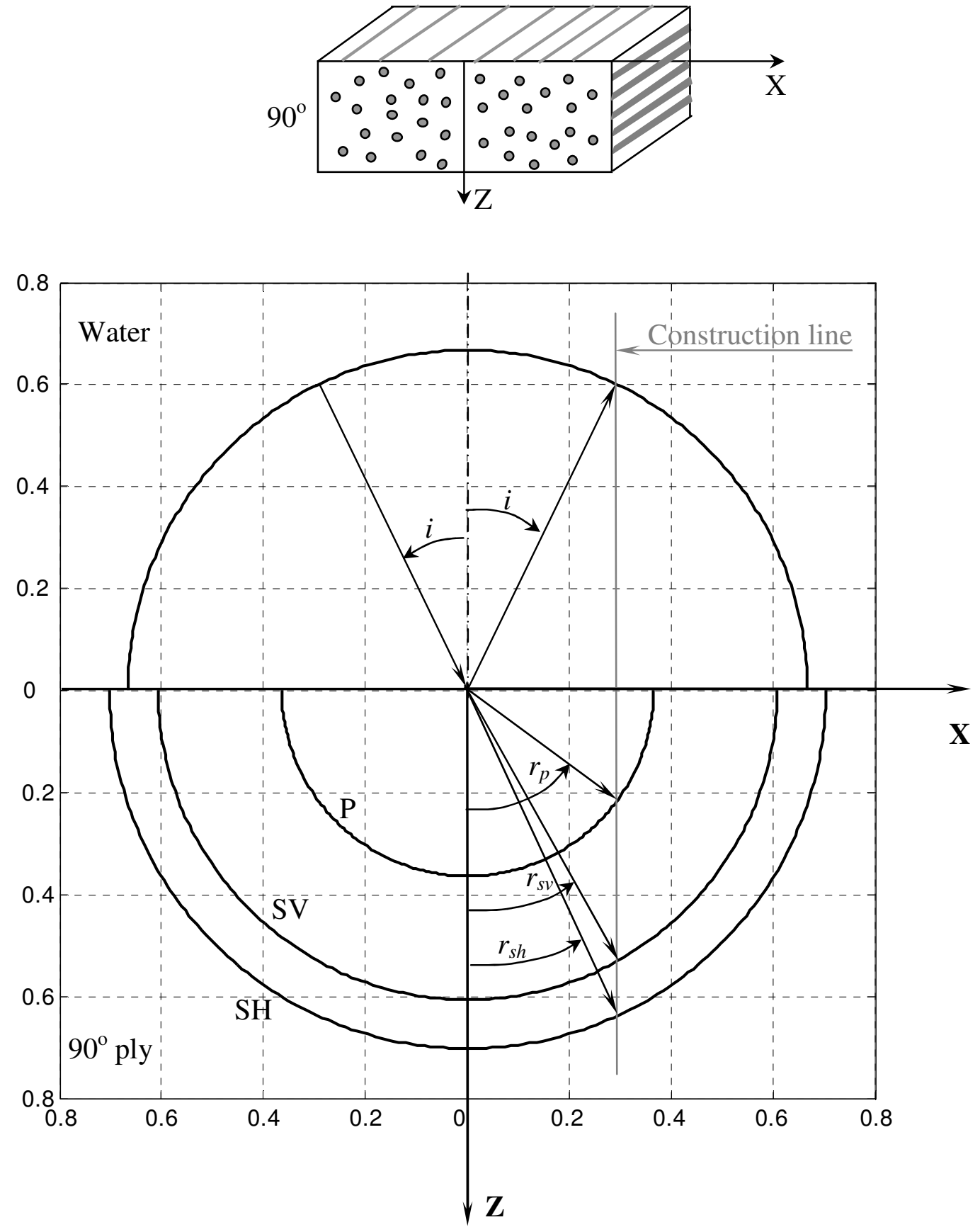

Fig. A-12 Slowness diagram for a water $/ 90^{\circ}$ ply system showing incident, reflected and transmitted waves at $25^{\circ}$ angle of incidence. Values are in units of s/mm. 
Table A-2 Properties of the refracted waves.

(Incident $\mathrm{P}$-wave through water at angle of incidence $i=25^{\circ}$ )

\begin{tabular}{|l|l|l|l|}
\hline & $0^{\circ}$-ply & $45^{\circ}$-ply & $90^{\circ}$-ply \\
\hline$v_{\mathrm{p}}(\mathrm{m} / \mathrm{s})$ & Evanescent & Evanescent & 2775.52 \\
\hline$v_{\mathrm{sv}}(\mathrm{m} / \mathrm{s})$ & 2295.67 & 2469.69 & 1621.39 \\
\hline$r_{\mathrm{p}}($ degree $)$ & 90 & 90 & 51 \\
\hline$r_{\mathrm{sv}}($ degree $)$ & 40 & 43.75 & 27 \\
\hline $\begin{array}{l}\lambda_{20 \mathrm{MHz}}(\mathrm{mm}, \text { across } \\
\text { laminate width) }\end{array}$ & $0.088(\mathrm{SV}$ & $0.089(\mathrm{SV}$ & 0.072 (SV mode) \\
\hline $\begin{array}{l}\lambda_{25 \mathrm{MHz}} \text { (across } \\
\text { laminate width) }\end{array}$ & $0.070(\mathrm{SV}$ & 0.071 (SV & 0.058 (SV mode) \\
& mode) & mode) & 0.070 (P mode) \\
\hline
\end{tabular}




\section{VITA}

Atul Shridatta Ganpatye received his B.E. degree in mechanical engineering from the Veermata Jeejabai Technological Institute (VJTI), Mumbai University, Mumbai, in May 2001. He joined the Department of Aerospace Engineering at the Texas A\&M University in Fall 2001 to pursue a Master of Science degree. Upon completion of his Master of Science degree in December 2004, he plans to pursue doctoral studies at Texas A\&M University.

Permanent Address: 403 Vaishnavidham

P. K. Road, Mulund (W)

Mumbai -400080

India 